University of Tennessee Health Science Center

UTHSC Digital Commons

\title{
TDiscovery and Development of Novel Therapeutic Agents for Advanced Melanoma
}

\author{
Zhao Wang \\ University of Tennessee Health Science Center
}

Follow this and additional works at: https://dc.uthsc.edu/dissertations

Part of the Medicinal and Pharmaceutical Chemistry Commons, and the Pharmaceutics and Drug Design Commons

\section{Recommended Citation}

Wang, Zhao , "TDiscovery and Development of Novel Therapeutic Agents for Advanced Melanoma" (2010). Theses and Dissertations (ETD). Paper 281. http://dx.doi.org/10.21007/etd.cghs.2010.0343.

This Dissertation is brought to you for free and open access by the College of Graduate Health Sciences at UTHSC Digital Commons. It has been accepted for inclusion in Theses and Dissertations (ETD) by an authorized administrator of UTHSC Digital Commons. For more information, please contact jwelch30@uthsc.edu. 


\title{
TDiscovery and Development of Novel Therapeutic Agents for Advanced Melanoma
}

\begin{abstract}
Malignant melanoma is the most dangerous form of skin cancer and accounts for about $75 \%$ of skin cancer deaths. Once diagnosed at the metastatic stage, it has a very poor prognosis with a median survival rate of 6 months and a 5 -year survival rate of less than $5 \%$. In addition, melanoma has become an important public health hazard owing to its rising incidence, which has been well documented over the past 50 years. Currently there is no effective way to treat melanoma. It is highly resistant to existing chemotherapy, radiotherapy, and immunotherapy. Over the past 30 years, only two drugs have been approved by the Food and Drug Administration (FDA) for metastatic melanoma: dacarbazine (DTIC) and interleukin-2 (IL-2). But even with these two drugs, fewer than $15 \%$ of patients have a favorable response and fewer than $5 \%$ of patients reach complete remission. On the other hand, the toxicity associated with DTIC and IL-2 is often significant, resulting in serious or life threatening side effects in many patients. In recent years, great efforts have been made in fighting metastatic melanoma. But neither combinations of DTIC with other chemotherapy drugs (e.g., cisplatin, vinblastine, and carmustine) nor adding interferon$\alpha 2 b$ to DTIC have shown a survival advantage over DTIC treatment alone. Extensive clinical trials with a lot of antibodies and vaccines to treat metastatic melanoma have also failed to demonstrate satisfactory efficacy. Therefore, developing more effective drugs for melanoma is urgently needed.
\end{abstract}

We started our efforts in finding new drugs for melanoma by screening a large compound library. The in vitro cytotoxicity data on several melanoma cell lines led us to the discovery of three active structure scaffolds: serine amides, serine amino alcohols, and arylthiazolidine-4-carboxylic acid amides (ATCAAs). Because ATCAAs showed better selectivity between cancer cells and normal fibroblast cells, the chemists in our group focused on this scaffold and performed extensive structure modifications for structureactivity-relationship (SAR) studies. The SAR results were then used to guide further synthesis in an effort to maximize activity and selectivity. Two active compounds identified during the process were sent to the U.S. National Cancer Institute for anticancer screening using 60 human tumor cell lines. Results showed that these two compounds have extensive cytotoxic activity against all nine types of cancer cells with IC 50 values ranging from $120 \mathrm{nM}$ (leukemia, CCRF-CEM cell line) to $11 \mu \mathrm{M}$ (colon cancer, HCC-15 cell line). One compound showed particularly good activity against melanoma cells $\left(\mathrm{IC}_{50}=130 \mathrm{nM} 1 \mathrm{mM}\right.$ against all eight melanoma cell lines). I then evaluated ATCAAs inhibitory effect on melanoma colony formation and in vivo melanoma tumor growth. The in vivo data were very encouraging. One tested compound significantly inhibited melanoma tumor growth at a dose of $10 \mathrm{mg} / \mathrm{kg}$ and showed higher efficacy than did DTIC at a dose of $60 \mathrm{mg} / \mathrm{kg}$. These findings built up a strong basis for the development of novel chemotherapeutic drugs for advanced melanoma.

Furthermore, the chemists in our group also synthesized some new imidazole and imidazoline analogs by focusing on the SAR studies of the central five-member ring. Although the current compounds displayed lower potency when compared with our lead thiazolidine analogs, they may have the distinct advantage of being more stable in vivo with the reduced necessity of chiral separations. Some of these new compounds have activity similar to Sorafenib, an FDA-approved drug that has been tested clinically in melanoma patients.

To further expand our understanding of SARs and to potentially identify new platforms for active compounds, Dr. Li and Dr. Seibel explored a compound library from the University of Cincinnati's Drug Discovery Center. This library contains 342,910 small molecules. Based on the structure of our lead molecule, two ligand-based virtual screening approaches were used: 1) similarity search based on atom connectivity by using Scitegic Pipeline Pilot software and 2) similarity search based on molecular shape 
by using Schrodinger software. Results showed that these two approaches are highly complementary and lead to different active molecular structures. These structures are quite suitable for further structural modification and provide new platforms for our anticancer drug discovery efforts.

Subsequently, further lead structure optimization led to the discovery of substituted methoxylbenzoyl-arylthiazole (SMART) compounds. To improve solubility and to circumvent the metabolic instability brought by the thiazole ring, our team designed and synthesized a new series of analogs: 2-aryl-4-benzoylimidazoles (ABIs). These two classes of compounds showed great in vitro cytotoxicity against melanoma, and the $\mathrm{IC}_{50}$ of the most active compound was below $10 \mathrm{nM}$. They also showed equal potency against multi-drug resistant melanoma cells and the sensitive parent cells, indicating that these compounds can effectively overcome multi-drug resistance, which is a major cause of cancer chemotherapy failure. In vivo testing on C57BL/6 mice bearing B16-F1 melanoma allograft and on double homozygous SCID (severe combined immunodeficiency) hairless outbred (SHO) mice or athymic nude mice bearing A375 human melanoma xenograft showed these two classes of compounds significantly inhibited melanoma tumor growth. Some compounds even showed substantially better activity than did DTIC, the gold standard anti-melanoma drug. Meanwhile, preliminary toxicity studies suggested that mice can tolerate tested compounds well at effective dose levels. No sign of acute toxicity was observed from the experiments. More importantly, I identified the cellular target for ABI and SMART compounds through a series of biotechniques and molecular modeling studies. Strong experimental evidence has shown that these compounds bind to tubulin at the colchicine binding site in the $\alpha / \beta$-tubulin heterodimers to disrupt functional microtubule formation. In the meantime, I also tested the pharmacokinetic properties of some active compounds in mice together with Mr. Chien-ming Li. With their good in vivo anti-melanoma activity and their ability to overcome multi-drug resistance, these new classes of compounds have great potential for melanoma therapy.

\section{Document Type}

Dissertation

\section{Degree Name}

Doctor of Philosophy (PhD)

\section{Program}

Pharmaceutical Sciences

\section{Research Advisor}

Wei Li, Ph.D.

\section{Keywords}

Melanoma, tubulin, inhibitor, multi-drug resistance, imidazole, thiazole

\section{Subject Categories}

Medicinal and Pharmaceutical Chemistry | Medicine and Health Sciences | Pharmaceutics and Drug Design | Pharmacy and Pharmaceutical Sciences

\section{Comments}

Two year embargo expired May 2010 


\title{
DISCOVERY AND DEVELOPMENT OF NOVEL THERAPEUTIC AGENTS
} FOR ADVANCED MELANOMA

\author{
A Dissertation \\ Presented for \\ The Graduate Studies Council \\ The University of Tennessee \\ Health Science Center
}

\begin{abstract}
In Partial Fulfillment
Of the Requirements for the Degree

Doctor of Philosophy

From The University of Tennessee
\end{abstract}

\author{
By \\ Zhao Wang \\ May 2010
}


Portions of Chapter 2 (C) 2007, 2008, and 2009 by Elsvier Ltd.

Chapter 3 and portions of Chapter 4 (C) 2009 by American Chemical Society. All other material (C) 2010 by Zhao Wang. All rights reserved. 


\title{
DEDICATION
}

\author{
To \\ My parents, \\ My wife, Rui Zhu \\ And \\ My daughter, Sophia Z. Wang \\ For \\ Their endless love and continuous support
}




\section{ACKNOWLEDGMENTS}

I could never have completed this daunting task without the help, support, guidance and efforts from a lot of people. First and foremost, I would like to express my deepest gratitude and sincere appreciation to my mentor Dr. Wei Li for providing me with an opportunity to work in his lab and instilling in me the qualities of being a good scientist. His co-operation and continued support have been major driving forces through my graduate career at the University of Tennessee. I would also like to thank my committee members, Drs. Miller, Slominski, Meibohm and Kim for their invaluable suggestions, guidance and assistance over the years. I would like to especially acknowledge Dr. Miller for providing me with the compounds and continuous encouragement and support on the group meetings we held together. I am also very grateful for Dr. Slominski in providing the initial training for me to start and the following continuous support in biological studies. My dissertation work would not have been possible without the kind help from all the committee members.

I would like to thank Dr. Yan Lu, Mr. Jianjun Chen and Dr. Veeresa Gududuru for synthesizing compounds for my studies. Without their excellent work in synthesizing, I will have nothing to discover. A special thank you to the members in Dr. Andrzej Slominski's group at the University of Tennessee, Drs. Blazej Zbytek, Zorica Janjetovic, and Michael Zmijewski for their support, help and encouragement. I thank Dr. Meibohn and Dr. Nageshwar Rao Budha for their great help in drug metabolic stability studies. I thank Dr. David Hamilton for his warm help in the animal experiments. I thank Drs. Vivian S. Loveless and Christina Barrett for their great help in the tubulin colchicine site binding assay. I thank Dr. Bob M. Moore II and Dr. Steven Gurley for their help in taking microtubule images. I thank Dr. George C. Wood, Mr. Vinayagan Kannan and Dr. Wen $\mathrm{Qu}$ for their help in foumulation area. I thank Mr. Kui Zeng for his training in LC/MS. I also thank Dr. David Armbruster at the University of Tennessee Health Science Center library for his repeated and prompt editorial assistance during our publication efforts. I thank the kind help from Dr. William Seibel for ligan-based virtual screening studies. I also enjoy working with Chien-Ming $\mathrm{Li}$ in the PK studies and sharing the results of some biological studies.

I acknowledge the support of the Alma and Hal Reagan Fellowship for the academic years of 2007-2009. I really appreciate the College of Graduate Health Sciences in the University of Tennessee Health Science Center for providing the students with this fellowship as well as lots of travel awards which have supported us greatly in attending important scientific meetings. I am especially grateful for Dr. Donald B. Thomason who has promptly approved my applications for travel awards for several times. I would also like to thank the University of Tennessee Health Science Center for providing me with the opportunity to pursue graduate education.

A special thank you to my friends and colleagues, Feng Zhang, Guo Zhu, Dong Wang, Fei Ma, Yi Zhang, Li Chen, Renuka Gupte, and Zhiyong Dong for their support and wonderful company over all these years. I would also like to thank my colleagues 
who graduated before me including Wenwei Lin for their guidance and support. They really made my stay in Memphis a memorable experience.

Last but not least, I would like to thank Drs. Larry Tague and Shirley Hancock for their great help in format reviewing. Their adherence to perfectionism and great efforts help our dissertation looks much more professional. 


\begin{abstract}
Malignant melanoma is the most dangerous form of skin cancer and accounts for about $75 \%$ of skin cancer deaths. Once diagnosed at the metastatic stage, it has a very poor prognosis with a median survival rate of 6 months and a 5-year survival rate of less than 5\%. In addition, melanoma has become an important public health hazard owing to its rising incidence, which has been well documented over the past 50 years. Currently there is no effective way to treat melanoma. It is highly resistant to existing chemotherapy, radiotherapy, and immunotherapy. Over the past 30 years, only two drugs have been approved by the Food and Drug Administration (FDA) for metastatic melanoma: dacarbazine (DTIC) and interleukin-2 (IL-2). But even with these two drugs, fewer than $15 \%$ of patients have a favorable response and fewer than $5 \%$ of patients reach complete remission. On the other hand, the toxicity associated with DTIC and IL-2 is often significant, resulting in serious or life threatening side effects in many patients. In recent years, great efforts have been made in fighting metastatic melanoma. But neither combinations of DTIC with other chemotherapy drugs (e.g., cisplatin, vinblastine, and carmustine) nor adding interferon- $\alpha 2 b$ to DTIC have shown a survival advantage over DTIC treatment alone. Extensive clinical trials with a lot of antibodies and vaccines to treat metastatic melanoma have also failed to demonstrate satisfactory efficacy. Therefore, developing more effective drugs for melanoma is urgently needed.
\end{abstract}

We started our efforts in finding new drugs for melanoma by screening a large compound library. The in vitro cytotoxicity data on several melanoma cell lines led us to the discovery of three active structure scaffolds: serine amides, serine amino alcohols, and arylthiazolidine-4-carboxylic acid amides (ATCAAs). Because ATCAAs showed better selectivity between cancer cells and normal fibroblast cells, the chemists in our group focused on this scaffold and performed extensive structure modifications for structure-activity-relationship (SAR) studies. The SAR results were then used to guide further synthesis in an effort to maximize activity and selectivity. Two active compounds identified during the process were sent to the U.S. National Cancer Institute for anticancer screening using 60 human tumor cell lines. Results showed that these two compounds have extensive cytotoxic activity against all nine types of cancer cells with $\mathrm{IC}_{50}$ values ranging from $120 \mathrm{nM}$ (leukemia, CCRF-CEM cell line) to $11 \mu \mathrm{M}$ (colon cancer, HCC-15 cell line). One compound showed particularly good activity against melanoma cells $\left(\mathrm{IC}_{50}=130 \mathrm{nM} \sim 1 \mu \mathrm{M}\right.$ against all eight melanoma cell lines). I then evaluated ATCAAs inhibitory effect on melanoma colony formation and in vivo melanoma tumor growth. The in vivo data were very encouraging. One tested compound significantly inhibited melanoma tumor growth at a dose of $10 \mathrm{mg} / \mathrm{kg}$ and showed higher efficacy than did DTIC at a dose of $60 \mathrm{mg} / \mathrm{kg}$. These findings built up a strong basis for the development of novel chemotherapeutic drugs for advanced melanoma.

Furthermore, the chemists in our group also synthesized some new imidazole and imidazoline analogs by focusing on the SAR studies of the central five-member ring. Although the current compounds displayed lower potency when compared with our lead thiazolidine analogs, they may have the distinct advantage of being more stable in vivo 
with the reduced necessity of chiral separations. Some of these new compounds have activity similar to Sorafenib, an FDA-approved drug that has been tested clinically in melanoma patients.

To further expand our understanding of SARs and to potentially identify new platforms for active compounds, Dr. Li and Dr. Seibel explored a compound library from the University of Cincinnati's Drug Discovery Center. This library contains 342,910 small molecules. Based on the structure of our lead molecule, two ligand-based virtual screening approaches were used: 1) similarity search based on atom connectivity by using Scitegic Pipeline Pilot software and 2) similarity search based on molecular shape by using Schrodinger software. Results showed that these two approaches are highly complementary and lead to different active molecular structures. These structures are quite suitable for further structural modification and provide new platforms for our anticancer drug discovery efforts.

Subsequently, further lead structure optimization led to the discovery of substituted methoxylbenzoyl-aryl-thiazole (SMART) compounds. To improve solubility and to circumvent the metabolic instability brought by the thiazole ring, our team designed and synthesized a new series of analogs: 2-aryl-4-benzoyl-imidazoles (ABIs). These two classes of compounds showed great in vitro cytotoxicity against melanoma, and the $\mathrm{IC}_{50}$ of the most active compound was below $10 \mathrm{nM}$. They also showed equal potency against multi-drug resistant melanoma cells and the sensitive parent cells, indicating that these compounds can effectively overcome multi-drug resistance, which is a major cause of cancer chemotherapy failure. In vivo testing on C57BL/6 mice bearing B16-F1 melanoma allograft and on double homozygous SCID (severe combined immunodeficiency) hairless outbred (SHO) mice or athymic nude mice bearing A375 human melanoma xenograft showed these two classes of compounds significantly inhibited melanoma tumor growth. Some compounds even showed substantially better activity than did DTIC, the gold standard anti-melanoma drug. Meanwhile, preliminary toxicity studies suggested that mice can tolerate tested compounds well at effective dose levels. No sign of acute toxicity was observed from the experiments. More importantly, I identified the cellular target for ABI and SMART compounds through a series of biotechniques and molecular modeling studies. Strong experimental evidence has shown that these compounds bind to tubulin at the colchicine binding site in the $\alpha / \beta$-tubulin heterodimers to disrupt functional microtubule formation. In the meantime, I also tested the pharmacokinetic properties of some active compounds in mice together with $\mathrm{Mr}$. Chien-ming Li. With their good in vivo anti-melanoma activity and their ability to overcome multi-drug resistance, these new classes of compounds have great potential for melanoma therapy. 


\section{TABLE OF CONTENTS}

CHAPTER 1. LITERATURE REVIEW

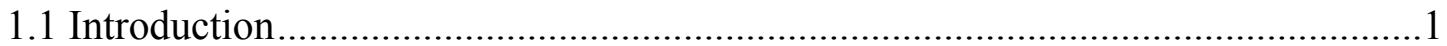

1.2 Current Treatment Agents for Metastatic Melanoma ............................................ 1

1.2.1 Chemotherapy Agent DTIC ................................................................2

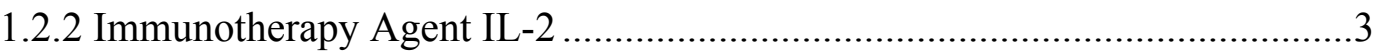

1.3 Recent Research Efforts in the Treatment for Metastatic Melanoma......................3

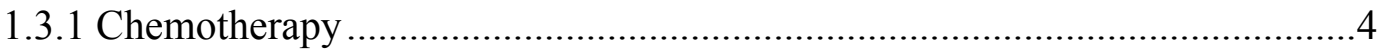

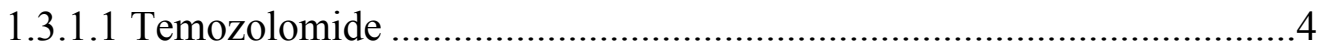

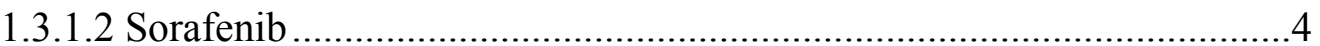

1.3.1.3 Other Single Chemotherapy Agents ................................................5

1.3.1.4 Chemotherapy Drug Combinations ................................................6

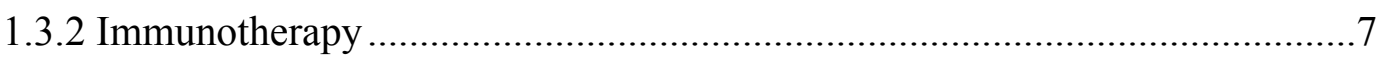

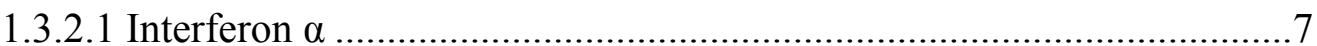

1.3.2.2 Anti-CTLA4 Antibodies: Ipilimumab and Tremelimumab..................8

1.3.2.3 Anti-Integrin Antibody: Etaracizumab ............................................9

1.3.2.4 Vaccines Based on Tumor Cells: Canvaxin, Melacine, and MVax .................................................................................. 10

1.3.2.5 Vaccines Based on Peptides: MDX-1379, Astuprotimut-R, and Others

1.3.2.6 Vaccines Based on Dendritic Cells ...................................................13

1.3.2.7 Individual Therapy Based on Activated T-Cells ...............................14

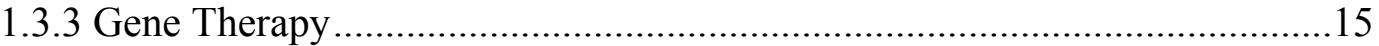

1.3.3.1 Anti-BCL2 Antisense Oligonucleotide Genasense...........................15

1.3.3.2 DNA Plasmid-Lipid Complex Allovectin-7 ......................................16

1.3.3.3 Herpes Simplex Virus Based OncoVEX ...........................................17

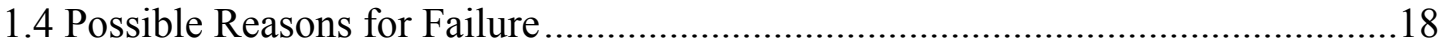

1.4.1 Reasons for Chemotherapy Failure.........................................................18

1.4.2 Barriers for Successful Immunotherapy ..................................................19

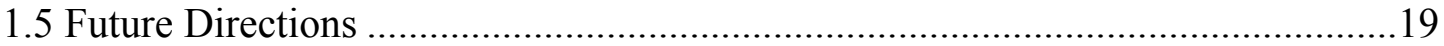




\section{CHAPTER 2. DISCOVERY OF NOVEL THIAZOLIDINE, IMIDAZOLE, AND IMIDAZOLINE ANALOGS AS CYTOTOXIC AGENTS FOR MELANOMA.}

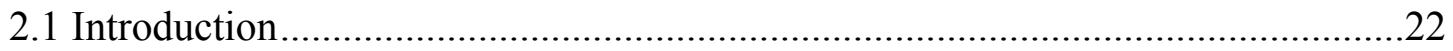

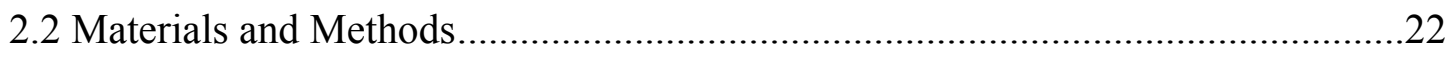

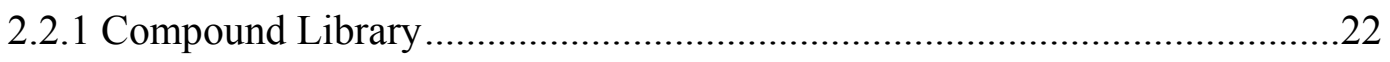

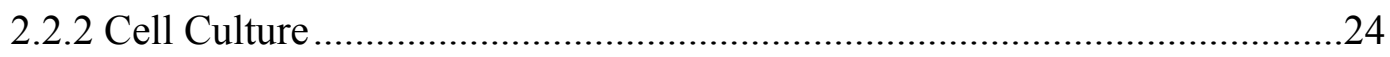

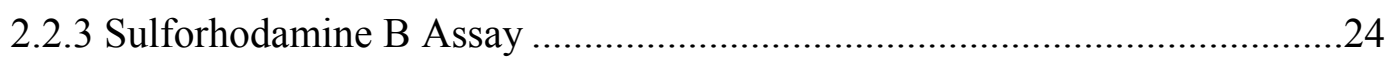

2.2.4 Lactate Dehydrogenase Release Assay...................................................24

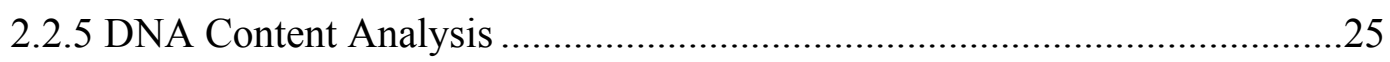

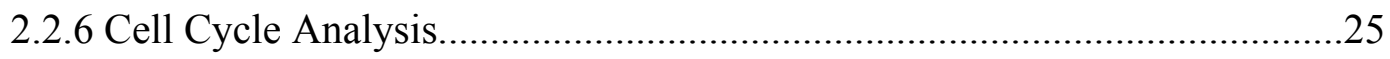

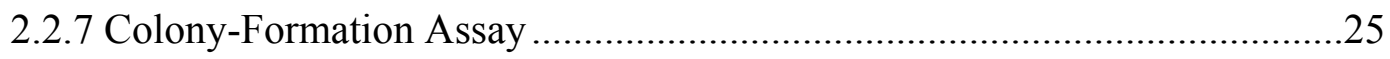

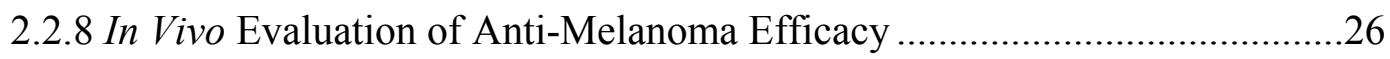

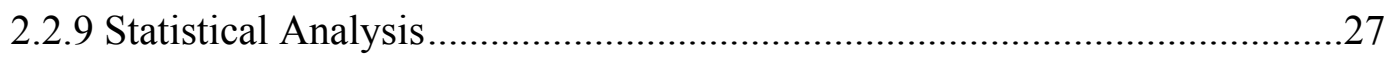

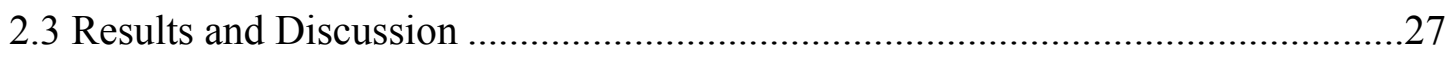

2.3.1 Identification of Lead Structures for Melanoma......................................27

2.3.2 VG-III-39 Induced DNA Degradation and LDH Release ...........................30

2.3.3 Early Stage SAR Study of Thiazolidine Derivatives ..................................30

2.3.4 Further SAR Studies with New Thiazolidine Derivatives ............................34

2.3.4.1 SAR of Different Amide Chains and Chain Length of ATCAA ..........36

2.3.4.2 SAR of Substitutions on 2-Phenyl of ATCAA .................................36

2.3.4.3 SAR of 4-Linkage of ATCAA and Thiazolidine Ring .......................37

2.3.5 SAR Summary of ATCAA for Melanoma …............................................39

2.3.6 SAR of Newly Synthesized Imidazole and Imidazoline Analogs ................39

2.3.7 Flow Cytometry Analysis of Active ATCAA Compounds .........................42

2.3.8 ATCAA Inhibited Human Melanoma Colony Formation in Soft Agar .......42

2.3.9 Antitumor Efficacy of ATCAA on A375 Xenograft Model........................42

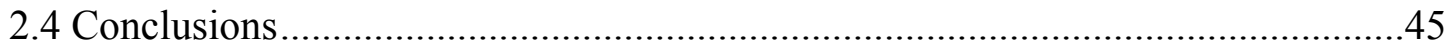

\section{CHAPTER 3. IDENTIFYING NOVEL MOLECULAR STRUCTURES FOR ADVANCED MELANOMA BY LIGAND-BASED VIRTUAL

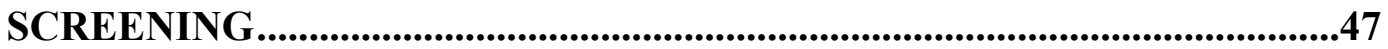

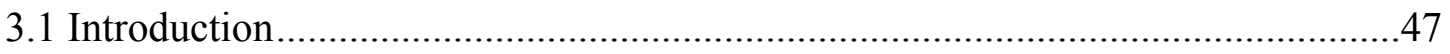

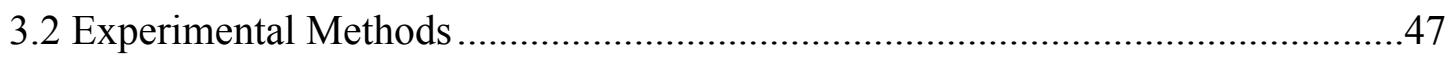


3.2.1 Validation of Connectivity Similarity Search.............................................47

3.2.2 Atom Connectivity-Based Virtual Screening ................................................49

3.2.3 Validation of Shape Similarity Search.....................................................49

3.2.4 Molecular Shape-Based Virtual Screening ................................................

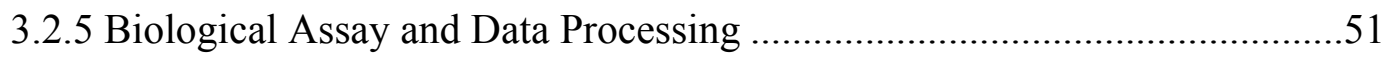

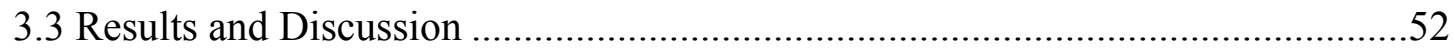

3.3.1 Validation of Connectivity Similarity Search...........................................52

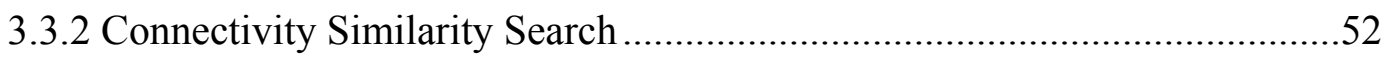

3.3.3 Additional Analog Screening Based on Active Compounds Identified from Connectivity-Based Screening .........................................................53

3.3.4 Validation of Shape Similarity Search........................................................53

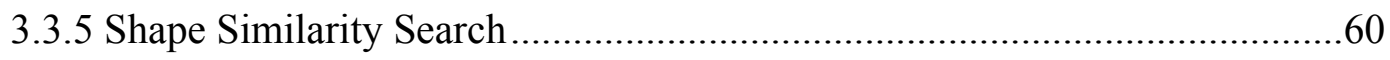

3.3.6 Comparison of Two Similarity Search Methods …………………….........67

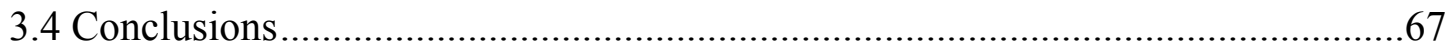

\section{CHAPTER 4. DISCOVERY AND BIO-EVALUATION OF 4-SUBSTITUTED METHOXYBENZOYL-ARYL-THIAZOLE AS NOVEL ANTI- MELANOMA AGENTS}

4.1 Introduction. .69

4.2 Materials and Methods.................................................................................

4.2.1 Compounds and Reagents........................................................................70

4.2.2 Cell Culture and Cytotoxicity Assay …………...........................................70

4.2.3 Cell Cycle Analysis...............................................................................

4.2.4 In Vitro Microtubule Polymerization Assay .................................................72

4.2.5 Tubulin Colchicine Competition Assay ………………...................................72

4.2.6 Microtubule Imaging Using Fluorescence Microscope ..................................73

4.2.7 Pharmacokinetic Studies in Mice.............................................................73

4.2.8 LC-MS/MS Method for Measuring SMART Compounds ............................74

4.2.9 Antitumor Efficacy on A375 Xenografted Athymic Nude Mice....................75

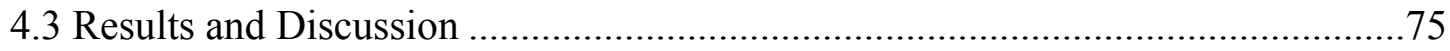

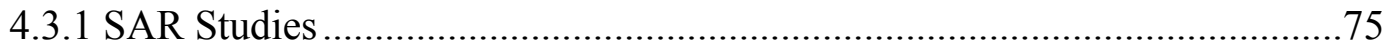

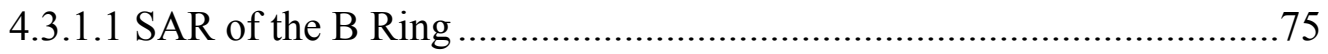

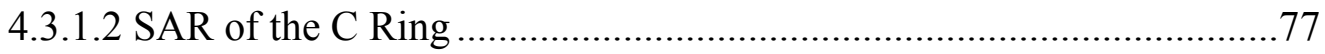

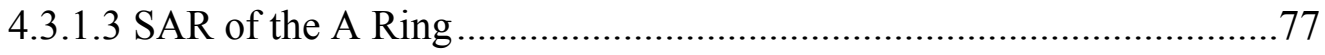


4.3.1.4 SAR of Pharmaceutically Acceptable Salt Groups to the SMARTs.

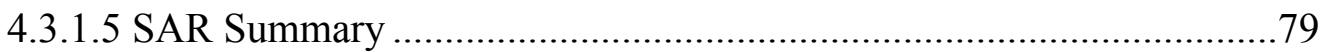

4.3.2 SMARTs Overcome Multi-Drug Resistance on Drug Resistant Melanoma Cells .... .83

4.3.3 SMARTs Target Tubulin on Colchicine Binding Site. .83

4.3.4 Pharmacokinetic Properties of SMART-100, SMART-45 and SMART211 .88

4.3.5 Antitumor Efficacy of SMARTs on A375 Xenograft Model ......................97

4.4 Conclusions

\section{CHAPTER 5. NOVEL IMIDAZOLES TARGET TUBULIN POLYMERIZATION AND OVERCOME MULTI-DRUG RESISTANCE IN METASTATIC MELANOMA}

5.1 Introduction 100

5.2 Materials and Methods 100

5.2.1 Compounds and Reagents..... 100

5.2.2 Cell Culture 102

5.2.3 In Vitro Antiproliferative Activity Assay 102

5.2.4 Colony Formation Assay in Soft Agar. 102

5.2.5 Animals 102

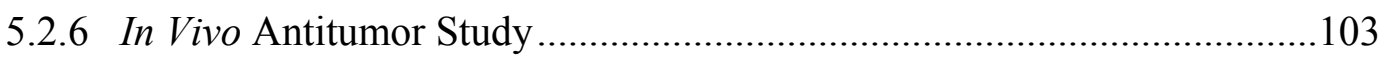

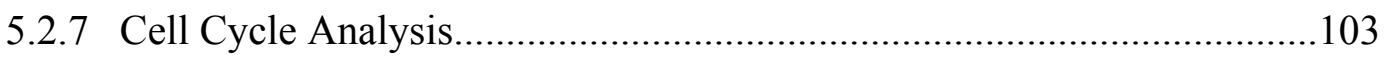

5.2.8 In Vitro Tubulin Polymerization Assay ...................................................104

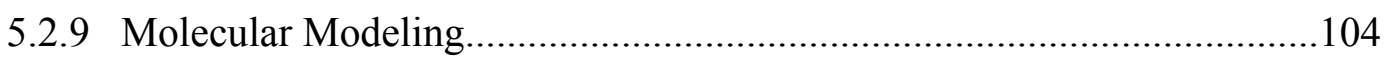

5.2.10 Tubulin Colchicine Site Binding Assay................................................104

5.2.11 Microtubule Imaging Using Immunofluorescence Microscope ................104

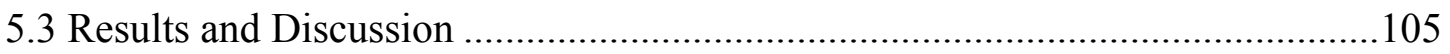

5.3.1 ABIs Have Great Potency on Melanoma Cell Lines ................................105

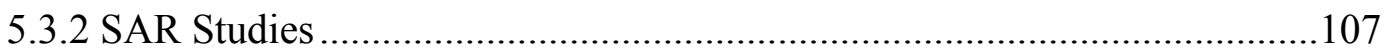

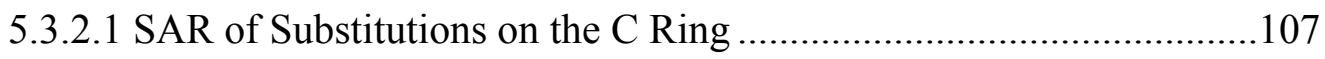

5.3.2.2 SAR of Substitutions on the A Ring ............................................ 109

5.3.2.3 SAR of Additional Substitutions on the B Ring .............................109

5.3.3 ABIs Overcome Multi-Drug Resistance on Drug Resistant Melanoma Cells 
5.3.4 ABIs Inhibit Melanoma Colony Formation in Soft Agar

5.3.5 ABIs Inhibited Melanoma Tumor Growth In Vivo.......................................112

5.3.6 ABIs Target Cell Tubulin at the Colchicine Binding Site ...........................117

5.3.6.1 Cell Cycle Analysis......................................................................117

5.3.6.2 In Vitro Tubulin Polymerization Assay .............................................122

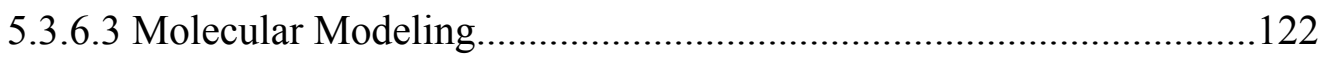

5.3.6.4 Tubulin Colchicine Site Binding Assay .............................................122

5.3.6.5 Microtubule Imaging Using Immunofluorescence Microscope ........125

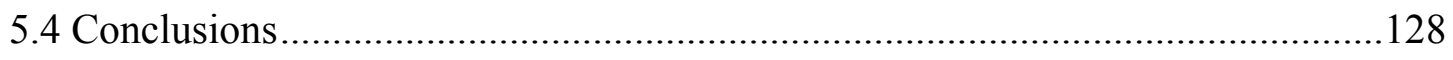

LIST OF REFERENCES............................................................................129

APPENDIX ......................................................................................................................142

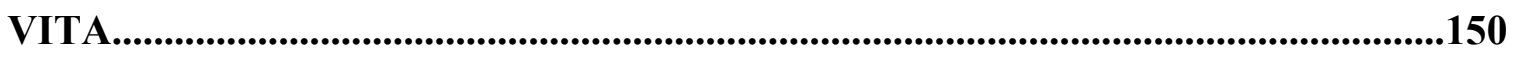




\section{LIST OF TABLES}

Table 2-1. $\quad \mathrm{IC}_{50}$ of the lead compounds, Sorafenib, DTIC and Taxol ....................... 28

Table 2-2. Cytotoxic activity of thiazolidine analogs, Sorafenib and DTIC............. 32

Table 2-3. Cytotoxic activity of ATCAA on three melanoma cell lines and a fibroblast cell line ......................................................................... 35

Table 2-4. Cytotoxic activity of thiazolidine ring-open compounds against melanoma

Table 2-5. Cytotoxic activity of imidazolines, imidazoles and comparison with Sorafenib and the lead thiazolidine compounds

Table 3-1. $\quad \mathrm{IC}_{50}$ of lead compound, eight active compounds identified from atom connectivity-based virtual screening, and newly synthesized compounds

Table 3-2. $\quad \mathrm{IC}_{50}$ of active compounds identified from further atom connectivity similarity search based on previously identified active compounds.

Table 3-3. $\mathrm{IC}_{50}$ of active compounds identified from a shape similarity search, one inactive compound, and one newly synthesized compound based on the information get from shape similarity search.

Table 4-1. Cytotoxic activity comparison of representative SMART compounds

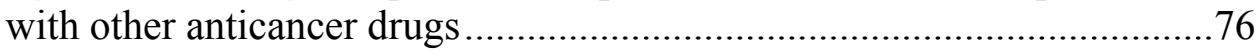

Table 4-2. In vitro cytotoxic activity of modified ATCAA compounds................... 76

Table 4-3. In vitro cytotoxic activity of SMART compounds with $\mathrm{C}$ rings modification

Table 4-4. In vitro cytotoxic activity of SMART compounds with A rings modification

Table 4-5. In vitro cytotoxic activity of SMART compounds containing hydrophilic group.

Table 4-6. SMART compounds showed equal potency against multi-drug resistant melanoma cells and parent sensitive cells

Table 4-7. Comparison of tubulin binding affinity and in vitro cytotoxic activity between SMART compounds and known tubulin interacting drugs..... 
Table 4-8. Pharmacokinetic parameters of SMART-100, SMART-45, and SMART-211

Table 4-9. Summary of mice number, mice body weight change, and tumor size change during in vivo efficacy experiment

Table 5-1. In vitro cytotoxic activity of representative $\mathrm{ABI}$ compounds and comparison with other anticancer drugs 106

Table 5-2. In vitro cytotoxic activity of $\mathrm{ABI}$ compounds with $\mathrm{B}$ and $\mathrm{C}$ ring modification 108

Table 5-3. In vitro cytotoxic activity of $\mathrm{ABI}$ compounds with A ring modification

Table 5-4. In vitro cytotoxic activity of ABI compounds with protection on $\mathrm{B}$ ring

Table 5-5. In vitro cytotoxic activity comparison of $\mathrm{ABI}$ compounds with other anticancer drugs on multi-drug resistant melanoma cell line

Table 5-6. Comparison of tubulin binding affinity and in vitro cytotoxic activity between $\mathrm{ABI}$ compounds and known tubulin interacting drugs 


\section{LIST OF FIGURES}

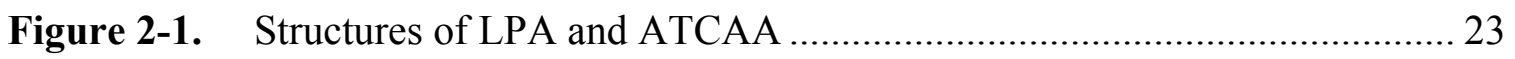

Figure 2-2. Identified active lead structures for melanoma ……………………......... 29

Figure 2-3. Compound VG-III-39 induced DNA degradation and LDH release ........ 31

Figure 2-4. Structure relationship of thiazolidine ring-open compounds ..................... 38

Figure 2-5. SAR summary of ATCAA for melanoma............................................... 39

Figure 2-6. Flow cytometry analysis of selected ATCAA compounds ....................... 43

Figure 2-7. Human melanoma A375 cells colony formation in soft agar with ATCAA treatment.

Figure 2-8. Quantified results of colony formation assay with compounds 3ad and $1 \mathrm{~b}$ treatment

Figure 2-9. In vivo anti-melanoma test of compound $1 \mathrm{~b}$ on athymic nude mice ( $\mathrm{n}=8$ per group) bearing A375 xenograft

Figure 3-1. Structure and molecular surface of lead compound, LY-1-100 48

Figure 3-2. General protocol flowchart for virtual screening validation and virtual screening

Figure 3-3. In vitro screening results for compounds identified from atom connectivity-based virtual screening.

Figure 3-4. In vitro screening results for compounds identified from further atom connectivity similarity search based on previously identified active compounds

Figure 3-5. Seeded structures in validation testing library with shape similarity ranks

Figure 3-6. In vitro screening results for compounds identified from molecular shape-based virtual screening

Figure 4-1. Structures of LPA, ATCAA and SMART …………………………..... 71

Figure 4-2. SAR summary of the SMART compounds. 
Figure 4-3. Dose response curves showed SMART compounds overcome multidrug resistant for melanoma compared with colchicine and paclitaxel.....84

Figure 4-4. Cell cycle analysis showed SMART compounds and colchicine arrested A375 cells in G2/M phase after incubation for $24 \mathrm{~h}$

Figure 4-5. Quantified cell cycle analysis results showed SMART compounds and colchicine arrested A375 cells into G2/M phase in a dose dependent manner.

Figure 4-6. SMART-100 inhibited in vitro tubulin polymerization in a dose dependent manner

Figure 4-7. Tubulin binding assay showed SMART compounds competitively bound to tubulin colchicine binding site in a concentration dependant manner.

Figure 4-8. Microscopic images of immunofluorescence labeled microtubules inside WM-164 melanoma cells showed microtubule modality was dramatically changed after compounds treatment for $18 \mathrm{~h}$

Figure 4-9. Measured plasma concentration-time profiles (mean $\pm \mathrm{SD}$ ) after bolus administration of $5 \mathrm{mg} / \mathrm{kg}$ SMART-100 to ICR mice ( $\mathrm{n}=3$ per group).... 91

Figure 4-10. Measured plasma concentration-time profiles (mean $\pm \mathrm{SD}$ ) after bolus administration of $15 \mathrm{mg} / \mathrm{kg}$ SMART-100 to ICR mice ( $\mathrm{n}=3$ per group).. 94

Figure 4-11. Measured plasma concentration-time profiles (mean $\pm \mathrm{SD}$ ) after bolus administration of $15 \mathrm{mg} / \mathrm{kg}$ SMART-45 to ICR mice ( $\mathrm{n}=3$ per group).... 95

Figure 4-12. Measured plasma concentration-time profiles (mean $\pm \mathrm{SD}$ ) after bolus administration of $15 \mathrm{mg} / \mathrm{kg}$ SMART-211 to ICR mice (n=3 per group).. 96

Figure 4-13. In vivo efficacy of SMARTs on nude mice bearing A375 melanoma xenograft tumor ( $\mathrm{n}=10$ per group) 98

Figure 5-1. Structures of SMART, ABI, and three representative ABI compounds: ABI-270, 274, and 288 .

Figure 5-2. Dose-response curves showed ABI compounds overcome multi-drug resistance for melanoma compared with other anticancer drugs.

Figure 5-3. B16-F1 melanoma colony formation assay in soft agar for ABI compounds 
Figure 5-4. Quantified representation of colony formation assay using colony counter.

Figure 5-5. In vivo studies on C57BL/6 mice bearing B16-F1 melanoma allograft for ABI-288

Figure 5-6. In vivo studies on SHO nude mice bearing A375 human melanoma xenograft for ABI-288

Figure 5-7. Cell cycle analysis of A375 cells after incubation with ABI compounds and colchicine

Figure 5-8. Quantified graphic depiction of cell cycle analysis

Figure 5-9. $\quad$ Three representative ABI compounds inhibited in vitro tubulin polymerization at a concentration of $10 \mu \mathrm{M}$

Figure 5-10. Docking of ABI-288 into tubulin crystal structure at the colchicine binding pocket.

Figure 5-11. $[3 \mathrm{H}]$ colchicine competition-binding assay confirmed ABI compounds competitively bound to tubulin at the colchicine binding site

Figure 5-12. Microscopic images of immunofluorescence labeled microtubules inside melanoma cells after compounds treatment 


\section{LIST OF ABBREVIATIONS}

$\begin{array}{ll}\mu \text { M } & \text { micro-molar per liter } \\ \text { ABI } & \text { 2-aryl-4-benzoyl-imidazole } \\ \text { AICA } & \text { 2-aryl-imidazole-4-carboxylic amide } \\ \text { AIMM } & \text { allovectin-7 immunotherapeutic for metastatic melanoma } \\ \text { AJCC } & \text { american joint committee of cancer } \\ \text { ASCI } & \text { antigen-specific cancer immunotherapeutic } \\ \text { ATCAA } & \text { arylthiazolidine-4-carboxylic acid amides } \\ \text { BCG } & \text { bacillus of calmette and guerin } \\ \text { CBDT } & \text { combination of cisplatin, DTIC, carmustine, and tamoxifen } \\ \text { CD } & \text { cluster of differentiation } \\ \text { CNS } & \text { central nervous system } \\ \text { CR } & \text { complete response } \\ \text { CTLA4 } & \text { cytotoxic t-lymphocyte antigen 4 } \\ \text { CVD } & \text { combinations of ciplatin, vinblastine, and DTIC } \\ \text { DC } & \text { dendritic cell } \\ \text { DMSO } & \text { dimethyl sulfoxide } \\ \text { DNP } & \text { dinitrophenyl } \\ \text { DTIC } & \text { dacarbazine } \\ \text { ECFC6 } & \text { extended connectivity fingerprints counts over } 6 \text { atoms } \\ \text { ECFP2 } & \text { extended connectivity fingerprints over 2 atoms } \\ \text { ECFP4 } & \text { extended connectivity fingerprints over } 4 \text { atoms } \\ \text { EDG } & \text { electron-donating-group } \\ \text { ERK } & \text { extracellular-related kinase } \\ \text { ERK-P } & \text { phosporylated ERK kinase } \\ \text { ESI } & \text { electrospray ionization } \\ \text { EWG } & \text { electron-withdrawing-group } \\ \text { FCFP4 } & \text { functional class fingerprints over 4 atoms } \\ \text { FCFP6 } & \text { functional class fingerprints over } 6 \text { atoms } \\ \text { FDA } & \text { food and drug administration } \\ \text { GM-CSF } & \text { granulocyte-macrophage colony-stimulating factor } \\ \text { GSK } & \text { glaxosmithkline } \\ \text { HDFa } & \text { human dermal fibroblast (adult) cells } \\ \text { HSV-1 } & \text { herpes simplex virus type 1 } \\ \text { IC50 } & \text { 50 percent inhibition concentration } \\ \text { ICF } & \text { infected-cell protein } \\ \text { ICR } & \text { imprinting control region } \\ \text { IFA } & \text { incomplete freund's adjuvant } \\ \text { IFN } & \text { interferon } \\ \text { IL-2 } & \text { interleukin-2 } \\ \text { LC-MS } & \text { liquid chromatography-mass spectrometry } \\ \text { LDH } & \text { lactate dehydrogenase } \\ \text { MAGE } & \text { melanoma-associated antigen } \\ \text { MAPK } & \text { mitogen-activated protein kinase } \\ & \end{array}$




$\begin{array}{ll}\text { MDR } & \text { multi-drug resistant } \\ \text { MGMT } & \text { o-6-methylguanine-dna methyltransferase } \\ \text { MHC } & \text { major histocompatibility complex } \\ \text { MITF } & \text { microphthalmia-associated transcription factor } \\ \text { MTIC } & \text { 3-methyl-(triazen-1-yl)imidazole-4-carboxamide } \\ \text { mTOR } & \text { mammalian target of rapamycin } \\ \text { NDA } & \text { new drug application } \\ \text { nM } & \text { nano-molar per liter } \\ \text { Pgp } & \text { P-glycoprotein } \\ \text { PI3K } & \text { phosphatidylinositol 3 kinase } \\ \text { PIP3 } & \text { phosphatidylinositol triphosphate } \\ \text { pM } & \text { pico-moler per liter } \\ \text { PR } & \text { partial response } \\ \text { PTEN } & \text { phosphatase and tensin homolog } \\ \text { RECIST } & \text { response evaluation criteria in solid tumors } \\ \text { SAR } & \text { structure-activity-relationship } \\ \text { SCID } & \text { severe combined immunodificiency } \\ \text { SEM } & \text { standard error of the mean } \\ \text { SEM } & \text { standard error of the mean } \\ \text { SHO } & \text { scid hairless outbreed } \\ \text { SMART } & \text { 4-substituted methoxylbenzoyl-aryl-thiazole } \\ \text { SPA } & \text { special protocol assessment } \\ \text { SRB } & \text { sulforhodamine b } \\ \text { TAA } & \text { tumor-associated antigen } \\ \text { TMZ } & \text { temozolomide } \\ \text { US } & \text { unique short region protein } \\ \text { VEGF } & \text { vascular endothelial growth factor } \\ & \end{array}$




\section{CHAPTER 1. LITERATURE REVIEW}

\subsection{Introduction}

Melanoma is a cancer derived from melanocytes which produce pigment called melanin. This cancerous transformation can be initiated from either localized pre-existing nevi or de novo, arising from the single melanocyte that is located at the junction of the epidermis (1). Under normal condition, melanocytes proliferation and migration are regulated by keratinocytes through direct cell-cell contact or soluble growth factors (2). Upon malignant transformation, melanoma cells initially have a superficial spreading (radial growth phase), followed by tumor cells invading the dermis (vertical growth phase). Then they can advance to a more aggressive metastatic phase in which the tumor cells can metastasize lymphatically or haematologically to regional and distant sites in the body (3).

Metastatic melanoma is the most dangerous form of skin cancer, accounts for about $75 \%$ of skin cancer deaths (4). If detected at an early stage, cutaneous melanoma is curable with complete surgical excision in most patients. But once the disease progresses in regional or distant sites, it is very difficult to treat. And when disseminated, it is a devastating illness. Metastatic melanoma has a very poor prognosis, with a median survival rate of 6 10 months and a 5-year survival rate of less than 5\% (5).

Melanoma has become an important public health hazard owing to its rising incidence as this has been well documented over the past 50 years. There has been a 3 to $7 \%$ worldwide annual age standardized incidence increase over the past five decades. The incidence of melanoma is rising steadily in Western populations. The number of cases has doubled in the past 20 years. The current lifetime risk is $1.9 \%$ for men and $1.37 \%$ for women (6). Melanoma is the fastest rising form of cancer among men and the second fastest rising form of cancer among women. The overall mortality from melanoma has increased because of the increase in incidence. Around 160,000 new cases of melanoma are diagnosed worldwide each year, and it is more frequent in males and Caucasians (7). According to a WHO Report, about 48,000 melanoma-related deaths occur worldwide per year. Because melanoma affects young- and middle-aged individuals, the number of years of life lost to this malignancy is high and exceeds all other adult solid tumors except testicular carcinoma (8).

\subsection{Current Treatment Agents for Metastatic Melanoma}

According to the current $6^{\text {th }}$ edition American Joint Committee of Cancer (AJCC) staging system, melanoma can be pathologically classified to the following stages: 0 , IA, IB, IIA, IIB, IIC, IIIA, IIIB, IIIC, and IV. Stage 0 is in situ melanoma. Stage I and II are growth phase of localized cutaneous melanoma with increasing thickness. Stage III has regional involvement of lymph node. Stage IV means distant metastasis. Normally stage III and IV melanoma are called metastatic melanoma. Localized melanoma is curable 
with complete surgical excision in most patients. But currently there is no effective way to treat metastatic melanoma. Only two agents in current use have been approved by the Food and Drug Administration (FDA) for metastatic melanoma: dacarbazine (DTIC) and interleukin-2 (IL-2). But even with these two drugs, only less than $15 \%$ patients have response and less than 5\% patients can reach complete remission. On the other hand, the toxicity associated with DTIC and IL-2 is often significant, resulting in serious or life threatening side effects in many of the patients treated.

In this section, I will focus on the usage and performance of DTIC and IL-2 first. Of cause surgery is often used as a treatment for melanoma whenever a surgical removal is possible. Radiation therapy is often used after surgical resection for patients with regionally advanced melanoma or for patients with unresectable distant metastases. But these two strategies of treatment for metastatic melanoma are beyond the scope of this review.

\subsubsection{Chemotherapy Agent DTIC}

Currently DTIC is the only FDA approved chemotherapy drug for metastatic melanoma. This drug gained FDA approval in May 1975 as DTIC-Dome for the treatment of metastatic melanoma. It was initially marketed by Bayer. The therapeutic effect of DTIC is believed to be produced through alkylation of DNA. While its anticancer mechanism of action is still not fully understood, DTIC is believed to be first metabolically bioactivated through a series of reactions involving CYP450. Initial demethylation to MTIC [3-methyl-(triazen-1-yl)imidazole-4-carboxamide] is followed by formation of diazomethane, the active moiety of DTIC and a potent methylating agent (9).

DTIC has produced response rates of from $15 \%$ to $25 \%$ in single-institution trials. But the overall response rate has fallen over the years from $15 \%$ to $7 \%$ and less than $5 \%$ of responses are complete in phase 3 trials. The median response durations to DTIC are 5 to 6 months. Long-term follow-up of patients treated with DTIC alone shows that only $<2 \%$ can be anticipated to survive for more than 6 years. In recent phase 3 trials that used strict response assessment criteria, the response rates with single-agent DTIC did not exceed $12 \%(10)$.

Over the past 30 years after its approval by the FDA, DTIC remains the only currently used cytotoxic drug for the treatment of metastatic melanoma. Despite its low single-agent activity, DTIC has remained the mainstay of many combination chemotherapy regimens and evaluations of resistance-reversing agents. After more than 20 years of research, DTIC is still the standard against which most new chemotherapy agents are compared (11).

As for the dose, it has been demonstrated that $850 \sim 1000 \mathrm{mg} / \mathrm{m}^{2}$ single dose of DTIC is tolerated. This single dose administration appears to deliver clinical improvements similar to those observed with multiple doses that provide the same total 
dose per cycle. This should be the reference standard for randomized trials comparing new therapies with DTIC (12).

\subsubsection{Immunotherapy Agent IL-2}

In 1998, the FDA approved intermittent high-dose bolus IL-2 based on its ability to mediate durable complete response in metastatic melanoma patients (13). IL-2 is a type of cytokine immune system signaling molecule, which is a leukocytotrophic hormone that is instrumental in the body's natural response to microbial infection and in discriminating between foreign (non-self) and self. It's a glycosylated 15,500 dalton single protein molecule. IL-2 mediates its effects by binding to IL-2 receptors, which are expressed by lymphocytes, the cells that are responsible for immunity. It is one of the only two FDA approved agents for the treatment of metastatic melanoma. Although the overall response rate is only about $15 \%$ and less than $5 \%$ of patients achieve complete remission with IL-2, its performance on melanoma is better than DTIC.

One of the major immunologic effects of IL-2 upon the immune system is to expand the total number of T-lymphocytes (CD4+ and CD8+) and to prevent lymphocyte apoptosis. Another key role of IL-2 is to provide the appropriate cytokine milieu necessary to overcome tumor-induced immune tolerance. But the exact molecular and genetic mechanisms involved in this complex interaction between the tumor and the host immune response is still largely unknown (14).

There is currently a wide spectrum of dosing schedules and regimens for IL-2 therapy, with the current standard used by most oncologists being 600,000 to 720,000 $\mathrm{IU} / \mathrm{kg} /$ dose, given at $8 \mathrm{~h}$ intervals. Although the optimal dosing schedule resulting in the best clinical response is currently unknown, previous data would suggest that the higher dose regimens as well as the number of total doses received correlates best with clinical response. Thus, several groups have begun to look at alternative dosing strategies to achieve an increased drug tolerance and tolerability profile, such as the continuous infusion of IL-2 (18 $\mathrm{mIU} / \mathrm{m}^{2} /$ day) over an extended period of $72 \mathrm{~h}(15)$. But the multiorgan toxicity of many IL-2 regimens limits its use. In addition, the tumor-killing cytotoxic T cells and natural killer cells, which are the presumed target cells for IL-2, are frequently inefficient in the tumor environment, partly due to suppressive and apoptosisinducing signals from tumor-infiltrating mononuclear phagocytes (16).

\subsection{Recent Research Efforts in the Treatment for Metastatic Melanoma}

Because of effective treatment shortage, the prognosis for metastatic melanoma patients remains very poor. Over the past 30 years, great efforts have been attempted to search for better agents or strategies to fight this deadly disease. Hundreds of different stages of clinical trials have been carried out. Although most of them have failed, some did show very promising results. Some new treatment strategies have resulted in 
paradigm shift in our approach to therapy of melanoma patients. These shining examples may markedly change our philosophy about melanoma treatment.

\subsubsection{Chemotherapy}

\subsubsection{Temozolomide}

Temozolomide (TMZ) is an orally active alkylating agent. It's a prodrug of MTIC and congener of DTIC. It has been available in the US since August 1999 and in other countries since the early 2000s. The therapeutic benefit of temozolomide depends on its ability to alkylate/methylate DNA, which most often occurs at the N-7 or O-6 positions of guanine residues. This methylation damages the DNA and triggers the death of tumor cells. However, some tumor cells are able to repair this type of DNA damage, and therefore diminish the therapeutic efficacy of temozolomide, by expressing an enzyme called O-6-methylguanine-DNA methyltransferase (MGMT) or O-6-alkylguanine-DNA alkyltransferase (17).

The single agent activity of TMZ in metastatic melanoma has been established in several phase 1 and 2 studies (18). In a randomized trial of 305 patients with advanced melanoma, TMZ showed efficacy at least equivalent to that of DTIC in terms of objective response rate, time to progression, and overall disease-free survival (19). TMZ was tolerated very well and showed an advantage in terms of improvement in the quality of life. More patients showed improvement or maintenance of physical functioning at Week 12. That trial excluded patients who had brain metastases. Because the trial design was intended to demonstrate the superiority of TMZ over DTIC, rather than equivalence, the FDA did not accept the results of that trial as grounds for approving a melanoma indication for TMZ. But in clinical practice, patients with metastatic melanoma often are treated off-label with TMZ.

TMZ has demonstrated efficacy in the treatment of variety of solid tumors, especially in brain malignancies, which is a manifestation of its far greater ability to penetrate the central nervous system (CNS). Taking into account the high rate of CNS recurrence as a site of failure after cytotoxic chemotherapy, TMZ may represent a viable alternative to DTIC, which is ineffective against melanoma CNS metastases.

\subsubsection{Sorafenib}

Sorafenib (BAY43-9006, developed by Bayer Pharmaceuticals, West Haven CT, trade name Nexavar) is an orally administered tyrosine kinase inhibitor that is a potent inhibitor of the B-Raf kinase that is frequently mutated in melanoma, as well as an inhibitor of the Vascular Endothelial Growth Factor (VEGF) receptor and other kinases. It targets the adenosine triphosphate-binding site of the B-Raf kinase and inhibits both wild-type and mutant B-Raf in vitro. Sorafenib was approved by the FDA in December 
2005 for use in the treatment of advanced renal cancer. Preclinical studies demonstrated a significant retardation in the growth of human melanoma tumor xenografts with Sorafenib. In a phase 1 study, the maximum tolerated dose of Sorafenib as a single agent was established at $400 \mathrm{mg}$ twice daily, and the most common toxicities were gastrointestinal (mainly diarrhea), dermatologic (skin rash, hand-foot syndrome), and fatigue (20).

But in further phase 2 clinical trials, Sorafenib had shown relatively little activity in metastatic melanoma when using alone. In a phase 2 trial that was conducted in 20 patients with refractory metastatic melanoma, Sorafenib showed modest activity with 1 partial response and 3 patients who achieved stable disease (21). In another phase 2 , randomized, discontinuation trial, no objective responses were achieved, and $19 \%$ of patients achieved stable disease (22).

Sorafenib combined with other chemotherapy drugs were also tested clinically. In a phase 1 and 2 study that combined carboplatin and paclitaxel with escalating doses of Sorafenib in 35 patients, a promising response rate of $31 \%$ was observed, and another $54 \%$ of patients experienced stable disease that lasted longer than 3 months. That study recently was updated to include 105 patients, and the current response rate is $27 \%$ (23). On this basis, 2 phase 3 trials have been launched to assess the efficacy of carboplatin and paclitaxel plus Sorafenib versus placebo in chemotherapy-naive patients and in previously treated patients. In December 2006, Bayer reported the combinations failed to show significant improvement of progression-free survival in melanoma patients (24).

\subsubsection{Other Single Chemotherapy Agents}

Cisplatin and carboplatin have shown modest activity as single agents in patients with metastatic melanoma. Cisplatin as single-agent therapy induced a $15 \%$ response rate with a short median duration of 3 months (25). A response rate of $19 \%$ has been reported in 26 chemotherapy-naive patients with metastatic melanoma who received carboplatin. In those patients, there were 5 partial responses, and thrombocytopenia was the doselimiting toxicity (26). In vitro studies suggested that oxaliplatin may be more active than cisplatin or carboplatin. But a small phase 2 trial in 10 patients who had received and failed prior chemotherapy produced no objective responses (27).

The nitrosoureas (carmustine, lomustine, and semustine) induce objective responses in $13 \sim 18 \%$ patients. They can cross the blood-brain barrier. But at conventional doses, little or no activity was observed against melanoma brain metastases (28). Another drawback of the nitrosureas is they induce prolonged myelosuppression. Despite these, they have been included frequently in multi-agent chemotherapy combinations, presumably for their ability to penetrate into the CNS and lack of viable alternatives for metastatic melanoma. 
The vinca alkaloids (vindesine and vinblastine) have produced responses in approximately $14 \%$ of patients (29). The taxanes have produced responses in $16 \sim 17 \%$ patients (30). All of these response rate data were obtained from phase 2 trials. None of those drugs have been evaluated as single agents in phase 3 trials. Based on the experience with DTIC, it is likely that the phase 3 trial objective response rates would be less than the rates reported from phase 2 trials. All of these drugs are rarely used currently as single-agent therapy in metastatic melanoma, but they frequently have been incorporated into combination chemotherapy and biochemotherapy regimens.

\subsubsection{Chemotherapy Drug Combinations}

Theoretically drug combination should be based on laboratory or clinical evidence of synergistic effect. But since single-agent chemotherapy regimens only provided modest activity against metastatic melanoma and lack of viable alternatives, many combination regimens have been evaluated in clinical trials. Initially two-agent combinations were tested in which DTIC was combined with a nitrosourea, vinca alkaloid, or platinum compound. In most of these trials, only $10 \sim 20 \%$ response rates were observed. There was little evidence to suggest superiority of these combinations compared with DTIC treatment alone (31-33).

In order to improve response rates, more aggressive multi-drug combinations using 3 or 4 different drugs were also tested clinically. Two most widely studied combinations are cisplatin, vinblastine, and DTIC (CVD) and the Dartmouth regimen. The later is a 4-drug combination of cisplatin, DTIC, carmustine, and tamoxifen (also called CBDT). Both combinations showed improved response rates that ranged from $30 \%$ to $50 \%$ in single-institution phase 2 studies $(34,35)$. But in further randomized phase 3 trials which involved more patients, they all showed much lower efficiency: In a randomized trial comparing CVD with single-agent DTIC that involved approximately 150 patients, the CVD arm produced a 19\% response rate compared with $14 \%$ for the DTIC arm, and there was no differences in either response duration or survival. In another randomized phase 3 trial, the CDBT combination was compared with singleagent DTIC. That cooperative group trial involved 240 patients, and the response rate was $10 \%$ for the DTIC regimen compared with $19 \%$ for the CDBT regimen $(\mathrm{P}=0.09)$. The median survival was 7 months, with no significant difference between the 2 treatment arms (11).

The main reason for such discrepancies between the findings from singleinstitution studies and the findings from large, multicenter, cooperative trials probably is selection bias. Differences in performance status, percentages of patients with visceral involvement, and number of metastatic sites easily could account for some of the observed differences. In fact, all of those factors are known to have an impact on both response rate and survival (36).

Overall, controlled trials have produced no compelling evidence to support the value of combination chemotherapy, with or without tamoxifen, in patients with 
metastatic melanoma. Toxicity was substantially greater for the combination regimen, with bone marrow suppression, nausea, emesis, and fatigue significantly more frequent with CDBT than with DTIC (11). So it is difficult to justify the use of either CVD or CBDT instead of single-agent DTIC or TMZ for the treatment of most patients with metastatic melanoma.

\subsubsection{Immunotherapy}

\subsubsection{Interferon $\alpha$}

Interferons (IFNs) are proteins made and released by the cells of most vertebrates in response to the presence of pathogens or tumor cells. They allow communication between cells to trigger the protective defenses of the immune system that eradicate pathogens or tumors. IFNs belong to the large class of glycoproteins known as cytokines. They are named after their ability to "interfere" with viral replication within host cells. IFNs have other functions: they activate immune cells, such as natural killer cells and macrophages; they increase recognition of infection or tumor cells by up-regulating antigen presentation to T lymphocytes; and they increase the ability of uninfected host cells to resist new infection by virus.

Based on the type of receptor through which they signal, human interferons have been classified into three major types. The type I interferons present in human are IFN- $\alpha$, IFN- $\beta$ and IFN- $\omega$ (37). High-dose IFN therapy using IFN- $\alpha$ was the first form of medical therapy to be approved by the FDA for use in high-risk melanoma in the adjuvant setting. Adjuvant normally means using IFN- $\alpha$ weeks after the surgical excision of the melanoma tumor. Common treatment scheme is IFN- $\alpha 2 b$ at 20 million units $(\mathrm{MU}) / \mathrm{m}^{2} /$ day intravenous injection 5 days a week for 4 weeks, then $10 \mathrm{MU} / \mathrm{m}^{2} /$ day subcutaneous injection 3 days a week for the next 48 weeks for a full year's. But IFN- $\alpha 2 b$ can also be used one month before definitive surgical lymphadenectomy. This is called 'neoadjuvant' treatment (38).

The first randomized comparison of high-dose IFN versus observation found the median relapse-free survival was 1.72 years in the high-dose IFN arm versus 0.98 year in the observation arm $(\mathrm{P}=0.0023)$ and the median overall survival was 3.82 versus 2.78 years $(\mathrm{P}=0.0237)$ respectively $(39)$. But in a later pooled analysis of more patients in more clinical trials, the relapse-free survival benefit was maintained but no overall survival benefit was seen (40).

The exact mechanism of IFN IFN- $\alpha$ 's anti-tumor efficacy is still unknown. But it was found that the STAT1/STAT3 expression ratios rose in association with IFN treatment. The clinical effects of IFN- $\alpha 2 b$ in human melanoma are also found to be inversely related to STAT3 expression (41). Induction of apoptosis has been shown to be important in vitro, if not in vivo. IFN- $\alpha$ can induce apoptosis in transformed cell lines as well as primary tumor cells (42). 
High-dose IFN is the standard of care for high-risk melanoma patients in the adjuvant setting. However, it is associated with significant toxicity. The incidence and severity of these adverse events is clearly dose-related. Consequently, there has been a great deal of interest in intermediate- and low-dose regimens administered through subcutaneous injection. However, none of the trials using intermediate or low dosing so far have been able to demonstrate any reliable benefit in terms of relapse-free survival or overall survival (43).

\subsubsection{Anti-CTLA4 Antibodies: Ipilimumab and Tremelimumab}

Cytotoxic T-Lymphocyte Antigen 4 (CTLA4) also known as CD152 (Cluster of differentiation 152) is a member of the immunoglobulin super family, which is expressed on the surface of Helper T cells and transmits an inhibitory signal to T cells that eventually shuts off the activated state. The rationale for involving this in treatment of metastatic melanoma is to block the negative signal sending by CTLA4 by using antiCTLA4 antibodies, thus reduce the sensitivity of activated T cells to negative regulatory signals and enhance the immune response of the host to tumor cells.

As of October 2007 there are two fully human monoclonal anti-CTLA4 antibodies in advanced clinical trials, one from Medarex, Inc. (Princeton, NJ) and BristolMyers Squibb (New York), called ipilimumab (MDX-010), and one from Pfizer (New York), called tremelimumab (formerly ticilimumab, CP-675,206) (44). These antibodies were produced using different types of mice with engineered immune systems, and are thus fully human, with long half-lives of $2-4$ weeks.

Ipilimumab (MDX-010) is an IgG1 monoclonal antibody. Preclinical and early clinical studies of patients with metastatic melanoma show that ipilimumab promotes antitumor activity as monotherapy and in combination with treatments such as chemotherapy, vaccines, or cytokines. The initial success with these antibodies has encouraged the rapid development of new agonistic and antagonistic antibodies that alter immune regulation, such as anti-PD-1, anti-4-1BB, anti-CD40, and anti-OX-40. On December 10, 2007, Bristol-Myers Squibb and Medarex released the results of three studies on ipilimumab (44). The three studies tested 487 patients with metastatic melanoma. Short-term tumor progression prior to delayed regression has been observed in ipilimumab-treated patients, and objective responses may be of prolonged duration. In some patients clinical improvement manifests as stable disease, which may also extend for months or years. One of the three studies failed to meet its primary goal of shrinking tumors in at least $10 \%$ of the study's 155 patients. Overall the medication produced weaker-than-anticipated efficacy on melanoma patients.

In the meantime, the side effect profile was high in the ipilimumab treated group, with the generation of autoimmune-like effects, such as diarrhea, dermatitis and effects upon the thyroid and pituitary glands. Several patients also experienced vitiligo, indicative of anti-melanocyte autoimmunity. However, the majority of the side effects were noted to be transient (except the vitiligo), improving or disappearing after the 
completion of therapy. Early clinical data suggest a correlation between these side effects and response to ipilimumab treatment and most likely reflect the drug mechanism of action and corresponding effects on the immune system (44).

Tremelimumab is an IgG2 monoclonal antibody produced by Pfizer. It blocks the binding of the antigen-presenting cell ligands B7.1 and B7.2 to CTLA-4, resulting in inhibition of B7-CTLA-4-mediated down-regulation of T-cell activation. Subsequently, B7.1 or B7.2 may interact with another T-cell surface receptor protein, CD28, resulting in a B7-CD28-mediated T-cell activation unopposed by B7-CTLA-4-mediated inhibition. Tremelimumab is thought to stimulate patients' immune systems to attack their tumors. It has been shown to induce durable tumor responses in patients with metastatic melanoma in phase 1 and phase 2 clinical studies (45).

On April 2, 2008, Pfizer announced that it has discontinued a phase 3 clinical trial for patients with metastatic melanoma after the review of interim data showed that the trial would not demonstrate superiority to standard chemotherapy (46). Studies for other tumors are planned as of October 2009, namely for prostate cancer and bladder cancer.

\subsubsection{Anti-Integrin Antibody: Etaracizumab}

Etaracizumab (also known as etaratuzumab, MEDI-522, trade name Abegrin) is an IgG1 humanized monoclonal antibody directed against the $\alpha \mathrm{V} \beta 3$ integrin. $\alpha \mathrm{V} \beta 3$ is essential for endothelial cell proliferation, maturation, and survival. When it is blocked, proliferating endothelial cells undergo apoptosis and regress. In addition, $\alpha \mathrm{V} \beta 3$ is highly expressed in melanomas and is associated with tumor growth and invasion. In preclinical studies using $\alpha \mathrm{V} \beta 3$ antagonists, inhibition of melanoma tumor growth independent of its antiangiogenic effects was reported (47). Etaracizumab has been investigated in 3 phase 1, dose-escalation studies in patients with refractory melanoma. In the phase 2 trial, 57 patients received etaracizumab alone, and 55 patients received etaracizumab plus DTIC. Etaracizumab with or without DTIC generally was well tolerated and was active in patients with metastatic melanoma. The median survival was 12.6 months for the group that received etaracizumab with DTIC and 9.4 months for the group that received etaracizumab without DTIC (48). These results encouraged people to further test this antibody in more clinical trials.

Early 2010, a study by the Etaracizumab Melanoma Study Group was reported. In this study, 112 patients were randomized to receive etaracizumab alone or etaracizumab plus DTIC. None of the patients in the etaracizumab alone study arm and $12.7 \%$ of patients in the etaracizumab plus DTIC study arm achieved an objective response. Stable disease occurred in $45.6 \%$ of patients in the etaracizumab alone study arm and $40.0 \%$ of patients in the etaracizumab plus DTIC study arm. Despite a modest increase in survival, 12.6 months in the etaracizumab alone arm, versus 9.4 months in the etaracizumab plus DTIC arm, the researchers concluded that the survival results in both treatment arms of this study were considered unlikely to result in clinically meaningful improvement over 
DTIC alone (49). At the present time, clinical development of etaracizumab has been interrupted

\subsubsection{Vaccines Based on Tumor Cells: Canvaxin, Melacine, and MVax}

The basic idea is to use tumor cell-based vaccine to stimulate and activate the host immune system to recognize, contain and eliminate cancer cells. This effect may be based on the following two pathways: direct migration of the tumor cells to the draining lymph node basin after injection, or uptaking of apoptotic or necrotic tumor cells by host dentritic cells located within the skin (50).

The most extensively studied tumor cell-based vaccine is a polyvalent, antigenrich whole cell vaccine called Canvaxin (CancerVax Corp., Carlsbad, CA). It is comprised of three melanoma cell lines that contain over 20 immunogenic melanoma tumor antigens, given intradermally every two weeks for 3 to 5 doses, followed by monthly injections for the remainder of the first year. However, several small, singleinstitution phase 1 and 2 clinical trials of Canvaxin have not yielded a striking clinical benefit in most patients when administered with BCG as an immunoadjuvant (51). But the rare complete responder to Canvaxin therapy has prompted the initiation of two multicenter phase 3 randomized trials of Canvaxin therapy in 1998. In these trials, patients who have undergone complete resection of regional (stage III) or distant (stage IV) metastatic melanoma receive postoperative adjuvant immunotherapy with Canvaxin plus Bacillus of Calmette and Guerin (BCG) or BCG alone. In April 2005, CancerVax announced the discontinuation of their phase 3 clinical trial of Canvaxin in patients with Stage IV melanoma based upon the clinical funding that it was unlikely that the trial would provide significant evidence of a survival benefit for Canvaxin-treated patients versus those receiving placebo. On October 3, 2005, CancerVax announced the discontinuation of another phase 3 clinical trial of Canvaxin in patients with Stage III melanoma base on the similar reason (52).

The second tumor cell-based vaccine that has been well studied since 1988 is Melacine. It is an allogeneic melanoma cell lysate combined with an immunologic adjuvant which is composed of a mixture of detoxified endotoxin, cell wall cytoskeleton and monophosphoryl lipid A. Early phase 1 and 2 clinical trials in 1987 and 1988 revealed some promising results, with one complete and three partial responses seen in 25 patients treated with Melacine. These results prompted the completion of seven openlabel phase 2 trials involving 139 patients with stage III/IV melanoma and a multicenter phase 3 clinical trial of Melacine versus the Dartmouth regimen. The objective response rates for all of the above studies have been between 5 and $10 \%$. Based largely upon these former results and the clinical results of other phase 3 trials, a phase 3 observation controlled trial of Melacine in melanoma patients was conducted. But the results revealed no evidence of a benefit from Melacine in patients with melanoma (53).

One very promising autologous cell vaccine is MVax which is now in active phase 3 clinical trial sponsored by AVAX Technologies, Inc. This vaccine is derived from autologous tumor cells that have been irradiated and then modified with the hapten 
dinitrophenyl (DNP) (54). In February 2004 the Journal of Clinical Oncology published an article by Dr. David Berd on the treatment of 214 Stage IIIb and IIIc melanoma patients that showed a five-year survival rate of $44 \%$. Comparison to published results of similar patients treated with surgery alone showed five-year survival figures of $22 \%$. In stage IV patients MVax has demonstrated significant response rates as a monotherapy and in published reports MVax plus adjuvant IL-2 have reported response rate of 35\% (13\% Complete Response, 22\% Partial Response). This compares to published response rates in low dose IL-2 of 3\% (55).

In October 2006, AVAX obtained a Special Protocol Assessment (SPA) agreement with the FDA for its phase 3 protocol. The SPA allows for the start of the phase 3 registration clinical trial for MVax for the treatment of patients with metastatic melanoma. In addition, the SPA addressed AVAX's ability to use a surrogate endpoint as a basis for accelerated approval. Based on this SPA, a phase 3 trial for stage IV melanoma was started on May 2007. AVAX plans to enroll up to 387 patients who will be assigned in a double-blind fashion at a 2:1 ratio to MVax or placebo vaccine. The MVax arm will consist of an initial dose of MVax followed by cyclophosphamide and then six weekly doses of MVax administered with BCG. Following vaccine administration patients will receive a specific schedule of low dose IL-2. Patients assigned to the control group will receive a treatment identical to the MVax group, except that a placebo vaccine will replace MVax. The primary endpoints of the study are best overall anti-tumor response rate and the percentage of patients surviving at least 2 years. Secondary endpoints of the study will include overall survival time, response duration, percentage complete and partial responses, progression free survival and treatment related adverse events (56).

\subsubsection{Vaccines Based on Peptides: MDX-1379, Astuprotimut-R, and Others}

The identification of tumor antigens that are present on the surface of melanoma cells is the basis for developing cancer vaccines that utilize peptide based immunotherapy. There are several melanoma differentiation antigens known involved in the synthesis of melanin and recognized by melanoma-reactive T cells, for example, gp100, MART1/Melan-A, tyrosinase, TRP-1 and TRP-2, NY-ESO-1 and the melanoma-associated antigen (MAGE) etc. One big advantage of peptide based-vaccination is that it has few toxic side effects or adverse reactions. Data suggests that most tumor cell lines established from fine needle aspiration biopsies of patients with metastatic melanoma exhibit a relatively homogeneous co-expression of MART-1 and tyrosinase, with a much more heterogeneous expression of other tumor antigens, such as gp100, NY-ESO 1 and the MAGE antigens (57).

Rosenberg and his colleagues developed a with a peptide based-vaccine using modified immunodominant peptide of the gp100 antigen, g209-2M. They used this agent vaccinated stage IV melanoma patients subcutaneously every three weeks. Following two immunizations, 10 of 11 (91\%) of patients showed a consistently high level of immunization against the native g209 217 peptide, but not against the control peptide 
g280 288. This study also demonstrated that the majority of patients immunized with the g209-2M peptide in incomplete Freund's adjuvant (IFA) consistently developed high levels of circulating immune precursors reactive against the native g209 217 peptide. Clinically, one of nine patients who received the g209 217 peptide in IFA experienced an objective cancer regression that lasted 4 months. Three of the eleven patients exhibited mixed responses with complete or partial regression of several lesions. However, all patients eventually developed progressive disease (58).

MDX-1379 vaccine consists of two gp100 melanoma peptides. These peptides are part of a protein normally found on melanocytes, or pigmented skin cells, and on melanoma cells. These melanoma peptides are recognized by cytotoxic $\mathrm{T}$ cells in melanoma patients that are positive for HLA-A2, a human immune system compatibility antigen that is expressed in approximately half of the melanoma population. Phase II data show limited evidence of MDX-1379's clinical activity although there is strong proof-ofconcept for therapeutic vaccines based on gp100 in melanoma. Medarex is currently conducting a phase 3 clinical trial with ipilimumab and MDX-1379 combination therapy in stage III and IV melanoma at multiple sites within the United States. Preliminary data showed MDX-1379 plus ipilimumab induced a modest percentage of durable response in stage IV melanoma. But autoimmune events could make the risk/benefit ratio for MDX1379 plus ipilimumab unfavorable (59).

Astuprotimut-R (also called recombinant MAGE-A3 antigen-specific cancer immunotherapeutic GSK1203486A) is a cancer vaccine consisting of a recombinant form of human melanoma antigen A3 (MAGE-A3) combined with a proprietary adjuvant with potential immunostimulatory and antineoplastic activities. Upon administration, astuprotimut-R may stimulate a cytotoxic T-lymphocyte response against tumor cells expressing the MAGE-A3 antigen, resulting in tumor cell death. MAGE-A3, a tumorassociated antigen (TAA) originally discovered in melanoma cells, is expressed by various tumor types including melanoma, non-small cell lung cancer, head and neck cancer, bladder cancer, with no expression in normal cells. MAGE-A3 protein has been in-licensed by GlaxoSmithKline (GSK) from the Ludwig Institute for Cancer Research. The proprietary immunostimulating adjuvant in this agent is composed of a specific combination of immunostimulating compounds selected to increase the antitumor immune response to MAGE-A3. Using this vaccine as intramuscular administration together with GSK's two proprietary adjuvant systems, AS15 or AS02B, they have developed a treatment regimen for cancer patients called Antigen-Specific Cancer Immunotherapeutic (ASCI).

In 2008, GSK reported a randomized, open-label phase 2 study designed to evaluate Astuprotimut-R. A total of 72 patients with measurable metastatic MAGE-A3positive cutaneous melanoma (unresectable or in transit stage III or stage IV M1a) were randomized to receive immunization with MAGE-A3 protein combined with either AS15 or AS02B as first-line metastatic treatment. Patients were to receive a maximum of 24 immunizations over four years. Clinical activity is assessed by the Response Evaluation Criteria In Solid Tumors (RECIST) criteria, the international standards for evaluation of solid tumors. Complete response (CR) and partial response (PR) i.e., disappearance or 
significant reduction of tumor, were reported in 4 patients in the AS15 group (3 CR and 1 PR) with two of these ongoing for more than two years; in the AS02B arm, 1 patient showed a partial response which lasted for 6 months. The safety profile was similar in both groups with the majority of reported adverse events being mild or moderate local or systemic reactions (60). Currently this agent still is under phase 2 clinical development for progressive metastatic cutaneous melanoma.

Because melanoma tumors are heterogeneous in their antigenic profile, it is very difficult to make vaccines that can elicit cytotoxic T-cell responses universally in all the host immune systems. Rosenberg's group analyzed 28 different peptide-based vaccines utilized in stage IV melanoma patients. A total of 381 patients were treated with 370 patients showing no response, 9 patients showing a partial response and 2 patients with a complete response, for an overall objective response rate of only $2.9 \%$. This suggested the lack of effectiveness with this single peptide based vaccination approach (61).

Next logical step is to make vaccines with multiple peptides to overcome tumor cell antigenic heterogeneity. A recent randomized phase 2 trial was performed in 26 patients with metastatic melanoma, vaccinating with four melanoma peptides. Although a high level of specific T-cell responses were noted (in $42 \%$ of the peripheral blood, $80 \%$ of sentinel lymph nodes), only three patients had a clinical response (62).

Here is the biggest issue in this area, actually many peptide based-vaccinations have resulted in a significant increase in the number of lymphocyte precursors reactive against a variety of tumor differentiation antigens by immunization with native or modified peptides. However, such immunological responses to peptide-based therapy have not translated into meaningful clinical responses for the vast majority of patients. To date, there is no study that has clearly shown a direct correlation between an immunologic response to therapy (immune cell activation) and a clinical response (regression of established tumor).

\subsubsection{Vaccines Based on Dendritic Cells}

In the normal human epidermis and dermis, dendritic cells (DC) are present as relatively immature antigen presenting cells, exhibiting relatively low levels of class II major histocompatibility complex (MHC) molecules and co-stimulatory molecules. But these immature DC are quite capable of capturing various soluble protein antigens, such as apoptotic and necrotic tumor cells and then cross-presenting such tumor-associated antigens to cytotoxic CD8+ T cells. When relatively immature DC in the skin is triggered to enter afferent lymphatic channels, this migrating pathway also initiates a phenotypic conversion that has profound immunological consequences [30]. When the DC arrives in the lymph node, it is characterized by an abundant levels of class II MHC antigens, as well as high surface levels of costimulatory molecules, such as CD40, CD54, CD80, CD83, and CD86. The matured DC is then capable of forming stable MHC class IIpeptide complexes available to activate antigen specific CD4+ T cells (63). 
To make the dendritic cell-based vaccine, the monocyte-derived, autologous DC can be pulsed in vitro with either whole irradiated, autologous tumor cells or tumor cell lysate. Once the tumor cells are "fed" to the DC in vitro, the apoptotic or necrotic cells are then processed and tumor-specific peptide antigens are then transported to the surface in both an MHC class I- and II-restricted fashion. Both immature and mature DC can be administered to patients as vaccine safely with few adverse side effects. The administration of DC via various routes of vaccination (intradermal, intranodal and intravenous) is also feasible. The first published clinical trial of DC vaccination was in 1995 and has since been followed by 98 additional clinical trials describing more than 1,000 DC-based vaccines performed in 15 different countries. Twenty-eight trials focused on patients with various advanced stages of melanoma. The safety profile was again noted to be quite remarkable, however, despite the treatment of over 1,000 patients with DC-based vaccines, the record of effectiveness have been disappointing (64).

One pretty successful DC-based trial for patients with advanced, metastatic melanoma was reported by Nestle et al. He used plastic adherent monocytes matured with a xenogenic-based $10 \%$ fetal calf serum, subsequently pulsed with either tumor cell lysate or multiple HLA-matched peptides injected intranodally. This trial involved 16 patients who were immunized on an outpatient basis. Overall, 5 of 16 patients experienced an objective response, 2 complete and 3 partial responses. The side effects were noted to be minimal in all cases, with the development of vitiligo in a few patients. One dramatic feature of this treatment was the durability of the clinical responses, with the 2 complete responders remaining free of disease for over 15 months (65).

One phase 3 clinical trial about using DC-based vaccine to treat metastatic melanoma was report by Schadendorf and colleagues recently. The trial was a prospective, randomized trial that analyzed the therapeutic effects of an autologous peptide-pulsed DC-based vaccine in patients with stage IV melanoma compared to standard chemotherapy with DTIC alone. The results revealed that the overall response in the vaccine group was $3.8 \%$ compared to $5.5 \%$ in the DTIC group, with no statistically significant differences noted in response, toxicity, overall and progression-free survival between the two groups. The median time to progression was 2.8 months versus 3.2 months respectively and the median survival was 11 months for the DTIC arm but only 9 months for the vaccine arm (66).

Although disappointed by many trials, several new avenues of DC-based immunotherapy are actively being pursued and in various stages of development, focusing on different ways to enhance the therapeutic efficacy of DC in combination with various immunoadjuvants and other anticancer agents.

\subsubsection{Individual Therapy Based on Activated T-Cells}

One very promising approach to treat metastatic melanoma is to use fully activated anti-tumor T-cells as warhead. This regimen involves the adoptive autologous transfer of highly selective tumor-reactive T-cells directed against over-expressed self- 
derived differentiation antigens after lymphodepleting chemotherapy. Rosenberg group reported in 2004 a clinical trial using this method. Cancer regression in patients with refractory metastatic melanoma with large, vascularized tumors was noted in a remarkable 18 of 35 patients (51\% response rate), including four patients with a complete regression of all metastatic disease. Such results may stem from the ability to infuse a large number of fully activated tumor infiltrating lymphocytes with anti-tumor activity into a host that is depleted of regulatory T-cells (67).

\subsubsection{Gene Therapy}

The recent developments in the field of gene transfer have advanced the use of gene therapy as a novel strategy against a variety of human malignancies. Because of its unique set of characteristics, melanoma represents a suitable target for gene therapy. Several strategies have been used by gene therapy to treat melanoma. First is to target melanoma cells to introduce "suicide" genes. Second is to transfer tumor suppressor genes. Third is to inactivate aberrant oncogene expression. Fourth is to introduce genes encoding immunologically relevant molecules. Last is to target the host's immune cells to redirect immune responses against melanoma. Clinical trials have shown the feasibility and safety of gene therapy against malignant melanoma. Although no major successes have been reported, the positive results observed in some patients support the potential for gene therapy in the management of this disease. To make gene therapy as an effective modality of treatment for malignant melanoma, better vector technology as well as increased understanding of the "bystander effect" triggered by gene transfer approaches are needed (68).

The gene therapy in our discussion is to introduce oligonucleotide or DNA sequence into host body thus to stimulate immune response to tumor cells. So it is also called DNA vaccination. This approach has been shown to induce long-lasting immunity against infectious agents and protection from tumor outgrowth in several animal models (69). Likewise, intramuscular injections of DNA (composed of naked DNA expression plasmids) into humans have also resulted in the development of an immunologic response (70). It is hypothesized that one mechanism of tumor antigen expression may involve the DNA introducing the appropriate genes into dendritic cells for subsequent processing and presentation to the host immune system. One of the obvious advantages of DNA vaccinations is that they can be administered to patients regardless of HLA-phenotype and without identifying immunogenic epitopes.

\subsubsection{Anti-BCL2 Antisense Oligonucleotide Genasense}

Genasense (Oblimersan sodium developed by Genta Inc. which is a biopharmaceutical company based in Berkeley Heights, New Jersey) is a phosphorothioate antisense oligonucleotide directed against the first six codons of the Bcl-2 messenger RNA. Binding of the drug to the mRNA recruits RNAse $\mathrm{H}$, resulting in cleavage of the mRNA. As a result, further translation is halted and intracellular protein 
concentrations of Bcl-2 decrease with time. Melanoma cell lines having Bcl-2 overexpression have been shown to enhance activity of metastasis-related proteinases, in vitro cell invasion, and in vivo tumor growth (71). Many in vitro studies have demonstrated increased sensitivity of melanoma cells to chemotherapy when combined with antisense Bcl-2 therapy (72). Genasense is the first oncology drug of its kind to directly target the biochemical pathway (known as apoptosis) whereby cancer cells are ultimately killed by chemotherapy. Genasense is believed to inhibit the production of $\mathrm{Bcl}-2$, a protein that is believed to be a fundamental cause of resistance to anticancer therapy. By inhibiting Bcl-2, Genasense may greatly improve the activity of anticancer therapy.

Encouraged by previous data, numerous clinical trials were started to evaluate the addition of oblimersan to chemotherapy in various solid tumors, including melanoma. Updated analysis from a randomized phase 3 trial, comparing DTIC combined with oblimersan, with DTIC alone in 771 patients with Stage IV or unresectable Stage III melanoma who had not previously received chemotherapy has shown a response rate of $12.4 \%$ in the former compared with $6.8 \%$ in the latter group $(\mathrm{P}=0.007)(73)$. Median progression-free survival for the oblimersan group was 2.4 months as compared with 1.6 months for the DTIC group, with a relative risk reduction of $27 \%(\mathrm{P}=0.0003)$. The median survival was increased from 7.8 months in the DTIC arm to 9 months in the oblimersan arm with a $\mathrm{P}$ value of 0.077 , which became significant when the patients with normal baseline LDH were analyzed. In terms of toxicity, no new or unexpected adverse events were observed in this study, which had not been seen with DTIC alone.

However, in May 2004, a new drug application (NDA) based on 6-months of minimum follow-up data from this trial failed to receive an affirmative vote for approval by an advisory committee to the FDA. Genta subsequently withdrew that application, and the Company has not yet made a decision regarding re-filing the U.S. application (74).

\subsubsection{DNA Plasmid-Lipid Complex Allovectin-7}

Allovectin-7 is a bicistronic plasmid formulated with a cationic lipid system containing the DNA sequences encoding HLA-B7 and beta-2 microglobulin, which together form a $\mathrm{MHC1}$ antigen. Injection of Allovectin-7 directly into tumors is designed to stimulate an immune response against both local and distant metastatic tumors. Allovectin-7 is a novel gene therapy approach for cancer with a unique mechanism of action that is fundamentally different from currently approved treatments. The following three mechanisms were believed to play roles in this agent's efficacy. Mechanism one, in HLA-B7 negative patients, a vigorous allogeneic immune response may be initiated against the foreign MHC class I antigen. Mechanism two, in all patients, $\beta 2$ microglobulin may reconstitute normal class I antigen presentation and/or increase tumor antigen presentation to the immune system. Mechanism three, in some patients, an innate pro-inflammatory response may occur that induces tumor responses following intralesional injection of the DNA/lipid complex. The final outcome of all these mechanisms is to initially cause recognition of the tumor at the local site to allow a then 
sensitized immune response to recognize un-injected tumors at distant metastatic sites (75).

In 2001, Dr. Richards and his colleagues began a high-dose, $2 \mathrm{mg}$, phase 2 trial evaluating the Allovectin-7 immunotherapeutic alone for patients with stage III or stage IV melanoma, who have few other treatment options. The high-dose phase 2 trial completed enrollment in 2003. The data showed that the trial had a total of 15 responders among the 127 patients receiving the high dose (11.8\%), with four of the patients having complete responses and 11 having partial responses. The Kaplan-Meier estimated median duration of response was 13.8 months. The Kaplan-Meier median survival was 18 months. The safety profile was excellent with no reported Grade 3 or Grade 4 adverse events associated with Allovectin-7 (76).

Allovectin-7 has been granted orphan drug designation for the treatment of invasive and metastatic melanoma by the FDA's Office of Orphan Products Development. Orphan drug designation provides U.S. marketing exclusivity for seven years if marketing approval is received from the FDA

Vical is conducting the AIMM (Allovectin-7 Immunotherapeutic for Metastatic Melanoma) trial, a phase 3 pivotal trial of Allovectin-7 as first-line therapy in approximately 375 patients with Stage III or IV recurrent metastatic melanoma in accordance with a SPA agreement completed with the FDA. The trial is being conducted at approximately 60 clinical sites worldwide. They designed the trial to include patients most likely to benefit from our treatment, and specifically excluded patients with brain or liver metastases, patients previously treated with chemotherapy, and patients with elevated lactate dehydrogenase (LDH) levels.

In January 2010 Vical announced that the company has completed enrollment of the planned 375 subjects in its multinational phase 3 trial of Allovectin-7 in patients with metastatic melanoma. Allovectin-7®'s safety profile is excellent with no drug-related serious adverse events reported to date in the phase 3 trial (75).

\subsubsection{Herpes Simplex Virus Based OncoVEX}

OncoVEX (GM-CSF) is an enhanced potency, immuneenhanced oncolytic herpes simplex virus type 1 (HSV-1). It is deleted for infected-cell protein gene 34.5 (ICP34.5), providing tumor selective replication, and ICP47 gene which otherwise blocks antigen presentation. In addition, ICP47 deletion increases unique short region protein 11 (US11) gene expression thereby enhancing virus growth and replication in tumor cells. The coding sequence for human granulocyte-macrophage colony-stimulating factor (GM-CSF) is inserted, replacing ICP34.5, to enhance the immune response to tumor antigens released following virus replication.

OncoVEX is developed by BioVex (Woburn, MA). It is a first-in-class oncolytic, or cancer destroying virus, that works by replicating and spreading within solid tumors 
(leaving healthy cells unaffected), thereby causing cancer cell death and stimulating the immune system to destroy un-injected metastatic deposits. Both modes of action have been clearly validated in the clinic, where multiple patients with metastatic disease progressing at enrollment have been declared disease free.

BioVex recently concluded a 50-patient phase 2 trial for OncoVEX (GM-CSF) as a stand-alone therapy in patients with Stage IIIc and Stage IV melanoma. The trial was designed to measure overall objective response, which is defined as a complete response, where disease is completely eliminated, or partial response, where there is a $>50 \%$ reduction in disease burden. $74 \%$ of patients who entered the study were progressing after having failed prior therapy. 13 objective systemic responses ( $26 \%$ objective response rate) were achieved including eight CRs, seven of which remain free of disease. 12 responses have so far continued for more than 6 months (ranging from 6 to more than 29 months). Responses were observed in patients with all stages of disease, including the complete resolution of un-injected visceral deposits. Adverse effects were primarily limited to transient flu-like symptoms (77).

In April 2009, BioVex Inc. announced that its OPTiM (OncoVEX Pivotal Trial in Melanoma) phase 3 study with OncoVEX (GM-CSF) in previously treated patients with Stage III and Stage IV melanoma had initiated. The study has commenced recruiting patients in the U.S. and with sites in the United Kingdom, Germany and Australia. The OPTiM trial is a multi-national, open label, randomized study designed to assess the efficacy and safety of treatment with OncoVEX (GM-CSF) as compared to subcutaneously administered GM-CSF in patients with unresectable stage III (b-c) and stage IV (M1a-c) disease. Patients will have received at least one prior therapy for active disease which includes any type of therapy including investigational drugs. A total of 360 patients will be enrolled (240 to the OncoVEX (GM-CSF) arm and 120 to the control arm). The study design was agreed with the FDA under the special protocol assessment process (78).

\subsection{Possible Reasons for Failure}

Despite an epic number of clinical trials to test a wide variety of anticancer strategies, the average survival rate for patients with metastatic melanoma remains unimproved during the past 30 years (41). Though constant clinical trials effort, although some approaches showed promising intermediate results, still no any agents for approaches have been granted a FDA approval for the treatment of metastatic melanoma. There are several reasons that may be accountable for the extremely high resistance of metastatic melanoma to current treatment modalities.

\subsubsection{Reasons for Chemotherapy Failure}

Melanoma cells are quite resistant to most chemotherapy reagents. This is associated with the specific feature of melanoma cells. In nature, these cells have low 
levels of spontaneous apoptosis in vivo compared with other tumor cell types, and they are relatively resistant to drug-induced apoptosis in vitro (4). The natural role of melanocytes is to protect inner organs from UV light, a potent DNA damaging agent. Therefore, it is not surprising that melanoma cells may have special DNA damage repair systems and enhanced survival properties (79). Moreover, recent studies showed that, during melanoma progression, it acquired complex genetic alterations that led to hyperactivation of efflux pumps, detoxification enzymes, and a multifactorial alteration of survival and apoptotic pathways. All these have been proposed to mediate the multidrug resistant phenotype of melanoma (80).

\subsubsection{Barriers for Successful Immunotherapy}

The major barrier is immunosuppressive effects activated by tumors. Tumor cell can escape immune rejection and induce immunosuppression through the following five major paths. Firstly, tumor cells may lose or down-regulate either the melanoma associated antigens or MHC molecules. Secondly, tumor cells may produce a plethora of immunosuppressive factors such as interleukin-10, VEGF and transforming growth factor. These factors create an inherently unfavorable microenvironment that limits the host immune response, in addition to tolerating the T-cell response to established tumor. Third possible reason is intrinsic inefficiency of DC whereby the appropriate co-stimulatory molecules are not being presented on the cell surface. Fourth possible reason is tumorrelated alterations in T-cell signaling and a skewing of the immune response from a Th1 (immunoactivating) to a Th2 response (immunotolerant). Lastly, the concept of tumor cell escape and immune tolerance is an exceedingly complex process. We need to further understand these mechanisms before we can have successful immunotherapy to melanoma (14).

Specifically for cancer vaccines, there are some further barriers. First is the characterization of vaccines potency and toxicity. This is especially important in the transition from phase 2 to phase 3 trials. To select a meaningful and validated end point for trials is a big challenge most of the time. Second barrier is selection of the maximum tolerated dose of cancer vaccine, particularly compared with traditional anticancer agents. Cancer vaccines are typically not very toxic. So the optimum dose often has to be based on the immune response of patients. But if the patients have previously been heavily treated with other anticancer agents, this can lead to a compromised immune system that makes it difficult to detect an evoked immune response. The third barrier is appropriate trial design and statistical data process. This is also a key part and can substantially affect final trial outcome (54).

\subsection{Future Directions}

With the rapidly rising incidence and the high resistance to current therapeutic agents, developing more effective drugs for metastatic melanoma is urgently needed. But 
before we can thoroughly understand all the major molecular pathological changes associated with melanoma malignancy, it is very difficult to reach a cure for it.

Melanoma is an extremely complicated disease, with many gene mutation and signaling pathway changes. Elevated signaling pathway in melanoma including mitogenactivated protein kinase (MAPK) pathway, phosphatidylinositol 3 kinase (PI3K)-AKT pathway, Wnt-Frizzled- $\beta$-catenin pathway, JAK/Stat pathway and $\alpha-M S H-M C 1 R$ or microphthalmia-associated transcription factor (MITF) pathway. The first two are crucial pathway accounting for melanoma malignance. Gene mutation involved in melanoma including the following oncogenes: B-raf, N-ras, akt3; tumor suppressors: CDKN2A, PTEN, p53, APAF-1, p16, p15, p19; others: Cyclin D1, MITF etc (81).

The binding of growth factors to their respective receptors leads to activation of RAS proteins. Ras will then activate Raf. Raf activate mitogen-activated protein kinase (MEK), which then act on extracellular-related kinase (ERK). Phosporylated ERK kinases (ERK-P) translocate to the nucleus and activate transcription factors, which promote cell cycle progression and proliferation. The PI3K-AKT pathway mediates cell survival signaling via growth factors. Phosphatase and tensin homolog (PTEN) inhibits growth factor signaling by inactivating phosphatidylinositol triphosphate (PIP3) generated by PI3K. Activated PI3K converts the plasma membrane lipid phosphatidylinositol 4,5-bisphosphonate to PIP3, which acts as a second messenger leading to the phosphorylation AKT and subsequent up-regulation of cell cycle, growth, and survival proteins. AKT can also up-regulate mTOR (mammalian target of rapamycin), S6K, and NFkb leading to cell growth and inhibition of apoptosis.

Knowing the huge complexity of melanoma, it's easy to understand why so many random trials of single agents or combinational treatment have failed. So targeted therapy in a systemic way based on the understanding about melanoma molecular pathology seems to be a reasonable way to fight this disease.

In learning from our efforts in the past, we must continue to challenge the current paradigms of treatment as we forge new paths to more effective treatment options. This will likely involve a multimodal approach to therapy utilizing all of the available tools in our arsenal. Several agents given in unique combinations may then synergize with standard chemotherapeutic regimens resulting in prolonged clinical responses and long term survival. Like the example of Sorafenib, its failure maybe largely because of it only blocked the Raf-MEK-ERK signaling pathway. Melanoma cells still can survive by compensatory up-regulating other survival pathway like the PI3K-AKT pathway. Then it can develop drug resistance with time by over expressing MDR gene. Ideally, if we can use drugs to synergistically block all the major pathways for melanoma cell survival and then educate our immune system to fight the tumor cells, we will have a very high chance to conquer this deadly disease.

Individualized T-cell-based therapy is a very promising approach. If combined with other suitable tumor killing agents, it maybe can improve the patient survival rate and time. But the selective tumor-reactive T-cells isolated from the patients only can be 
used for themselves. Thus there is a pretty high cost associated with it. It may not be available to all the patients in the near future.

Since all the combinational treatments are based on the performance of each individual agent, developing more effective single agents is essential. In the next several chapters, I will summary our efforts in identifying more active small molecule drugs for melanoma. That's the basis for our future treatment. It also can provide more choice for combination treatment.

For the dissertation research, my overall hypothesis was by synthesizing novel small molecule and further structure modification, we can find compounds that can inhibit melanoma tumor cell growth both in vitro and in vivo. My experimental plan was first establishing an in vitro method to test compounds activity in inhibiting melanoma cell growth. Then I would use this method to screen a large compound library to identify active lead structure. Next step was to further modify this structure to inpromve activity. Finially I would test active compounds' in vivo activity. In the meantime, compounds's pharmacokinetic properties would be also measured. 


\section{CHAPTER 2. DISCOVERY OF NOVEL THIAZOLIDINE, IMIDAZOLE, AND IMIDAZOLINE ANALOGS AS CYTOTOXIC AGENTS FOR MELANOMA*}

\subsection{Introduction}

In a continuous effort in our laboratory, our team discovered that, by replacing the glycerol backbone in lysophosphatidic acid (LPA, 1-acyl-sn-glycerol-3-phosphate), the resulting 2-arylthiazolidine-4-carboxylic acid amides (ATCAAs) were potent cytotoxic agents for melanoma (82-87). Two of earlier derivatives ( $2 R S, 4 R)$-2-phenyl-thiazolidine4-carboxylic acid octadecylamide 1a and ( $2 R S, 4 R)$-2-(4-acetamidophenyl)-thiazolidine4-carboxylic acid hexadecylamide $1 \mathrm{~b}$ (Figure 2-1) were sent to the U.S. National Cancer Institute for anticancer drug screen using 60 human tumor cell lines (NCI-60). The NCI60 screening data indicated that compounds $1 \mathrm{a}$ and $1 \mathrm{~b}$ strongly inhibited the growth of all nine types of cancer cells with $\mathrm{IC}_{50}$ values ranging from $120 \mathrm{nM}$ (leukemia, CCRF-CEM cell line) to $11 \mu \mathrm{M}$ (colon cancer, HCC-15 cell line). 1b was very potent against melanoma $\left(\mathrm{IC}_{50}=130 \mathrm{nM} \sim 1 \mu \mathrm{M}\right.$ against all eight tumor cell lines) and prostate cancer $\left(\mathrm{IC}_{50}=170 \sim 270 \mathrm{nM}\right.$ against two tumor cell lines). Based on these preliminary cytotoxic screenings, extensive structure-activity relationship (SAR) and in vivo studies were carried out. In this chapter, I will discuss the biological evaluation and SAR studies of ATCAA analogues based on $1 \mathrm{a}$ and $1 \mathrm{~b}$ for both melanoma. These analogs were further assessed for their ability to inhibit melanoma colony formation. The in vivo efficacy of compound $1 \mathrm{~b}$ was tested in a xenograft model using human A375 melanoma tumors. This class of compounds showed potent antitumor activity and selectivity, which could represent the basis for their development into novel chemotherapeutic drugs.

\subsection{Materials and Methods}

\subsubsection{Compound Library}

Ninety-one compounds were selected to form a library for biological testing. Their activity was compared with the existing drugs, Taxol and DTIC (Sigma-Aldrich, Inc., St. Louis, MO). In addition, Sorafenib (Bay43-9006) has recently been used extensively in clinical trials for advanced melanoma and is well tolerated in patients (22). Hence, Dr. Wei Li also synthesized Sorafenib based on published procedures (88), and

*Portions of this chapter adapted with permission from the following:

1. Li W, Wang Z, Gududuru V, et al. Structure-activity relationship studies of arylthiazolidine amides as selective cytotoxic agents for melanoma. Anticancer Res 2007;27:883-8.

2. Li W, Lu Y, Wang Z, et al. Synthesis and antiproliferative activity of thiazolidine analogs for melanoma. Bioorg Med Chem Lett 2007;17:4113-7.

3. Chen J, Wang Z, Lu Y, et al. Synthesis and antiproliferative activity of imidazole and imidazoline analogs for melanoma. Bioorg Med Chem Lett 2008;18:3183-7.

4. Lu Y, Wang Z, Li CM, et al. Synthesis, in vitro structure-activity relationship, and in vivo studies of 2arylthiazolidine-4-carboxylic acid amides as anticancer agents. Bioorg Med Chem 2010;18:477-95. 
<smiles>CCCCCCC(=O)OC[C@@H](O)COP(=O)(O)O</smiles>

LPA

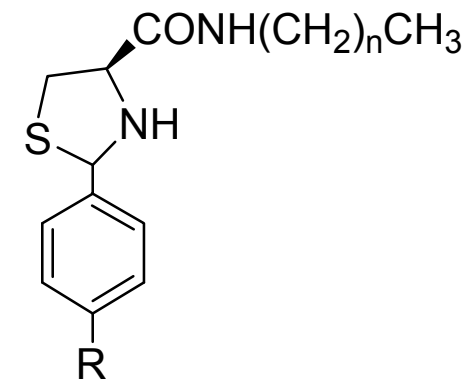

ATCAA

1a $n=17, \mathrm{R}=\mathrm{H}$

1b $n=15, \mathrm{R}=\mathrm{NHCOCH}_{3}$

Figure 2-1. Structures of LPA and ATCAA. 
used its activity as a standard to assess our lead compounds. Thiazolidine analogues were synthesized by Dr. Yan Lu. Imidazole and imidazoline compounds were synthesized by Mr. Jianjun Chen. I worked for the biological part.

\subsubsection{Cell Culture}

SK-MEL-188 cells were derived from primary tumors and cultured in Ham's F10 medium (Gibco Invitrogen, Inc., Grand Island, NY) plus 5\% fetal bovine serum (FBS) (Cellgro Mediatech, Inc., Herndon, VA) and 1\% antibiotic/antimycotic mixture (SigmaAldrich, Inc., St. Louis, MO) (89). WM-164 cells were derived from metastatic tumors and were a gift from Dr. Meenhard Herlyn (Wistar Institute, Philadelphia, PA). A375 cells and B16-F1 cells were purchased from ATCC (American Type Culture Collection, Manassas, VA). Both WM-164 and human dermal fibroblast cells (purchased from Cascade Biologics, Inc., Portland, OR) were cultured in DMEM (Cellgro Mediatech), supplemented with 5\% FBS, $1 \%$ antibiotic/antimycotic mixture and bovine insulin (5 $\mu \mathrm{g} / \mathrm{mL}$; Sigma-Aldrich Inc., St. Louis, MO). Human dermal fibroblast (adult) cells (HDFa) were used as a control to examine the effect of compounds on the stromal environment. Thus, the effect of drugs on melanoma cells versus fibroblasts would document their potential selectivity.

\subsubsection{Sulforhodamine B Assay}

Cells were seeded into 96-well plates at 5000 cells/well. After $12 \mathrm{~h}$, media were changed and serial dilutions of compounds were added $(1 \mathrm{nM} \sim 100 \mu \mathrm{M})$. Cells were incubated with each compound for $48 \mathrm{~h}$. Fresh media containing the testing compound were changed every $24 \mathrm{~h}$. Thereafter, total cell protein corresponding to cell numbers (both viable and non-viable cells) was measured using the sulforhodamine B (SRB) assay according to the manufacturer's protocol (Sigma-Aldrich Inc., St. Louis, MO) $(79,90)$. Briefly, cells were fixed with trichloroacetic acid, washed, and incubated with sulforhodamine B for $30 \mathrm{~min}$. After another wash, dye incorporated into the cells was solubilized and measured using a plate reader at $565 \mathrm{~nm}$.

\subsubsection{Lactate Dehydrogenase Release Assay}

SK-MEL-188 cells were seeded at a density of 5000 cells per well in 96-well flatbottom microtiter plates. Media were changed after $12 \mathrm{~h}$, and the testing compound was added at serial dilutions. Media were changed with fresh compound every $24 \mathrm{~h}$. After incubation for $48 \mathrm{~h}$, supernatants were collected and transferred to a new plate. Lactate dehydrogenase (LDH) in supernatants was measured using Cytotox 96 Non-Radioactive Cytotoxicity Assay (Promega, Madison, WI). Briefly, substrate mix solution (containing tetrazolium salt) was added to each well and incubated at room temperature for $30 \mathrm{~min}$. At the end of incubation, stop solution was added to each well and the plate was read at $490 \mathrm{~nm}$. Cytotoxicity of compounds was calculated using the following formula: \% 
cytotoxicity=(experimental LDH release/maximum LDH release). Experimental LDH release was calculated by subtracting the absorbance corresponding to the media alone from the absorbance corresponding to control or compound-treated cells. Maximum LDH release was calculated by subtracting the absorbance corresponding to the media with added lysis solution from the absorbance corresponding to cells lysed with lysis solution.

\subsubsection{DNA Content Analysis}

Flow cytometry analysis was performed as described elsewhere (91). Briefly, SKMEL-188 cells were seeded in $10 \mathrm{~cm}$ Petri dishes at a density of $10^{6}$ cells per dish; at 12 $\mathrm{h}$ the medium was changed to $5 \%$ FBS with different concentrations $(0,6$ and $60 \mu \mathrm{M})$ of the testing compound. Cells were incubated for $48 \mathrm{~h}$ and media with compounds were changed every $24 \mathrm{~h}$. Cells were trypsinized, washed with PBS, and fixed in ice-cold $70 \%$ ethanol. Ethanol was removed by centrifugation and cells were incubated in phosphatecitrate buffer $\left(0.2 \mathrm{M} \mathrm{Na}_{2} \mathrm{HPO}_{4}, 4 \mathrm{mM}\right.$ citric acid, $\mathrm{pH}$ 7.8) for $1 \mathrm{~h}$. Finally, cells were centrifuged and $1 \mathrm{~mL}$ of propidium iodide solution $(50 \mu \mathrm{g} / \mathrm{mL})$ and RNAse $(0.1 \mathrm{mg} / \mathrm{mL})$ in PBS was added (92). Samples were shaken for $30 \mathrm{~min}$ and analyzed with a FACS Calibur cytometer (Beckton Dickinson, San Diego, CA). Data were analyzed and graphs prepared using the Modfit 2.0 program (Verity Software House, Topsham, ME).

\subsubsection{Cell Cycle Analysis}

Flow cytometry analysis was performed to study cell cycle phase distribution. Briefly, A375 cells $\left(4 \times 10^{6}\right)$ were seeded in $10 \mathrm{~cm}$ dishes; Cells were synchronized using $0.5 \%$ charcoal-stripped DMEM medium for $72 \mathrm{~h}$. After that, the medium was changed to the media containing $10 \%$ FBS with different concentrations $(0,1,5,10$ and $20 \mu \mathrm{M})$ of testing compounds. Cells were incubated for additional $24 \mathrm{~h}$. All cells were collected by trypsinization. Cell pellets were washed by PBS. The supernatant was discarded, and the pellets were fixed in ice-cold $70 \%$ ethanol at $4{ }^{\circ} \mathrm{C}$ overnight. Ethanol was removed by centrifugation and cell pallets were washed with PBS twice. $1 \mathrm{~mL}$ of PBS containing 100 $\mu \mathrm{g} / \mathrm{mL}$ RNAase A was then added and cell pallets were incubated for $1 \mathrm{~h}$. Cells were centrifuged and re-suspended in $1 \mathrm{~mL}$ of PBS containing propidium iodide $(50 \mu \mathrm{g} / \mathrm{mL})$. Cell cycle analysis was conducted with a FACS Calibur Autometer (Beckton Dickinson, San Diego, CA). Data were analyzed and graphs were prepared using the Modfit 2.0 program (Verity Software House, Topsham, ME).

\subsubsection{Colony-Formation Assay}

To measure colony formation and growth of melanoma, A375 cells were plated at a colony-forming density (2000 cells per well on 6-well plates). Cells were grown in DMEM supplemented with FBS (Atlanta Biologicals, Lawrenceville, GA) and an antibiotic-antimycotic solution (Sigma, St. Louis, MO) at $37^{\circ} \mathrm{C}$ in an atmosphere of $95 \%$ air and $5 \% \mathrm{CO}_{2}(93)$. Cells were treated with compounds $1 \mathrm{~b}$ and $3 \mathrm{ad}$ at different 
concentrations $(2,20$, and $100 \mu \mathrm{M})$. Compounds were added to the medium from $1 \mathrm{mM}$ DMSO stock solutions, and corresponding dilution of DMSO was used as negative (vehicle) control. Cells were grown for 14 days, and colonies were stained with $0.1 \%$ crystalline blue for $30 \mathrm{~min}$ and rinsed with distilled water to remove excess dye. Plates were photographed, and the number of colonies was measured by Artek 880 Automated Colony Counter (Artek Systems Corporation, Farmingdale, NY).

\subsubsection{In Vivo Evaluation of Anti-Melanoma Efficacy}

Compound $1 \mathrm{~b}$ was dissolved into a co-solvent system that was composed of $80 \%$ Tween 80 (Sigma-Aldrich, St. Louis, MO) and 20\% Captex 200 (Abitec Corporation, Columbus, OH). Dacarbazine (DTIC) (Sigma-Aldrich, St. Louis, MO) was dissolved in saline solution. Male athymic nude mice aged 4-5 weeks were purchased from Harlan Laboratories (Harlan Laboratories, Indianapolis, IN). The laboratory housing the animals met all Association for Assessment and Accreditation and Laboratory Animal Care specifications. All of the procedures were conducted in accordance with the guidelines of our Institutional Animal Care and Use Committee.

A375 cells in a logarithmic growth phase were prepared in FBS-free DMEM medium (Cellgro Mediatech) at a concentration of $5 \times 10^{7}$ viable cells $/ \mathrm{mL}$ and placed on ice. The cell suspension was mixed with BD Matrigel (BD Biosciences, Waltham, MA) at a 1:1 ratio. This cell suspension $(100 \mu \mathrm{L})$ was injected subcutaneously in the right dorsal flank of each mouse. When tumor sizes reached about $150 \mathrm{~mm}^{3}$, about 7 days after cell inoculation, all mice bearing tumors were divided into control and treatment groups based on tumor size ( $n=8$ per group). Each group had similar average tumor size. Mice in control groups were injected intraperitoneally with $50 \mu \mathrm{L}$ vehicle solution only (negative control) or DTIC at $60 \mathrm{mg} / \mathrm{kg}$ (positive control) once daily. Tumor volume was measured twice weekly with a Traceable ${ }^{\circledR}$ electronic digital caliper (Fisher Scientific, Inc.,

Pittsburgh, PA) and calculated using the formula $a \times b^{2} \times 0.5$, where $a$ and $b$ represented the larger and smaller diameters, respectively (94). Tumor volume was expressed as cubic millimeters. Data were expressed as mean \pm SE for each group and plotted as a function of time. Percentage tumor reduction at the conclusion of the experiment (22 days after initiating treatment) was calculated from the formula $100-100 \times\left[\left(\mathrm{T}-\mathrm{T}_{0}\right) /\left(\mathrm{C}-\mathrm{C}_{0}\right)\right]$, where $\mathrm{T}$ represents mean tumor volume of a treated group on a specific day, $\mathrm{T}_{0}$ represents mean tumor volume of the same group on the first day of treatment, $\mathrm{C}$ represents mean tumor volume of a control on a specific day, and $\mathrm{C}_{0}$ represents mean tumor volume of the same group on the first day of treatment (95). Animal activity and average body weight of each group were monitored during the entire experiment period to assess compound toxicity. At the end of treatment, all mice were euthanized by $\mathrm{CO}_{2}$ followed by cervical dislocation, and tumors were harvested for further studies. 


\subsubsection{Statistical Analysis}

Data are presented as mean $\pm \operatorname{SEM}(n=6 \sim 8)$. $\mathrm{IC}_{50}$ (i.e., concentration that inhibited cell growth by $50 \%$ of untreated control) values were obtained by nonlinear regression analysis (GraphPad Prism, San Diego, CA). Statistical analyses were performed with the Student's $t$-test $(* p<0.05, * * p<0.001)$.

\subsection{Results and Discussion}

\subsubsection{Identification of Lead Structures for Melanoma}

In the first step of lead identification, cells were exposed to each compound at 10 $\mu \mathrm{M}$ to identify those which are active for both SK-MEL-188 and WM-164 cell lines. The $\mathrm{IC}_{50}$ values of the seven most active compounds with $50 \%$ or more growth inhibition at $10 \mu \mathrm{M}$ were subsequently measured on both cancer cell lines and on the control cells (fibroblast cells). Activities of several compounds with similar structures to the active compounds were also measured in order to understand the structure-activity relationships (SAR). Sorafenib, DTIC and Taxol were assayed in the same way for comparison and served as internal quality controls in the different assay batches. The results for all three cell types are summarized in Table 2-1.

Overall, three basic structures were found very active in inhibiting both melanoma cell lines: (A) serine amides (e.g. VG-III-15), (B) serine amino alcohols (VG-II-197), and (C) arylthiazolidine-4-carboxylic acid amides (ATCAA, e.g., VG-III-39) (Figure 2-2). Analogs of (A) and (B) did not possess acceptable selectivity for cancer cells. Thiazolidine derivatives $(\mathrm{C})$ displayed the highest potency and selectivity with $\mathrm{IC}_{50}$ values in the nano-mole range for cancer cells (Table 2-1, VG-III-39). Not surprisingly, data from this in vitro assay indicated that DTIC is inactive $\left(\mathrm{IC}_{50}>100 \mu \mathrm{M}\right)$ against both cancer cell lines due to the lack of bio-activation. This is consistent with the currently proposed mechanism of action for DTIC, as well as findings by Daidone and co-workers (96). Clearly, in vivo assay is needed to assess the true activity of DTIC and similar structures. While Taxol is outstanding in inhibiting cancer cell growth, it is not effective in killing melanoma cells (cytostatic). In contrast, all the lead compounds from these preliminary studies killed the cells completely at higher concentrations. The mechanisms of action of our compounds are clearly different from that of DTIC and Taxol.

Sorafenib effectively killed all the melanoma cells at high concentrations, similar to that of our lead compounds. When measured by $\mathrm{IC}_{50}$ values, VG-III-39 is about 10 times as potent as Sorafenib. Its selectivity, defined as the ratio of $\mathrm{IC}_{50}$ in fibroblast cells over $\mathrm{IC}_{50}$ in melanoma cells, is moderate when compared with that of Sorafenib (10 vs. larger than 3). Together with the clinical finding that Sorafenib has low toxicity and is well tolerated by patients (22). The potency and selectivity of Sorafenib provide an excellent standard to assess the activities of our compounds and represent the future goal for optimization of our lead structures. 
Table 2-1. $\mathrm{IC}_{50}$ of the lead compounds, Sorafenib, DTIC and Taxol.

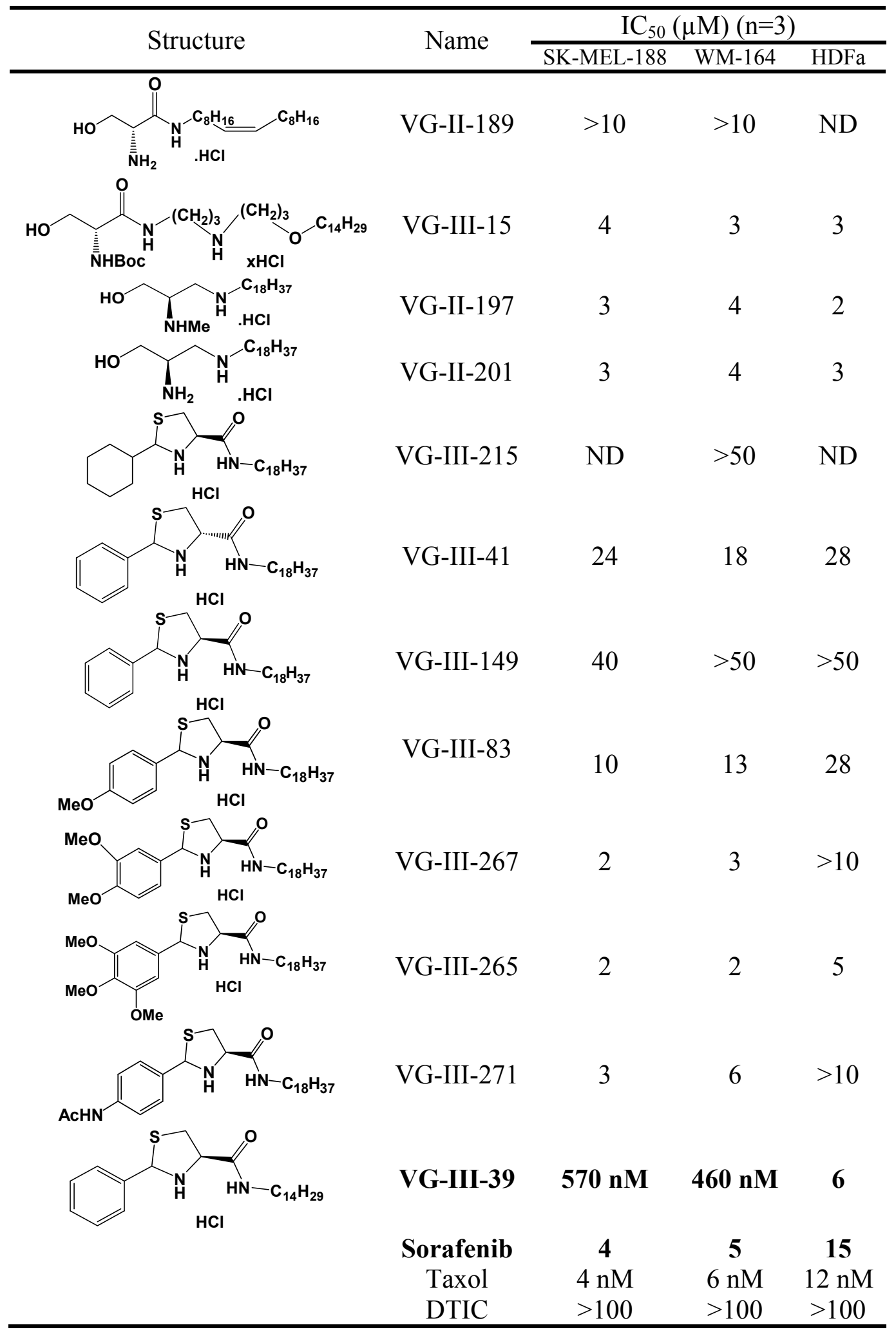

ND: not determined. 
<smiles>[R]NC(=O)C(N)CO</smiles>

A

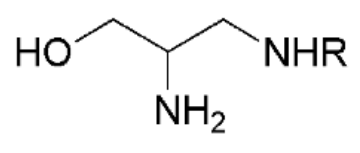

B

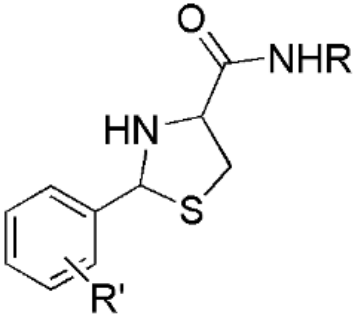

C

Figure 2-2. Identified active lead structures for melanoma.

Several important SARs were revealed in examining the 91-member library. Firstly, amides were less active than the corresponding alcohols (Figure 2-2A and B respectively). Heteroatoms on the side chain substantially increased the potency (VG-III15), but unsaturation on the side chain had the opposite effect (VG-II-189). Secondly, an alkyl amine (VG-II-197) was about equipotent to the un-substituted amine (VG-II-201), suggesting that cyclization of the amine and alcohol may be possible. However, none of the open chain compounds displayed acceptable selectivity against cancer cells. Thirdly, the presence of the aromatic ring in the heterocyclic compounds improves selectivity and cytotoxicity of structure C (Figure 2-2) (VG-III-215 vs. VG-III-149). Fourthly, the potency of the compounds depended strongly on the chirality at C-4 position (VG-III-41 vs. VG-III-149), the substitution on the aromatic ring (VG-III-149 vs. VG-III-265), and the chain length (VG-III-39 vs. VG-III-149). With a similar C18 chain, the $S$-isomer at the C-4 position (VG-III-41) showed higher potency than the $R$-isomer (VG-III-149). More electron donating substitutions on the aromatic ring progressively increased the potency of analogs of structure C (VG-III-149 vs. VG-III-83, VG-III-267, and VG-III265). N-substitution with the $\mathrm{C} 14$ chain was much more potent and selective against cancer cells than that of the C18 chain compound (VG-III-39 vs. VG-III-149). Clearly, further structural optimization is warranted and will likely improve both potency and selectivity for this class of compounds against melanoma.

It is interesting to compare the activity in melanoma with that in prostate cancer for these compounds. In general, their activities are consistent in these two types of cancer cells, demonstrating potentially broad anticancer properties for ATCAA analogs. Compounds that are active or inactive in prostate cancer also displayed similar behavior in melanoma, but there are a few exceptions. For example, although VG-III-149 (C18 chain length) showed excellent activity $\left(\mathrm{IC}_{50}<3 \mu \mathrm{M}\right)$ against prostate cancer cells, it was not active in both melanoma cell lines $\left(\mathrm{IC}_{50}>40 \mu \mathrm{M}\right)$. In addition, for compounds which are active in both cancer types, their relative potency and selectivity are not the same. For example, in prostate cancer studies, a compound with a C14 side chain was found to be slightly less active than that with a C18 chain. Substitutions on the aromatic ring with mono-, di- or tri-methoxyl groups were found to be favorable in prostate cancer cell lines. Both the potency and selectivity increased as more methoxyl substitutions were added to the aromatic ring (82). In melanoma, however, methoxyl substitutions did not have significant selectivity benefits when compared with nonsubstituted analogs (e.g. VG-III- 
83 vs. VG-III-265). A C14 chain displayed much better activity and selectivity than C18 chain compounds (e.g. VG-III-39 vs. VG-III-265). Interestingly, Taxol behaves very differently in prostate cancer cells and melanoma cells. While Taxol killed all the prostate cancer cells, it only killed about half of the melanoma cells when compared with controls based on our in vitro assay. This may suggest different oncogenic properties between these two types of cancer.

\subsubsection{VG-III-39 Induced DNA Degradation and LDH Release}

To examine more closely the cell death induced by our compounds, I measured lactate dehydrogenase (LDH) release by colorimetric assay and analyzed DNA content by flow cytometry.

LDH is a stable cytosolic enzyme that is released upon cell lysis. Treatment of cells with VG-III-39 resulted in a dramatic dose-dependent increase of LDH released to the cellular environment (Figure 2-3A).

Analysis of the DNA content of control cells (Figure 2-3B) showed peaks corresponding to the cells in G1/0-cell phase (62\%), S-phase (32\%) and G2/M-phase (6\%), a typical distribution of cells in culture (92). Treatment of cells with VG-III-39 at 6 $\mu \mathrm{M}$ and $60 \mu \mathrm{M}$ led to the disappearance of peaks corresponding to viable, proliferating cells and the appearance of single peaks corresponding to debris/degraded DNA.

\subsubsection{Early Stage SAR Study of Thiazolidine Derivatives}

The ability of thiazolidine derivatives to inhibit the growth of two melanoma cancer cell lines and fibroblast cells is summarized in Table 2-2. Sorafenib (Velcade) has been used extensively in clinical trials for melanoma. Hence I selected this compound and DTIC as reference standards to assess the activity of our compounds. At this early stage, all compounds were used as a diastereomeric mixture if they contain chiral centers in order to select the most promising compounds for further development.

Examination of antiproliferative effects for a variety of substitutions on the phenyl ring revealed the chain-length dependence for these compounds $(1 \sim 5,6 \sim 9,10 \sim 13$, 14 19). Short chain length such as a C10 chain (e.g., compound 2, 6, 10) displayed low potency for both cancer cells and fibroblast cells. As chain length increased, potency increased, as well as toxicity as measured on fibroblast cells except when the acetyl amino group was substituted on the phenyl ring (compound 14 17). Both C15 and C16 chains with this substitution displayed both high potency and high selectivity against cancer cells, with an $\mathrm{IC}_{50}$ for melanoma cells as low as $600 \mathrm{nM}$ (compound 17). Further chain increases, however, reduced potency and selectivity. At a chain length of C18 (compound 19), the $\mathrm{IC}_{50}$ value was higher than $10 \mu \mathrm{M}$ for all three cell lines. Interestingly, adding either a cis- or trans- double bond in the $\mathrm{C} 18$ side chain restored potency dramatically (compound $20 \mathrm{Z}$ and $20 \mathrm{E}$ ), demonstrating that both length 

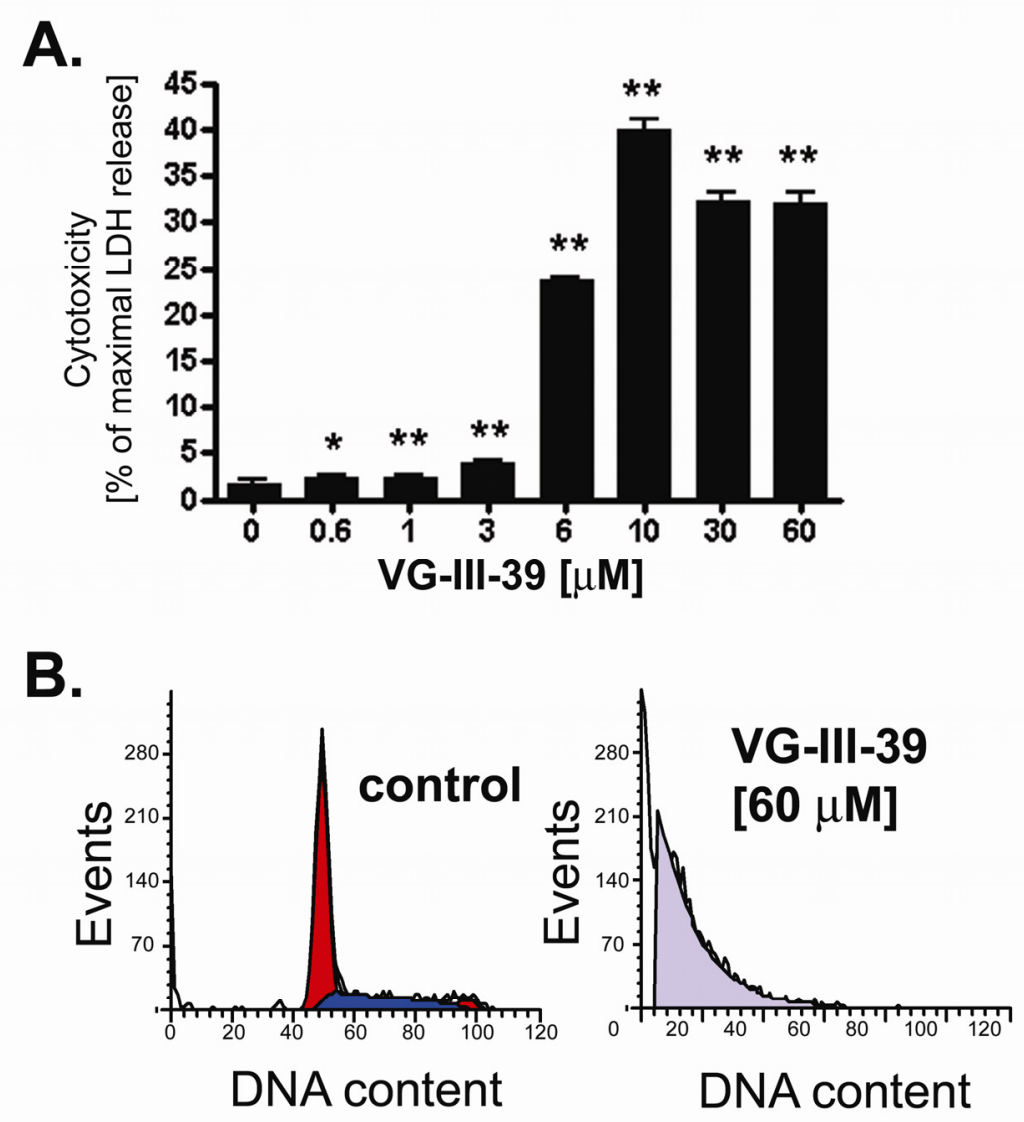

Figure 2-3. Compound VG-III-39 induced DNA degradation and LDH release.

SK-MEL-188 melanoma cells were incubated with VG-III-39 for 48 h. A: LDH released from the cells after treatment was then analyzed. Data is presented as mean $\pm \operatorname{SEM}(n=8)$, ${ }^{*} \mathrm{p}<0.05,{ }^{*} \mathrm{p}<0.001$. B: DNA content was analyzed using flow cytometry. Results are representative of two separate experiments. 
Table 2-2. Cytotoxic activity of thiazolidine analogs, Sorafenib and DTIC.

\begin{tabular}{|c|c|c|c|c|c|c|c|}
\hline \multirow{2}{*}{ Structure } & \multirow{2}{*}{ Compound } & \multirow{2}{*}{$\mathrm{R}$} & \multirow{2}{*}{$\mathrm{R}^{1}$} & \multirow{2}{*}{$\mathrm{R}^{2}$} & \multicolumn{3}{|c|}{$\mathrm{IC}_{50} \pm \operatorname{SEM}(\mu \mathrm{M})(\mathrm{n}=3)$} \\
\hline & & & & & SK-MEL-188 & WM-164 & $\mathrm{HDFa}$ \\
\hline & 1 & 3,4,5-trimethoxyl & $n-\mathrm{C}_{8} \mathrm{H}_{17}$ & $\mathrm{H}$ & $17 \pm 1$ & $20 \pm 1$ & $21 \pm 10$ \\
\hline & 2 & 3,4,5-trimethoxyl & $n-\mathrm{C}_{10} \mathrm{H}_{21}$ & $\mathrm{H}$ & $15 \pm 3$ & $2 \pm 0$ & $7 \pm 4$ \\
\hline & 3 & 3,4,5-trimethoxyl & $n-\mathrm{C}_{12} \mathrm{H}_{25}$ & $\mathrm{H}$ & $2 \pm 0$ & $2 \pm 0$ & $2 \pm 1$ \\
\hline & 4 & 3,4,5-trimethoxyl & $n-\mathrm{C}_{14} \mathrm{H}_{29}$ & $\mathrm{H}$ & $2 \pm 1$ & $2 \pm 0$ & $3 \pm 0$ \\
\hline & 5 & 3,4,5-trimethoxyl & $n-\mathrm{C}_{16} \mathrm{H}_{33}$ & $\mathrm{H}$ & $2 \pm 0$ & $1 \pm 0$ & $2 \pm 0$ \\
\hline & 6 & 3,4-dimethoxyl & $n-\mathrm{C}_{10} \mathrm{H}_{21}$ & $\mathrm{H}$ & $12 \pm 6$ & $6 \pm 3$ & $6 \pm 1$ \\
\hline & 7 & 3,4-dimethoxyl & $n-\mathrm{C}_{12} \mathrm{H}_{25}$ & $\mathrm{H}$ & $3 \pm 1$ & $2 \pm 1$ & $5 \pm 2$ \\
\hline & 8 & 3,4-dimethoxyl & $n-\mathrm{C}_{14} \mathrm{H}_{29}$ & $\mathrm{H}$ & $2 \pm 1$ & $1 \pm 0$ & $3 \pm 1$ \\
\hline & 9 & 3,4-dimethoxyl & $n-\mathrm{C}_{16} \mathrm{H}_{33}$ & $\mathrm{H}$ & $2 \pm 0$ & $1 \pm 0$ & $2 \pm 1$ \\
\hline & 10 & $3,4-\mathrm{OCH}_{2} \mathrm{O}-$ & $n-\mathrm{C}_{10} \mathrm{H}_{21}$ & $\mathrm{H}$ & $7 \pm 1$ & $5 \pm 0$ & $8 \pm 3$ \\
\hline & 11 & $3,4-\mathrm{OCH}_{2} \mathrm{O}-$ & $n-\mathrm{C}_{12} \mathrm{H}_{25}$ & $\mathrm{H}$ & $4 \pm 0$ & $3 \pm 0$ & $5 \pm 1$ \\
\hline & 12 & $3,4-\mathrm{OCH}_{2} \mathrm{O}-$ & $n-\mathrm{C}_{14} \mathrm{H}_{29}$ & $\mathrm{H}$ & $2 \pm 0$ & $1 \pm 0$ & $4 \pm 1$ \\
\hline & 13 & $3,4-\mathrm{OCH}_{2} \mathrm{O}-$ & $n-\mathrm{C}_{16} \mathrm{H}_{33}$ & $\mathrm{H}$ & $2 \pm 0$ & $2 \pm 0$ & $6 \pm 2$ \\
\hline & 14 & $\mathrm{NHCOCH}_{3}$ & $n-\mathrm{C}_{12} \mathrm{H}_{25}$ & $\mathrm{H}$ & $2 \pm 0$ & $1 \pm 0$ & $4 \pm 0$ \\
\hline & 15 & $\mathrm{NHCOCH}_{3}$ & $n-\mathrm{C}_{14} \mathrm{H}_{29}$ & $\mathrm{H}$ & $2 \pm 0$ & $1 \pm 0$ & $4 \pm 1$ \\
\hline & 16 & $\mathrm{NHCOCH}_{3}$ & $n-\mathrm{C}_{15} \mathrm{H}_{31}$ & $\mathrm{H}$ & $2 \pm 0$ & $1 \pm 0$ & $14 \pm 2$ \\
\hline & 17 & $\mathrm{NHCOCH}_{3}$ & $n-\mathrm{C}_{16} \mathrm{H}_{33}$ & $\mathbf{H}$ & $2 \pm 0$ & $1 \pm 0$ & $19 \pm 8$ \\
\hline & 18 & $\mathrm{NHCOCH}_{3}$ & $n-\mathrm{C}_{17} \mathrm{H}_{35}$ & $\mathrm{H}$ & $9 \pm 0$ & $2 \pm 0$ & $36 \pm 5$ \\
\hline & 19 & $\mathrm{NHCOCH}_{3}$ & $n-\mathrm{C}_{18} \mathrm{H}_{37}$ & $\mathrm{H}$ & $22 \pm 3$ & $12 \pm 1$ & $>60$ \\
\hline & $20 Z$ & $\mathrm{NHCOCH}_{3}$ & (Z)-Octadec-8-enyl & $\mathbf{H}$ & $1 \pm 0$ & $1 \pm 0$ & $11 \pm 1$ \\
\hline & $20 \mathrm{E}$ & $\mathrm{NHCOCH}_{3}$ & (E)-Octadec-8-enyl & $\mathbf{H}$ & $3 \pm 0$ & $1 \pm 0$ & $18 \pm 4$ \\
\hline & 21 & $\mathrm{H}$ & $n-\mathrm{C}_{14} \mathrm{H}_{29}$ & $\mathrm{H}$ & $2 \pm 1$ & $1 \pm 0$ & $3 \pm 0$ \\
\hline & 22 & $\mathrm{H}$ & $n-\mathrm{C}_{16} \mathrm{H}_{33}$ & $\mathrm{H}$ & $2 \pm 0$ & $1 \pm 0$ & $2 \pm 0$ \\
\hline & 23 & $\mathrm{H}$ & $\mathrm{OCH}_{3}$ & $\mathrm{CH}_{3}$ & $>100$ & $>100$ & $>100$ \\
\hline & 26 & $\mathrm{NH}_{2}$ & $n-\mathrm{C}_{12} \mathrm{H}_{25}$ & $\mathrm{H}$ & $2 \pm 0$ & $1 \pm 0$ & $4 \pm 1$ \\
\hline & 27 & $\mathrm{NH}_{2}$ & $n-\mathrm{C}_{16} \mathrm{H}_{33}$ & $\mathrm{H}$ & $2 \pm 0$ & $1 \pm 0$ & $7 \pm 1$ \\
\hline
\end{tabular}


Table 2-2. (continued)

\begin{tabular}{|c|c|c|c|c|c|c|c|}
\hline \multirow{2}{*}{ Structure } & \multirow{2}{*}{ Compound } & \multirow{2}{*}{$\mathrm{R}$} & \multirow{2}{*}{$\mathrm{R}^{1}$} & \multirow{2}{*}{$\mathrm{R}^{2}$} & \multicolumn{3}{|c|}{$\mathrm{IC}_{50} \pm \operatorname{SEM}(\mu \mathrm{M})(\mathrm{n}=3)$} \\
\hline & & & & & SK-MEL-188 & WM-164 & $\mathrm{HDFa}$ \\
\hline & 24 & 3,4,5-trimethoxyl & $n-\mathrm{C}_{14} \mathrm{H}_{29}$ & $\mathrm{H}$ & $2 \pm 0$ & $1 \pm 0$ & $4 \pm 1$ \\
\hline & 25 & $\mathrm{NHCOCH}_{3}$ & $n-\mathrm{C}_{16} \mathrm{H}_{33}$ & $\mathrm{H}$ & $3 \pm 0$ & $1 \pm 0$ & $23 \pm 3$ \\
\hline & 28 & $\mathrm{H}$ & $n-\mathrm{C}_{14} \mathrm{H}_{29}$ & $\mathrm{H}$ & $42 \pm 5$ & $>50$ & $>50$ \\
\hline & 29 & 3,4-dimethoxyl & $n-\mathrm{C}_{14} \mathrm{H}_{29}$ & $\mathrm{H}$ & $19 \pm 2$ & $43 \pm 10$ & $>50$ \\
\hline & 30 & $\mathrm{NHCOCH}_{3}$ & $n-\mathrm{C}_{16} \mathrm{H}_{33}$ & $\mathrm{H}$ & $29 \pm 1$ & $>100$ & $>100$ \\
\hline & 31 & 3,4,5-trimethoxyl & $\mathrm{N} / \mathrm{A}$ & $\mathrm{N} / \mathrm{A}$ & $>50$ & $>50$ & $>50$ \\
\hline & DTIC & & & & $>100$ & $>100$ & $>100$ \\
\hline & Sorafenib & & & & $5 \pm 1$ & $5 \pm 0$ & $15 \pm 1$ \\
\hline
\end{tabular}

NA: not available. 
and composition of the side chain are critical for their activity. There is no significant difference in their activity between the cis- and trans- isomers.

Removing the acetyl amino group on the phenyl ring (compound 21 and 22) resulted in the loss of selectivity, although potency was similar to those with this substitution (compounds 15 and 17). Replacing the alkyl chain with a methoxyl group completely abolished potency (compound 23). Changing the chirality from an $R$ to $S$ configuration at the $\mathrm{C} 4$ position on the thiazolidine ring did not substantially affect either potency or selectivity (compound 4 vs. 24 and compound 17 vs. 25). Selectivity has a strong dependence on the substitutions in the phenyl ring. For example, with a $\mathrm{C} 12$ chain, potency is similar for all the substitutions I studied (compounds 3, 7, 11, 14, and 26). However, selectivity improves dramatically when proper substitutions are present (compound $17 v s$. compounds 5, 9, 13, 22, and 27).

When the amino group in the thiazolidine ring is substituted (compound 30) or a double bond is introduced (compounds 28 and 29), the resulting compounds are largely inactive with $\mathrm{IC}_{50}$ values above $20 \mu \mathrm{M}$. I also tested the intermediate compound in which the amino group is protected by a Boc group, and that compound is inactive also (data not shown). Furthermore, when Dr Yan Lu removed the aliphatic chain and the amino group by synthesizing a dimer, she obtained an inactive compound (compound 31). These results clearly demonstrate the importance of the amino group in the thiazolidine ring.

\subsubsection{Further SAR Studies with New Thiazolidine Derivatives}

The diastereomeric mixtures of compounds 3aa-15c were used to evaluate their in vitro inhibitory activity and toxicity against two human melanoma cell lines (A375 and WM-164), one mouse melanoma cell line (B16-F1), fibroblast cells (control cell line for melanoma). Because preparing pure diastereomers was not easy to achieve, the $\mathrm{IC}_{50}$ values were obtained on diastereomeric mixtures to select the most promising compounds. The standard sulforhodamine B (SRB) assay was used to evaluate the antiproliferative activity of different compounds in melanoma cells. The results are summarized in Table 2-3. Compound $1 \mathrm{a}$ and $1 \mathrm{~b}$ were chosen as control compounds for both tumor cell lines in these in vitro studies. Cisplatin (CDDP), DTIC, and Sorafenib (Nexavar, Bay43-9006, which had been granted Fast Track designation by FDA to treat advanced melanoma) were used as reference compounds for melanoma (97). The antiproliferative data showed that all three melanoma cell lines were resistant to cisplatin. DTIC was inactive because of the lack of bioactivation in vitro $(98,99)$. The average $\mathrm{IC}_{50}$ value of Sorafenib on three melanoma cell lines is $5 \mu \mathrm{M}$, and the selectivity of Sorafenib between fibroblast cells and melanoma cells is 3-fold. Our purpose in modifying chemical structure was to enhance the selectivity between tumor cells and control cells and to increase or maintain antitumor potency. Thus, I used the average $\mathrm{IC}_{50}$ value of all melanoma and prostate cancer cells for comparison. Selectivity is defined as the ratio of $\mathrm{IC}_{50}$ values in the control cell line and the average in tumor cell lines. From Tables 2.3, the most selective compound ( $3 \mathrm{id}$ ) had a selectivity of 11.3-fold in melanoma cells against fibroblast cells. 
Table 2-3. Cytotoxic activity of ATCAA on three melanoma cell lines and a fibroblast cell line.

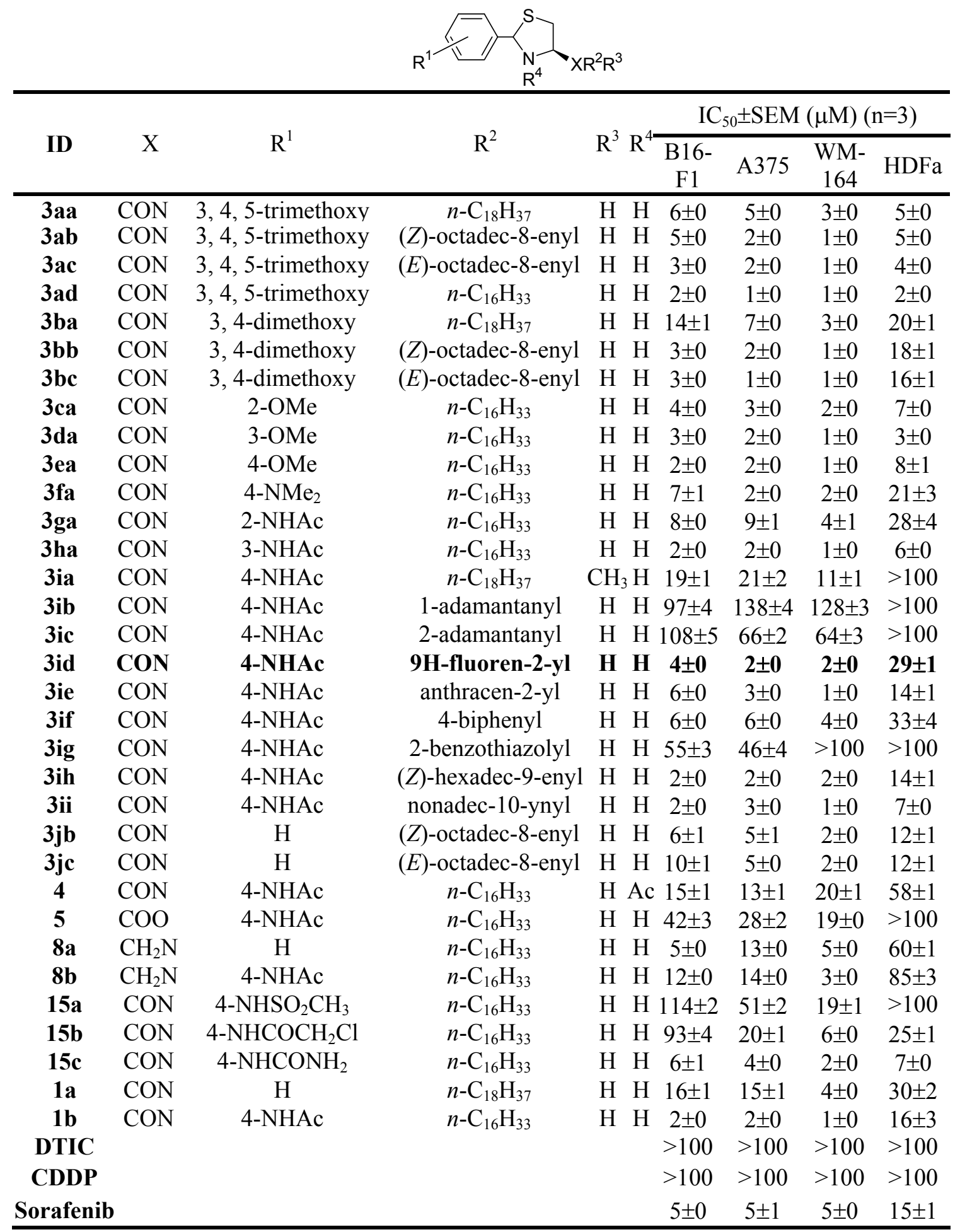




\subsubsection{SAR of Different Amide Chains and Chain Length of ATCAA}

ATCAA molecules were designed from LPA structure, which contains a long aliphatic chain. To examine whether the fatty chain played an essential role in cytotoxicity, we explored the possibilities of mimic fatty chain with bulky ring or aromatic systems such as adamantanyl, fluorenyl, and anthrancenyl. Although the antiproliferative data showed that 1- or 2-adamantanyl amides and 2-benzothiazolylamide (3ib, 3ic, and 3ig) had lower activity against most melanoma cells, introducing certain aromatic bulky groups such as 9H-fluoren-2-yl (3id), anthrancen-2-yl (3ie), and 4biphenyl (3if) retained their proliferative activity. Of particular interest, while introducing 9-fluoren-2-yl (3id) showed a comparable $\mathrm{IC}_{50}$ value of $3 \mu \mathrm{M}$ with $1 \mathrm{~b}(2 \mu \mathrm{M})$ on melanoma cells, the selectivity of 3id increased to 11 fold from 9 fold in $1 \mathrm{~b}$. This structural modification provided us a new approach to optimize the drug-like properties of ATCAA molecules, in which the hydrophobic fatty lipid chain could be modified with other groups (100).

Hexadecyl chains ( $3 \mathrm{ad})$ displayed both the highest potency and selectivity against cancer cells among the examined chains, with an $\mathrm{IC}_{50}=1 \mu \mathrm{M}$ for WM-164 cells. However, further increasing chain length (18 carbons, $3 \mathrm{aa}$ ) reduced potency and toxicity. Interestingly, adding either a cis- or trans- double bond in the $\mathrm{C} 18$ side chain restored potency dramatically (cis- and trans-octadec-8-enyl), demonstrating that both length and composition of the side chain are critical for their activity. Trans-octadec-8-enyl chain (3ac, 3bc, 3jc) showed slightly better activity than did the cis-isomers (3ab, 3bb, 3jb) against melanoma cells. Introducing a cis-double bond in the C16 fatty chain (3ih) or an alkyne in the $\mathrm{C} 18$ chain (3ii) did not increase overall potency. Introducing a branched aliphatic chain (3ia) decreased the potency in both melanoma cells (average $\mathrm{IC}_{50}=17 \mu \mathrm{M}$, 9.3 fold decrease) compared with $1 \mathrm{~b}$ (average $\mathrm{IC}_{50}=2 \mu \mathrm{M}$ ).

\subsubsection{SAR of Substitutions on 2-Phenyl of ATCAA}

The antiproliferative effects indicated that when methoxy, 3, 4-dimethoxy, and 3, 4, 5-trimethoxy were introduced to 2-phenyl (3aa-3ea), potent activity was preserved or increased compared with that of 1a. For example, (2RS, 4R)-2-(3, 4, 5-trimethoxyphenyl) thiazolidine-4-carboxylic acid octadecylamide (3aa) (average $\mathrm{IC}_{50}=5 \mu \mathrm{M}$ ) was 3 fold more potent than was 1a (average $\mathrm{IC}_{50}=12 \mu \mathrm{M}$ ). Subsequently, different groups were introduced to the para-phenyl position. Replacements of 4- $\mathrm{NHCOCH}_{3}(1 \mathrm{~b})$ with 4-N $\left(\mathrm{CH}_{3}\right)_{2}$ (3fa) showed comparable activity on $\mathrm{A} 375\left(\mathrm{IC}_{50}=2\right.$ vs. $\left.2 \mu \mathrm{M}\right)$ and WM-164 cells $\left(\mathrm{IC}_{50}=2 v s .1 \mu \mathrm{M}\right)$ but slightly lower selectivity on fibroblast cells $\left(\mathrm{IC}_{50}=21 v s .16 \mu \mathrm{M}\right)$.

Although 4-N $\left(\mathrm{CH}_{3}\right)_{2}$ (3fa) substituted ATCAAs were more potent than was 1a (average $\mathrm{IC}_{50}=3 \mu \mathrm{M}$ for $3 \mathrm{fa}$, and $12 \mu \mathrm{M}$ for $1 \mathrm{a}$ ), they were less potent than $1 \mathrm{~b}$ (average $\mathrm{IC}_{50}=2 \mu \mathrm{M}$ and selective ratio $=8.9$ for $1 \mathrm{~b}$ ). Methanesulfonamide $(15 \mathrm{a})$ or 2 chloroacetamide $(15 \mathrm{~b})$ were less potent $\left(\mathrm{IC}_{50}=6 \sim 114 \mu \mathrm{M}\right)$ and less toxic $\left(\mathrm{IC}_{50}=25 \sim 100\right.$ $\mu \mathrm{M})$ on melanoma cell lines compared with $1 \mathrm{~b}$. Introducing an ureido, 4- $\mathrm{NHCONH}_{2}$ 
(15c) instead of a 4- $\mathrm{NHCOCH}_{3}$ kept partial potency (average $\mathrm{IC}_{50}=4 \mu \mathrm{M}$ ) but resulted in loss of selectivity against melanoma cells (1.8-fold for $15 \mathrm{c} v$ s. 8.9 -fold for $1 \mathrm{~b})$.

We also investigated the importance of $o-, m$-, and $p$ - substitutions in the 2-phenyl ring relative to acetylamino and methoxy groups. Interestingly, $o-, m-$, and $p$ - isomers showed different activities. $P$-Isomers $(3 \mathrm{ea}, 1 \mathrm{~b})$ had the best selectivity and activity. $M$ Isomers (3da, 3ha) had similar average $\mathrm{IC}_{50} \mathrm{~S}$ against melanoma compared with $p$ isomers, but their toxicity also increased on fibroblast $(3 \mu \mathrm{M}) . o-\mathrm{MeO}$ and $o$-NHAc analogues (3ca, 3ga) showed slightly less potency and selectivity.

\subsubsection{SAR of 4-Linkage of ATCAA and Thiazolidine Ring}

To investigate the importance of amide linkage on C4 of ATCAA, the carboxamide was replaced with an ester and amine. The isosteric replacement of the amide by an ester resulted in 5 with markedly decreased activity against all cancer cell lines. The amine derivatives $8 \mathrm{a}$ and $8 \mathrm{~b}$ also failed to show any increased potency, although they did show lower toxicity $(60-85 \mu \mathrm{M})$ than did $1 \mathrm{a}(30 \mu \mathrm{M})$ and $1 \mathrm{~b}(16 \mu \mathrm{M})$ in fibroblast cells.

Thiazolidine-4-carboxylic acid has been reported to be effective for treating advanced cancers $(101,102)$. To examine the importance of a central thiazolidine ring, Dr Yan Lu synthesized 3-acetyl-thiazolidine compound 4 and a series of thiazolidine ring-opened compounds 16a-16d. The structure of these ring-open and their relationship is shown if Figure 2-4. Antiproliferative activity of 16a-16d against melanoma is summarized in Table 2-4. When acetyl was introduced to 3-NH of thiazolidine, activity decreased in both cancer cell lines (average $\mathrm{IC}_{50}=16$ and $10 \mu \mathrm{M}$ against melanoma and prostate cancer, respectively). Cleavage of both the C-S and C-N bond (16a) led to substantial decrease in both potency (13 14 $\mu \mathrm{M}$ on melanoma cell lines) and toxicity (61 $\mu \mathrm{M}$ on the control cells) compared with $1 \mathrm{~b}$ and $3 \mathrm{ja}(1 \sim 2 \mu \mathrm{M}$ on cancer cells, $2 \sim 16 \mu \mathrm{M}$ on control cells). Opening the thiazolidine ring from the C-S bond (16b) caused a loss of potency on both cancer and control cells $(>100 \mu \mathrm{M})$. Cleavage of the $\mathrm{C}-\mathrm{N}$ bond in the thiazolidine ring (16c) also resulted in decreased activity $(13 \sim 15 \mu \mathrm{M})$ and no improvement on selectivity compared with $3 \mathrm{ja}$. Removing the C-S bond (16d) reduced the activity of $1 \mathrm{~b}$ from $\mathrm{IC}_{50}=1 \sim 2 \mu \mathrm{M}$ to $10 \sim 12 \mu \mathrm{M}$. These results suggested that the presence of the thiazolidine ring is critical for activity. Attempts to reduce the amide with LAH or $\mathrm{B}_{2} \mathrm{H}_{6}$ resulted in the C-S bond cleavage in thiazolidine. A disulfanyl dimer, which completely lost anticancer activity, was obtained, and this result is consistent with the above finding that the thiazolidine ring played an essential role in ATCAA potency. 


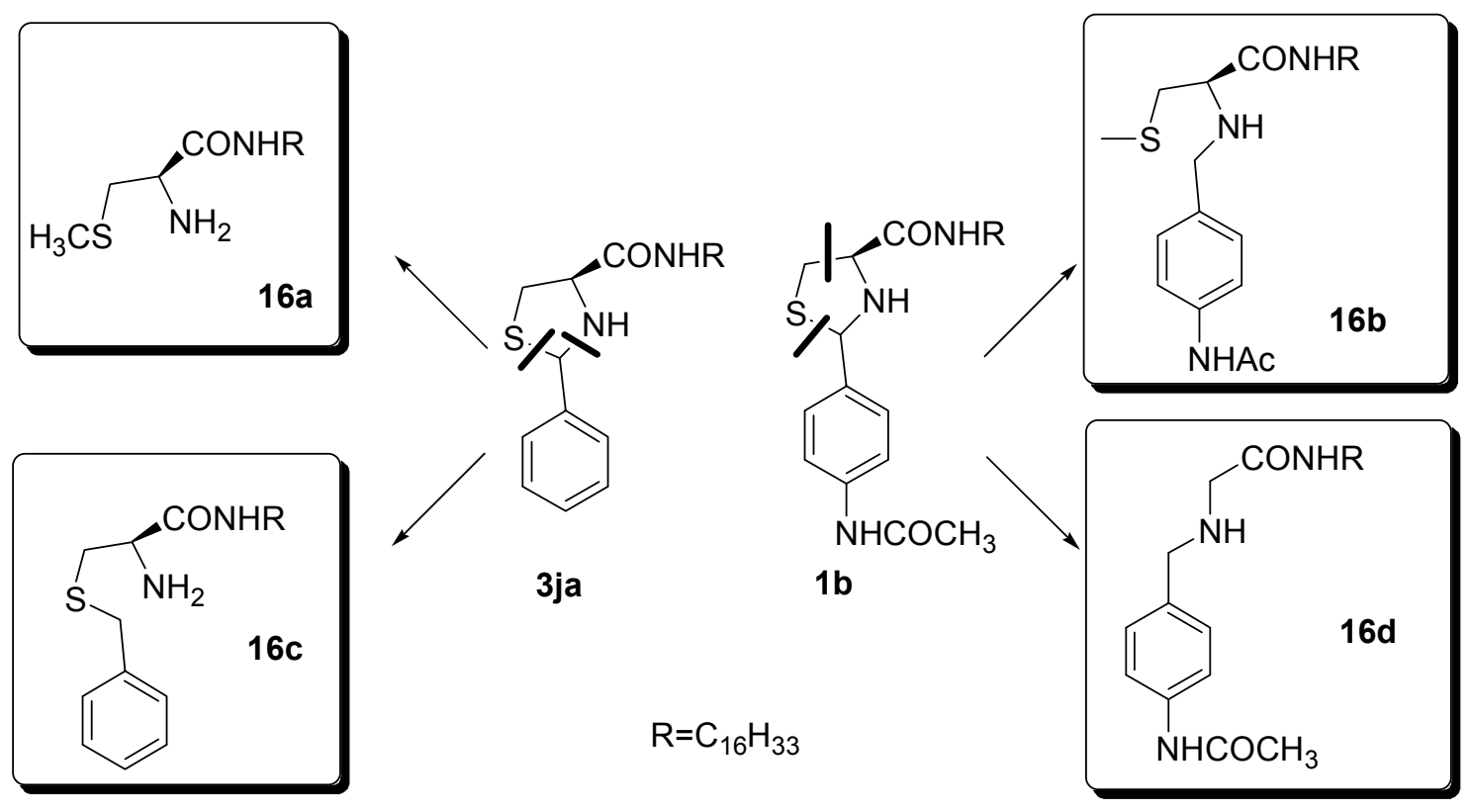

Figure 2-4. Structure relationship of thiazolidine ring-open compounds.

Table 2-4. Cytotoxic activity of thiazolidine ring-open compounds against melanoma.

\begin{tabular}{cccc}
\hline \multirow{2}{*}{ ID } & \multicolumn{3}{c}{$\mathrm{IC}_{50} \pm \mathrm{SEM}(\mu \mathrm{M})(\mathrm{n}=3)$} \\
\cline { 2 - 4 } & $\mathrm{A} 375$ & $\mathrm{WM}-164$ & $\mathrm{HDFa}$ \\
\hline $\mathbf{1 6 a}$ & $13 \pm 1$ & $14 \pm 1$ & $61 \pm 4$ \\
$\mathbf{1 6 b}$ & $109 \pm 5$ & $145 \pm 5$ & $>100$ \\
$\mathbf{1 6 c}$ & $15 \pm 1$ & $13 \pm 2$ & $16 \pm 1$ \\
$\mathbf{1 6 d}$ & $12 \pm 1$ & $11 \pm 1$ & $24 \pm 1$ \\
3ja & $\mathrm{ND}$ & $1 \pm 0$ & $2 \pm 0$ \\
$\mathbf{1 b}$ & $2 \pm 0$ & $1 \pm 0$ & $16 \pm 3$ \\
\hline
\end{tabular}

ND: not determined 


\subsubsection{SAR Summary of ATCAA for Melanoma}

After extensive chemical structure modification and in vitro activity studies on melanoma cells, the overall SAR of ATCAA compounds on melanoma cells was summarized in Figure 2-5. From the above studies, we generalized several important SAR. Firstly, Thiazolidine ring and the free NH on the ring are very critical for the antimelanoma activity. The carbon 4 chiral center on the thiazolidine ring is not important for activity because both isomers showed similar potency. Secondly, for the side chain, aliphatic chain with a length of 16 carbons was best for activity. It also can be replaced by $9 \mathrm{H}$-fluoren-2-yl group. Thirdly, the link between the thiazolidine ring and the side chain prefer amide to ester and amine. Lastly, for the substituents on the phenyl ring, electron donating group (EDG) is better than electron withdrawing group (EWG), paraand meta- substitution are the same, ortho-substitution is slightly worse.

\subsubsection{SAR of Newly Synthesized Imidazole and Imidazoline Analogs}

I found that the chirality at position 4 in thiazolidine ring of ATCAA analogs is not critical for their activity. However, the importance of chirality at position 2 is unknown, because all our previous compounds were tested as diastereomers. Separation of these stereoisomers by chromatographic methods turned out to be extremely difficult. Dr. Li asked for assistance from scientists from Chiralcel Technologies who tried both normal-phase and reverse-phase conditions with their nine chiral columns, but none of the combinations could separate these stereoisomers satisfactorily.

a. Free $\mathrm{NH}$ and thiazolidine ring contribute to anticancer activity; $4 R$ and $4 S$ isomers have similar potency

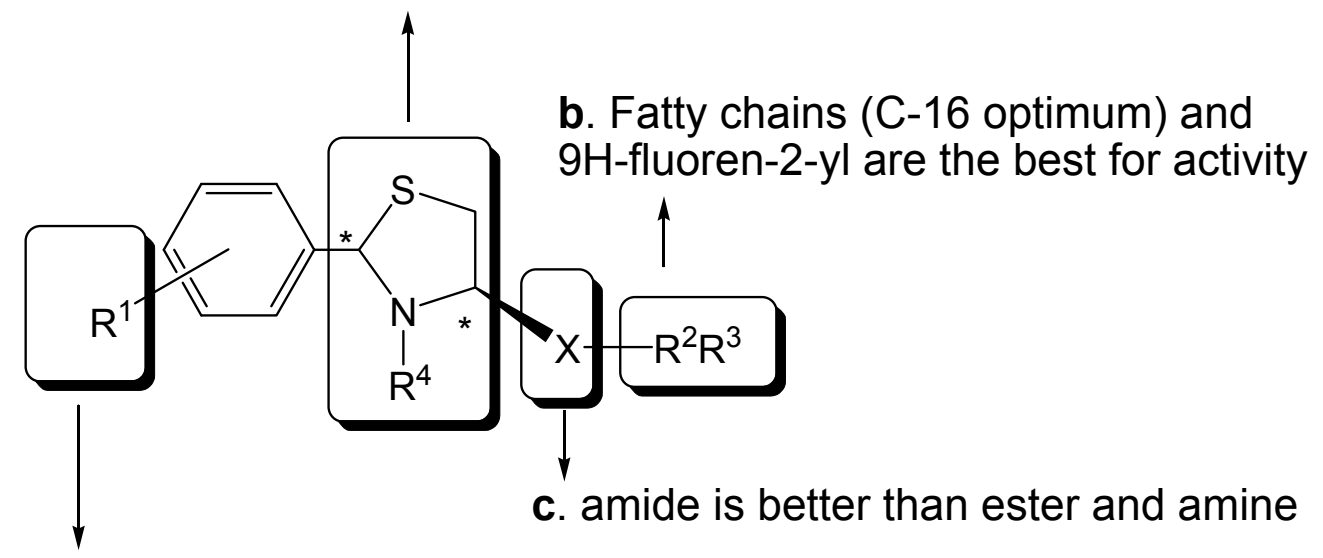

d. Phenyl substitutions: EDG is better than EWG; para- and metasubstituents are comparable; ortho-substituents are slightly worse

Figure 2-5. SAR summary of ATCAA for melanoma. 
Converting the thiazolidine ring to an imidazoline or imidazole ring represents a very productive approach. First, the nitrogen-containing ring would be very stable. Imidazoline and imidazole rings are important biological building blocks and are present in many existing drugs (103). Second, the imidazoline ring contains only one chiral center, and the imidazole ring contains no chiral center at all. These attributes may alleviate or even eliminate the need for future chiral separations. So Mr. Jianjun Chen made the following modification to ATCAA: a) replacing sulfur with nitrogen or carbon to understand the importance of the heteroatom in the ring, $b$ ) introducing one or two double bonds in the ring (imidazoline and imidazole) to understand the importance of the chiral centers, and c) introducing various spacers between the five-member ring and the aromatic ring to understand the relative spatial arrangements for the two rings.

I examined the antiproliferative activity of these imidazoline and imidazole analogs in two melanoma cell lines (human A375 cells and mouse B16 cells) and in a fibroblast cell line. The ability of these new analogs to inhibit the growth of melanoma cancer cell lines and fibroblast cells is summarized in Table 2-5.

In summary, none of the new analogs is as active as the lead compound. Although they do not contain any chiral centers, analogs containing an imidazole ring (compounds 5-11) did not have activity at concentrations below $10 \mu \mathrm{M}$. Replacing the imidazole ring with an imidazoline ring slightly improved the activity in general (compounds 15-19), with substantial improvement when trimethoxyl or amino acetyl substitutions were added (compounds 20-21). The structure activity for the side chain was similar to that of the thiazolidine analogs, with a saturated $\mathrm{C} 14 \sim \mathrm{C} 16$ chain or an unsaturated $\mathrm{C} 18$ chain to be optimal for activity against melanoma cells. Similarly, electronic donating substitutions on the paraposition of the aromatic ring increased activity (compounds 20-21). Replacing sulfur with carbon in the five-member ring produced an inactive compound (compound 30). Collectively, these results indicate that the thiazolidine ring is critical for the activity of this series of compounds.

When a spacer was inserted between the imidazoline/thiazolidine ring and the phenyl ring (compounds 22-29), there is no noticeable improvement in their activity (compound $20 v s$. compound 22). Increasing the size of the spacer by replacing one hydrogen in the methylene group with a methyl group reduced the activity (compound 23 vs. 26, and compound $24 v s .27$ ). Both of these results seemed to indicate detrimental effects of spacers for the activity. Finally, replacing the side chain with a fluorene group resulted in complete loss of activity (compound 23 vs. 25). However, with proper parasubstitution on the aromatic ring, the activity dramatically improved (compound 25 vs. compound 29) for this series of compounds. On the other hand, with a C16 side chain, the substitution on the phenyl ring had the opposite effect (compound 23 vs. 26) in sharp contrast to the non-chain analogs such as 29 and may indicate a different mechanism of action when the long side chain is replaced by a conjugated ring system. 
Table 2-5. Cytotoxic activity of imidazolines, imidazoles and comparison with Sorafenib and the lead thiazolidine compounds.

\begin{tabular}{|c|c|c|c|c|c|c|c|}
\hline \multirow{2}{*}{ Structure } & \multirow{2}{*}{ Compound } & \multirow{2}{*}{$\mathrm{R}$} & \multirow{2}{*}{$\mathrm{R}^{1}$} & \multirow{2}{*}{$\mathrm{R}^{2}$} & \multicolumn{3}{|c|}{$\mathrm{IC}_{50} \pm \operatorname{SEM}(\mu \mathrm{M})(\mathrm{n}=3)$} \\
\hline & & & & & A375 & $\mathrm{B} 16-\mathrm{F} 1$ & $\mathrm{HDFa}$ \\
\hline & 5 & $\mathrm{H}$ & $n-\mathrm{C}_{10} \mathrm{H}_{21}$ & $\mathrm{H}$ & $>30$ & $>30$ & $>30$ \\
\hline & 6 & $\mathrm{H}$ & $n-\mathrm{C}_{12} \mathrm{H}_{25}$ & $\mathrm{H}$ & $>30$ & $\sim 30$ & $>30$ \\
\hline & 7 & $\mathrm{H}$ & $n-\mathrm{C}_{14} \mathrm{H}_{29}$ & $\mathrm{H}$ & $19 \pm 1$ & $38 \pm 2$ & $43 \pm 2$ \\
\hline & 8 & $\mathrm{H}$ & $n-\mathrm{C}_{16} \mathrm{H}_{33}$ & $\mathrm{H}$ & $17 \pm 1$ & $40 \pm 2$ & $28 \pm 1$ \\
\hline & 9 & $\mathrm{H}$ & $n-\mathrm{C}_{18} \mathrm{H}_{37}$ & $\mathrm{H}$ & $21 \pm 1$ & $>30$ & $47 \pm 1$ \\
\hline & 10 & $\mathrm{H}$ & (E)-Octadec-8-enyl & $\mathrm{H}$ & $16 \pm 1$ & $32 \pm 1$ & $24 \pm 1$ \\
\hline & 11 & $\mathrm{H}$ & $p$-Bromophenyl & $\mathrm{H}$ & $>30$ & $>30$ & $>30$ \\
\hline & 15 & $\mathrm{H}$ & $n-\mathrm{C}_{14} \mathrm{H}_{29}$ & $\mathrm{H}$ & $7 \pm 0$ & $7 \pm 0$ & $17 \pm 2$ \\
\hline & 16 & $\mathrm{H}$ & $n-\mathrm{C}_{16} \mathrm{H}_{33}$ & $\mathrm{H}$ & $10 \pm 0$ & $6 \pm 0$ & $60 \pm 1$ \\
\hline & 17 & $\mathrm{H}$ & $n-\mathrm{C}_{18} \mathrm{H}_{37}$ & $\mathrm{H}$ & $54 \pm 1$ & $51 \pm 1$ & $111 \pm 4$ \\
\hline & 18 & $\mathrm{H}$ & (E)-Octadec-8-enyl & $\mathrm{H}$ & $8 \pm 0$ & $12 \pm 0$ & $25 \pm 3$ \\
\hline & 19 & 3,4,5-trimethoxyl & $\mathrm{OCH}_{3}$ & $\mathrm{CH}_{3}$ & $>20$ & $>20$ & $>20$ \\
\hline & 20 & 3,4,5-trimethoxyl & $n-\mathrm{C}_{16} \mathrm{H}_{33}$ & $\mathrm{H}$ & $4 \pm 1$ & $4 \pm 0$ & $13 \pm 1$ \\
\hline & 21 & $p-\mathrm{NHCOCH}_{3}$ & $n-\mathrm{C}_{16} \mathrm{H}_{33}$ & $\mathrm{H}$ & $5 \pm 0$ & $2 \pm 0$ & $15 \pm 2$ \\
\hline & 22 & 3,4,5-trimethoxyl & $n-\mathrm{C}_{16} \mathrm{H}_{33}$ & $\mathrm{H}$ & $4 \pm 0$ & $4 \pm 0$ & $16 \pm 2$ \\
\hline & 23 & $\mathrm{H}$ & $n-\mathrm{C}_{16} \mathrm{H}_{33}$ & $\mathrm{H}$ & $8 \pm 1$ & $7 \pm 0$ & $15 \pm 1$ \\
\hline & 24 & $\mathrm{H}$ & (E)-Octadec-8-enyl & $\mathrm{H}$ & $10 \pm 1$ & $12 \pm 0$ & $16 \pm 2$ \\
\hline & 25 & $\mathrm{H}$ & 2-Fluorene & $\mathrm{H}$ & $174 \pm 11$ & $118 \pm 8$ & $>100$ \\
\hline & 26 & $\mathrm{H}$ & $n-\mathrm{C}_{16} \mathrm{H}_{33}$ & $\mathrm{CH}_{3}$ & $13 \pm 1$ & $12 \pm 0$ & $56 \pm 7$ \\
\hline & 27 & $\mathrm{H}$ & (E)-Octadec-8-enyl & $\mathrm{CH}_{3}$ & $21 \pm 1$ & $17 \pm 1$ & $237 \pm 39$ \\
\hline & 28 & $p-\mathrm{NHCOCH}_{3}$ & $n-\mathrm{C}_{16} \mathrm{H}_{33}$ & $\mathrm{H}$ & $191 \pm 14$ & $171 \pm 21$ & $>100$ \\
\hline & 29 & & & & $14 \pm 0$ & $12 \pm 0$ & $47 \pm 2$ \\
\hline & Sorafenib & & & & $5 \pm 1$ & $5 \pm 0$ & $15 \pm 1$ \\
\hline
\end{tabular}




\subsubsection{Flow Cytometry Analysis of Active ATCAA Compounds}

Flow cytometry analysis was performed on human melanoma A375 to examine the effect of the new synthetics on cell cycle progression. Analysis of the DNA content of control cells (Figure 2-6, top graphs) showed typical distribution of peaks corresponding to cells in G1/G0 phase (65.67 66.97\%), S-phase (24.16 28.36\%), and G2/M-phase (5.97 8.87\%). Upon treatment with compounds $3 \mathrm{ad}$ and $1 \mathrm{~b}$ at 1, 5, 10, and $20 \mu \mathrm{M}$ concentrations for $48 \mathrm{~h}$, flow cytometric analysis showed that these compounds significantly induced apoptosis (sub-G1 phase) in cancer cells in a dose-dependent manner. The accumulation of cells in sub-G1 phase became apparent at $5 \mu \mathrm{M}$.

\subsubsection{ATCAA Inhibited Human Melanoma Colony Formation in Soft Agar}

Cells grown in 96-well plates do not reflect many properties associated with three-dimensional tumors. Human tumor colony formation assay (ex vivo soft agar colony assay) has been suggested as an in vitro method to predict the response of an individual patient's tumor to chemotherapeutic agents $(104,105)$. As a prelude to in vivo animal experiments, I investigated the ability of compound $1 \mathrm{~b}$ and $3 \mathrm{ad}$ to inhibit melanoma colony formation by using a well-established method $(93,106,107)$. I performed studies with A375 human melanoma cells. Photos and quantified results are shown in Figure 2-7 and Figure 2-8. Compounds $1 \mathrm{~b}$ and $3 \mathrm{ad}$ effectively inhibited A375 colony formation at 2 $\mu \mathrm{M}$, the lowest tested concentration. At a concentration of $20 \mu \mathrm{M}$, compound 3 ad almost completely inhibited colony formation, while compound $1 \mathrm{~b}$ inhibited colony formation by $75 \%$. At the highest tested concentration, $100 \mu \mathrm{M}$, melanoma colony formation was completely inhibited by both $1 \mathrm{~b}$ and $3 \mathrm{ad}$.

\subsubsection{Antitumor Efficacy of ATCAA on A375 Xenograft Model}

I chose human melanoma A375 cells to test efficacy in vivo because ATCAA exhibited potency both in vitro assay and colony formation assay against A375 cells. A375 xenograft tumors also exhibited faster tumor growth rate. While compound 3ad was more potent than was $1 \mathrm{~b}$ in inhibiting melanoma colony formation, it had much poorer selectivity between melanoma cancer cells and fibroblast cells (2.0 fold for $3 \mathrm{ad} v s$. 8.9 fold for $1 b$ ) as shown in Table 2-3. Therefore, I chose $1 \mathrm{~b}$ for our in vivo studies against A375 melanoma tumors. This compound was formulated with $80 \%$ Tween 80 and $20 \%$ Captex 200 because of its limited water solubility (108). I first determined the maximally tolerated dose (MTD) in ICR (imprinting control region) mice. These mice are less expensive than nude mice and are commonly used for toxicity testing. The MTD for compound $1 \mathrm{~b}$ was determined to be $42 \mathrm{mg} / \mathrm{kg}$. Two nontoxic dosages ( $5 \mathrm{mg} / \mathrm{kg}$ and 10 $\mathrm{mg} / \mathrm{kg})$ were used. I also included DTIC $(60 \mathrm{mg} / \mathrm{kg})$ the gold standard for melanoma treatment, as a positive control to assess the efficacy of compound 1b (109). Briefly, male athymic nude mice were injected subcutaneously with $2.5 \times 10^{6} \mathrm{~A} 375$ cells. Treatment began on day 7 after tumor inoculation, and $1 \mathrm{~b}$ was injected once a day. Each group had 

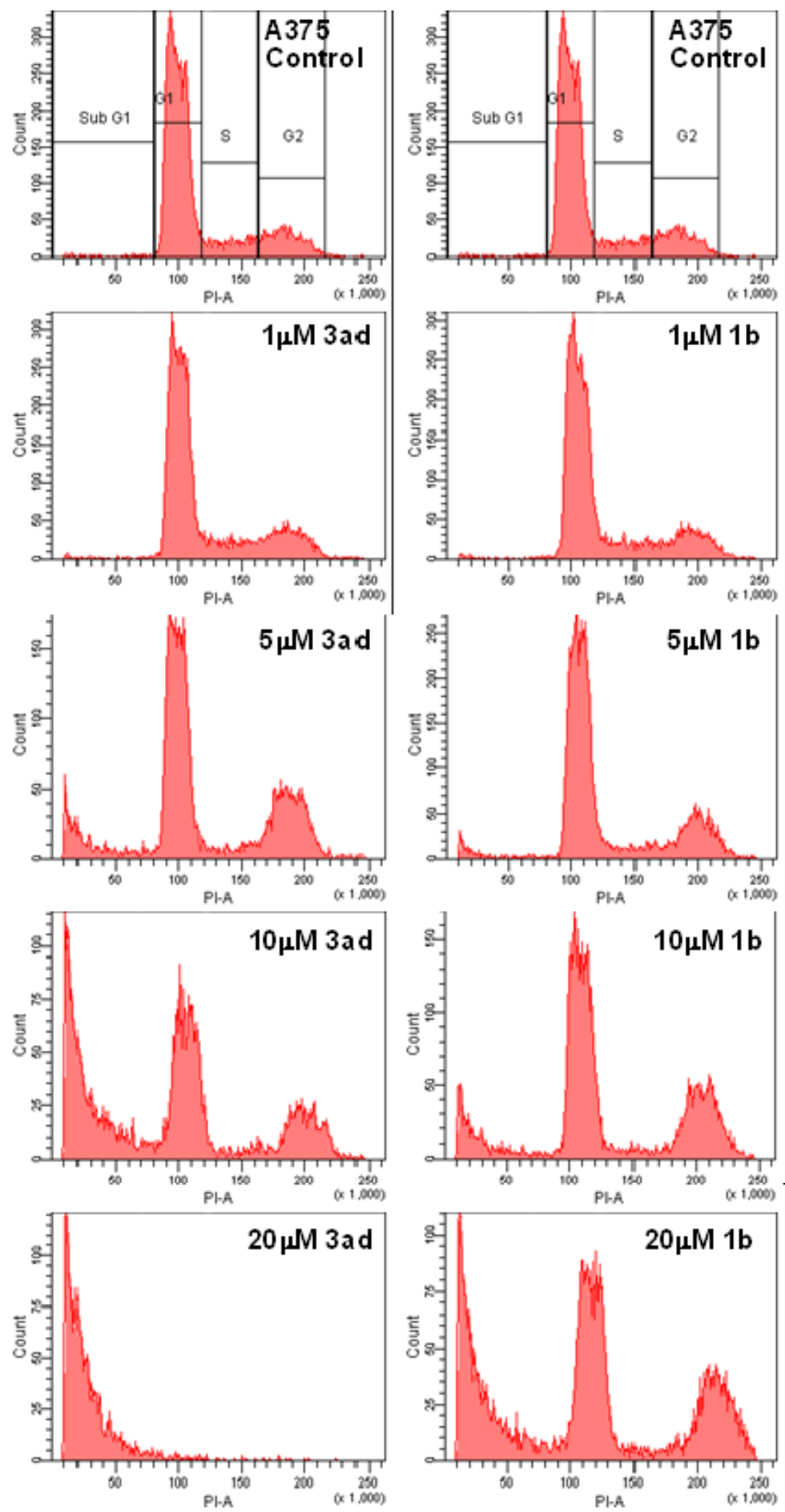

Figure 2-6. Flow cytometry analysis of selected ATCAA compounds.

Sub-G1 phase accumulation was induced by tested compounds on melanoma A375 cells. (This experiment was done by Mr. Chien-Ming Li, Division of Pharmaceutics, College of Pharmacy, the Ohio State University). 

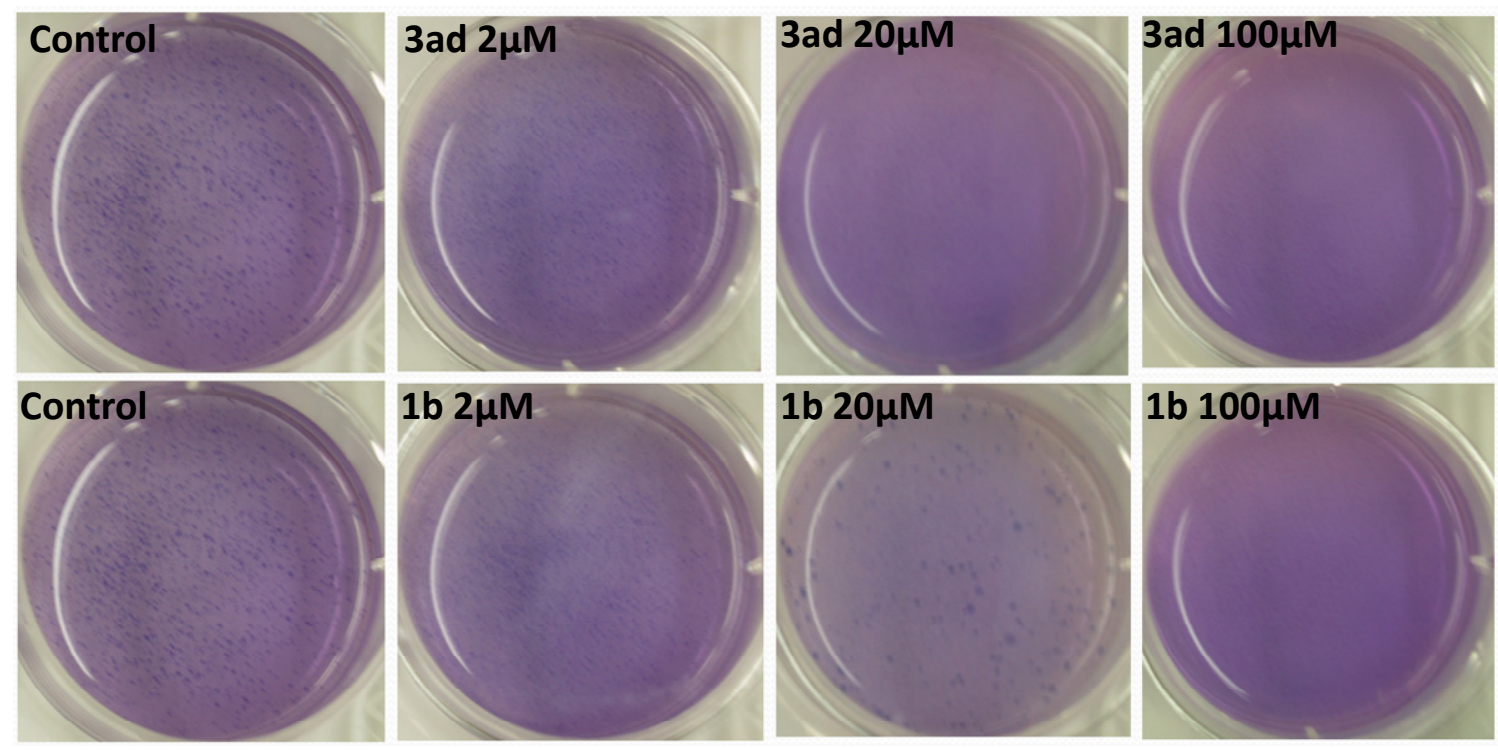

Figure 2-7. Human melanoma A375 cells colony formation in soft agar with ATCAA treatment.
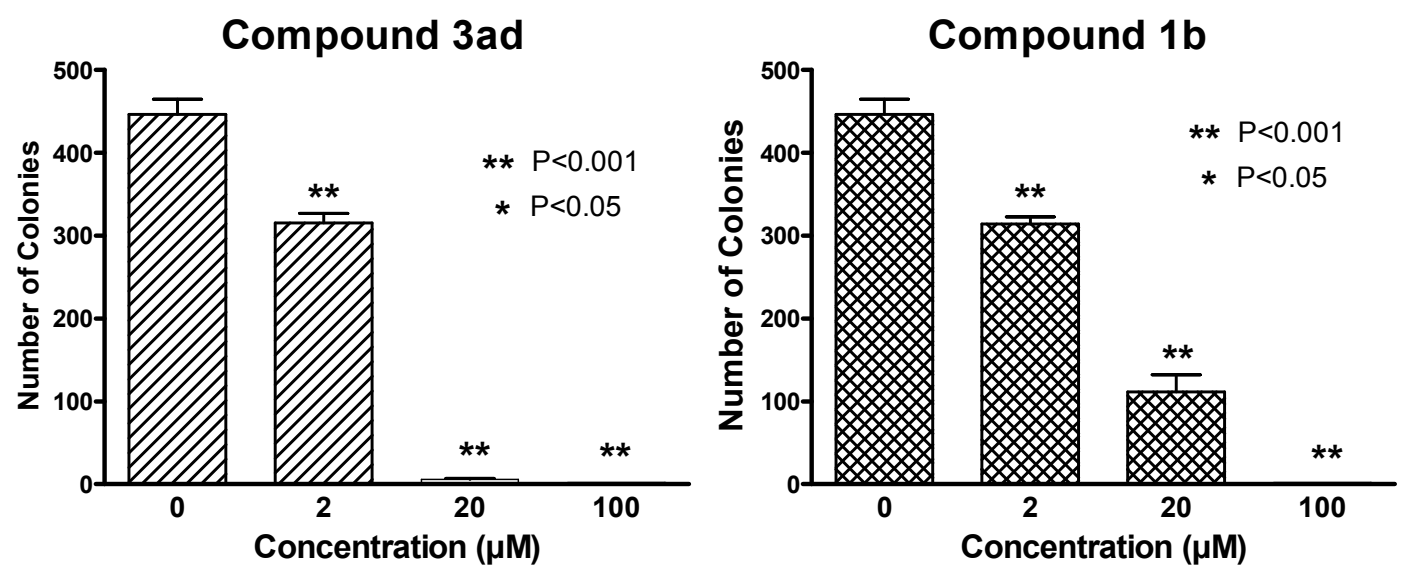

Figure 2-8. Quantified results of colony formation assay with compounds $3 \mathrm{ad}$ and $1 \mathrm{~b}$ treatment. 
eight mice. The results are shown in Figure 2-9. Compound $1 \mathrm{~b}$ at $5 \mathrm{mg} / \mathrm{kg}$ showed moderate melanoma tumor growth inhibition. After 22 days of treatment, the percentage of tumor reduction was $25 \%$. At a higher dose of $10 \mathrm{mg} / \mathrm{kg}$, compound $1 \mathrm{~b}$ showed significant melanoma tumor growth inhibition with $62 \%$ of tumor reduction. When comparing with DTIC at a dose of $60 \mathrm{mg} / \mathrm{kg}$, which inhibited tumor growth by $42 \%$ at the end of treatment, compound $1 \mathrm{~b}$ clearly showed superior activity in this human melanoma xenograft model. All mice displayed normal activities, and no significant body weight loss was observed during the experiment.

\subsection{Conclusions}

ATCAA analogs represent a novel class of potent and selective cytotoxic agents for melanoma. Initial analysis indicated that the antineoplastic activity is attributed to their ability to induce DNA break and LDH release in melanoma cells. These SAR studies revealed that the potency of the compounds depends strongly on the chain length and substitutions on the phenyl ring. Our current lead compound is highly potent and moderately selective when compared with Sorafenib, a drug used extensively in clinical trials for melanoma.

Dr. Yan Lu then synthesized a series of ATCAA compounds and thiazolidine ring-opened analogues. Chemical modification and structure-activity relationship of ATCAA compounds were investigated with different substituted 2-phenyl, thiazolidine ring, 4-position linkage, and 4-carboxamide groups based on biological evaluation against melanoma cells. Some compounds are very promising agents against melanoma. Compounds $1 \mathrm{~b}$ and $3 \mathrm{ad}$ effectively inhibited A375 melanoma colony formation. Compound $1 \mathrm{~b}$ at a dose of $10 \mathrm{mg} / \mathrm{kg}$ significantly inhibited melanoma tumor growth in vivo and showed higher efficacy than did DTIC at a dose of $60 \mathrm{mg} / \mathrm{kg}$. These compounds did not seem to work via the LPA-GPCR pathway.

Mr. Jianjun Chen also synthesized new imidazole and imidazoline analogs, based on our previous studies, by focusing on the structure activity relationship studies of the central five-member ring. Although the current compounds displayed lower potency when compared with our lead thiazolidine analogs, they may have the distinct advantage of being more stable in vivo with the reduced necessity of chiral separations. Some of these new compounds have activity similar to Sorafenib. 

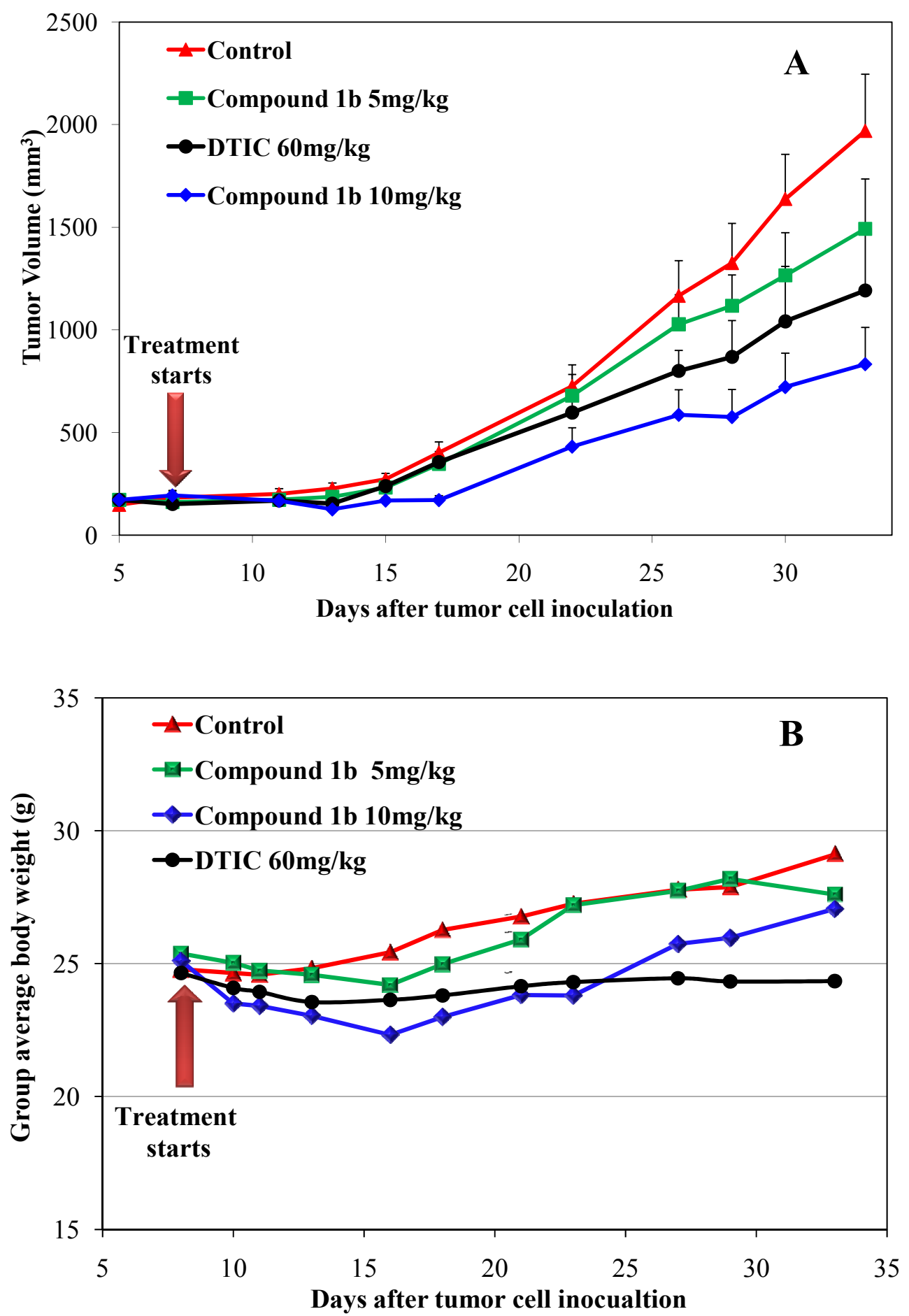

Figure 2-9. In vivo anti-melanoma test of compound $1 \mathrm{~b}$ on athymic nude mice $(\mathrm{n}=8$ per group) bearing A375 xenograft.

A: Tumor growth curve of control, compound $1 \mathrm{~b}$ and DTIC treatment. DTIC was used as positive control. B: Average body weight change in control and treatment groups. 


\section{CHAPTER 3. IDENTIFYING NOVEL MOLECULAR STRUCTURES FOR ADVANCED MELANOMA BY LIGAND-BASED VIRTUAL SCREENING*}

\subsection{Introduction}

In our ongoing efforts to search for small molecules as potential therapeutic agents for advanced melanoma, our team recently discovered a new series of thiazole analogs that showed very potent activity against melanoma cells in vitro (110). One of the best compounds in this series has an $\mathrm{IC}_{50}$ value below $60 \mathrm{nM}$. Screening results from the National Cancer Institute (NCI-60 screening) for our lead compound, LY-1-100, indicated nanomolar antiproliferative activity for all the cancer cell lines tested. Preliminary mechanism of action studies on this series of compounds indicated that they may interact with microtubules (110). In vivo testing with melanoma tumors showed substantial growth inhibitory activity for this series of compounds. To further expand our understanding of structure-activity relationships and to potentially identify new platforms for active compounds, Dr. Wei Li and Dr. William Seibel explored a compound library from the University of Cincinnati's (UC) Drug Discovery Center, which contains 342,910 small molecules. All compounds are available to us for testing via an established agreement. Usually, compounds are shipped within 2 days of request. Therefore, I can obtain any compound I select easily for biological testing. Although other compound libraries may have more entries than does the UC library, often availability of the compounds is an issue. Therefore, I chose this library for our current studies.

In this chapter, I will discuss two ligand-based virtual screening approaches using the structure of our lead molecule (Figure 3-1): 1) similarity search based on atom connectivity using Scitegic Pipeline Pilot software (Accelrys Software, Inc., San Diego, CA) and 2) similarity search based on molecular shape using Schrodinger software (Schrodinger, Inc., New York, NY). Results showed that these two approaches are highly complementary and lead to different active molecular structures. These structures are quite suitable for further structural modification and provide new platforms for our anticancer drug discovery efforts.

\subsection{Experimental Methods}

\subsubsection{Validation of Connectivity Similarity Search}

To validate the connectivity similarity search approach, Dr. Wei Li first established a relatively small testing compound library. This library contains 22 known compounds similar to LY-1-100, 10 known dissimilar compounds, and a diverse set of 2000 compounds pulled from the University of Cincinnati Drug Discovery Center

* This chapter adapted with permission from Wang Z, Lu Y, Seibel W, et al. Identifying novel molecular structures for advanced melanoma by ligand-based virtual screening. J Chem Inf Model 2009;49:1420-7. 


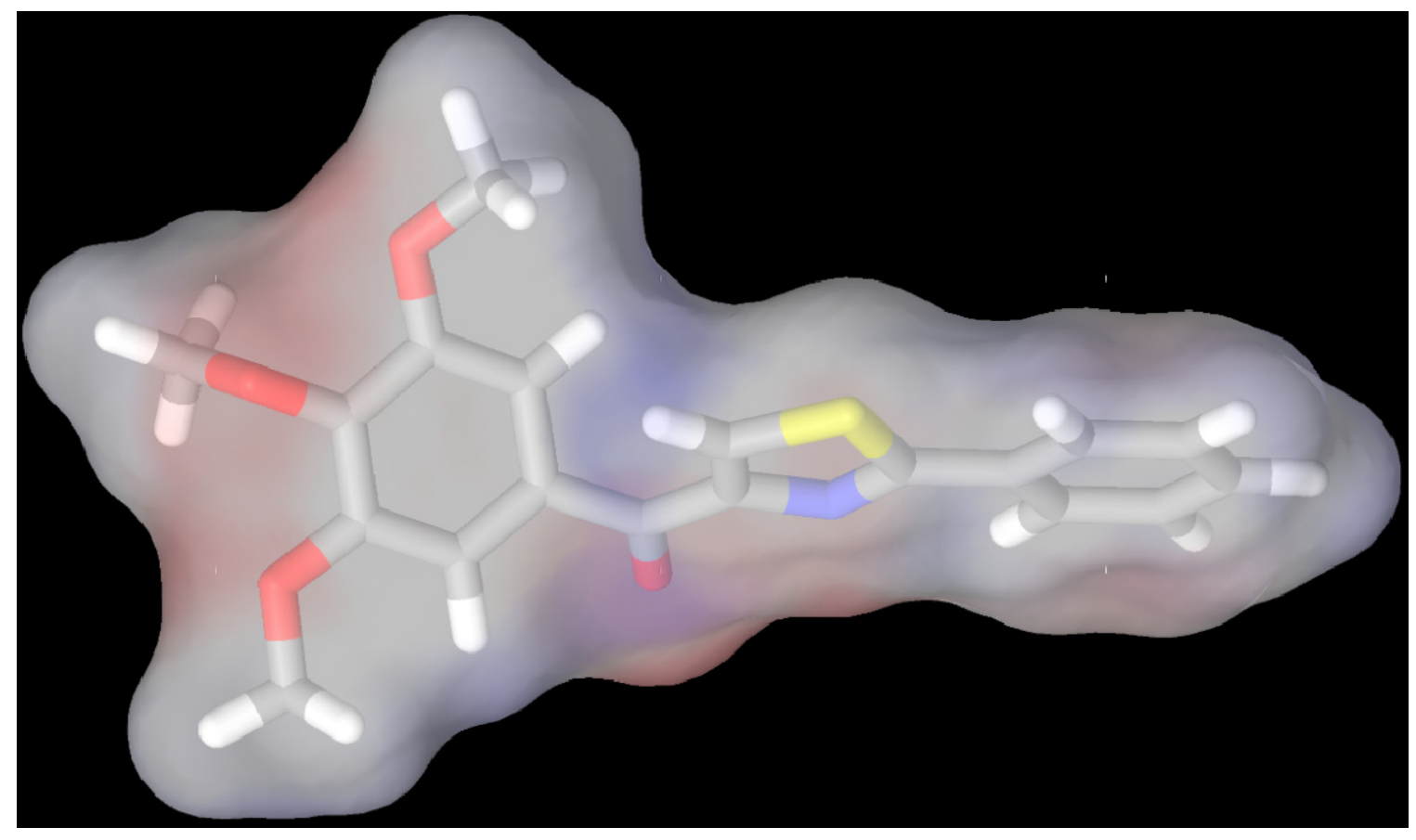

Figure 3-1. Structure and molecular surface of lead compound, LY-1-100. This figure was prepared by Dr. Wei Li, Department of Pharmaceutical Sciences, University of Tennessee Health Science Center. 
Compound Library (totally 342,910 compounds). These 2000 compounds were selected to be "druglike" in that they adhered to Lipinski rules and were filtered from a wide variety of functional groups. Dr. Wei Li seeded the known 32 compounds in the small testing library so they could be evenly distributed through the database file. Then Dr. Seibel conducted a connectivity similarity search for lead compound LY-1-100 against the 2032 testing compound library. The small compound library was subjected to five similarity filters in parallel using the ECFP2, ECFP4, ECFC6, FCFP4, and FCFP6 property sets and Tanimoto distances using LY-1-100 as the lead structure (111-113). The top 400 compounds most similar to LY-1-100 from each of these operations were ranked by calculated similarity; then an average rank for each compound across the methods was calculated. The detailed algorithm of the search is shown in Figure 3-2.

\subsubsection{Atom Connectivity-Based Virtual Screening}

A protocol was designed within Accelrys's Pipeline Pilot in the UC Drug Discovery Center. The entire compound library was subjected to the same five similarity filters in parallel as described in the validation process. Then an average rank for each compound was also calculated. Our experience suggests that each of the property sets has some inherent strengths and weaknesses with respect to any given structure, so we used the above multiple, parallel similarity analysis to ensure that the optimal compounds by any method were not missed. The detailed algorithm of the search is shown in Figure 3-2.

\subsubsection{Validation of Shape Similarity Search}

Dr. Wei Li also tested our shape-based virtual screening using the same test database. The lead compound, LY-1-100, has a relatively rigid structure. So it has less conformer than other molecules. Therefore, Dr Li chose its lowest energy conformer at an environment of $\mathrm{pH} 7.4$ as an active conformation, which is consistent with our recently reported crystal structure of LY-1-100 (110).

All structures in this database were first prepared with the Ligprep software model (Schrodinger, Inc., New York, NY). During this preparation step, hydrogen atoms were explicitly added, all possible ionization states were generated between $\mathrm{pH} 5.0$ and $\mathrm{pH} 9.0$ using the Ionizer module, and the 3D molecular structures were minimized with OPLS_2005 forcefield. Tautomers were also generated in this step. All structures were then subjected to shape similarity search using the Phase software module. Each structure was allowed to sample up to 100 conformers for which molecular shapes were calculated and compared with that of LY-100. Similarity scores were ranked from most similar to least similar. 


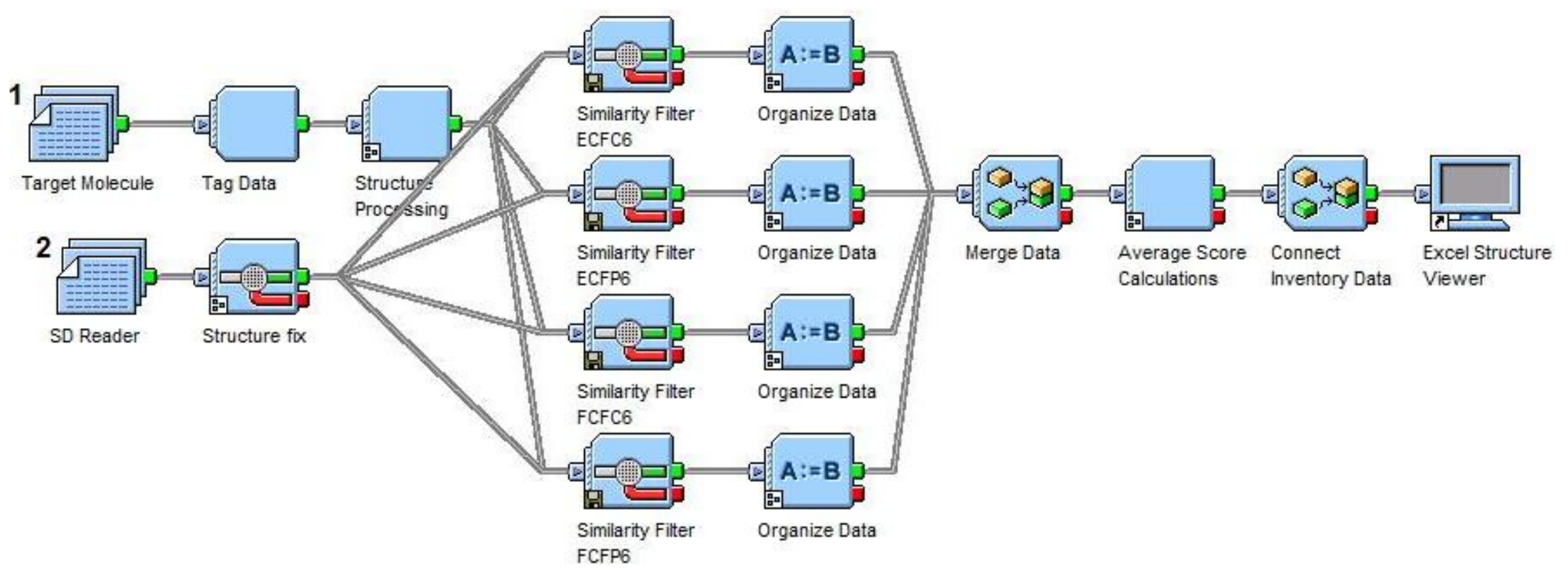

Figure 3-2. General protocol flowchart for virtual screening validation and virtual screening.

This figure was prepared by Dr. William Seibel, University of Cincinnati Drug Discovery Center, University of Cincinnati. 


\subsubsection{Molecular Shape-Based Virtual Screening}

To make the intensive computation of shape-based virtual screening manageable for these 342,910 compounds, the database was first broken down into 17 smaller databases. Each small database contained about 20,000 molecules and was subsequently prefiltered to remove molecules containing reactive groups. Molecules in each small database were then prepared by the Ligprep software module to generate proper 3D structures with all possible ionization states between $\mathrm{pH} 5.0$ and $\mathrm{pH} 9.0$. Typically 30,000 to 50,000 structures were produced from this step for each database. Subsequently, 100 conformations were generated for each of the structures in the database, and the shape of each conformation was then compared to that of LY-1-100. A normalized shape similarity value was computed for each molecular conformation relative to that of LY-1100, with 0 the most dissimilar and 1 the exact same shape. Dr. Li set the threshold for a hit as a shape similarity greater than 0.7 regardless of surface property to keep the maximum hits. All hits were ranked down from the most similar hit. These calculations were repeated for each small database for all the molecules in the original database.

\subsubsection{Biological Assay and Data Processing}

Hits from the two similarity searches were analyzed and their structures examined. The top hits were requested from the UC Drug Discovery Center for a subsequent two-step in vitro assay. The first step was a fixed drug concentration screening assay on two melanoma cell lines. Compounds that killed at least $50 \%$ of both cancer cells at $10 \mu \mathrm{M}$ were selected for the second step, and their $\mathrm{IC}_{50}$ values on two melanoma cell lines and one control cell line were determined. Human malignant melanoma cells A375 and mouse melanoma cells B16-F1 were used to evaluate the compounds' in vitro anticancer activity. Both A375 cells and B16-F1 cells were obtained from American Type Culture Collection (Manassas, VA). All cell lines were cultured in DMEM media (Cellgro Mediatech, Inc., Herndon, VA), supplemented with 10\% FBS (Cellgro Mediatech, Inc.), 1\% antibiotic/antimycotic mixture (Sigma-Aldrich, Inc., St. Louis, MO), and bovine insulin ( $5 \mu \mathrm{g} / \mathrm{mL}$; Sigma-Aldrich). Cultures were maintained at $37^{\circ} \mathrm{C}$ in a humidified atmosphere containing $5 \% \mathrm{CO}_{2}$.

For the initial screening step, A375 and B16-F1 cells were seeded to 96-well plates at a density of 4000 5000 cells/well, respectively. After cells adhered to the plate, media were changed and test compounds were added together with media at a fixed concentration of $10 \mu \mathrm{M}$ in duplicate. After 48-h incubation, cell viability was evaluated by using the CellTiter 96® AQueous One Solution Cell Proliferation Assay (114). This assay is based on the bioreduction of MTS [3-(4, 5-dimethylthiazol-2-yl)-5-(3carboxymethoxyphenyl)-2-(4-sulfophenyl)-2H-tetrazolium]. This tetrazolium dye can be converted by living cells into a colored formazan product that is soluble in tissue culture medium and, therefore, provides a sensitive readout of cell life or death that can be monitored spectroscopically. After incubating cells with compounds for $48 \mathrm{~h}, 20 \mu \mathrm{L}$ of CellTiter 96 AQueous One Solution Reagent was pipetted into each well, which contained the samples in $100 \mu \mathrm{L}$ of culture medium. Plates were incubated for another 
$1.5 \mathrm{~h}$ at $37^{\circ} \mathrm{C}$ in a humidified, $5 \% \mathrm{CO}_{2}$ atmosphere. Absorbance was recorded at $490 \mathrm{~nm}$ in a BioTek EL800 96-well plate reader (BioTek Instruments, Inc., Winooski, VT). Each compound's cell killing rate was normalized against a no-treatment control.

The second step was to further measure the $\mathrm{IC}_{50}$ s of active compounds selected from the initial step. I used the activity on fibroblast cells as a control to determine the selectivity of these compounds between cancer cells and normal cells. Human dermal fibroblast cells were purchased from Cascade Biologics, Inc., (Portland, OR) and cultured in the same condition as the two melanoma cell lines. After cells were seeded on 96-well plates, they were exposed to a wide range of eight concentrations of each compound in quadruplicate to determine the $\mathrm{IC}_{50}$ values. Then the CellTiter 96 AQueous One Solution Cell Proliferation Assay was used to measure cell viability. $\mathrm{IC}_{50}$ values were calculated by nonlinear regression analysis using GraphPad Prism (GraphPad Software, San Diego, CA) $(115,116)$. Each assay was repeated three times on different occasions.

\subsection{Results and Discussion}

\subsubsection{Validation of Connectivity Similarity Search}

Results from the connectivity search using the test database showed that the first 19 most similar compounds were from the 22 seeded similar compounds according to the consensus score (average rank). The lowest rank of all seeded similar compounds was

$31^{\text {st }}$ in the search results. The highest ranking "dissimilar" compound was ranked at $131^{\text {st }}$. This is approximately $6.5 \%$ of the way through the small 2032 compound database, making it analogous to the $22,106^{\text {th }}$ ranked compound from the original search of the 342,910 compounds library. This validation exercise clearly showed that similar compounds are easily ranked higher than dissimilar compounds by the connectivity indices-based methods we used.

\subsubsection{Connectivity Similarity Search}

The connectivity searches produced a combined total of 1292 compounds. To illustrate that each property set identified somewhat different similar structures, only 13 compounds were in common among all five searches, 49 in common among four searches, and 160 in common among three searches. Results were consistent with a limited number of highly similar compounds and a fairly rapid digression to relatively low-similarity compounds. Out of these 1292 compounds, I selected the top-ranked 110 compounds, middle-ranked 110 compounds, and bottom-ranked 110 compounds to screen their activity on the two cancer cell lines: A375 and B16-F1. Our decision to select compounds from the top-, middle-, and bottom-ranked compounds was not only to ensure testing of any highly similar (and therefore likely to be active) compounds (top-ranked) but also to search for active compounds of more divergent structure (middle- and lowranked compounds). Each of the 330 compounds was incubated with cells at a 
concentration of $10 \mu \mathrm{M}$ for $48 \mathrm{~h}$ in duplicate. Percentages of cell death induced by these compounds are shown in Figure 3-3. Three active compounds were identified from the 110 top-ranked compounds (UC-297549, UC-791794, and UC-791475), one active compound from the 110 middle-ranked compounds (UC-406551), and four active compounds from the 110 bottom-ranked compounds (UC-791814, UC-791792, UC792257, and UC-193691). IC 50 values for these active compounds were subsequently measured on A375, B16-F1, and fibroblast cells (Table 3-1). The best compound identified, UC-297549, had an $\mathrm{IC}_{50}$ value less than $1 \mu \mathrm{M}$ on A375 cells and good selectivity between cancer cells and normal cells. Very interestingly, almost all of these compounds contained a basic terminal amine group close to an aromatic ring. This feature is different from the lead compound structure (LY-1-100), suggesting that adding a properly positioned amine group may enhance the activity of LY-1-100. Another important feature revealed from Table 3-1 is that a basic terminal amine group may help increase selectivity. Among the identified eight active compounds, only one did not have the basic terminal amine group (UC-406551). The selectivity of this compound was worse than that of the lead compound. All other seven active compounds, which have the basic terminal amine group, had better selectivity than our lead compound. Finally, a basic terminal amine group is also expected to have the added benefit of a reduced $\log \mathrm{P}$ value and improved water solubility.

With this in mind, Dr. Yan Lu synthesized a new analog of LY-1-100 in which an amino group was added to the para-position of the phenyl ring (compound LY-2-183H). To our great satisfaction, LY-2-183H was more active and selective than LY-1-100 was (Table 3-1). As expected, LY-2-183H had much better water solubility, which is an important factor for future in vivo animal testing. Further optimization of this structure is currently in progress.

\subsubsection{Additional Analog Screening Based on Active Compounds Identified from Connectivity-Based Screening}

Encouraged by the structure of the initial connectivity screening and using the same protocol as described above in combination with substructural analysis, I selected an additional 40 compounds whose structures were close to the 8 active compounds. When I tested the activity for these 40 compounds on A375 and B16-F1 cells, 4 active compounds (10\% hit rate, Figure 3-4) were identified (UC-792247, UC-792341, UC98514, and UC-831104). Their $\mathrm{IC}_{50}$ values against all three cell lines are shown in Table 3-2. While these four compounds were not as active as was UC-297549, they contained more structural features than LY-1-100 and may provide more opportunities for further structural modifications.

\subsubsection{Validation of Shape Similarity Search}

During the testing compound library preparation step, the Ligprep process generated 6767 distinct structures from the original 2032 entries. Thirty-five ionization 
Figure 3-3. In vitro screening results for compounds identified from atom connectivitybased virtual screening.

Percentage of cell death resulted from compound treatment was normalized by nontreatment control. Each bar represents the average of two replications. 


\section{Cytotoxicity of Compounds in Plate 624 on A375}

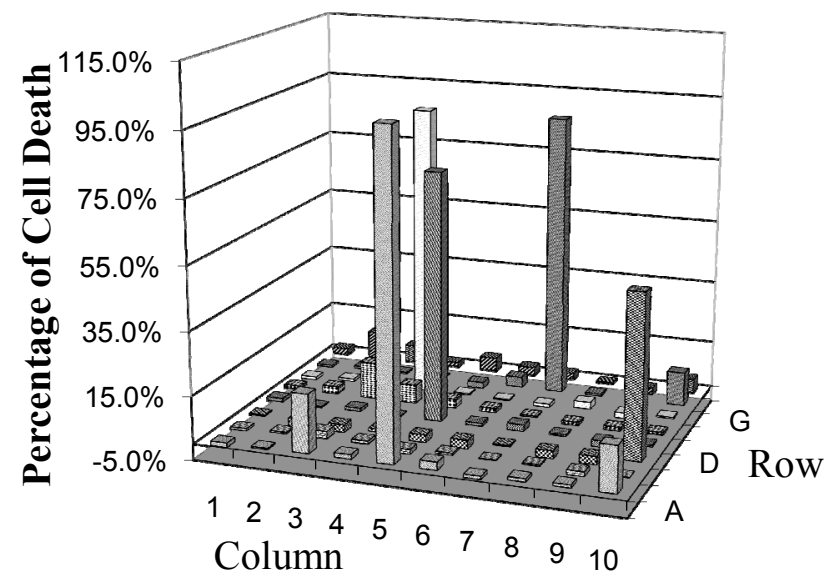

Cytotoxicity of Compounds in Plate 820 on A375

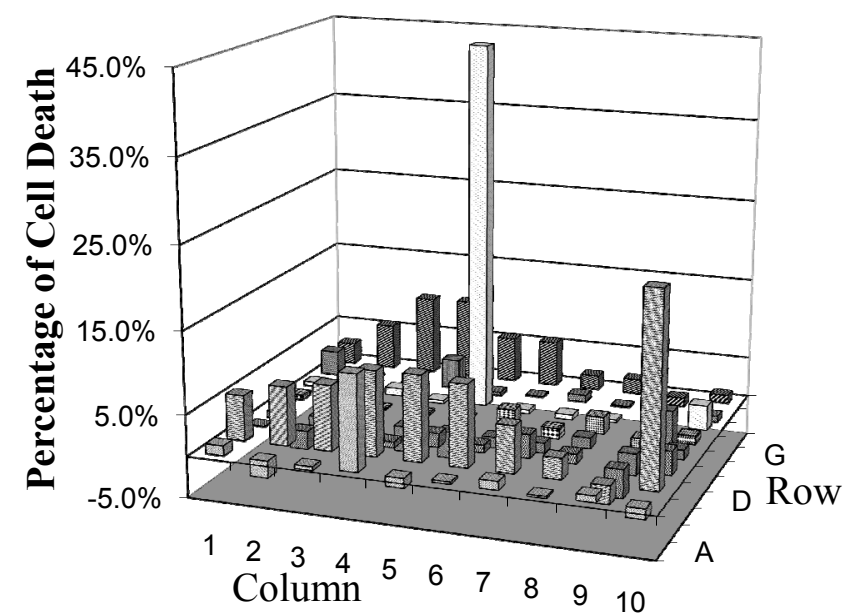

\section{Cytotoxicity of Compounds in Plate 624 on B16-F1}

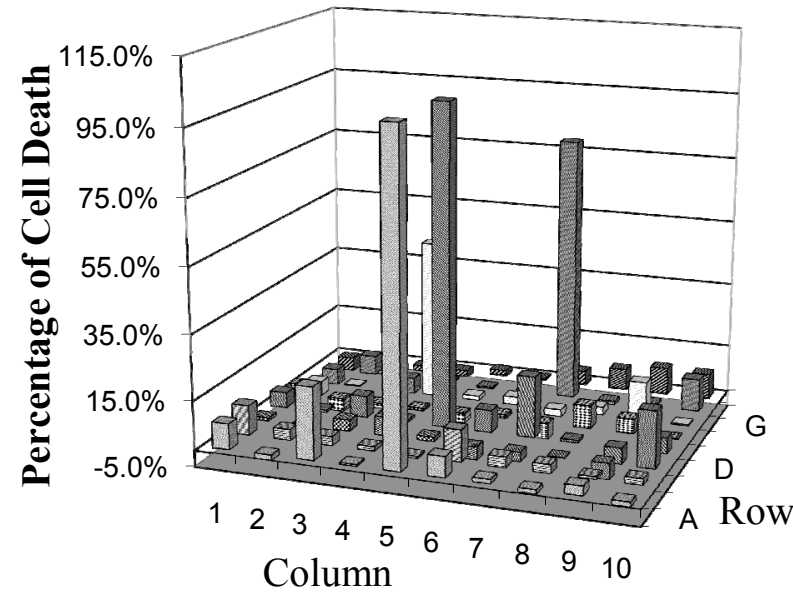

Cytotoxicity of Compounds in Plate 820 on B16-F1

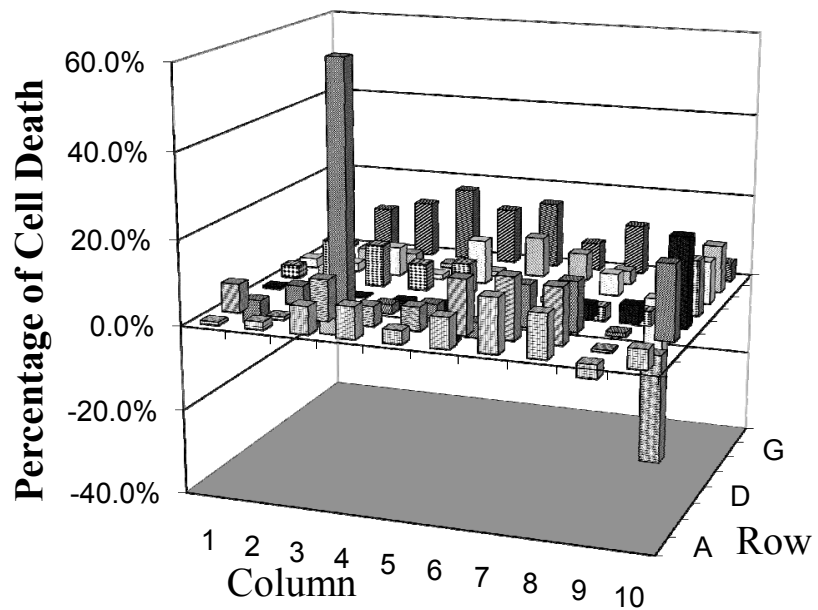




\section{Cytotoxicity of Compounds in Plate 821 on A375}

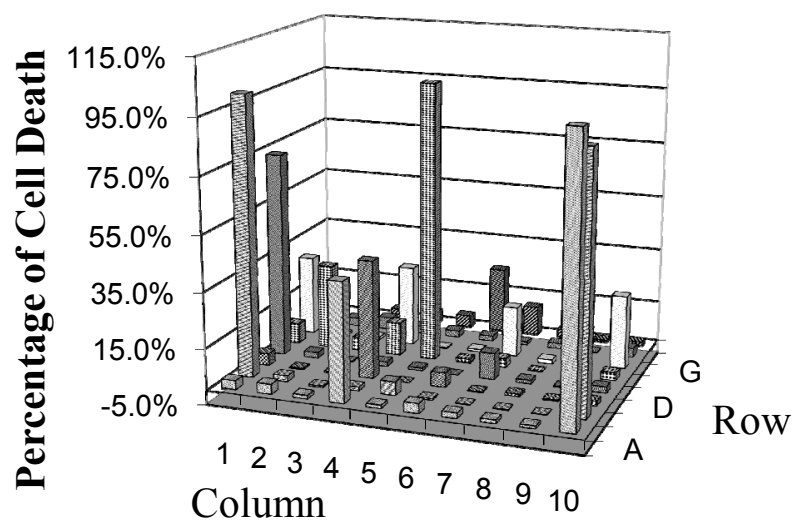

Cytotoxicity of Compounds in Plate 822 on A375

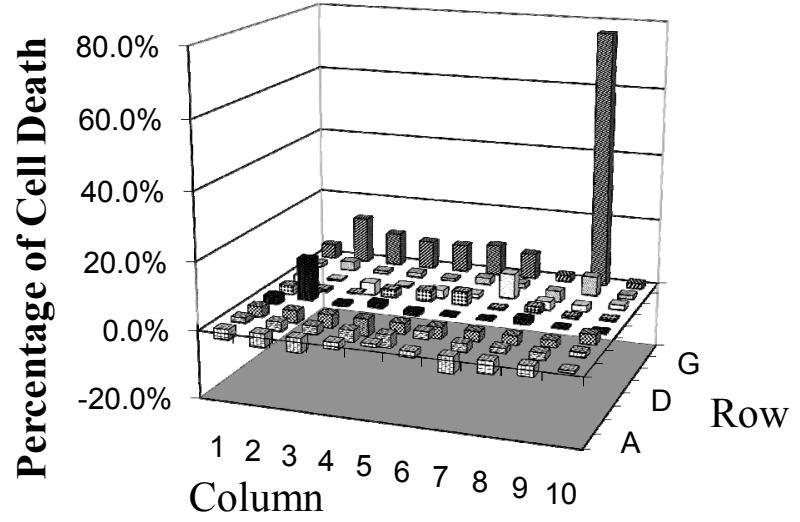

Cytotoxicity of Compounds in Plate 821 on B16-F1

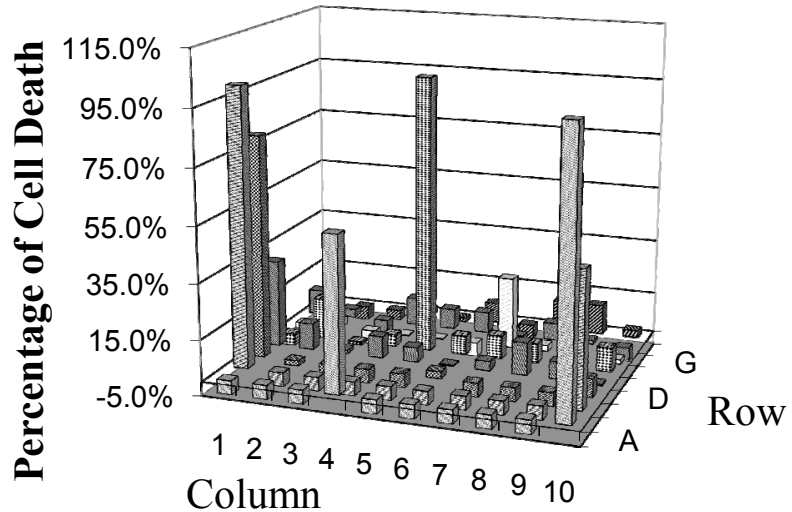

Cytotoxicity of Compounds in Plate 822 on B16-F1

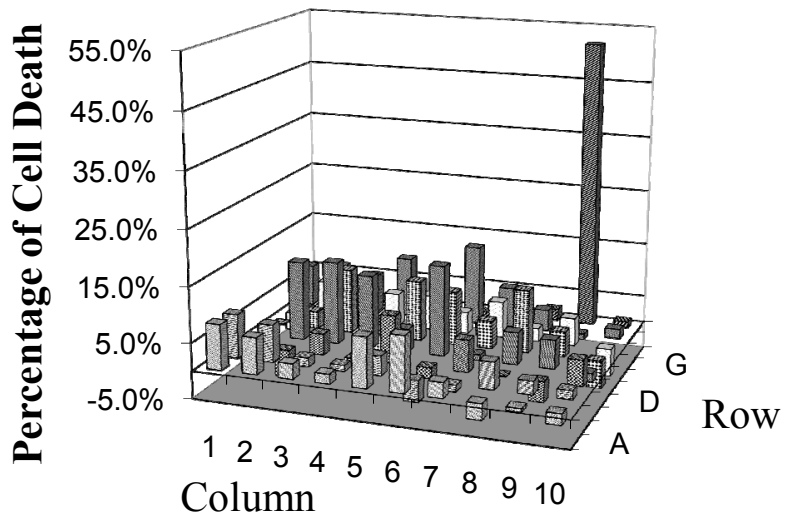

Figure 3-3. (continued) 
Table 3-1. $\mathrm{IC}_{50}$ of lead compound, eight active compounds identified from atom connectivity-based virtual screening, and newly synthesized compounds.

\begin{tabular}{|c|c|c|c|c|c|}
\hline \multirow{2}{*}{ UC number } & \multirow{2}{*}{ Structure } & \multicolumn{3}{|c|}{$\mathrm{IC}_{50} \pm \operatorname{SEM}(\mu \mathrm{M})(\mathrm{n}=3)$} & \multirow{2}{*}{ Selectivity* } \\
\hline & & B16-F1 & A375 & $\mathrm{HDFa}$ & \\
\hline $\begin{array}{l}\text { LY-1-100 } \\
\text { (Lead } \\
\text { structure) }\end{array}$ & & $\begin{array}{c}55 \pm 5 \\
\mathrm{nM}\end{array}$ & $\begin{array}{c}28 \pm 5 \\
\mathrm{nM}\end{array}$ & $\begin{array}{c}35 \pm 4 \\
\mathrm{nM}\end{array}$ & 1 \\
\hline 297549 & & $12 \pm 2$ & $\begin{array}{c}500 \pm 100 \\
\mathrm{nM}\end{array}$ & $>20$ & $>3$ \\
\hline 791814 & & $8 \pm 1$ & $4 \pm 1$ & $8 \pm 1$ & 1 \\
\hline 791792 & & $9 \pm 1$ & $6 \pm 1$ & $10 \pm 1$ & 1 \\
\hline 791794 & & $9 \pm 1$ & $8 \pm 1$ & $9 \pm 1$ & 1 \\
\hline 792257 & & $10 \pm 1$ & $10 \pm 1$ & $>20$ & $>2$ \\
\hline 791475 & & $11 \pm 1$ & $9 \pm 1$ & $18 \pm 2$ & 2 \\
\hline 406551 & & $11 \pm 1$ & $14 \pm 1$ & $4 \pm 0$ & 0.3 \\
\hline 193691 & & $7 \pm 1$ & $8 \pm 0$ & $21 \pm 3$ & 3 \\
\hline LY-2-183H & & $\begin{array}{c}14 \pm 4 \\
\mathrm{nM}\end{array}$ & $\begin{array}{c}11 \pm 3 \\
\mathrm{nM}\end{array}$ & $\begin{array}{c}18 \pm 2 \\
n M\end{array}$ & 2 \\
\hline
\end{tabular}

*Selectivity is defined as the ratio of $\mathrm{IC}_{50}$ on fibroblast over the $\mathrm{IC}_{50}$ average in the two cancer cell lines. 


\section{Cytotoxicity of Compounds in Plate 848 on A375}

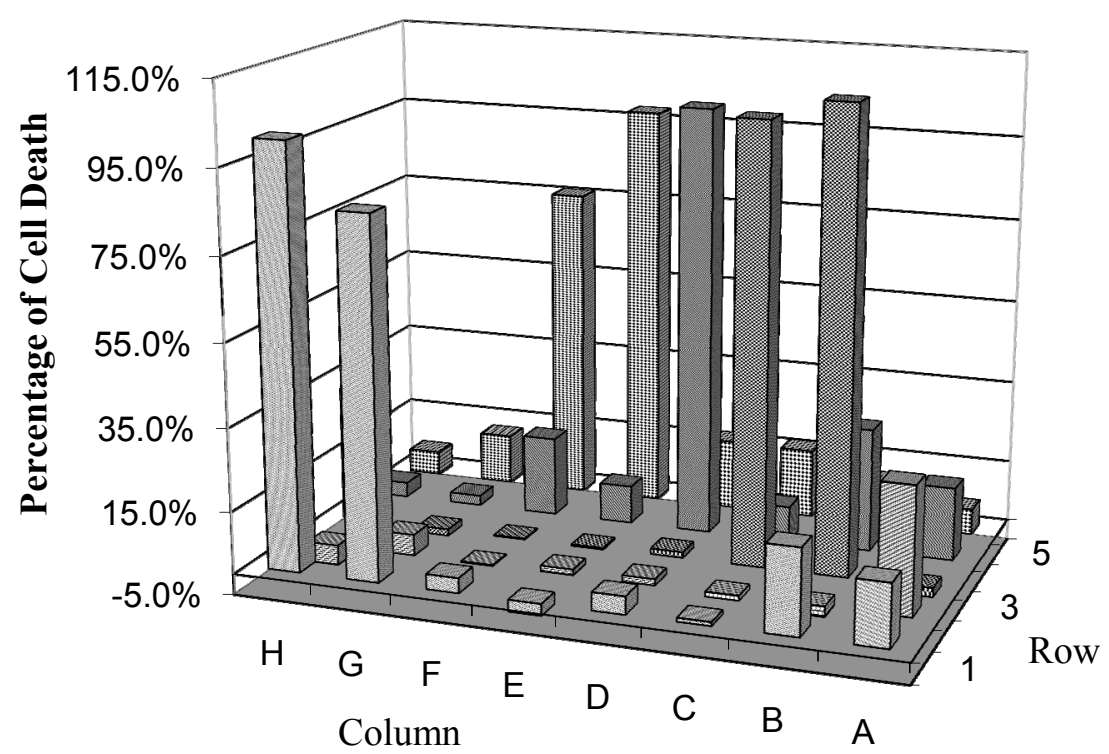

\section{Cytotoxicity of Compounds in Plate 848 on B16-F1}

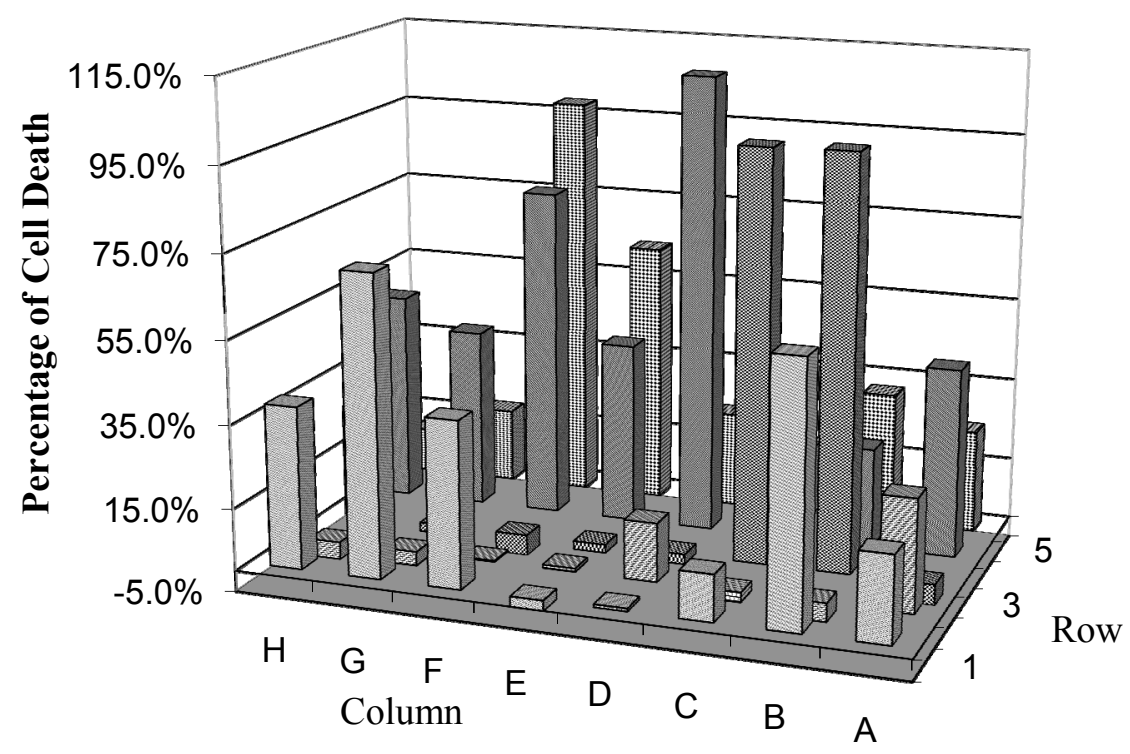

Figure 3-4. In vitro screening results for compounds identified from further atom connectivity similarity search based on previously identified active compounds. All compounds were tested at $10 \mu \mathrm{M}$ concentration on A375 and B16-F1 melanoma cells. Each bar represents the average of two replications. 
Table 3-2. $\mathrm{IC}_{50}$ of active compounds identified from further atom connectivity similarity search based on previously identified active compounds.

\begin{tabular}{llllll} 
UC & & \multicolumn{2}{c}{$\mathrm{IC}_{50} \pm \mathrm{SEM}(\mu \mathrm{M})(\mathrm{n}=3)$} & \\
\cline { 3 - 6 } number Structure & $\mathrm{B} 16-\mathrm{F} 1$ & $\mathrm{~A} 375$ & $\mathrm{HDFa}$ & \\
\hline
\end{tabular}

*Selectivity is defined as the ratio of $\mathrm{IC}_{50}$ on fibroblast over the $\mathrm{IC}_{50}$ average in the two cancer cell lines. 
states were generated for the 32 seeded molecules, including 24 similar structures (protonated and unprotonated forms for both LY-2-103 and LY-2-84), and 11 dissimilar structures (Figure 3-5, seeded similar structures are highlighted in yellow, and dissimilar structures are highlighted in red). Validation search results for the seeded structures are listed in Figure 3-5. From these results, I can see that the top 26 entries from this search contained 20 of the 24 seeded similar structures $(86 \%$ similar structures from $0.35 \%$ of total database). The four remaining similar structures ranked at 107, 255, 281, and 1508 . Twenty-three out of $24(96 \%)$ similar structures were found within the $4.2 \%$ of the total structures in this sample database. The saturated thiazole ring and the amide bond in LY2-74 are responsible for its low similarity ranking of 1508, when compared with the molecular shape of LY-100 (Figure 3-1). This structure also showed the worst similarity rank in the connectivity-based similarity search (rank $31^{\text {st }}$ ). In contrast, the ranks for the 10 dissimilar structures spanned from 332 to 6712 out of the 6767 structures. In summary, I found that this shape similarity search approach could identify similar compounds, which provides reasonable validation for screening the entire database.

\subsubsection{Shape Similarity Search}

The classical concept of lock and key interaction between a receptor and its ligand indicates that molecular shape is a critical factor for high binding affinity. Similarities in connectivity and molecular shape have overlap but also have their own individual spaces. To explore potentially new platforms based on the molecular shape of LY-1-100, Dr. Li performed shape similarity searches against this database. These searches produced a little over 5000 structures that had a similarity score larger than 0.7 and did not contain any reactive groups. I selected the 88 top-ranked compounds and performed an initial single concentration screening (Figure 3-6). Two active compounds (UC-521092 and UC-398535), which are very close analogs, were identified, and their $\mathrm{IC}_{50} \mathrm{~s}$ are shown in

Table 3-3. The tight criterion (similarity score must be at least 0.7 ) was likely responsible for limiting greater variations for identified structures.

Although still not as active as LY-1-100, these two compounds were more potent than compounds identified from connectivity-based virtual screening, with $\mathrm{IC}_{50}$ values in the nanomolar range. One important hint revealed from this search was that a properly constructed amide linkage between the five-member ring and the trimethoxyphenyl ring may be very beneficial to anticancer activity. Previously, Dr. Lu made one compound (LY-2-30) containing an amide bond that was not active. Based on results of this screening, Dr. Lu prepared another analog in which the direction of the amide bond was reversed to directly connect the carbonyl group to the trimethoxyphenyl ring (LY-2173b-OTs). The activity of this compound was greatly improved (Table 3-3).

The high activity of compounds identified from this shape similarity search likely resulted from the very close structural features to LY-1-100. Obviously, this similarity also limited their structural variations; therefore, a dramatically different scaffold from LY-1-100 will be difficult to obtain. Hence, at least for this database, shape similarity search will not provide completely new molecular structures. On the other hand, 
Figure 3-5. Seeded structures in validation testing library with shape similarity ranks. Seeded similar structures are highlighted in yellow. Dissimilar structures are highlighted in red. 
<smiles>COc1cc(C(=O)c2csc(-c3ncccn3)n2)cc(OC)c1OC</smiles>

TN Name: LY-2-122

title: 855555

Shape Sim: 0.956346823882

entry id: 8910

Similarity Rank: 1<smiles>COc1cc(/C(=N/N)c2csc(-c3ccccc3)n2)cc(OC)c1OC</smiles>

TN Name: LY-2-176A

title: 525555

Shape Sim: 0.942492900121

entry id: 8913

Similarity Rank: 4<smiles>COc1cc(C(=O)c2coc(-c3ccccc3)n2)cc(OC)c1OC</smiles>

TN Name: LY-2-163

title: 885555

Shape Sim: 0.93486642428

entry id: 8916

Similarity Rank: 7<smiles>COc1cc(C(=O)c2csc(-c3cc[nH+]cc3)n2)cc(OC)c1OC</smiles>

TN Name: LY-2-84

title: 825555

Shape Sim: 0.967433373886

entry id: 8909

Similarity Rank: 10<smiles>COc1cc(C(=O)c2csc(-c3ccc(N)cc3)n2)cc(OC)c1OC</smiles>

TN Name: LY-2-183

title: 735555

Shape Sim: 0.95250193819

entry id: 8911

Similarity Rank: 2<smiles>COc1cc(C(=O)c2csc(-c3ccsc3)n2)cc(OC)c1OC</smiles>

TN Name: LY-2-105

title: 795555

Shape Sim: 0.940105637529

entry id: 8914

Similarity Rank: 5<smiles>COc1cc(/C(=N/O)c2csc(-c3ccccc3)n2)cc(OC)c1OC</smiles>

TN Name: LY-2-173A

title: 495555

Shape Sim: 0.897033632287

entry id: 8917

Similarity Rank: 8<smiles>COc1ccc(-c2nc(C(=O)c3cc(OC)c(OC)c(OC)c3)cs2)cc1OC</smiles>

TN Name: LY-2-52

title: 765555

Shape Sim: 0.885174367947

entry id: 8919

Similarity Rank: 11<smiles>COc1cc(C(=O)c2csc(-c3ccc(F)cc3)n2)cc(OC)c1OC</smiles>

TN Name: LY-2-45

title: 615555

Shape Sim: 0.952469090673

entry id: 8912

Similarity Rank: 3<smiles>COc1cc(C(=O)c2csc(-c3ccc(C)cc3)n2)cc(OC)c1OC</smiles>

TN Name: LY-2-104

title: 645555

Shape Sim: 0.937374050216

entry id: 8915

Similarity Rank: 6<smiles>COc1cc(C(=O)N2CCC(c3ccccc3)CC2)cc(OC)c1OC</smiles>

TN Name: LY-2-194

title: 945555

Shape Sim: 0.891212697874

entry id: 8918

Similarity Rank: 9<smiles>COc1cc(C(=O)c2csc(-c3ccc(C(F)(F)F)cc3)n2)cc(OC)c1OC</smiles>

TN Name: LY-2-170

title: 675555

Shape Sim: 0.875733013519

entry id: 8920

Similarity Rank: 12 
<smiles>COc1cc(C(=O)[C@@H]2COC(c3ccccc3)=N2)cc(OC)c1OC</smiles>

TN Name: LY-2-165

title: 915555

Shape Sim: 0.87418013741

entry id: 8921

Similarity Rank: 13<smiles>COc1cc(NC(=O)c2csc(-c3ccccc3)n2)cc(OC)c1OC</smiles>

TN Name: LY-2-30

title: 555555

Shape Sim: 0.835902713766

entry id: 8925

Similarity Rank: 17

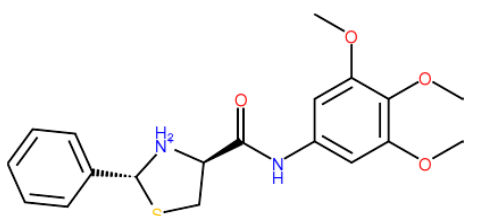

TN Name: LY-2-72

title: 435555

Shape Sim: 0.819756876003

entry id: 8929

Similarity Rank: 21<smiles>COc1cc(C(=O)c2cccc(-c3ccccc3)c2)cc(OC)c1OC</smiles>

TN Name: LY-2-127

title: 405555

Shape Sim: 0.748903854875

entry id: 9163

Similarity Rank: 255<smiles>COc1cc(C(=O)c2csc(-c3ccncc3)n2)cc(OC)c1OC</smiles>

TN Name: LY-2-84

title: 825555

Shape Sim: 0.874107443087

entry id: 8922

Similarity Rank: 14<smiles>O=C(c1ccccc1)c1csc(-c2ccccc2)n1</smiles>

TN Name: LY-2-97

title: 975555

Shape Sim: 0.823240844522

entry id: 8927

Similarity Rank: 19<smiles>O=C(c1ccccn1)c1csc(-c2ccccc2)n1</smiles>

TN Name: LY-2-103

title: 1005555

Shape Sim: 0.813796386954

entry id: 8934

Similarity Rank: 26<smiles>O=C(c1csc(-c2ccccc2)n1)c1cccc[nH+]1</smiles>

TN Name: LY-2-103

title: 1005555

Shape Sim: 0.746101642039

entry id: 9189

Similarity Rank: 281<smiles>COC(=O)c1ccc(-c2nc(C(=O)c3cc(OC)c(OC)c(OC)c3)cs2)cc1</smiles>

TN Name: LY-2-149

title: 705555

Shape Sim: 0.858375632951

entry id: 8923

Similarity Rank: 15<smiles>COc1cc(C(=O)Nc2csc(-c3ccccc3)n2)cc(OC)c1OC</smiles>

TN Name: LY-2-173b-OTs

title: 585555

Shape Sim: 0.820563760383

entry id: 8928

Similarity Rank: 20<smiles>COc1cc(NC(=O)[C@H]2CSC(c3ccccc3)=N2)cc(OC)c1OC</smiles>

TN Name: LY-2-28

title: 1035555

Shape Sim: 0.770542448596

entry id: 9015

Similarity Rank: 107<smiles>N#CC(=C(/N)Sc1ccccc1N)/C(C#N)=C(\N)Sc1ccccc1N</smiles>

TN Name: 10 other 9

title: 345555

Shape Sim: 0.74109407726

entry id: 9240

Similarity Rank: 332

Figure 3-5. (continued) 
<smiles>CC(C)C[C@H](NC(=O)CN)C(=O)N[C@@H](Cc1ccc(O)cc1)C(=O)[O-]</smiles>

TN Name: 10 other 4 title: 195555

Shape Sim: 0.700579174062

entry id: 9820

\section{Similarity Rank: 912}

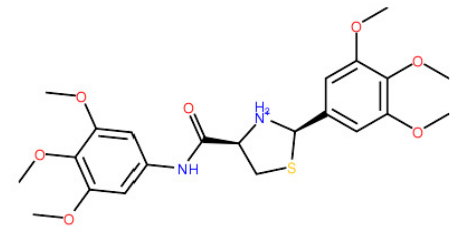

TN Name: LY-2-74

title: 465555

Shape Sim: 0.671585257095

entry id: 10416

Similarity Rank: 1508

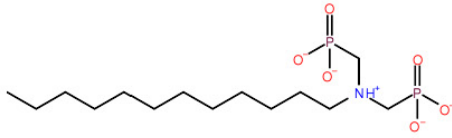

TN Name: 10 other 2

title: 135555

Shape Sim: 0.608588254613

entry id: 12307

\section{Similarity Rank: 3399}

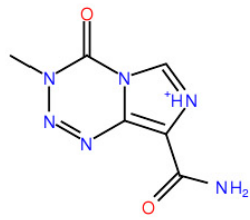

TN Name: 10 other 10

title: 375555

Shape Sim: 0.432871122727

entry id: 15613

Similarity Rank: 6705

Figure 3-5. (continued)<smiles>Cc1ccc(C(=O)C[C@H](NCc2ccccc2)C(=O)[O-])cc1</smiles>

TN Name: 10 other 5 title: 225555

Shape Sim: 0.698131477844 entry id: 9874

Similarity Rank: 966<smiles>CC(C)c1cccc(C(C)C)c1NC(=O)C[NH+](CC(=O)O)CC(=O)O</smiles>

TN Name: 10 other 1 title: 105555

Shape Sim: 0.669602313836 entry id: 10458

Similarity Rank: 1550<smiles>C=C(CCCCCCCC1CC1)CCC1CCCC(C)(C)C1</smiles>

TN Name: 10 other 6 title: 255555

Shape Sim: 0.603004968174 entry id: 12507

Similarity Rank: 3599<smiles>Cn1nnc2c(C(N)=O)ncn2c1=O</smiles>

TN Name: 10 other 10 title: 375555

Shape Sim: 0.4287030282 entry id: 15620

Similarity Rank: 6712<smiles>N[C@@H](Cc1ccc(Br)cc1)C(=O)NC(Cc1c[nH]c2ccccc12)C(=O)O</smiles>

TN Name: 10 other 3 title: 165555

Shape Sim: 0.684318135541 entry id: 10152

Similarity Rank: 1244<smiles>O=c1cc(N2CCOCC2)oc2c(-c3ccccc3)cccc12</smiles>

TN Name: 10 other 8 title: 315555

Shape Sim: 0.660778872054 entry id: 10673

Similarity Rank: 1765

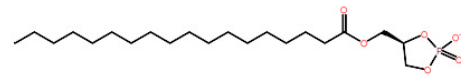

TN Name: 10 other 7

title: 285555

Shape Sim: 0.581513223034

entry id: 13258 


\section{Cytotoxicity of Compounds in Plate 561 on A375}

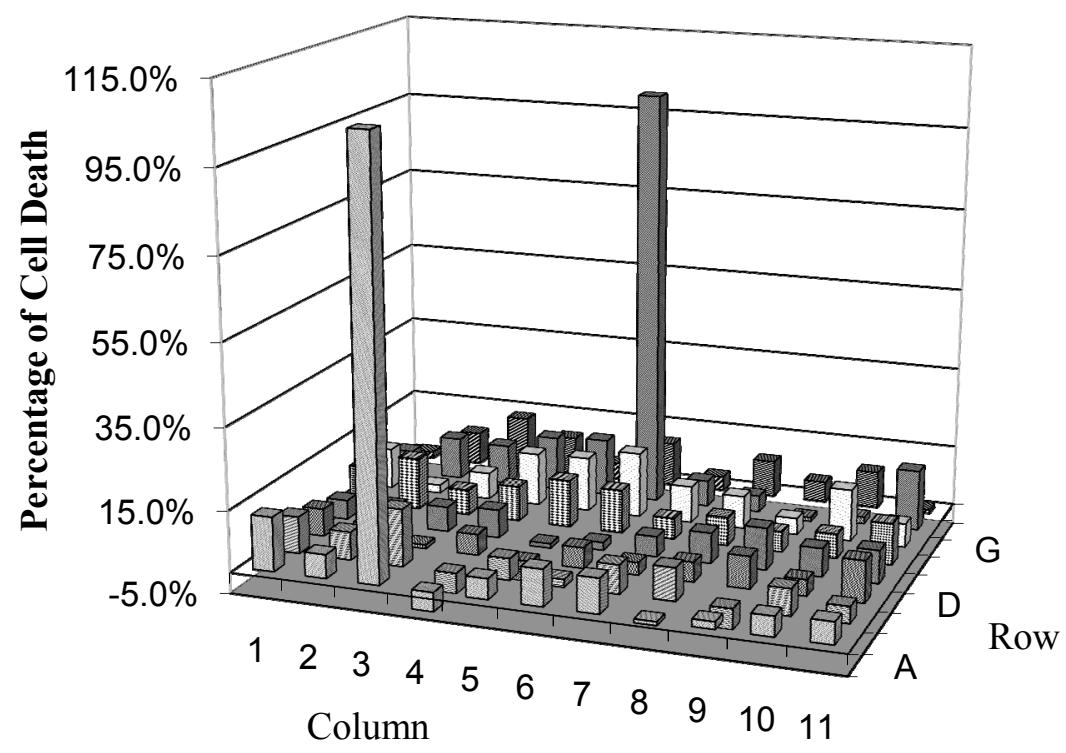

Cytotoxicity of Compounds in Plate 561 on B16-F1

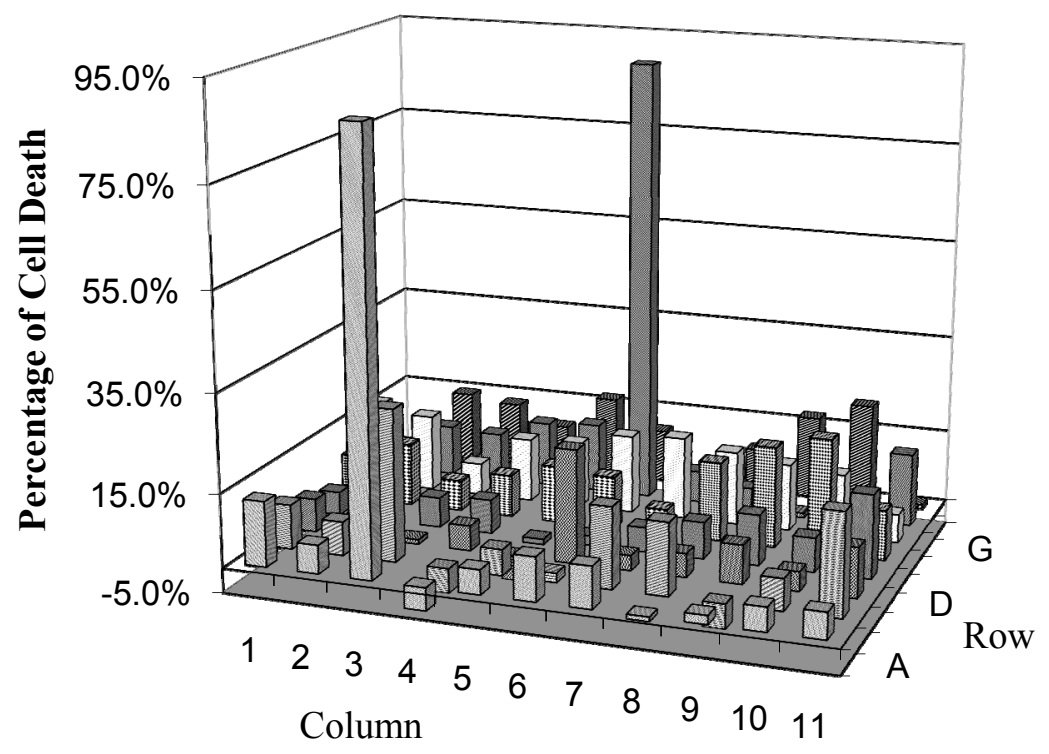

Figure 3-6. In vitro screening results for compounds identified from molecular shapebased virtual screening. 
Table 3-3. $\mathrm{IC}_{50}$ of active compounds identified from a shape similarity search, one inactive compound, and one newly synthesized compound based on the information get from shape similarity search.

\begin{tabular}{|c|c|c|c|c|c|}
\hline \multirow{2}{*}{ UC number } & \multirow{2}{*}{ Structure } & \multicolumn{3}{|c|}{$\mathrm{IC}_{50} \pm \operatorname{SEM}(\mu \mathrm{M})(\mathrm{n}=3)$} & \multirow{2}{*}{ Selectivity* } \\
\hline & & B16-F1 & A375 & HDFa & \\
\hline 521092 & & $\begin{array}{c}800 \pm 200 \\
\mathrm{nM}\end{array}$ & $\begin{array}{c}200 \pm 100 \\
\mathrm{nM}\end{array}$ & $\begin{array}{c}300 \pm 100 \\
\mathrm{nM}\end{array}$ & 1 \\
\hline 398535 & & $2 \pm 1$ & $\begin{array}{c}400 \pm 100 \\
\mathrm{nM}\end{array}$ & $\begin{array}{c}400 \pm 200 \\
n M\end{array}$ & 0.3 \\
\hline LY-2-30 & & $>100$ & $>100$ & $>100$ & N/A \\
\hline $\begin{array}{c}\text { LY-2- } \\
\text { 173b-OTs }\end{array}$ & & $19 \pm 2$ & $7 \pm 1$ & $53 \pm 4$ & 4 \\
\hline
\end{tabular}

*Selectivity is defined as the ratio of $\mathrm{IC}_{50}$ on fibroblast over the $\mathrm{IC}_{50}$ average in the two cancer cell lines. 
depending on the criteria of the connectivity search, completely novel structural features and new molecular platforms could be identified, but loss of activity may be expected. These two approaches are therefore highly complementary.

\subsubsection{Comparison of Two Similarity Search Methods}

Generally speaking, the connectivity similarity search gave us more diverse structure scaffolds that are bioactive than did the shape similarity search. For example, I got eight bioactive compounds in the first round of whole-library connectivity similarity search and four bioactive compounds in the further search. I obtained highly varied scaffolds such as compound UC193691, in which I found the lead compound trimethoxyphenyl ring replaced by an isothiourea group; in compound UC98514, I found the lead compound phenyl right replaced by the amidine group. But in the shape similarity search, I only got two bioactive compounds, and they both shared very close features with the lead compound. On the other hand, the shape similarity search gave us more bioactive compounds than did the connectivity search. The two hits from the shape similarity search had $\mathrm{IC}_{50}$ values on melanoma cell lines in the nanomolar range, while hits from the connectivity search were mostly in the micromolar range. The reason for this difference was largely to the result of the inherent algorithm difference of these two screening approaches.

We did not choose receptor-based virtual screening because we did not confirm the target for this new class compounds at the initial stage. Later mechanism of action studies revealed that the target is the tubulin colchicine binding site.

\subsection{Conclusions}

Using both connectivity- and shape similarity-based virtual screening against a large database, I identified 14 new molecules from the UC compound library that are active against melanoma cells. These molecules provide several new functional groups and structure features compared with the lead structure. In summary, the novel pharmacophoric elements identified through these virtual screening exercises include a) a terminal basic amine group can improve activity and selectivity; b) a properly constructed amide linker between the five-member ring and the trimethoxyphenyl ring is beneficial to bioactivity; c) the thiazole ring in the lead compound is not necessary for bioactivity, and it can be replaced with an N-methylene hydrazine linker; d) an isothiourea group can replace the lead compound trimethoxyphenyl ring; and e) an amidine group can replace the phenyl ring of the lead compound.

This information is helpful for understanding the structure-activity relationship and for further improving compound activity. While the shape similarity search did not provide diverse active structures, the active compounds identified were generally much more active than those identified by the connectivity search. Combining both connectivity and shape similarity search techniques was complementary for our ligand- 
based drug discovery efforts. Further modification of the lead compound is in progress based on the information obtained from these virtual screenings. 


\section{CHAPTER 4. DISCOVERY AND BIO-EVALUATION OF 4-SUBSTITUTED METHOXYBENZOYL-ARYL-THIAZOLE AS NOVEL ANTI-MELANOMA AGENTS*}

\subsection{Introduction}

Microtubule is composed of $\alpha$ - and $\beta$-tubulin dimmer, which plays an important role in cell mitosis, motility and organelle distribution (117). The formation of microtubule from tubulin is a dynamic process that involves the polymerization and depolymerization of $\alpha$ - and $\beta$-heterodimer. These dynamic behaviors are crucial to cell mitosis, which is the process of chromosomal division to form new cells (118). Interference with tubulin-microtubule dynamics may be one of the attractive potential strategies in developing an anticancer drug $(119,120)$. There are some antitubulin agents developed with great potency, which may achieve low nano-molar range in the inhibition of cell proliferation by inducing cell cycle to be arrested in $\mathrm{G} 2 / \mathrm{M}$ phase and subsequently the inhibition of cell division $(121,122)$.

Tubulin heterodimer has three major known pharmacologic sites, including taxane, vinca, and colchicine site. Vinca and colchicine domain ligands destabilize and inhibit microtubule formation. Taxane-site agents stabilize and promote the formation of microtubules, having the opposite effects of vinca and colchicine domain ligands. Some of the antitubulin agents have been successfully developed for clinical use, such as paclitaxel on taxane domain, and vincristine, vinorelbine, vinblastine on vinca domain. However, they are substrates of P-glycoprotein and have drug resistance issues $(123,124)$. Although no clinical application colchicine-domain binding drugs yet, there are some agents which are on the phase I or II stage of development $(125,126)$. To address the drug resistance of antitubulin agents for each binding-domain, many new generation antitubulin agents are developed to overcome the issue caused by over expression of Pglycoprotein and/or the MDR1 (127-130).

I have been actively engaged in searching novel anticancer agents that target on tubulin. More recently, a series of 4-Substituted Methoxylbenzoyl-Aryl-Thiazole (SMART) has been developed in our laboratory (100). This series compounds was developed from 2-aryl-thiazolidine-4-carboxylic acid amides (ATCAA) which I discussed in chapter 2. SAR studies of ATCAA indicated that replacement of the lipid chain with a bulky aromatic ring in the 4-amide position of ATCAA attached to the thiazolidine ring still kept the anti-proliferative activity. This finding afforded us a new point to replace the fatty amide chain with a number of aromatic groups, which would maintain the cytotoxicity. With further investigation of ATCAA analogues, structure modifications were made on thiazolidine ring and 4-carboxylic amide linker. Thus, substituted methoxylbenzoyl-aryl-thiazole (SMART) compounds were discovered.

*Portions of this chapter adapted with permission from Lu Y, Li CM, Wang Z, et al. Discovery of 4substituted methoxybenzoyl-aryl-thiazole as novel anticancer agents: synthesis, biological evaluation, and structure-activity relationships. J Med Chem 2009;52:1701-11. 
The general structure of SMART agents with as showed in Figure 4-1. The SMART agents have a structure containing three conjugated aromatic rings (A, B and $\mathrm{C}$ rings, respectively) with a ketone linkage between $\mathrm{B}$ and $\mathrm{C}$ rings. Thiazole was introduced in $\mathrm{B}$ ring instead of thiazolidine ring in ATCAA. The linker between B and C rings was modified from an amide to carbonyl group. The $\mathrm{C}$ ring was characterized by the presence of differently-substituted phenyl groups, in particular, the 3, 4, 5-trimethoxy substituted phenyl at $\mathrm{C}$ ring played an important role of antiproliferative activity against melanoma.

According to the study, I characterized the biologic effect using three representative SMART compounds: SMART-100, SMART-45, and SMART-211. SMART-100 and SMART-45 are the most potent SMART compounds in our laboratory; however, they exhibit poor solubility. SMART-211 was synthesized to increase solubility by adding a hydroxyl- group on the A-ring, and it still remained potent cytotoxicity with $\mathrm{IC}_{50}$ value of $120 \mathrm{nM}$ on several melanoma cell lines. I examined these SMARTs on cell proliferation in a variety of cancer cell lines including MDR-positive cell lines and summarized the SAR. I determined the binding site to tubulin, the ability of inhibition on tubulin polymerization. Together with Mr. Chien-ming Li, I determined pharmacokinetic parameters on mice, and developed a formulation for the improvement of the poor solubility of SMARTs. I also examined the anticancer efficacy using nude mice bearing human melanoma A375 xenograft.

\subsection{Materials and Methods}

\subsubsection{Compounds and Reagents}

All the SMART compounds were synthesized by Dr. Yan Lu. Paclitaxel, vinblastine, and colchicine were obtained from Sigma-Aldrich. Ethanol was obtained from Decon labs, Inc. Cremophor ELP was obtained from BASF, Inc. CellTiter 96® AQueous One Solution Reagent was obtained from Promega.

\subsubsection{Cell Culture and Cytotoxicity Assay}

The A375 and B16-F1 melanoma cancer cells were obtained from American Type Culture Collection (Manassas, VA). WM-164 cells were derived from patient metastatic melanoma tumors and were a gift from Dr. Meenhar Herlyn (Wistar Institute, Philadelphia, PA). Human Dermal Fibroblast (adult) cells (HDFa) were obtained from Cascade Biologics. This cell line was used as control to determine selectivity. The P-gp overexpressing multi-drug resistant cell line MDA-MB-435/LCC6MDRI and matching sensitive parent cell line were kindly provided by Dr. Robert Clarke (Georgetown University, Washington, DC). Cells were grown as monolayers by using standard cell culture conditions. All cells were cultured in DMEM (Mediatech, Inc., Manassas, VA), supplemented with 10\% FBS (Atlanta Biologicals, Lawrenceville, GA), 1\% 


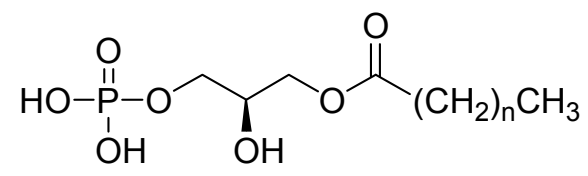

\section{LPA}

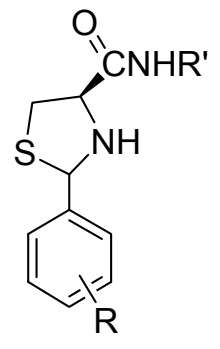

ATCAA

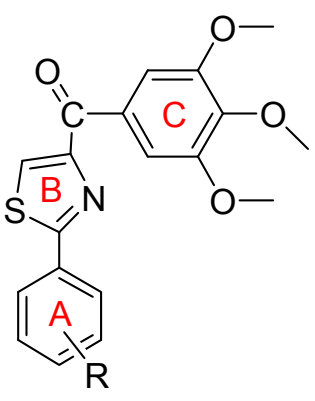

SMART

Fatty chain series, ATCAA-1:

$\mathrm{R}=4-\mathrm{NHCOCH}_{3}, \mathrm{R}^{\prime}=n-\mathrm{C}_{16} \mathrm{H}_{33}$

$\mathrm{R}=4-\mathrm{H}$, SMART-100

Aromatic ring series, ATCAA-2:

$\mathrm{R}=4-\mathrm{F}, \mathrm{SMART}-45$

$\mathrm{R}=4-\mathrm{NHCOCH}_{3}, \mathrm{R}^{\prime}=$

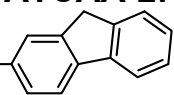

$\mathrm{R}=4-\mathrm{OH}, \mathrm{SMART}-211$

Figure 4-1. Structures of LPA, ATCAA and SMART. 
antibiotic/antimycotic mixture (Sigma-Aldrich, St. Louis, MO), and bovine insulin (5 $\mu \mathrm{g} / \mathrm{mL}$ ) (Sigma-Aldrich, St. Louis, MO).

Cultures were maintained at $37^{\circ} \mathrm{C}$ in a humidified atmosphere containing $5 \%$ $\mathrm{CO}_{2} .1000$ to 5000 cells were plated into each well of 96-well plates depending on growth rate and exposed to different concentrations of a test compound for $48 \mathrm{~h}$ in three to five replicates. Cell numbers at the end of the drug treatment were measured by the MTS assay. Absorbance was recorded at $490 \mathrm{~nm}$ in a BioTek EL800 96-well plate reader (BioTek Instruments, Inc., Winooski, VT). Percentages of cell survival versus drug concentrations were plotted and the $\mathrm{IC}_{50}$ (concentration that inhibited cell growth by $50 \%$ of untreated control) values were obtained by nonlinear regression analysis using GraphPad Prism (GraphPad Software, San Diego, CA).

\subsubsection{Cell Cycle Analysis}

Flow cytometry was performed to study cell cycle phase distribution. A375 cells were cultured in growth media until the confluence was about $70 \%$, and then cells were starved in $0.5 \%$ charcoal-stripped serum (CS-FBS) for $48 \mathrm{~h}$ before the experiment. A375 then were treated with $0,10,50,200$, and $1000 \mathrm{nM}$ of SMART-100, -45, and -211 for 24 $\mathrm{h}$ in growth media. The cellular DNA was stained with PBS containing $100 \mu \mathrm{g} / \mathrm{mL}$ propidium iodide, and $100 \mu \mathrm{g} / \mathrm{mL}$ RNase A. Flow cytometry assay was used to determine the distribution of a population of cells to different stages of cell cycle.

\subsubsection{In Vitro Microtubule Polymerization Assay}

Bovine brain tubulin (0.4 mg) (Cytoskeleton, Denver, CO) was mixed with 10 $\mu \mathrm{M}$ of test compound and incubated in $120 \mu \mathrm{L}$ of general tubulin buffer ( $80 \mathrm{mM}$ PIPES, $2.0 \mathrm{mM} \mathrm{MgCl}_{2}, 0.5 \mathrm{mM}$ EGTA, pH 6.9 and $1 \mathrm{mM} \mathrm{GTP}$ ). The absorbance of wavelength at $340 \mathrm{~nm}$ was monitored every 60 s for $20 \mathrm{~min}$ by the SYNERGY 4 Microplate Reader (Bio-Tek Instruments, Winooski, VT). The spectrophotometer was set at $37^{\circ} \mathrm{C}$ for tubulin polymerization.

\subsubsection{Tubulin Colchicine Competition Assay}

$[3 \mathrm{H}]$ colchicine competition-binding scintillation proximity assay (SPA) (Cytoskeleton Inc, Denver, CO) was used to evaluate SMART compounds that bind to the colchicine binding site on tubulin. Biotin-labeled tubulin $(0.5 \mu \mathrm{g})$ was mixed with $[3 \mathrm{H}]$ colchicine $(0.08 \mu \mathrm{M})$ (Perkinelmer LLC., Shelton, CT) and varies concentrations of SMARTs. Streptavidin-labeled SPA beads $(80 \mu \mathrm{g})$ (GE-healthcare Bio-Sciences Corp., Piscataway, NJ) are added, and co-incubated in $200 \mu \mathrm{L}$ binding buffer [ $80 \mathrm{mM}$ PIPES (pH 6.8), $1 \mathrm{mM}$ EGTA, $10 \%$ glycerol, $1 \mathrm{mM} \mathrm{MgCl} 2$, and $1 \mathrm{mM} \mathrm{GTP]} \mathrm{for} 45 \mathrm{~min}$ at $37^{\circ} \mathrm{C}$. $\mathrm{IC}_{50}$ values (concentrations that inhibited $50 \%$ of tritiated colchicine binding) were 
determined by nonlinear regression analysis with GraphPad Prism (GraphPad Software, San Diego,CA).

\subsubsection{Microtubule Imaging Using Fluorescence Microscope}

In order to get a visually appreciable proof of SMART compounds interacting with tubulin inside the cells, I examined microtubule arrangement in human melanoma WM-164 cells upon treatment with different compounds. I selected the Cellomics Cytoskeleton rearrangement kit (Thermo Scientific, Rockford, IL) which is widely used for such studies and followed the instructions from the manufacturer. WM-164 Melanoma cells were treated with each compound for $18 \mathrm{~h}$ in duplicate using a collagencoated 96-well plate (Becton Dickinson Labware, Bedford, MA). Then cells were fixed with 4\% paraformaldehyde (Thermo Scientific, Rockford, IL) and permeabillized using permeabilization buffer supply from the kit. Primary antibody for tubulin and fluorescence labeled secondary antibody were subsequently added to the cells. Cell nuclei were stained by DAPI. Whole Cell Stain Green was also applied to all cells. All images were acquired with an Olympus IX71 inverted fluorescence microscope (Olympus Corporation, Tokyo, Japan) with overlays from separate images of tubulin (red), nuclei (blue) and whole cells (green). For comparison, I also included paclitaxel, colchicine along with SMART compound.

\subsubsection{Pharmacokinetic Studies in Mice}

Male ICR mice 6 to 8 weeks of age were used to perform pharmacokinetic study. Drug solutions were dissolved in DMSO/PEG300 (80/20) and administrated by a single i.v. injection into the tail vein. For SMART-100, 5 and $15 \mathrm{mg} / \mathrm{kg}$ of body weight were administrated. For SMART-45, $15 \mathrm{mg} / \mathrm{kg}$ of body weight was administrated. Blood samples were obtained via cardiac puncture under isoflurane anesthesia at 2, 5, 15, and 30 min, 1, 2, 4, 8, 16, and $24 \mathrm{~h}$ after administration, using 3-4 animals per group. Sodium heparin ( $5 \mu \mathrm{L}$ of $1,000 \mathrm{USP}$ units/mL per sample) was used as an anticoagulant. Blood samples were collected on ice, and separated by centrifuging at $3000 \mathrm{~g}$ for $10 \mathrm{~min}$ to obtain plasma. All samples were stored at $-20^{\circ} \mathrm{C}$ until analysis.

Protein precipitation method was used for sample preparation. Plasma samples were thawed at room temperature. $150 \mu \mathrm{L}$ of acetonitrile (ACN) containing an internal standard, a SMART analog, was added to $100 \mu \mathrm{L}$ of plasma sample, and then was thoroughly vortexed for $15 \mathrm{~s}$. After centrifugation at $14,000 \mathrm{RPM}$ for $10 \mathrm{~min}$ at $4{ }^{\circ} \mathrm{C}$, the supernatant was transferred into high-performance liquid chromatography vials and directly used for LC-MS/MS analysis.

Plasma concentration-time data were analyzed by non-compartmental analysis using WinNonlin 5.2 (Pharsight Corporation, Mountain View, CA). The area under the plasma concentration-time curve from time 0 to infinity $\left(\mathrm{AUC}_{\mathrm{inf}}\right)$ was calculated by the trapezoidal rule with extrapolation to time infinity. The terminal half-life $\left(t_{1 / 2}\right)$ was 
calculated as $0.693 / \lambda_{z}$, where $\lambda_{z}$ is the terminal phase rate constant. The plasma clearance (CL) was calculated using the equation $\mathrm{CL}=$ Dose $_{\mathrm{iv}} / \mathrm{AUC}_{\mathrm{inf}}$, iv, where Dose $_{\text {iv }}$ and $\mathrm{AUC}_{\mathrm{inf} \text {, iv }}$ are the IV dose and corresponding area under the plasma concentration-time curve from time 0 to infinity, respectively. Volume of distribution based on terminal phase $\left(\mathrm{V}_{\mathrm{z}}\right)$ was calculated using $\mathrm{V}_{\mathrm{z}}=$ Dose $_{\text {iv }} /\left(\lambda_{\mathrm{z}}{ }^{*} \mathrm{AUC} \mathrm{C}_{\text {inf, iv }}\right)$. The peak plasma concentration $\left(\mathrm{C}_{\max }\right)$ and the time when it occurred $\left(t_{\max }\right)$ in the IP group were obtained by visual inspection of the plasma concentration-time curves. Clearance after IP $(\mathrm{CL} / \mathrm{F})$ was calculated as

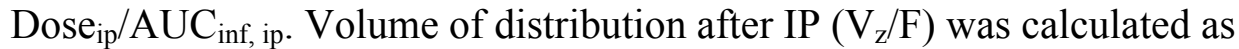
Dose $_{\text {ip }} /\left(\lambda_{z}{ }^{*} \mathrm{AUC}_{\text {inf, ip }}\right)$. IP bioavailability $(\mathrm{F})$ was calculated using $\mathrm{F}=\left(\mathrm{AUC} \mathrm{C}_{\mathrm{inf}, \mathrm{ip}}{ }^{*}\right.$

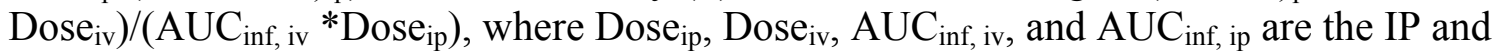
IV dose and the corresponding areas under the plasma concentration-time curves from time 0 to infinity, respectively.

\subsubsection{LC-MS/MS Method for Measuring SMART Compounds}

Sample solution $(10 \mu \mathrm{L})$ was injected into an Agilent series HPLC system (Agilent 1100 Series Agilent 1100 Chemstation, Agilent Technology Co, Ltd). SMART100 and SMART-45 were separated on a narrow-bore C4 column (Varian Inc, $2.1 \times 150$ $\mathrm{mm}, 5 \mu \mathrm{m}$, Palo Alto, CA). Gradient mode was used to achieve the separation of analytes using mixtures of mobile phase A (5\% acetonitrile containing $0.1 \%$ formic acid) and mobile phase B (95\% acetonitrile containing $0.1 \%$ formic acid) at a flow rate of 300 $\mu \mathrm{L} / \mathrm{min}$. Mobile phase A was used at $100 \%$ from 0 to 1 min followed by a linearly programmed gradient to $100 \%$ of mobile phase B within 3 min, $100 \%$ of mobile phase B was maintained for 1 min before a quick ramp to $100 \%$ mobile phase A. Mobile phase A was continued for another 8 min towards the end of analysis.

A triple-quadruple mass spectrometer, API Qtrap ${ }^{\mathrm{TM}}$ (Applied Biosystems/MDS SCIEX, Concord, Ontario, Canada), operating with a TurboIonSpray source was used. SMART-100 and SMART-45 were infused into the mass spectrometer at $1 \mathrm{mg} / \mathrm{L}$ and 10 $\mu \mathrm{L} / \mathrm{min}$ to optimize the instrument parameters used for LC-MS/MS. The Q1 full scan spectrums of SMART-100 and SMART-45 were first obtained to identify $[\mathrm{M}+\mathrm{H}]^{+}$ion as the precursor ion to generate product ion scans for selecting the most distinctive precursor-product ion pair. For the most sensitive quantitative analysis, the mass spectrometer was operated in the multiple reaction monitoring (MRM) mode, which includes $\mathrm{m} / \mathrm{z} 356.2 \rightarrow 188.2$ for SMART-100, 374.2 $\rightarrow 206.2$ for SMART-45, and m/z $434.0 \rightarrow 266.0$ for an internal standard, which is analog of SMART compound. Dwelling time was set to $100 \mathrm{~ms}$ for each analyst, and $50 \mathrm{~ms}$ for the internal standard. Detection limit is $0.2 \mathrm{ng} / \mathrm{mL}$ for SMART-45, -100 , and -211 .

The spraying needle voltage was set at $5000 \mathrm{~V}$ for positive mode. Curtain gas was set at 10; Gas 1 and gas 2 were set at 50. Collision-Assisted-Dissociation (CAD) gas at medium, and the source heater probe temperature at $500^{\circ} \mathrm{C}$. DP, EP, CXE were set at 16 , 10 , and $15 \mathrm{~V}$, respectively. Data acquisition and quantitative processing were accomplished using Analyst ${ }^{\mathrm{TM}}$ software, Ver. 4.0 (Applied Biosystems). 


\subsubsection{Antitumor Efficacy on A375 Xenografted Athymic Nude Mice}

A375 cells $\left(5 \times 10^{7} / \mathrm{mL}\right)$ were prepared in phenyl red free DMEM media containing 10\% FBS, and mixed with 1:1 of Matrigel (BD Biosciences, San Jose, CA). Tumors were established by injection $100 \mu \mathrm{L}$ of mixture $\left(2.5 \times 10^{6}\right.$ cells per animal, s.c. into low flank of mice) to 6-8-week-old male athymic nude mice. Treatments were initiated when tumor reached a mean group size of $150 \mathrm{~mm}^{3}$. Tumor volume $\left(\mathrm{mm}^{3}\right)$ was measured with calipers and determined by the formula, $\pi / 6 \times \mathrm{L} \times \mathrm{W}^{2}$, where length (L) and width (W) are in mm. The formulation of Tween80/Captex200 (80/20) were used to prepare SMART-100 (20 mg/kg), and SMART-45 (15 mg/kg). Mice were administrated by i.p. daily for 34 days (10 mice per group).

\subsection{Results and Discussion}

I studied the structure-activity relationship (SAR) in our laboratory and selected three potent SMARTs to perform the mechanism of action. SMART-100 has no substitution on the A-ring. SMART-45 and SMART-211 have fluorine (-F) and hydroxy $(-\mathrm{OH})$ on the A-ring, respectively. SMART-45 and -100 have poor solubility; however, SMART-211 is a water-soluble compound. The effects of SMART-100, -45, and -211 on the proliferation of melanoma cancer cells (B16-F1, A375, and WM-164) and normal fibroblast cells are summarized in Table 4-1. SMARTs inhibit the proliferation of prostate and melanoma cancer cells with $\mathrm{IC}_{50}$ values in the low nano-molar range. Overall, the potency of SMART-45 is similar to SMART-100, and greater than SMART211.

\subsubsection{SAR Studies}

\subsubsection{SAR of the B Ring}

Please see Figure 4-1 for the designation of A, B, and C ring of SMART compounds. In the previous ATCAA compounds, I found the thiazolidine ring, which contained a free NH at its 3-position, was important for cytotoxicity. Once the B ring thiazolidine moiety was replaced by a thiazoline ring, the antiproliferative activity decreased sharply from $600 \mathrm{nM}$ to over $50 \mu \mathrm{M}$ on WM-164 cell lines (83). The ATCAA1 fatty amide derivatives that were most effective against melanoma cell lines were examined and shown to have an $\mathrm{IC}_{50} 2 \mu \mathrm{M}$ (Table 4-2). Replacement of the long fatty chain with a certain aromatic bulky subsistent such as fluorene (ATCAA-2) showed inhibitory activity on both cancer cell lines $\left(\mathrm{IC}_{50}=2 \sim 4 \mu \mathrm{M}\right)$. The fluorene group in 4carboxylic amide position was also replaced by 3, 4, 5-trimethoxylphenyl group (2a and $2 \mathrm{~b})$, but the potency against both cancer cell lines was lost. The subsequent B ring modification from saturated thiazolidine compound 2 a to unsaturated thiazole 5 did not show any cytotoxicity against either cancer cell line tested. But thiazoline enantiomers $4 \mathrm{a}$ and $4 \mathrm{~b}(R$-isomer and $S$-isomer, showed similar antiproliferative activities) showed 
Table 4-1. Cytotoxic activity comparison of representative SMART compounds with other anticancer drugs.

\begin{tabular}{ccccc}
\hline \multirow{2}{*}{ Compound name } & \multicolumn{4}{c}{$\mathrm{IC}_{50} \pm \mathrm{SEM}(\mathrm{nM})(\mathrm{n}=3)$} \\
\cline { 2 - 5 } & $\mathrm{A} 375$ & $\mathrm{~B} 16-\mathrm{F} 1$ & $\mathrm{WM}-164$ & $\mathrm{HDFa}$ \\
\hline SMART-100 & $28 \pm 5$ & $55 \pm 5$ & $64 \pm 6$ & $40 \pm 5$ \\
SMART-45 & $29 \pm 11$ & $43 \pm 21$ & $41 \pm 6$ & $40 \pm 7$ \\
SMART-211 & $93 \pm 11$ & $113 \pm 6$ & $99 \pm 10$ & $289 \pm 92$ \\
Paclitaxel & $12 \pm 3$ & $17 \pm 2$ & $18 \pm 3$ & $12 \pm 3$ \\
Vinblastine & $1 \pm 0$ & $5 \pm 1$ & $1 \pm 0$ & $3 \pm 1$ \\
Colchicine & $20 \pm 3$ & $29 \pm 5$ & $10 \pm 2$ & $18 \pm 2$ \\
Bortezomib & $8 \pm 1$ & $24 \pm 2$ & $8 \pm 1$ & $26 \pm 2$ \\
\hline
\end{tabular}

Table 4-2. In vitro cytotoxic activity of modified ATCAA compounds.

\begin{tabular}{|c|c|c|c|c|c|c|}
\hline \multirow{2}{*}{$Y_{A}^{B-C}$} & \multirow[b]{2}{*}{ A ring } & \multirow[b]{2}{*}{$\mathrm{B}$ ring ${ }^{\mathrm{a}}$} & \multirow[b]{2}{*}{$\mathrm{C}$ ring $^{\mathrm{b}}$} & \multirow[b]{2}{*}{$\mathrm{X}$} & \multicolumn{2}{|c|}{$\mathrm{IC}_{50} \pm \operatorname{SEM}(\mu \mathrm{M})(\mathrm{n}=3)$} \\
\hline & & & & & B16-F1 & A375 \\
\hline ATCAA-1 & $p$-NHAc-Ph & TZD & $\mathrm{C}_{16} \mathrm{H}_{33}$ & $\mathrm{CONH}$ & $2 \pm 0$ & $2 \pm 0$ \\
\hline ATCAA-2 & $p$-NHAc-Ph & TZD & 9H-fluoren-1-yl & $\mathrm{CONH}$ & $4 \pm 0$ & $2 \pm 0$ \\
\hline $2 a$ & $\mathrm{Ph}$ & TZD & 3,4,5-trimethoxyl-Ph & $\mathrm{CONH}$ & $>100$ & $>100$ \\
\hline $2 b$ & $\begin{array}{l}3,4,5- \\
\text { trimethoxyPh }\end{array}$ & TZD & 3,4,5-trimethoxyl-Ph & $\mathrm{CONH}$ & $>100$ & $>100$ \\
\hline $4 \mathrm{a}(4 R)$ & $\mathrm{Ph}$ & TZL & 3,4,5-trimethoxyl-Ph & $\mathrm{CONH}$ & $38 \pm 3$ & $23 \pm 2$ \\
\hline $4 \mathrm{~b}(4 S)$ & $\mathrm{Ph}$ & TZL & 3,4,5-trimethoxyl-Ph & $\mathrm{CONH}$ & $30 \pm 3$ & $14 \pm 1$ \\
\hline 5 & $\mathrm{Ph}$ & $\mathrm{TZ}$ & 3,4,5-trimethoxyl-Ph & $\mathrm{CONH}$ & $>100$ & $>100$ \\
\hline $8 f$ & $\mathrm{Ph}$ & $\mathrm{TZ}$ & 3,4,5-trimethoxyl-Ph & $\mathrm{CO}$ & $55 \pm 5 \mathrm{nM}$ & $28 \pm 5 \mathrm{nM}$ \\
\hline Colchicine & & & & & $29 \pm 5 \mathrm{nM}$ & $20 \pm 3 \mathrm{nM}$ \\
\hline
\end{tabular}

${ }^{\mathrm{a}} \mathrm{TZD}=$ thiazolidine, $\mathrm{TZL}=$ thiazoline, $\mathrm{TZ}=$ thiazole

${ }^{b}$ For ATCAA-1, C position contains a lipid chain. 
improved activity $\left(\mathrm{IC}_{50}=14 \sim 38 \mu \mathrm{M}\right)$ compared with $2 \mathrm{a}, 2 \mathrm{~b}$ and 5 . When the amide $\mathrm{CONH}$ linkage between $\mathrm{B}$ ring and $\mathrm{C}$ ring was replaced by a carbonyl linker, the mixtures of thiazoline/thiazole ketone $8 \mathrm{f}$ were obtained instead of desired thiazoline ketone, because the auto-dehydrogenation between thiazoline and thiazole occurred. Surprisingly, introduction of the carbonyl group linker and thiazole led to a significant enhancement of growth inhibition of examined cancer cell lines with a low nanomolar level (8f, $\mathrm{IC}_{50}=21 \sim 71 \mathrm{nM}$ ), which is comparable to the natural anticancer agent colchicine. Thus a series of the SMART compounds with B as a thiazole ring were designed and synthesized based on the discovery of $8 \mathrm{f}$ and their anticancer activity was evaluated against melanoma.

\subsubsection{SAR of the C Ring}

We started our investigation of the C position of the SMART by introducing different substituted phenyls or alkyl chain. Variation of the phenyl substituents has a remarkable change in effect on potency. The in vitro assay as shown in Table 4-3 gave us an interesting result but only 3, 4, 5-trimethoxylphenyl in C ring (8f) showed excellent inhibition against all cancer cells $\left(\mathrm{IC}_{50}=28 \sim 55 \mathrm{nM}\right)$. Compound $8 \mathrm{~g}$, with a 3, 5dimethoxyphenyl group, showed 6-fold average cytotoxicity lower than $8 \mathrm{f}$. Modifications of $8 \mathrm{f}$ by removal of one methoxy at meta-position (8e) or two methoxy groups $(8 \mathrm{~b}, 8 \mathrm{c}$ and $8 \mathrm{~d}$ ) from $8 \mathrm{f}$ led to a dramatic loss in activity $\left(\mathrm{IC}_{50}>20 \mu \mathrm{M}\right)$. Although orthosubstituted monomethoxy compound $8 \mathrm{~d}$ exhibited weak activity against a certain cell lines compared with meta-/para-MeO substituted $8 \mathrm{c} / 8 \mathrm{~b}$ and dimethoxyphenyl compound $8 \mathrm{e}$, none of them showed significant potency in inhibition compared with $8 \mathrm{f}$. Similar trends were also seen in $8 \mathrm{~h}$ and $8 \mathrm{j}$ with 2-fluorophenyl and hexadecyl in $\mathrm{C}$ ring modifications.

\subsubsection{SAR of the A Ring}

In SAR studies of the ATCAA compounds, I found that the electronic properties of substituents of the phenyl ring in the 2-positioin of the thiazolidine ring strongly affected the anticancer activity in ATCAA compounds - electron-withdrawing groups (EWG) on 2-phenyl gave higher activities than those with electron-donating groups (EDG). Dr. Lu also introduced different para-substituted EWG and EDG on A phenyl ring of the SMART molecules. From the $\mathrm{IC}_{50}$ value against these cancer cell lines, electronic effects of A ring phenyl substituents did not show clear influence on antiproliferative activity. Introduction of a weak EWG (4-F in $\left.8 \mathrm{n}, \mathrm{IC}_{50} \mathrm{~s}: 33 \sim 43 \mathrm{nM}\right)$ or weak EDG (4- $\mathrm{CH}_{3}$ in $8 \mathrm{k}, \mathrm{IC}_{50} \mathrm{~s}: 11 \sim 21 \mathrm{nM}$ ), both increased the potency compared with $8 \mathrm{f}$ (see Table 4-4). The replacement of para-position with strong EWG such as $\mathrm{NO}_{2}(8 \mathrm{p})$, $\mathrm{CN}(8 \mathrm{q}), \mathrm{CF}_{3}(8 \mathrm{t})$ or introducing strong EDG (3, 4-dimethoxy) to A phenyl ring (8o) exhibited comparable antiproliferative activity.

To compare the effects of ortho-, meta- and para- substitutions, a fluoro atom was introduced to different positions of A phenyl ring (81, $8 \mathrm{~m}$ and $8 \mathrm{n}$ ). The various $o^{-}, m^{-}, p^{-}$ 
Table 4-3. In vitro cytotoxic activity of SMART compounds with $\mathrm{C}$ rings modification.

\begin{tabular}{rrccc}
\hline \multirow{2}{*}{ Compounds } & C Ring & \multicolumn{2}{c}{$\mathrm{IC}_{50} \pm \operatorname{SEM}(\mu \mathrm{M})(\mathrm{n}=3)$} \\
\cline { 3 - 4 } & $\mathbf{8 a}$ & $\mathrm{Ph}$ & $\mathrm{B} 16-\mathrm{F} 1$ & $\mathrm{~A} 375$ \\
$\mathbf{8 b}$ & 4-Methoxy-Ph & $>100$ & $>100$ \\
$\mathbf{8 c}$ & 3-Methoxy-Ph & $>100$ & $>100$ \\
$\mathbf{8 d}$ & 2-Methoxy-Ph & $59 \pm 21$ & $70 \pm 33$ \\
$\mathbf{8 e}$ & 3, 4-Dimethoxy-Ph & $>100$ & $>100$ \\
$\mathbf{8 f}$ & 3,4,5-Trimethoxy-Ph & $55 \pm 5 \mathrm{nM}$ & $28 \pm 5 \mathrm{nM}$ \\
$\mathbf{8 g}$ & 3, 5-Dimethoxy-Ph & $350 \pm 20 \mathrm{nM}$ & $170 \pm 10 \mathrm{nM}$ \\
$\mathbf{8 h}$ & 2-Fluoro-Ph & $>100$ & $>100$ \\
$\mathbf{8 j}$ & Hexadecyl & $19 \pm 18$ & $16 \pm 15$ \\
\hline
\end{tabular}

${ }^{\mathrm{a}}$ Compound $8 \mathrm{j}$ has a lipid chain at $\mathrm{C}$ ring position.

Table 4-4. In vitro cytotoxic activity of SMART compounds with A rings modification.

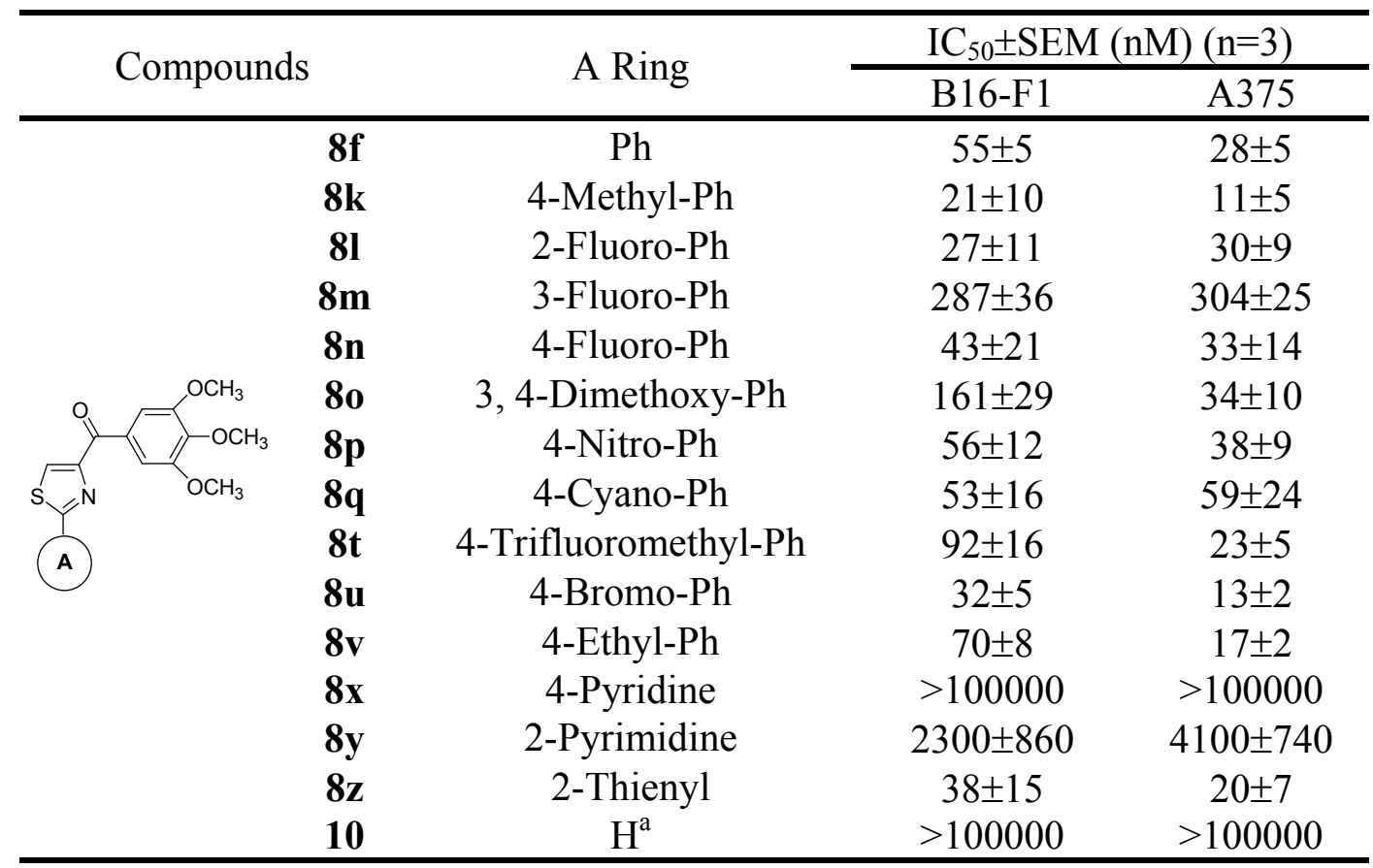

${ }^{\mathrm{a}}$ Compound 10 has a proton at A ring position. 
substituents did not exhibit equal activities. $o$-fluoro substituted 81 showed the lowest $\mathrm{IC}_{50} \mathrm{~s}(27 \sim 30 \mathrm{nM})$ against melanoma cells. $8 \mathrm{n}$ has similar average $\mathrm{IC}_{50} \mathrm{~s}(33 \sim 43 \mathrm{nM})$ against melanoma compared with 81 . Meta-substituted compound $8 \mathrm{~m}$ showed lowest activity on melanoma cells ( $\left.\mathrm{IC}_{50} \mathrm{~s}: 287 \sim 304 \mathrm{nM}\right)$.

Turning to the effects of steric hindrance group on the A phenyl ring substituents, I found that $p$-bromo $\left(8 \mathrm{u}, \mathrm{IC}_{50} \mathrm{~s}: 13 \sim 32 \mathrm{nM}\right)$ caused a slightly decrease in antiproliferative activity relative to $p$-fluoro position $\left(8 \mathrm{n}, \mathrm{IC}_{50} \mathrm{~s}: 6 \sim 12 \mathrm{nM}\right)$. Reduced activity against both cancer cell lines is obvious when $p$-methyl $\left(8 \mathrm{k}, \mathrm{IC}_{50} \mathrm{~s}: 11 \sim 21 \mathrm{nM}\right)$ was replaced with a $p$ ethyl group (8v, $\left.\mathrm{IC}_{50} \mathrm{~s}: 17 \sim 70 \mathrm{nM}\right)$.

To investigate if phenyl played an essential role at A ring in cytotoxicity, Dr. Lu also removed phenyl at 2-thiazole position and compound 10 was obtained. This modification caused a total loss of activity compared with $8 \mathrm{f}$. The replacement of the A ring by pyridine (compound $8 \mathrm{x}$ ) had the same effect. Moreover, substituting 2-pyrimidine in A ring (compound 8y) also caused a significant loss of activity ( $\mathrm{IC}_{50} \mathrm{~S}: 2 \sim 4 \mu \mathrm{M}$ ). However, introducing the thiophene replacement of phenyl (8z) into A position slightly improved the potency $\left(\mathrm{IC}_{50} \mathrm{~s}: 20 \sim 38 \mathrm{nM}\right)$ compared to $8 \mathrm{f}\left(\mathrm{IC}_{50} \mathrm{~s}: 28 \sim 55 \mathrm{nM}\right)$.

\subsubsection{SAR of Pharmaceutically Acceptable Salt Groups to the SMARTs}

Most of the SMART compounds have good solubility in organic solvents such as $\mathrm{CHCl}_{3}, \mathrm{CH}_{2} \mathrm{Cl}_{2}$ and DMSO. But they show poor water-solubility. Dr. Lu designed and synthesized water-soluble salts of the SMART by introducing a hydrophilic group such as $\mathrm{NH}_{2}(8 \mathrm{w})$ and $\mathrm{COOH}(8 \mathrm{r})$ into A ring and generated the $\mathrm{HCl}$ or sodium salt. Another modification is replacing $\mathrm{A} / \mathrm{C}$ rings in $8 \mathrm{a}$ with pyridine $(8 \mathrm{i}, 8 \mathrm{x}, 8 \mathrm{y})$ or pyrimidine rings, which could also be converted into $\mathrm{HCl}$ salts. These modifications reduced the calculated $\log \mathrm{P}$ values $(\log \mathrm{P}=3 \sim 4)$ compared with $8 \mathrm{a}$ and $8 \mathrm{f}(\log \mathrm{P}=4$, See Table 4-5). Introducing $p$-amino to A phenyl $(8 \mathrm{w})$ is the only case to increase the antiproliferative activity $(\mathrm{HCl}$ salt, $\mathrm{IC}_{50} \mathrm{~s}: 11 \sim 29 \mathrm{nM}$ ) compared with $8 \mathrm{f}$ against all cell lines. Although replacing phenyl with pyrimidine (8y) kept partial activity against both cancer cells, the potency range was markedly reduced from nano-molar to micromolar compared with 8f. Unfortunately introducing $\mathrm{COOH}$ to para- phenyl $\mathrm{A}$ ring and pyridine to $\mathrm{A}$ or $\mathrm{C}$ rings (8i, 8r, 8x) all resulted in the total loss of the anticancer activity. A total loss of potency was seen in the methyl ester 8s of acid 8r against both cancer cell lines. Demethylation of compound $8 \mathrm{f}$ afforded water soluble 3, 4, 5-trihydroxyphenyl at $\mathrm{C}$ ring compound 11, but this demethylation results in complete loss of antiproliferative activity against all tested cancer cells, which also points out the importance of 3, 4, 5-trimethoxyphenyl at C position.

\subsubsection{SAR Summary}

The general SAR of SMART compounds on melanoma cells was summarized in Figure 4-2. Present SAR studies revealed that 3, 4, 5- trimethoxyphenyl was the essential 
Table 4-5. In vitro cytotoxic activity of SMART compounds containing hydrophilic group.

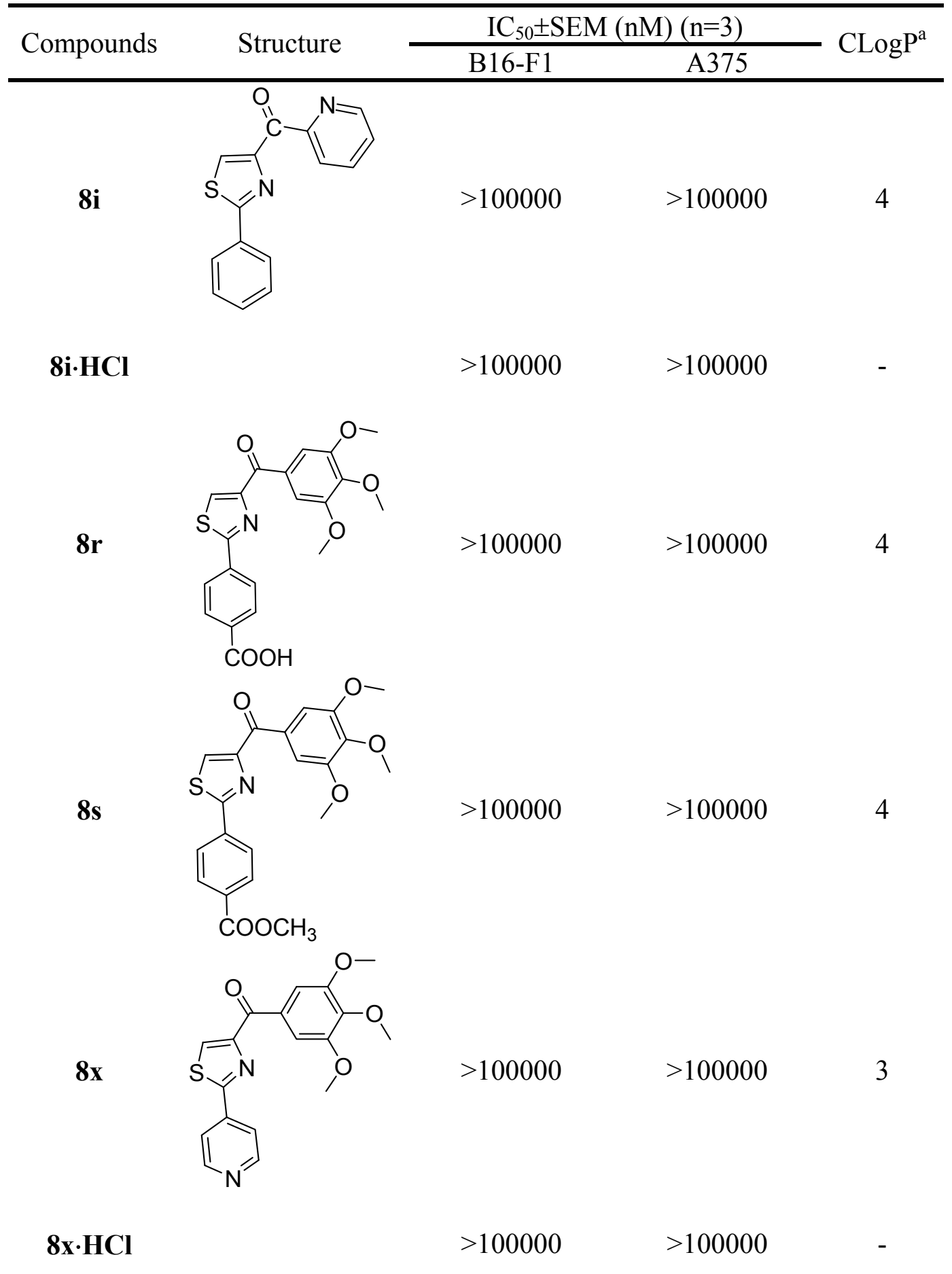


Table 4-5. (continued)

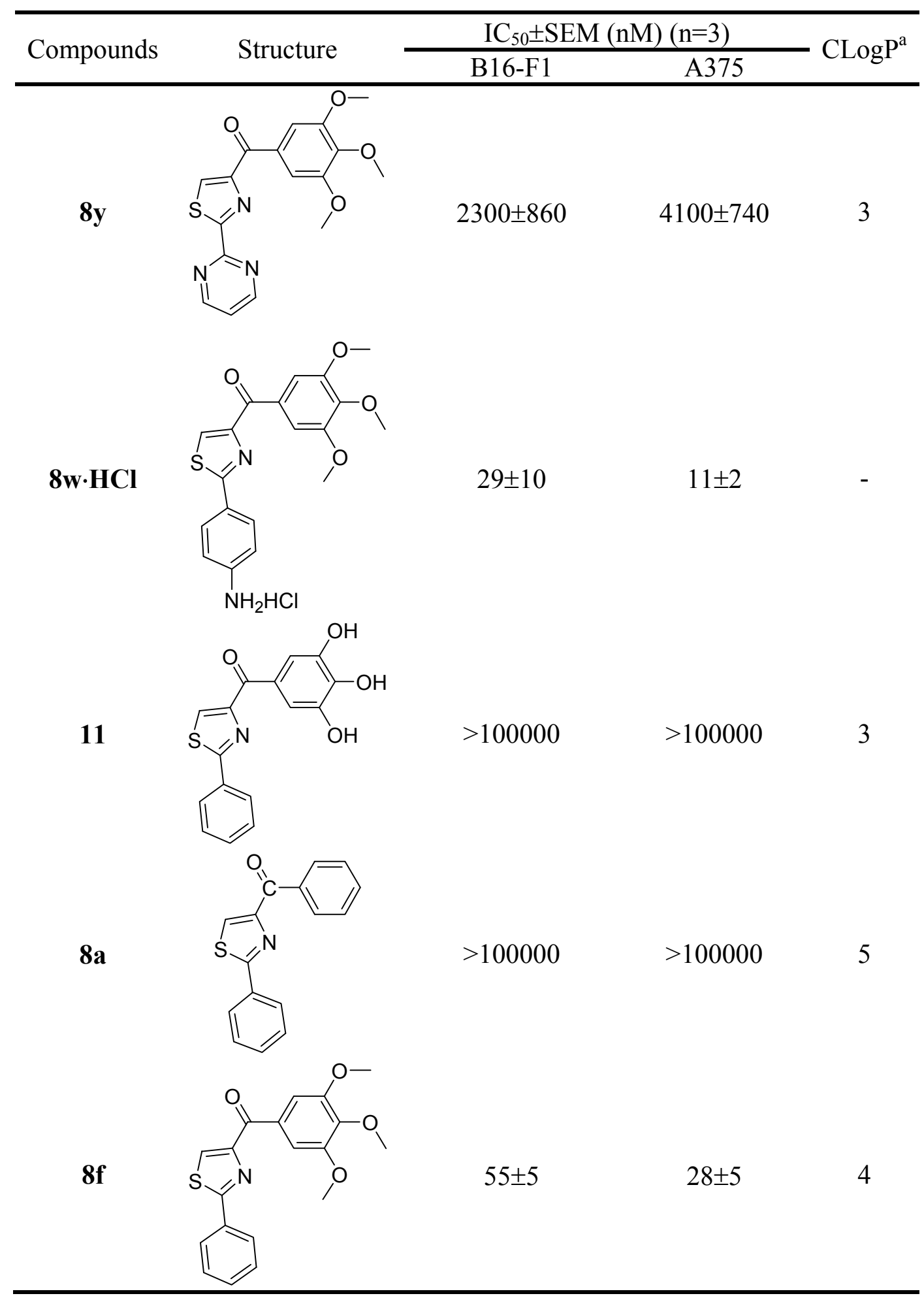

${ }^{a}$ Calculated LogP data using Chemoffice 2005, Chemdraw Ultra 9.0 software.

${ }^{\mathrm{b}} \mathrm{LogP}$ value were calculated based on free base. 
(d). Ketone $\mathrm{C}=\mathrm{O}$ linkage significantly improved the potency compared with CONH linker.

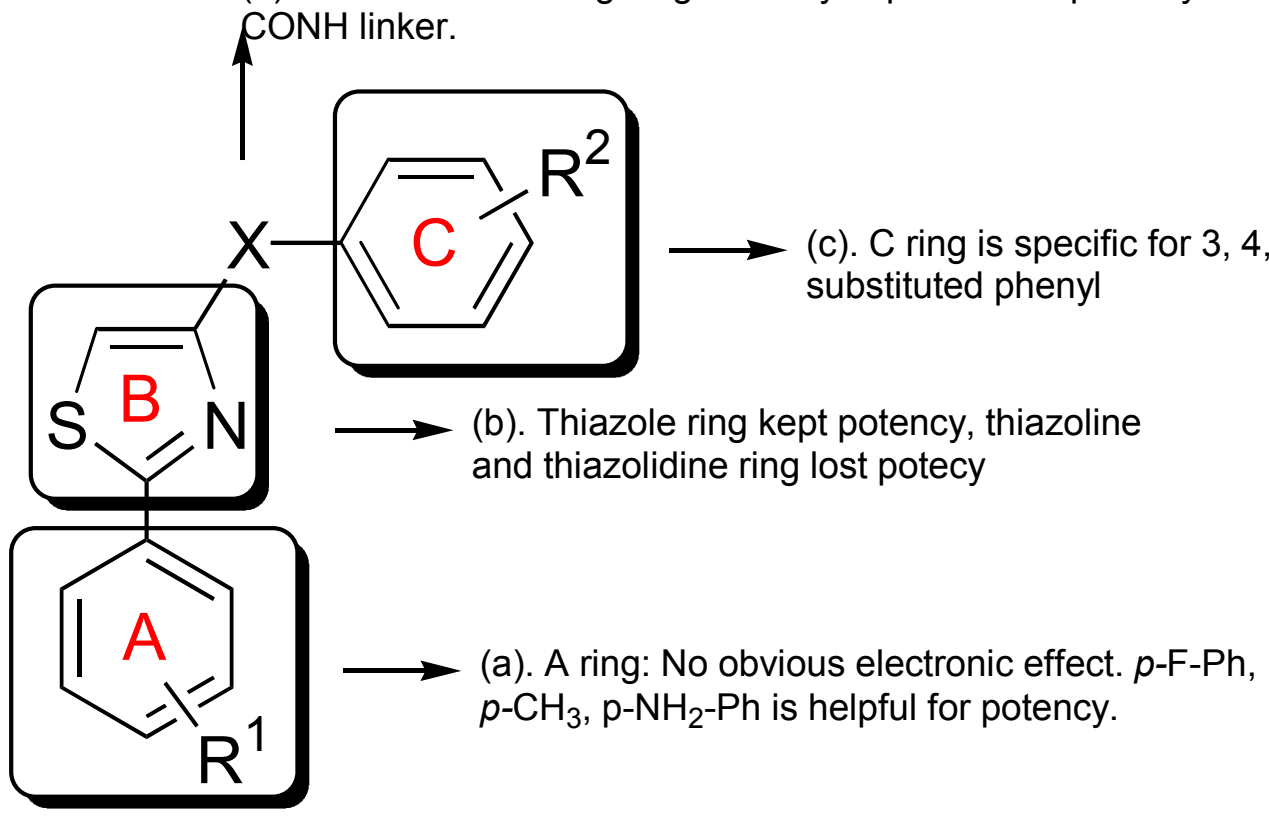

Figure 4-2. SAR summary of the SMART compounds. 
group in the $\mathrm{C}$ ring to keep excellent antitumor potency. $p$-Fluoro, $p-\mathrm{NH}_{2}$ and $p-\mathrm{CH}_{3}$ substituents in A ring will increase the activity, with no clear difference in effect on activity between EWG and EDG when A are substituted phenyl rings. The carbonyl linkage between $\mathrm{B}$ ring and $\mathrm{C}$ ring played an important role for the high potency.

\subsubsection{SMARTs Overcome Multi-Drug Resistance on Drug Resistant Melanoma Cells}

MDA-MB-435/LCC6MDRI cells over-express P-gp, and were used to test whether SMARTs were substrates of P-glycoprotein comparing to its parental cell line, MDA-MB-435. I demonstrated that paclitaxel, vinblastine, and colchicine were more resistant against MDR-positive cells than those with the parent sensitive cells. Please see Figure 4-3 for selected dose-response curves and Table 4-6 for summary of results. The resistance indexes were 69.3, 27.5, and 65.8 respectively. In the meantime, SMARTs did not show significantly difference on those two cell lines. The resistance indexes were around 1. This strongly suggested that SMARTs were not substrates for P-glycoprotein.

\subsubsection{SMARTs Target Tubulin on Colchicine Binding Site}

The strategy I used here to find out the cellular target of SMART compounds is the same as I described for ABI compounds in Chapter 5. I first did a cell cycle analysis for A375 cells after treatment with SMART-100, -45 , and $-211(0,10,50,200$, and 1000 $\mathrm{nM}$ ) for $24 \mathrm{~h}$ prior to staining with propidium iodide and cell cycle analysis. The proportion of cells in G2/M phase increased when treated with $50 \mathrm{nM}$ of SMART-100 and -45 , and SMART-211 showed the effect until the concentration was $200 \mathrm{nM}$. Some representative cell cycle distribution graphs were shown in Figure 4-4. Quantified representation of all results was shown in Figure 4-5. SMART-100 and SMART-45 had no effect on cell cycle distribution below $50 \mathrm{nM}$. About $16 \%$ of untreated cells were observed in $\mathrm{G} 2 / \mathrm{M}$ phase, whereas the cells treated with more than $50 \mathrm{nM}$ of colchicine or SMART compounds showed a greater proportion of cells in $\mathrm{G} 2 / \mathrm{M}$ phase. The increase in $\mathrm{G} 2 / \mathrm{M}$ phase cells was accompanied by a decrease in G1 populations, relative to control.

In vitro tubulin polymerization assay was used to identify the target of SMART compound on tubulin. This experiment was performed by my colleague Mr. Chien-Ming $\mathrm{Li}$. He demonstrated that SMART-100 inhibited tubulin polymerization in a doseresponse manner in the previous study (15). In the current study, $10 \mu \mathrm{M}$ of SMARTs were used to examine the inhibition of tubulin polymerization. In Figure 4-6, all three SMART compounds inhibited tubulin polymerization, indicating that SMARTs did target on tubulin. SMART-100 showed similar inhibitory ability as SMART-45, and greater than SMART-211. In vitro tubulin polymerization could be blocked by SMARTs, and the ability of inhibition on tubulin polymerization was also related to their $\mathrm{IC}_{50}$ values on cell anti-proliferation. $10 \mu \mathrm{M}$ of substrates were examined in this study because high concentration of tubulin $(4 \mathrm{mg} / \mathrm{mL})$ was used to form microtubule. 

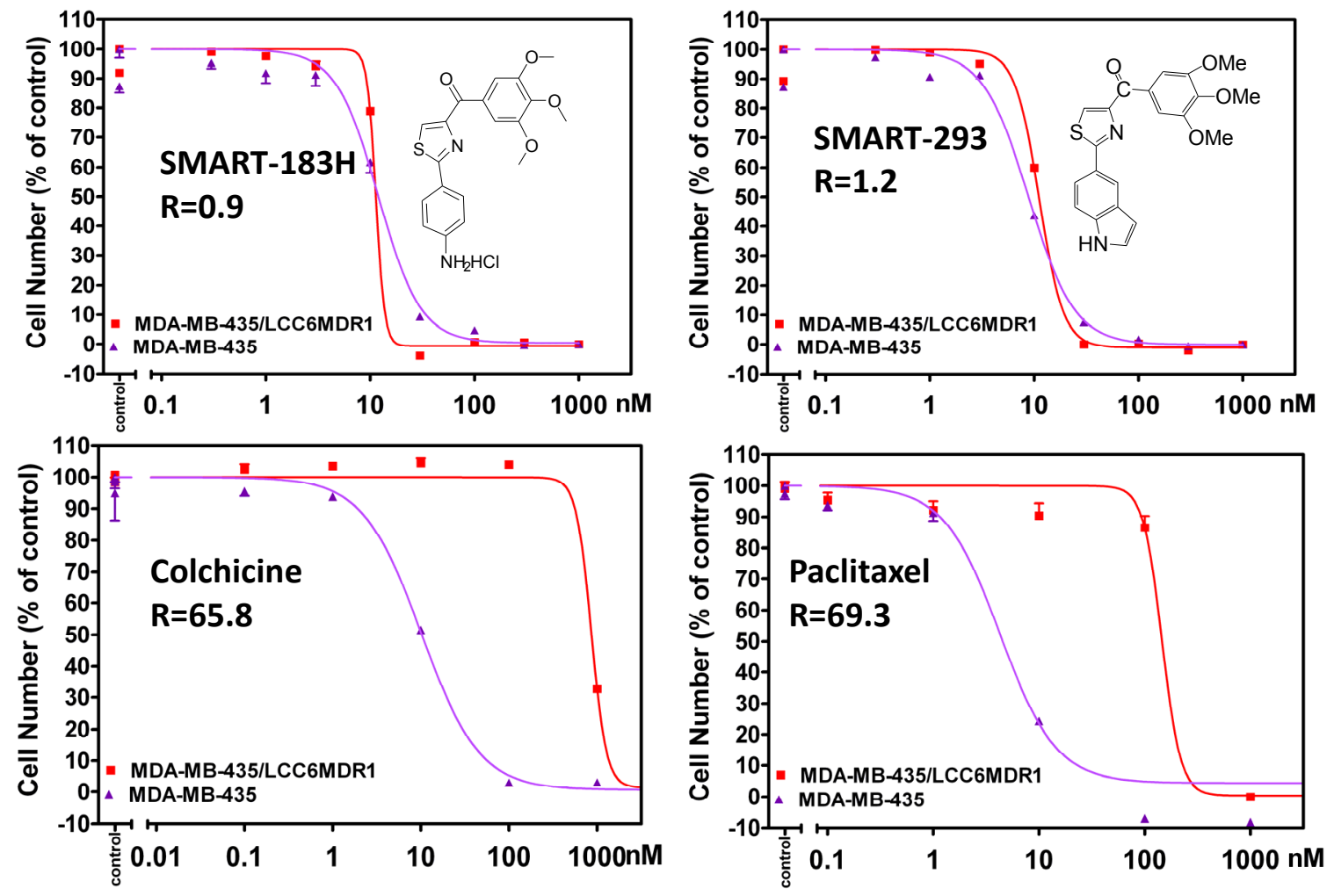

Figure 4-3. Dose response curves showed SMART compounds overcome multi-drug resistant for melanoma compared with colchicine and paclitaxel.

$\mathrm{R}$ : resistance index, this value were calculated by dividing $\mathrm{IC}_{50}$ values on multi-drug resistant cell line MDA-MB-435/LCC6MDR1 by $\mathrm{IC}_{50}$ values on the matching sensitive parent cell line MDA-MB-435. 
Table 4-6. SMART compounds showed equal potency against multi-drug resistant melanoma cells and parent sensitive cells.

\begin{tabular}{|c|c|c|c|c|}
\hline \multirow[b]{2}{*}{ Compounds } & \multirow[b]{2}{*}{ Structure } & \multicolumn{2}{|c|}{$\mathrm{IC}_{50} \pm \operatorname{SEM}(\mathrm{nM})(\mathrm{n}=3)$} & \multirow{2}{*}{$\begin{array}{l}\text { Resistance } \\
\text { index }(\mathrm{R}) *\end{array}$} \\
\hline & & $\begin{array}{l}\text { MDA-MB- } \\
435\end{array}$ & $\begin{array}{l}\text { MDA-MB-435 } \\
\text { /LCC6MDRI }\end{array}$ & \\
\hline SMART-293 & & $9 \pm 1$ & $11 \pm 2$ & 1 \\
\hline SMART-183H & & $12 \pm 2$ & $11 \pm 1$ & 1 \\
\hline Colchicine & & $10 \pm 1$ & $658 \pm 50$ & 66 \\
\hline Paclitaxel & & $4 \pm 1$ & $277 \pm 41$ & 69 \\
\hline Vinblastine & & $\begin{array}{c}400 \pm 100 \\
\text { pM }\end{array}$ & $11 \pm 1$ & 28 \\
\hline
\end{tabular}

*Resistance indexes were calculated by dividing $\mathrm{IC}_{50}$ values on multi-drug resistant cell line MDA-MB-435/LCC6MDR1 by $\mathrm{IC}_{50}$ values on the matching sensitive parent cell line MDA-MB-435. 

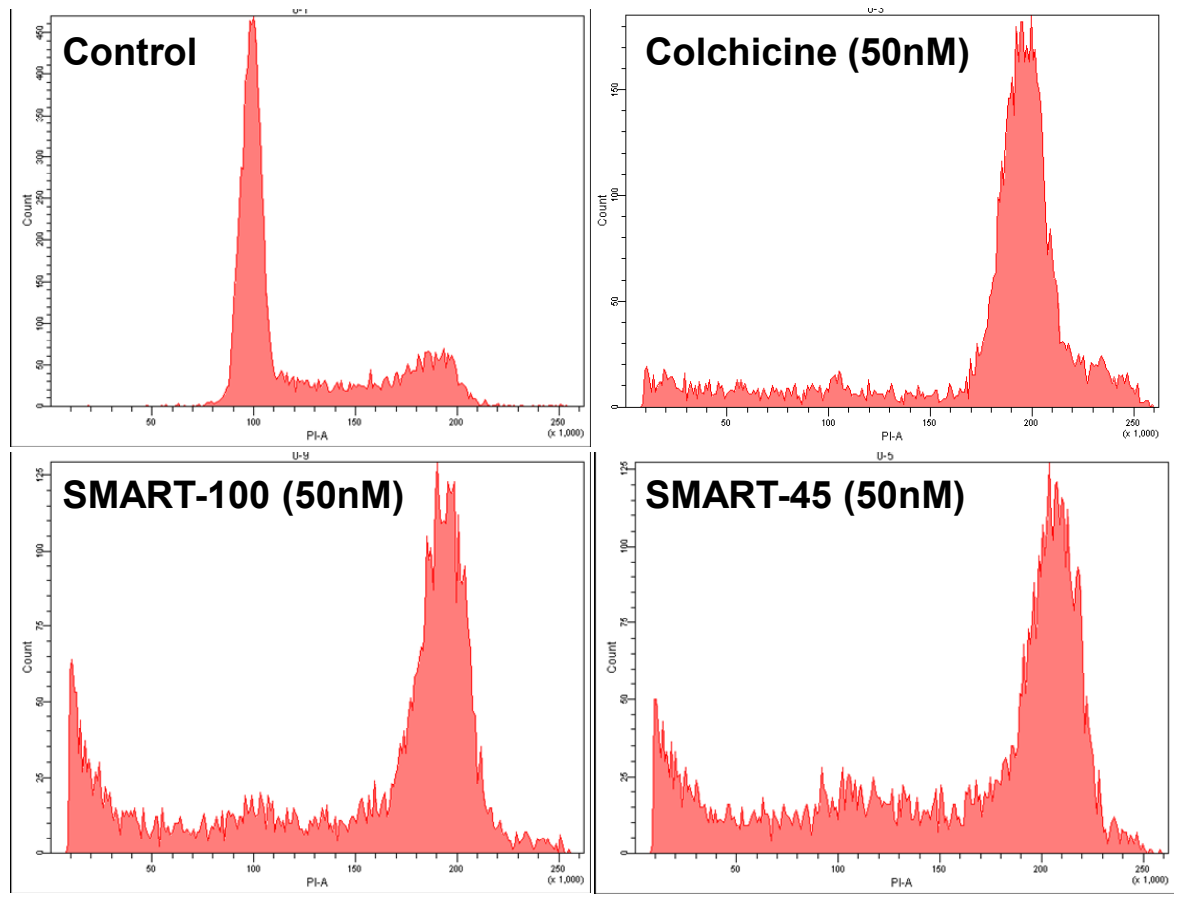

Figure 4-4. Cell cycle analysis showed SMART compounds and colchicine arrested A375 cells in G2/M phase after incubation for $24 \mathrm{~h}$.

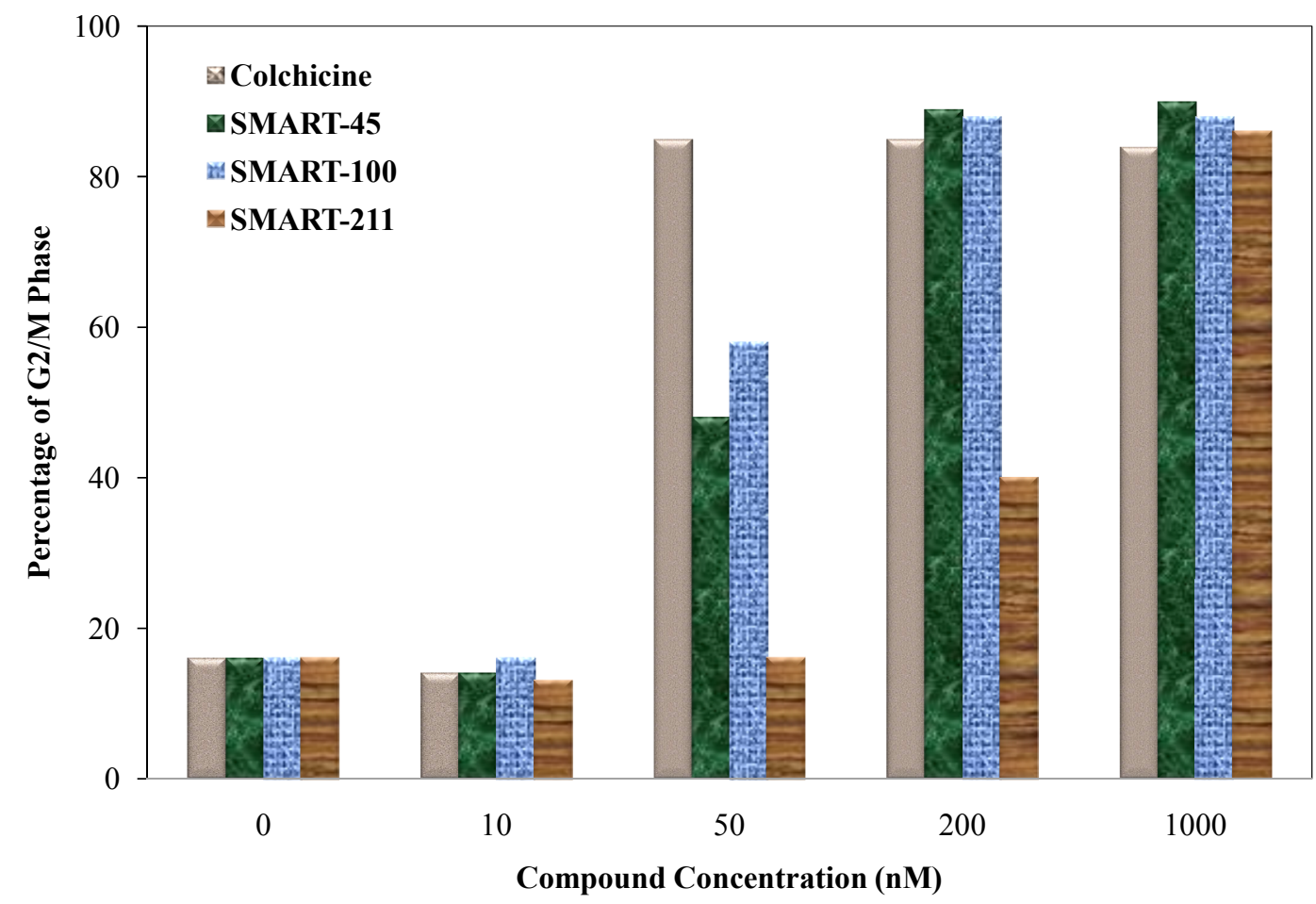

Figure 4-5. Quantified cell cycle analysis results showed SMART compounds and colchicine arrested A375 cells into G2/M phase in a dose dependent manner. 


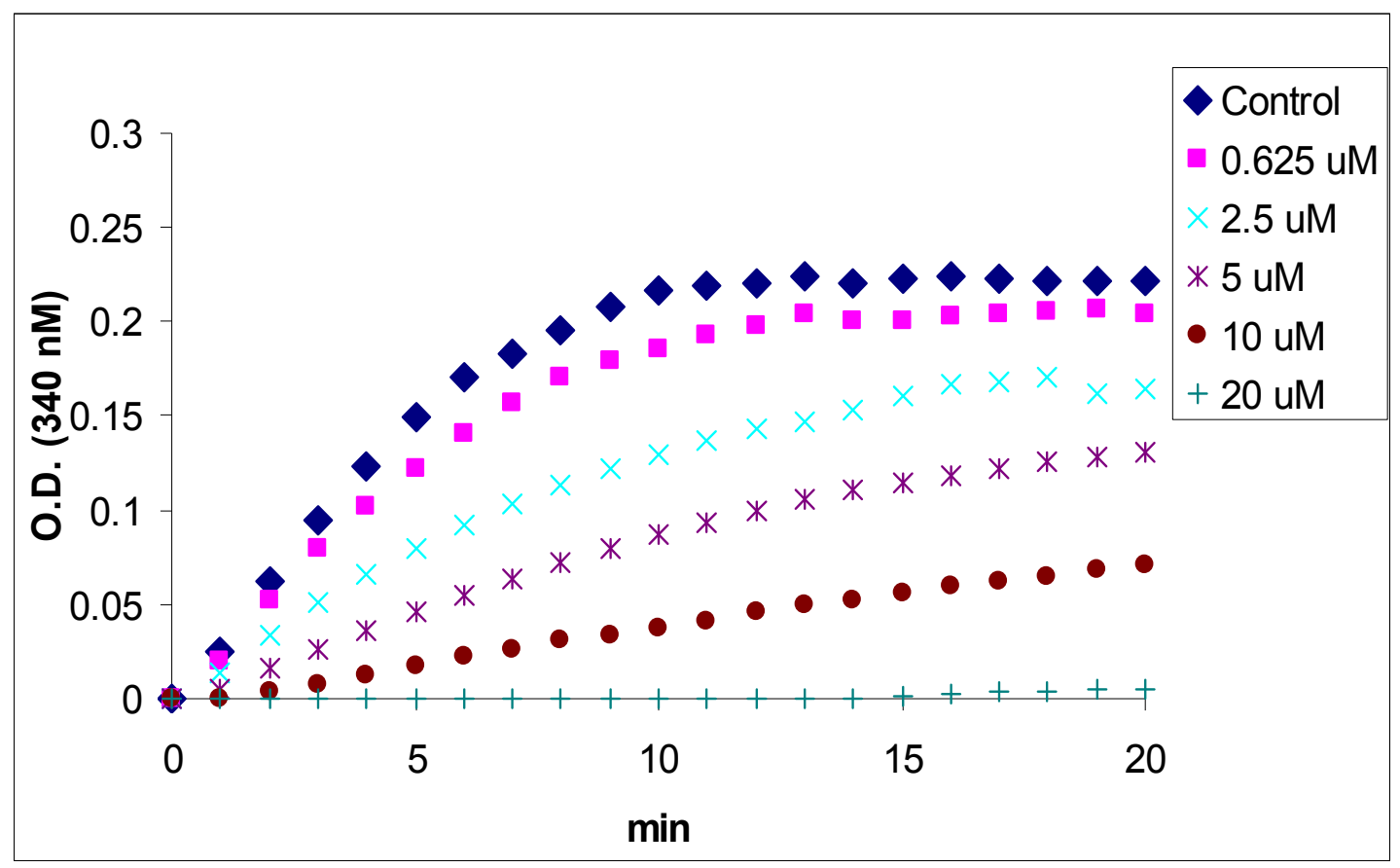

Figure 4-6. SMART-100 inhibited in vitro tubulin polymerization in a dose dependent manner.

This experiment was done by Mr. Chien-Ming Li, Division of Pharmaceutics, College of Pharmacy, the Ohio State University. 
$[3 \mathrm{H}]$ colchicine competition-binding assay was used to confirm that SMART bind to tubulin at the colchicine binding site. In the meantime, I also measured the relative binding affinity. Binding graph (Figure 4-7) showed that: 1), SMART compounds can competitively bind to tubulin colchicine binding site. As concentration of these three testing compounds increased from $0.1 \mu \mathrm{M}$ to $100 \mu \mathrm{M}$, more and more tritiated colchicine were competitively stripped away from tubulin. Thus gave out lower and lower SPA counts. 2), SMART compounds have relatively high binding affinity to the tubulin colchicine binding site. Table 4-7 Showed that software calculated $\mathrm{IC}_{50}$ value for binding. SMART-100 has the highest binding affinity among the three tested compounds. Its value is close to colchicine, although a little bit higher than that. 3 ), the binding affinity was positively correlated with in vitro anti-melanoma activity. The higher of the binding affinity, the higher is the anti-melanoma activity. In our experiment, the D value is 0.08 $\mu \mathrm{M}$, the $\mathrm{Kd}$ value is around $1 \mu \mathrm{M}$ according to literature (131). So the $\mathrm{IC}_{50}$ is very close to $\mathrm{Ki}$ value. Our result is quite comparable with literature reported $\mathrm{Ki}$ value for colchicine binding to tubulin. On the binding graph, some data point is a little bit below 0 . That is caused by experimental variation. Theoretically, the maximum inhibition is 0 .

Microtubule images (Figure 4-8) showed that all four tested compounds results in cytoskeleton rearrangements. There is a significant difference between paclitaxel and other two compounds (Colchicine and SMART-100). Treatment with paclitaxel resulted in condensation of microtubules nice surrounding the nuclei compared with control, consistent with its mechanisms of action for stabilizing microtubules. On the contrary, treatment with colchicine or SMART-100 all has similar effect on microtubules, and result in some degree of microtubule fragmentations, consistent with their common mechanism of action for destabilize microtubules.

In summary, our data clearly showed that SMARTs triggered several molecular events, which include binding to colchicine site of tubulin, inhibiting on tubulin polymerization, arresting cells in G2/M phase and resulting microtubule fragmentation.

\subsubsection{Pharmacokinetic Properties of SMART-100, SMART-45 and SMART-211}

I first examined SMART-100 at a dose level of $5 \mathrm{mg} / \mathrm{kg}$ (Figure 4-9). The plasma concentration-time profiles of SMART-100 after single IV and IP administration are shown in panel A and B of Figure 4-9. The derived pharmacokinetic parameters are shown in Table 4-8. The formulation I used for both injections were DMSO/PEG300 (80/20). SMART-100 appeared rapidly in plasma after IP injection with peak level $\left(\mathrm{C}_{\max }\right)$ observed in less than 2 min after administration. This suggested a rapid absorption of the compound from the injection site. The compound was distributed extensively in the body with a volume of distribution of $112 \mathrm{~L} / \mathrm{kg}$. A very high clearance of $223 \mathrm{~mL} / \mathrm{min} / \mathrm{kg}$ indicates extensive metabolism and rapid elimination. The high clearance resulted in a relative short terminal half-life of 349 min after IV and 358 min after IP administration despite of the huge volume of distribution. The IP bioavailability is only $31 \%$. I thought the poor bioavailability was largely due to the poor water solubility of the compound and too simple formulation. 


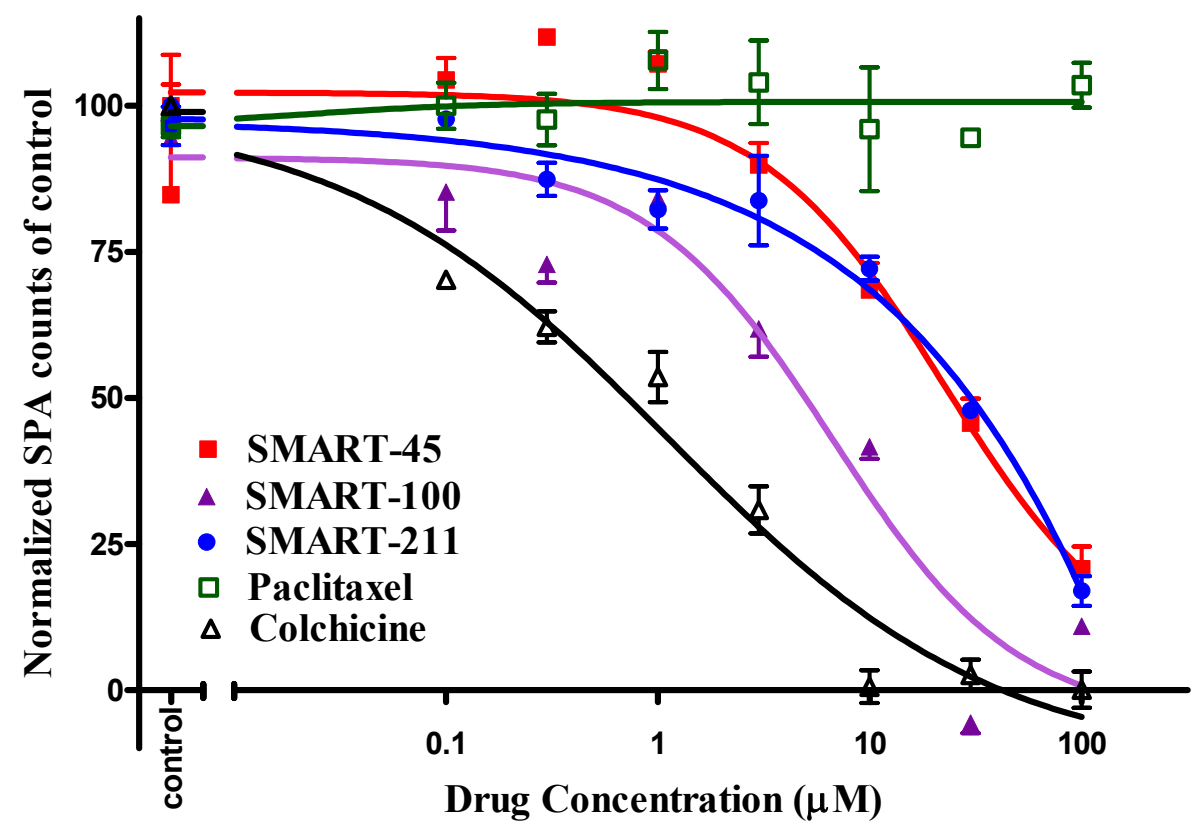

Figure 4-7. Tubulin binding assay showed SMART compounds competitively bound to tubulin colchicine binding site in a concentration dependant manner.

As concentration of these compounds increased from $0.1 \mu \mathrm{M}$ to $100 \mu \mathrm{M}$, more and more tritiated colchicine were competitively stripped away from tubulin. Thus gave out lower and lower SPA counts. The negative control paclitaxel just gave out a flat line because it didn't bind to the colchicine binding site on tubulin. Each data point represents an average of three replicates.

Table 4-7. Comparison of tubulin binding affinity and in vitro cytotoxic activity between SMART compounds and known tubulin interacting drugs.

\begin{tabular}{ccc}
\hline \multirow{2}{*}{ Compounds } & \multicolumn{2}{c}{$\mathrm{IC}_{50} \pm \mathrm{SEM}(\mathrm{n}=3)$} \\
\cline { 2 - 3 } & For tubulin binding $(\mu \mathrm{M})$ & On $\mathrm{A} 375(\mathrm{nM})$ \\
\hline SMART-100 & $7 \pm 3$ & $28 \pm 5$ \\
SMART-45 & $22 \pm 4$ & $29 \pm 11$ \\
SMART-211 & $38 \pm 2$ & $93 \pm 11$ \\
Colchicine & $1 \pm 0$ & $20 \pm 3$ \\
Paclitaxel & N/A & $12 \pm 3$ \\
\hline
\end{tabular}




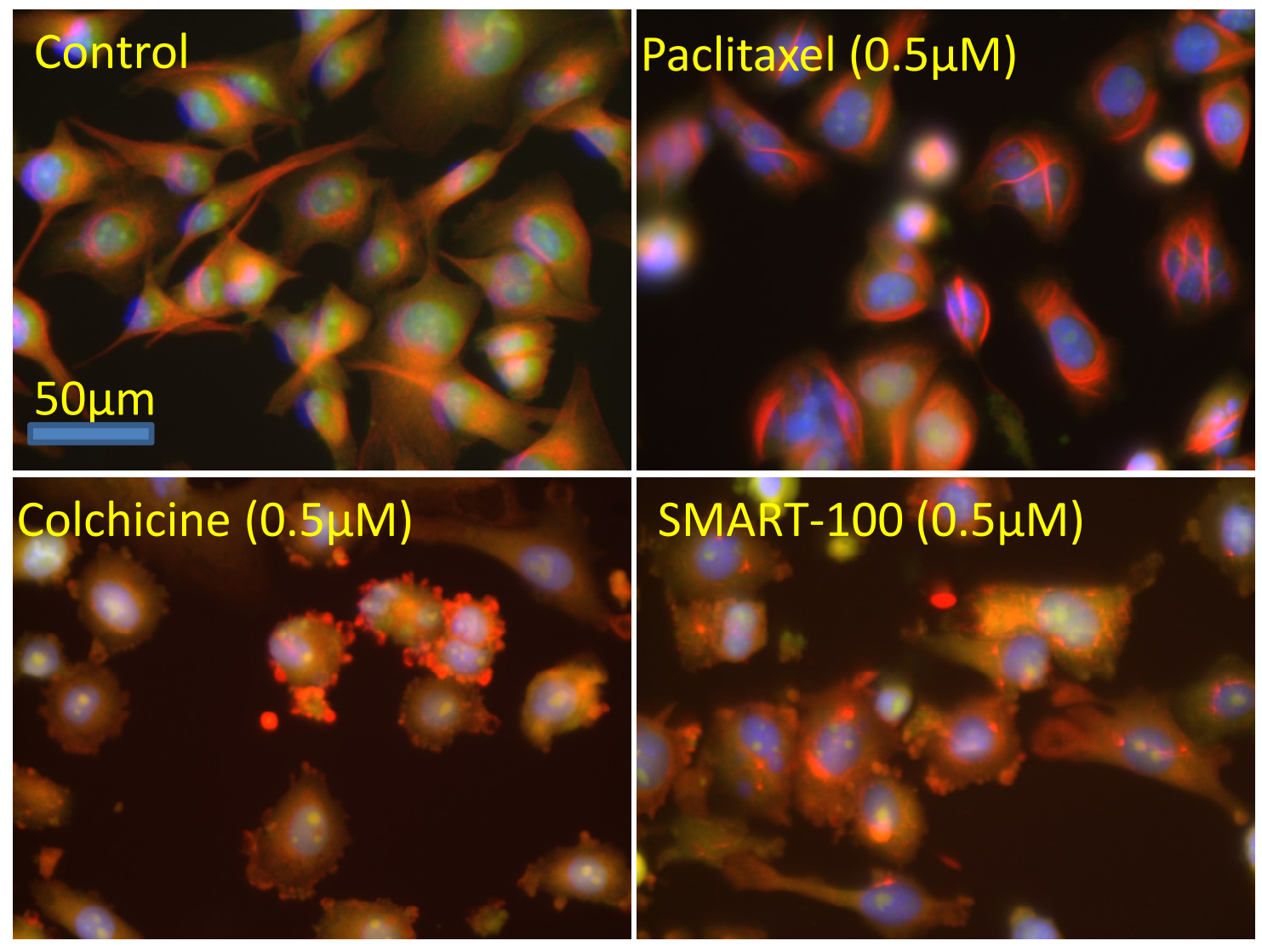

Figure 4-8. Microscopic images of immunofluorescence labeled microtubules inside WM-164 melanoma cells showed microtubule modality was dramatically changed after compounds treatment for $18 \mathrm{~h}$. 
A

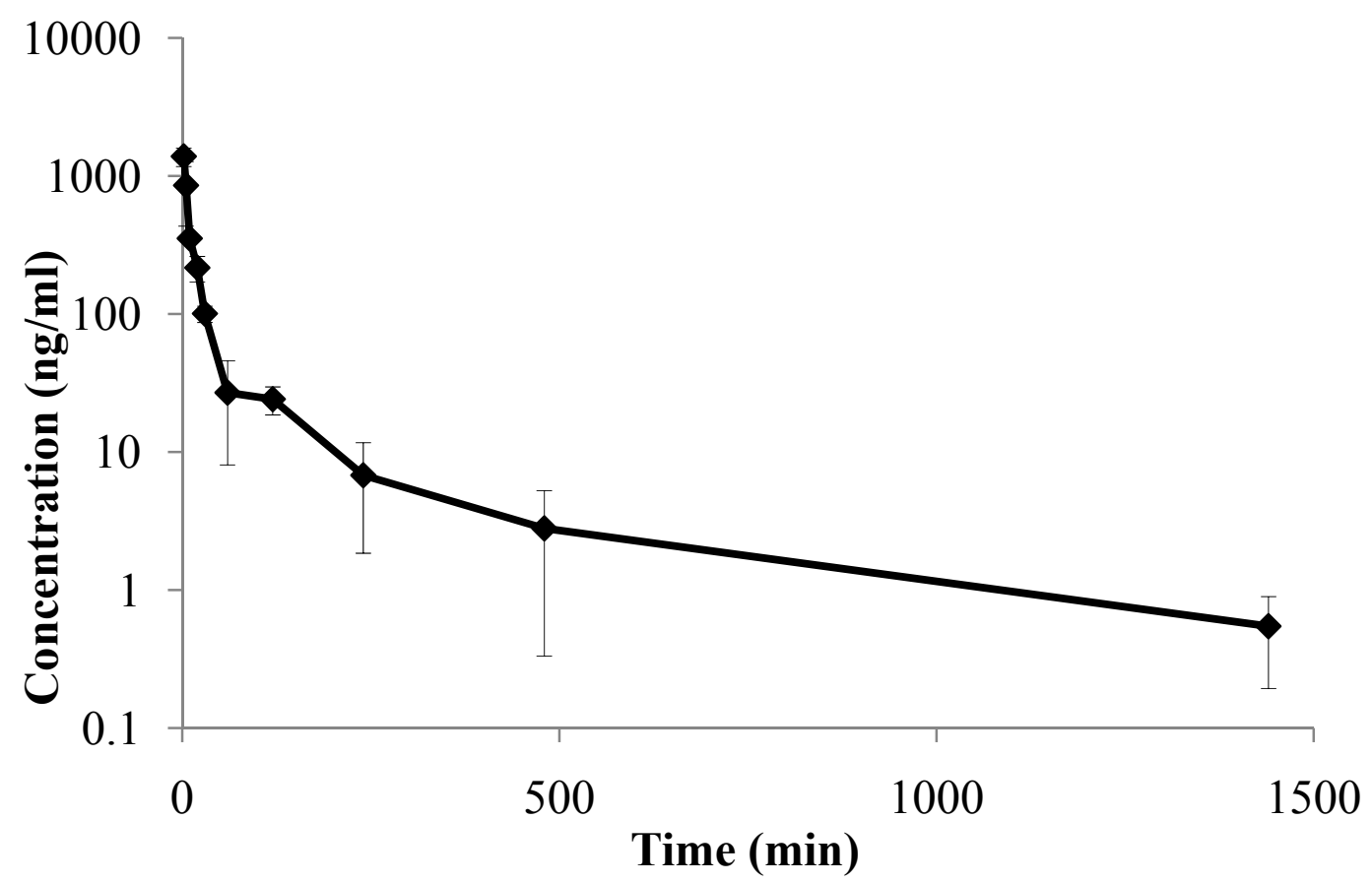

B

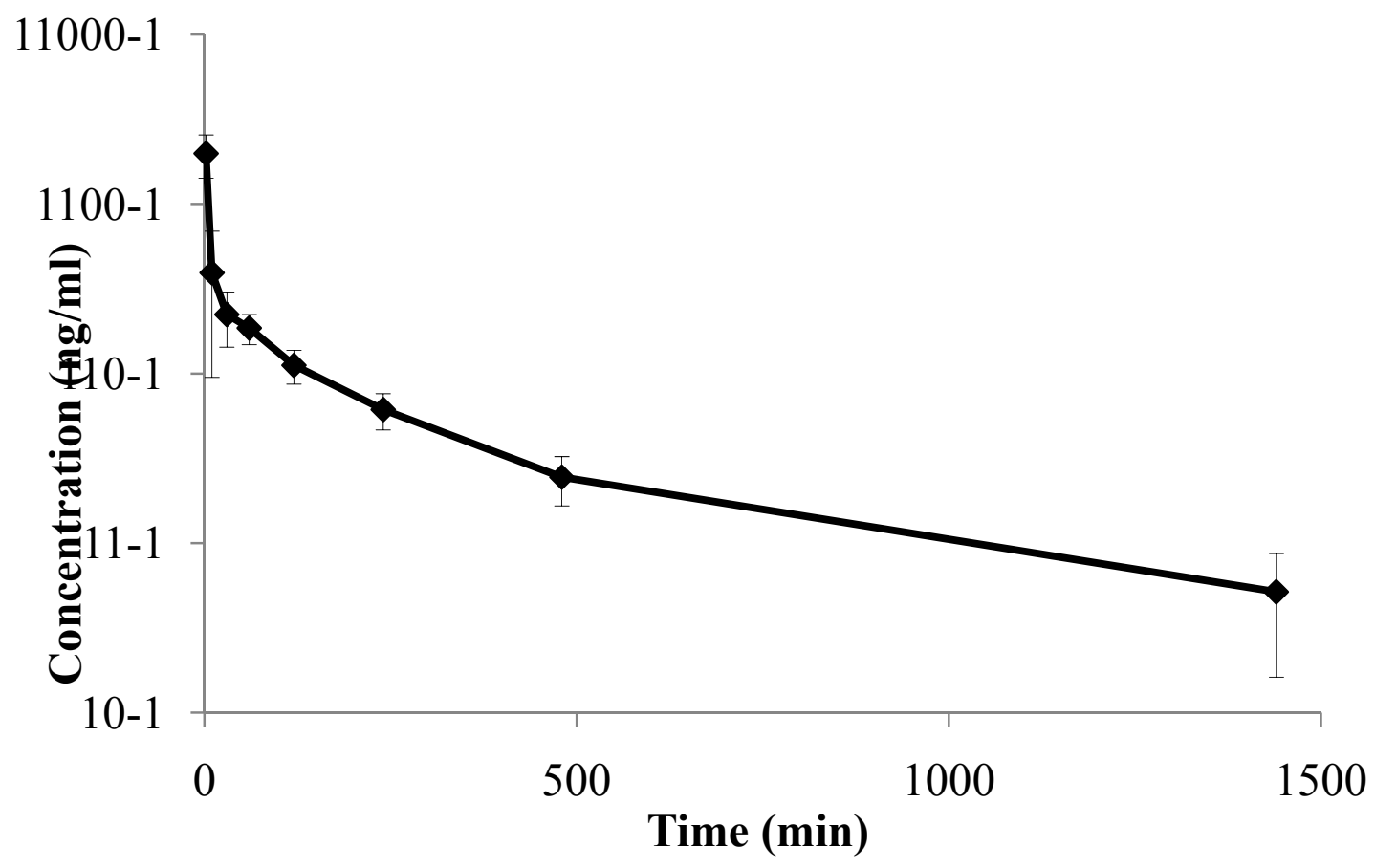

Figure 4-9. Measured plasma concentration-time profiles (mean $\pm \mathrm{SD}$ ) after bolus administration of $5 \mathrm{mg} / \mathrm{kg}$ SMART-100 to ICR mice ( $\mathrm{n}=3$ per group).

A: i.v. injection, formulation, DMSO:PEG300=80:20 (v/v). B: i.p. injection, formulation, DMSO:PEG300=80:20 (v/v). 
Table 4-8. Pharmacokinetic parameters of SMART-100, SMART-45, and SMART-211.

\begin{tabular}{|c|c|c|c|c|c|c|c|c|}
\hline Route & Compound and dose & $\begin{array}{c}\mathrm{t}_{1 / 2} \\
(\mathrm{~min})\end{array}$ & $\begin{array}{c}\mathrm{AUC}_{\text {inf }} \\
(\mathrm{ng} \cdot \mathrm{min} / \mathrm{mL})\end{array}$ & $\begin{array}{c}\mathrm{AUC}_{\text {last }} \\
(\mathrm{ng} \cdot \mathrm{min} / \mathrm{mL})\end{array}$ & $\begin{array}{c}\text { Clearance } \\
(\mathrm{mL} / \mathrm{min} / \mathrm{kg})\end{array}$ & $\begin{array}{c}\mathrm{V}_{\mathrm{z}} \\
(\mathrm{mL} / \mathrm{kg})\end{array}$ & $\begin{array}{c}\mathrm{V}_{\mathrm{ss}} \\
(\mathrm{mL} / \mathrm{kg})\end{array}$ & $\begin{array}{c}\text { Bioavailability } \\
(\%)\end{array}$ \\
\hline \multirow{4}{*}{ IV } & SMART-100 $5 \mathrm{mg} / \mathrm{kg}^{\mathrm{a}}$ & 349 & 22434 & 22159 & 223 & 112311 & 24803 & \\
\hline & SMART-100 $15 \mathrm{mg} / \mathrm{kg}^{\mathrm{a}}$ & 140 & 115336 & 115221 & 130 & 26274 & 4926 & \\
\hline & SMART-45 $15 \mathrm{mg} / \mathrm{kg}^{\mathrm{a}}$ & 141 & 134340 & 134051 & 112 & 22643 & 6588 & \\
\hline & SMART-211 $15 \mathrm{mg} / \mathrm{kg}^{\mathrm{a}}$ & 334 & 168759 & 151526 & 95 & 45954 & 5420 & \\
\hline \multirow{5}{*}{ IP } & SMART-100 $5 \mathrm{mg} / \mathrm{kg}^{\mathrm{a}}$ & 358 & 7038 & 6771 & 710 & 366859 & & 31 \\
\hline & SMART-100 $15 \mathrm{mg} / \mathrm{kg}^{\mathrm{a}}$ & 308 & 17645 & 17189 & 850 & 378339 & & 15 \\
\hline & SMART-100 $15 \mathrm{mg} / \mathrm{kg}^{\mathrm{b}}$ & 123 & 55842 & 55760 & 269 & 47507 & & 48 \\
\hline & SMART-45 $15 \mathrm{mg} / \mathrm{kg}^{\mathrm{b}}$ & 182 & 72001 & 71719 & 208 & 54674 & & 54 \\
\hline & SMART-211 $15 \mathrm{mg} / \mathrm{kg}^{\mathrm{b}}$ & 208 & 122578 & 122440 & 122 & 36700 & & 73 \\
\hline
\end{tabular}

${ }^{\mathrm{a} C}$ Compound was formulated with DMSO:PEG300=80:20 (v/v).

${ }^{\mathrm{b}}$ Compound was formulated with Tween80:Captex200=80:20 (v/v). 
I then examined SMART-100 at a dose level of $15 \mathrm{mg} / \mathrm{kg}$ and compared two different formulations for IP injection. One is DMSO/PEG300 (80/20), one is

Tween80/captex200 (80/20). The later formulation was one of the self-emulsifying drug delivery systems (SEDDS), which contained a mixture of oil (captex200) and surfactant (Tween80), and improved the solubility of our lipophilic SMARTs (132). The plasma concentration-time profiles of SMART-100 after single IV and IP administration are shown in panel A, B, and C of Figure 4-10. The derived pharmacokinetic parameters are shown in Table 4-8. Compared different dose of SMART-100 with the same DMSO/PEG300 (80/20) formulation, higher dose (15 mg/kg) gave lower bioavailability (15\% vs. 31\%). This may be due to higher concentrated SMART-100 in DMSO/PEG300 $(80 / 20)$ precipitated out more after IP injection. Thus more compounds became unavailable for absorption. Absorption of SMART-100 after IP injection with Tween80/captex200 (80/20) formulation was slower than DMSO/PEG300 (80/20). The former one had a $t_{\max }$ of $15 \mathrm{~min} v s$. the later one of $1 \mathrm{~min}$. That's because the former formulation delayed the release of compound. With the help of this new formulation, the bioavailability was increased from $15 \%$ to $48 \% .15 \mathrm{mg} / \mathrm{kg}$ of SMART-100 had 5.1-fold area on under the curve (AUC) than the dose with $5 \mathrm{mg} / \mathrm{kg}$. The other parameters like $\mathrm{t}_{1 / 2}$, volume of distribution, and clearance also changed dramatically along with dose change. This suggested that SMART-100 may have non-linear kinetics.

I next examined SMART-45 and SMART-211 at a dose level of $15 \mathrm{mg} / \mathrm{kg}$ with the new formulation for IP injection. The plasma concentration-time profiles of SMART100 after single IV and IP administration are shown in panel A and B of Figure 4-11 and Figure 4-12. The derived pharmacokinetic parameters are shown in Table 4-8. The IP bioavailability of SMART-45 and-SMART-211 were 54\% and 73\% respectively. This confirmed the new formulation's ability to increase bioavailability. I then used this formulation for our further in vivo xenograft studies, and no toxic effects were found in the vehicle group of A375 xenograft models, suggesting that this formulation was safe to use for long-term (more than one month) xenograft studies.

With the same dose of $15 \mathrm{mg} / \mathrm{kg}$ of SMART-45 and SMART-100, the parameters of AUC (min $\bullet \mathrm{ng} / \mathrm{mL}), \mathrm{t}_{1 / 2}(\mathrm{~min}), \mathrm{V}_{\mathrm{z}}(\mathrm{L} / \mathrm{kg})$, and $\mathrm{CL}(\mathrm{mL} / \mathrm{min} / \mathrm{kg})$ were very close to each other. That can be understood by their similarity of chemical structure and lipiphilicty. SMART-211 has relative lower clearance and thus its terminal half life was relatively longer. But overall SMART compounds exhibited high clearances and short half-life. Once-daily regimen was designed to improve compounds exposure and treatment efficacy for our xenograft studies.

The calculated clearances of SMART compounds are much higher than the mouse hepatic blood flow rate. There maybe three reasons account for this. First, this may be due to expetimental error. Especially when drug concentration is close to detection limit, the measurement error is relatively large. Second, this may be due to incomplete tail vein injection. The drug amount actually got into the vein may be is less then what we planned. Third, SMART compounds may have extra-hepatic metabolism. For example, the enzyme in the blood, speen, heart or other organs may be can metablize these compounds. 
A

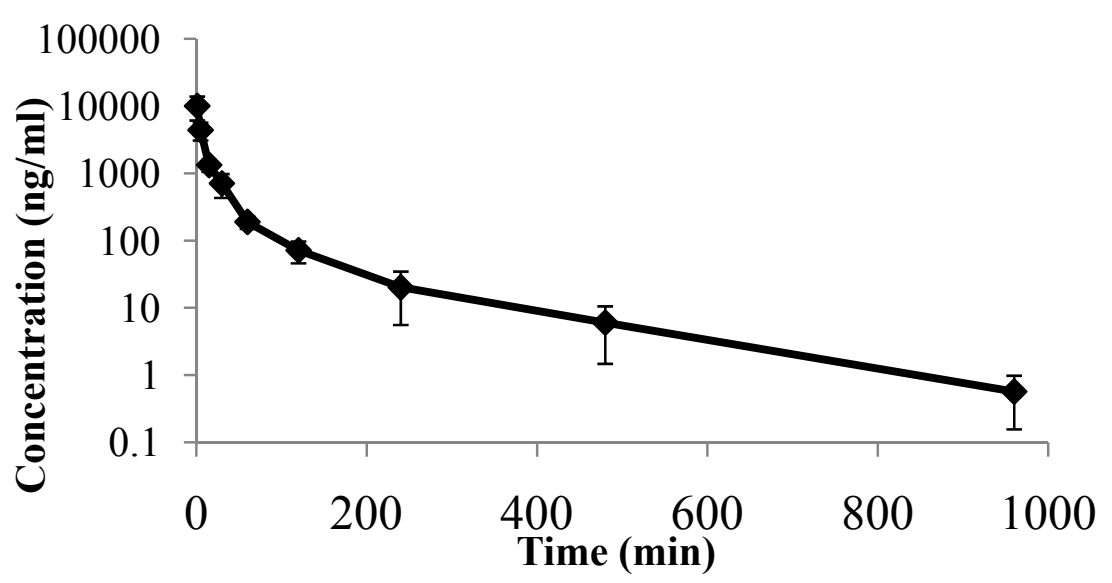

B

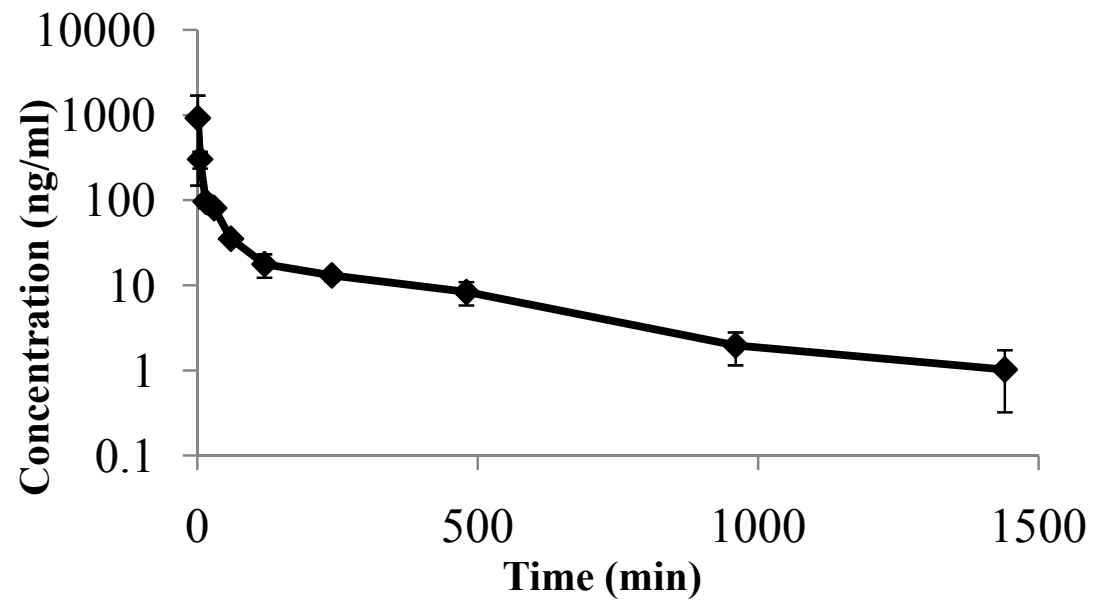

C

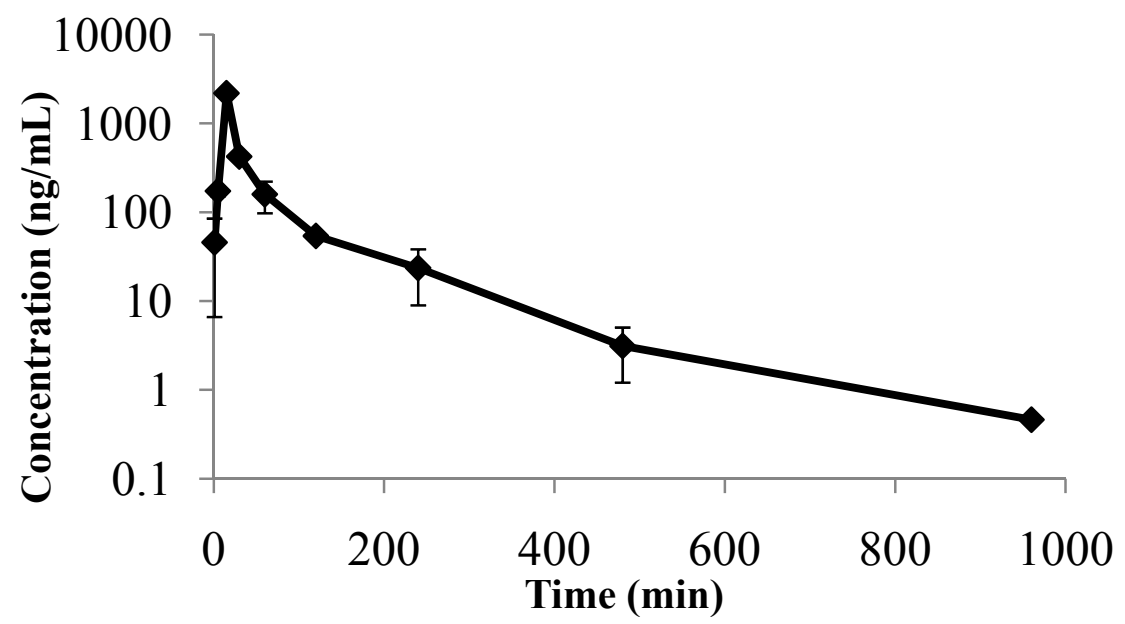

Figure 4-10. Measured plasma concentration-time profiles (mean $\pm \mathrm{SD}$ ) after bolus administration of $15 \mathrm{mg} / \mathrm{kg}$ SMART-100 to ICR mice ( $\mathrm{n}=3$ per group). A: i.v. injection, formulation, DMSO:PEG300=80:20 (v/v). B: i.p. injection, formulation, DMSO:PEG300=80:20 (v/v). C: i.p. injection, formulation, Tween80:Captex200=80:20 (v/v). 
A

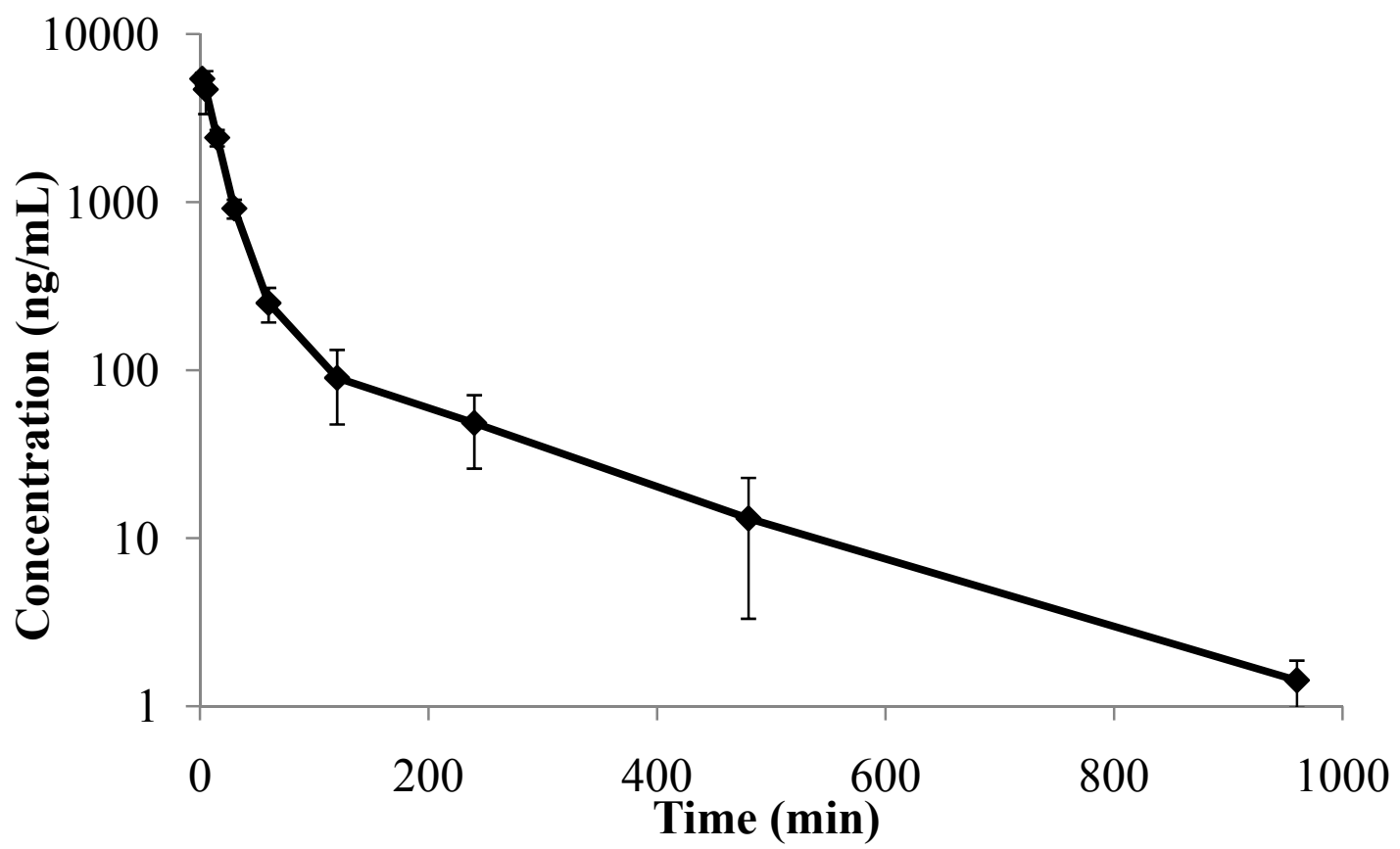

B

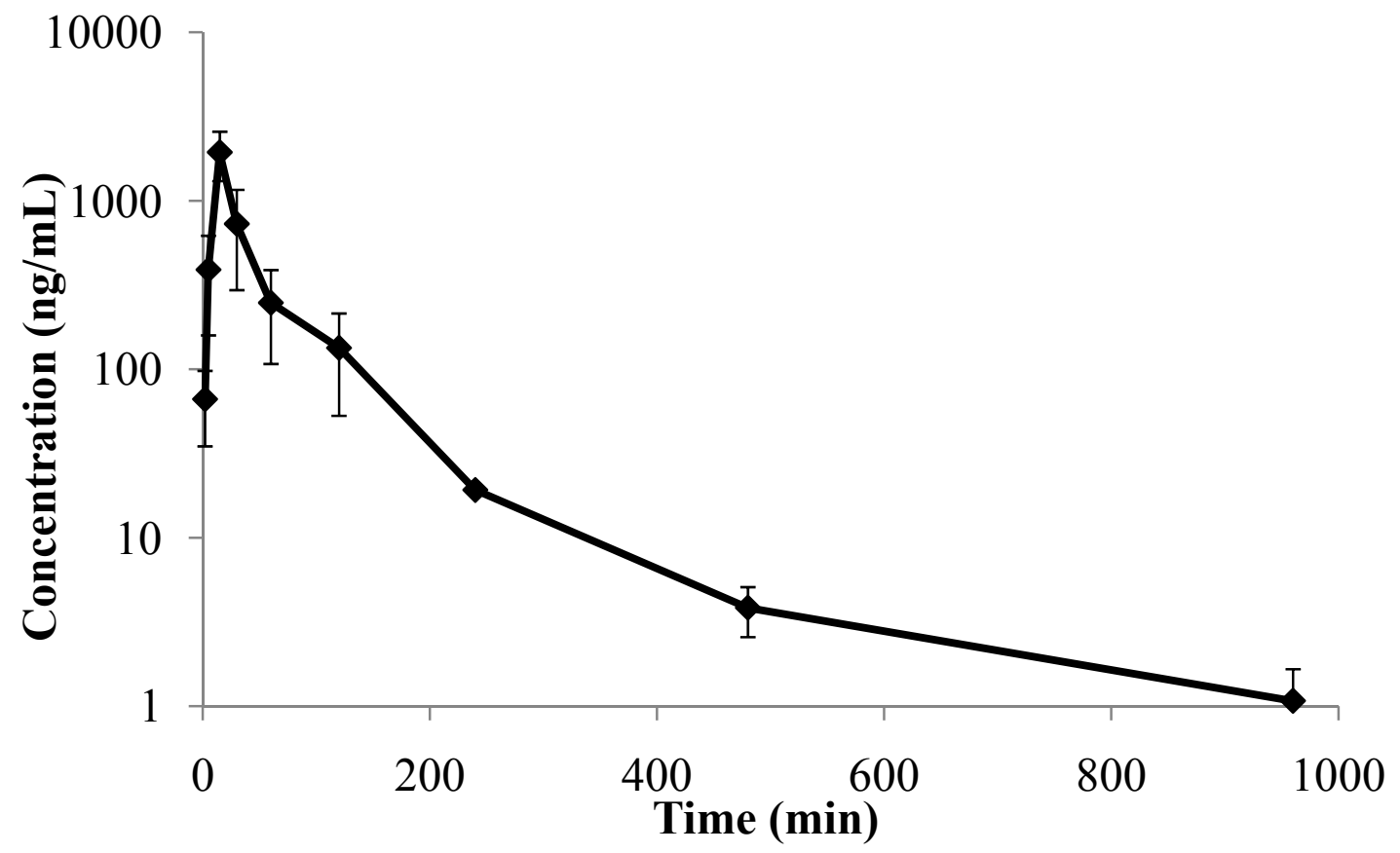

Figure 4-11. Measured plasma concentration-time profiles (mean $\pm \mathrm{SD}$ ) after bolus administration of $15 \mathrm{mg} / \mathrm{kg}$ SMART- 45 to ICR mice ( $\mathrm{n}=3$ per group).

A: i.v. injection, formulation, DMSO:PEG300=80:20 (v/v). B: i.p. injection, formulation, Tween80:Captex200=80:20 (v/v). 
A

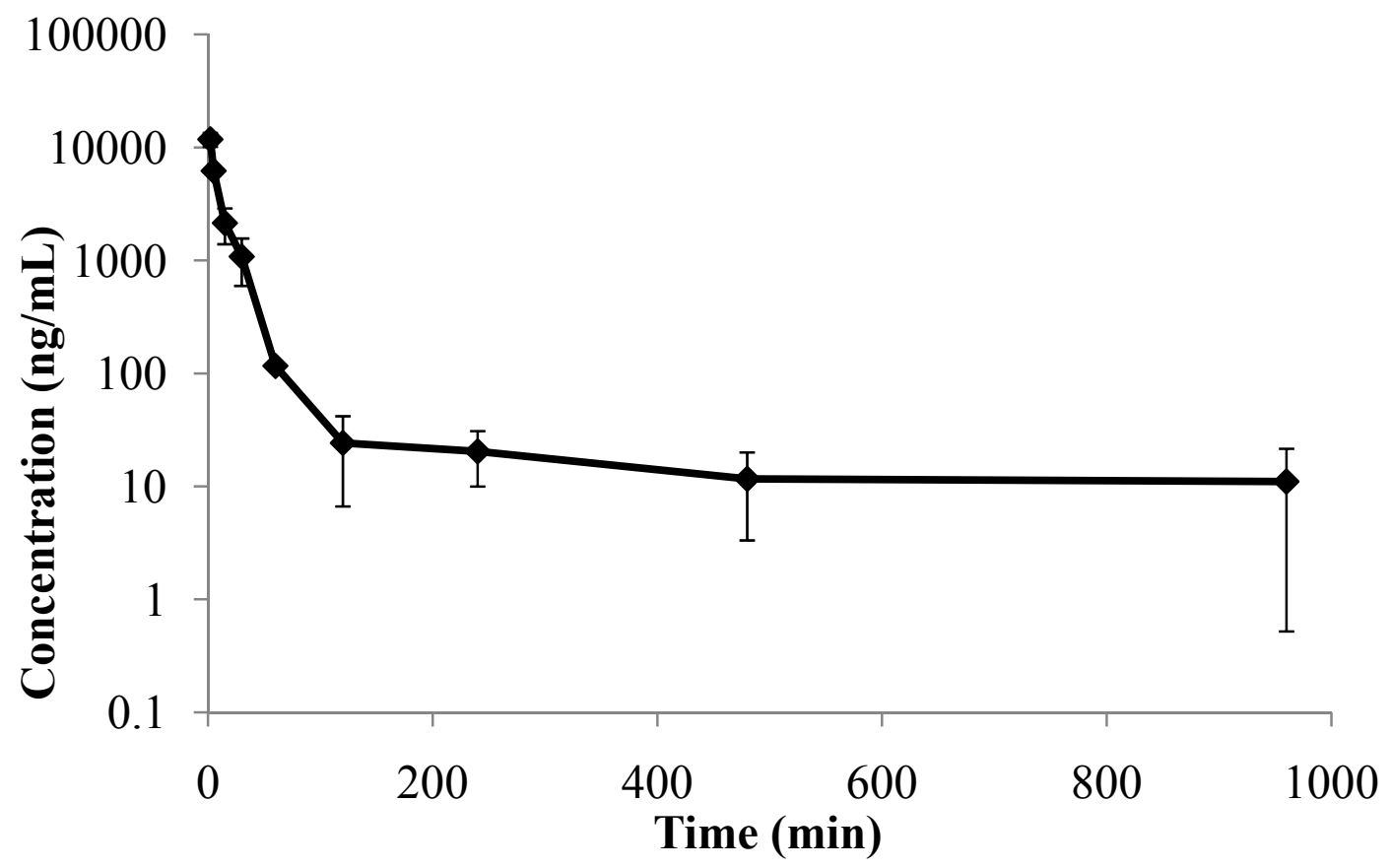

B

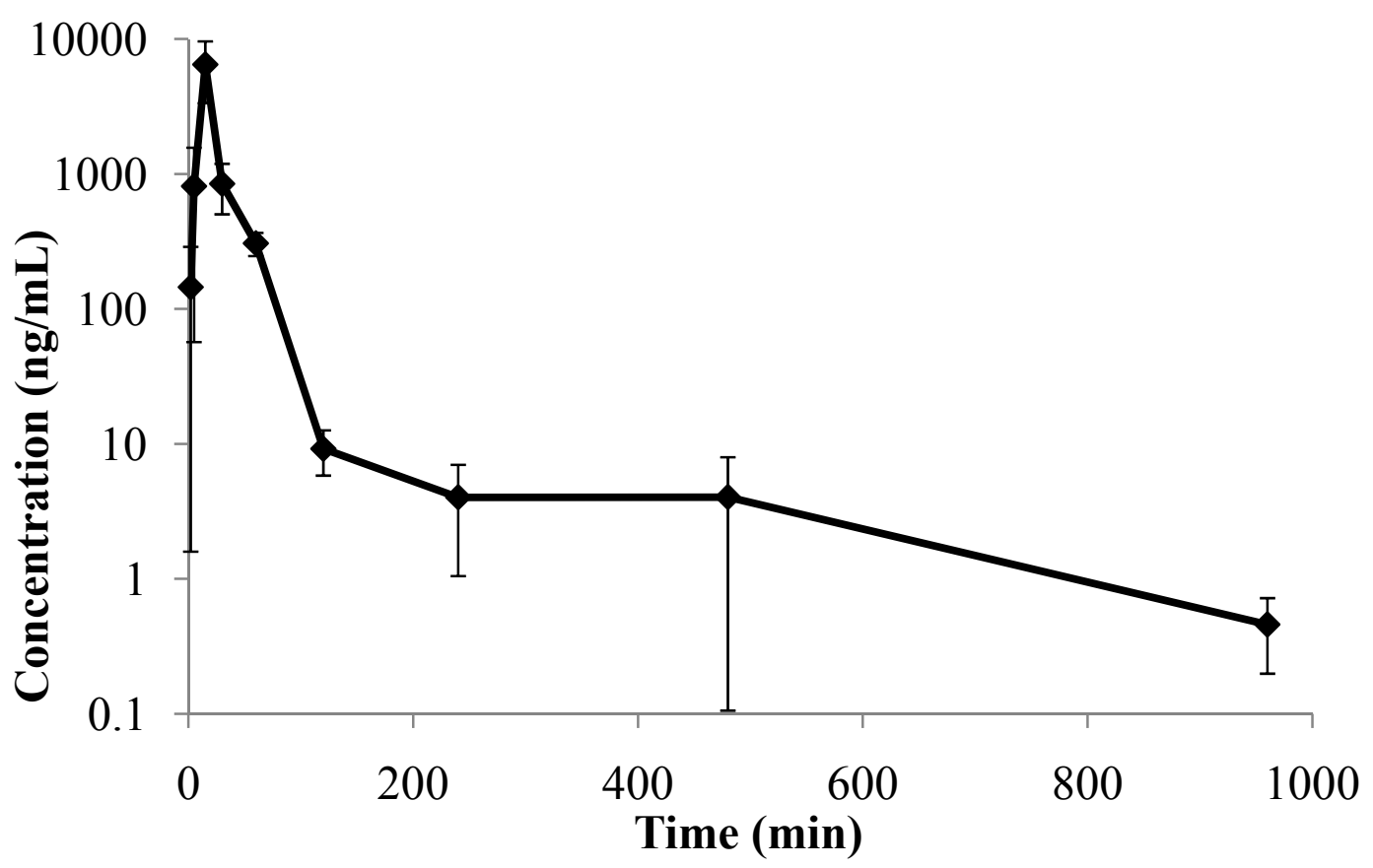

Figure 4-12. Measured plasma concentration-time profiles (mean $\pm \mathrm{SD}$ ) after bolus administration of $15 \mathrm{mg} / \mathrm{kg}$ SMART-211 to ICR mice ( $\mathrm{n}=3$ per group). A: i.v. injection, formulation, DMSO:PEG300=80:20 (v/v). B: i.p. injection, formulation, Tween80:Captex200=80:20 (v/v). 


\subsubsection{Antitumor Efficacy of SMARTs on A375 Xenograft Model}

I examined $20 \mathrm{mg} / \mathrm{kg}$ of SMART-100 and $15 \mathrm{mg} / \mathrm{kg}$ of SMART-45 treatments on A375 human melanoma xenograft model. Nude mice were administrated i.p. every day for consecutive 32 days when the tumor size was $\sim 150 \mathrm{~mm}^{3}$. As shown in Figure 4-13, SMART-100 and SMART-45 exhibited significant inhibition of tumor growth. Summary of the data was presented in Table 4-9. The average tumor size in control group increased by about 13 folds. I obtained 4.8-fold and 4.2-fold tumor increases in SMART-100 treated $(20 \mathrm{mg} / \mathrm{kg})$ and SMART-45 treated $(15 \mathrm{mg} / \mathrm{kg})$ groups, respectively. SMART100 reduced tumor growth rate with a $\%$ TGI of $70 \%(20 \mathrm{mg} / \mathrm{kg}$ treatment $)$, and SMART45 inhibited $72 \%$ (15 mg/kg treatment). I also calculated $\% \mathrm{~T} / \mathrm{C}$ for our efficacy study. With $20 \mathrm{mg} / \mathrm{kg}$ treatment of SMART-100 and $15 \mathrm{mg} / \mathrm{kg}$ treatment of SMART-45 on A375 xenograft, I observed \% $/ \mathrm{C}$ values of $36 \%$ and $33 \%$, respectively. The data indicated that SMART-100 and -45 were pretty active in inhibiting A375 xenografts growth on athymic nude mice.

In the mean time, the general activities of mice in all groups were normal during the treatment period. At the conclusion of the experiment, the group average body weight increased by 3.1g for control group, 2.2g for SMART-45 treated group, and $2.4 \mathrm{~g}$ for SMART-100 treated group. Corrected by group average tumor weight, which were $1.3 \mathrm{~g}$ for control group, 0.6g for SMART-45 treated group, and 0.6g for SMART-100 treated group, the net group body weight gain were $1.8 \mathrm{~g}$ for control group, $1.6 \mathrm{~g}$ for SMART-45 treated group, and 1.8g for SMART-100 treated group. One mouse in control group, one mouse in SMART-100 treated group, and two mice in SMART-45 treated group died during the experiment period. I also observed big hard lumps in the injection sites of dead mice. So I guess the death of the mice was due to injection, not the toxicity of compounds treatment. No any other acute toxicity phenomenon was observed.

\subsection{Conclusions}

We have discovered a new class of simple synthetic compounds based on a 2aryl-4-(3, 4, 5-trimethoxylbenzoyl)-thiazole molecular skeleton, which was derived from thiazolidine ring modification of ATCAA structures. Chemical modification of different substituted aryl in A and $\mathrm{C}$ rings and structure-activity relationship of the SMART were investigated based on biological evaluation against melanoma cells in vitro. I have demonstrated that SMARTs are very potent in inhibiting melanoma cell proliferation in vitro. In the meantime, our data also showed SMARTs overcame multi-drug resistance in drug resistant melanoma cell line, suggesting they are not substrates for P-gp. Mechanism of action studies showed these compounds bound tubulin at the colchicine binding site thus inhibit tubulin polymerization. The in vivo efficacy studies showed that SMARTs significantly inhibited A375 melanoma tumor growth on nude mice xenograft models. The dramatic biologic effect of SMARTs suggested that it has great potential for melanoma chemotherapy. 


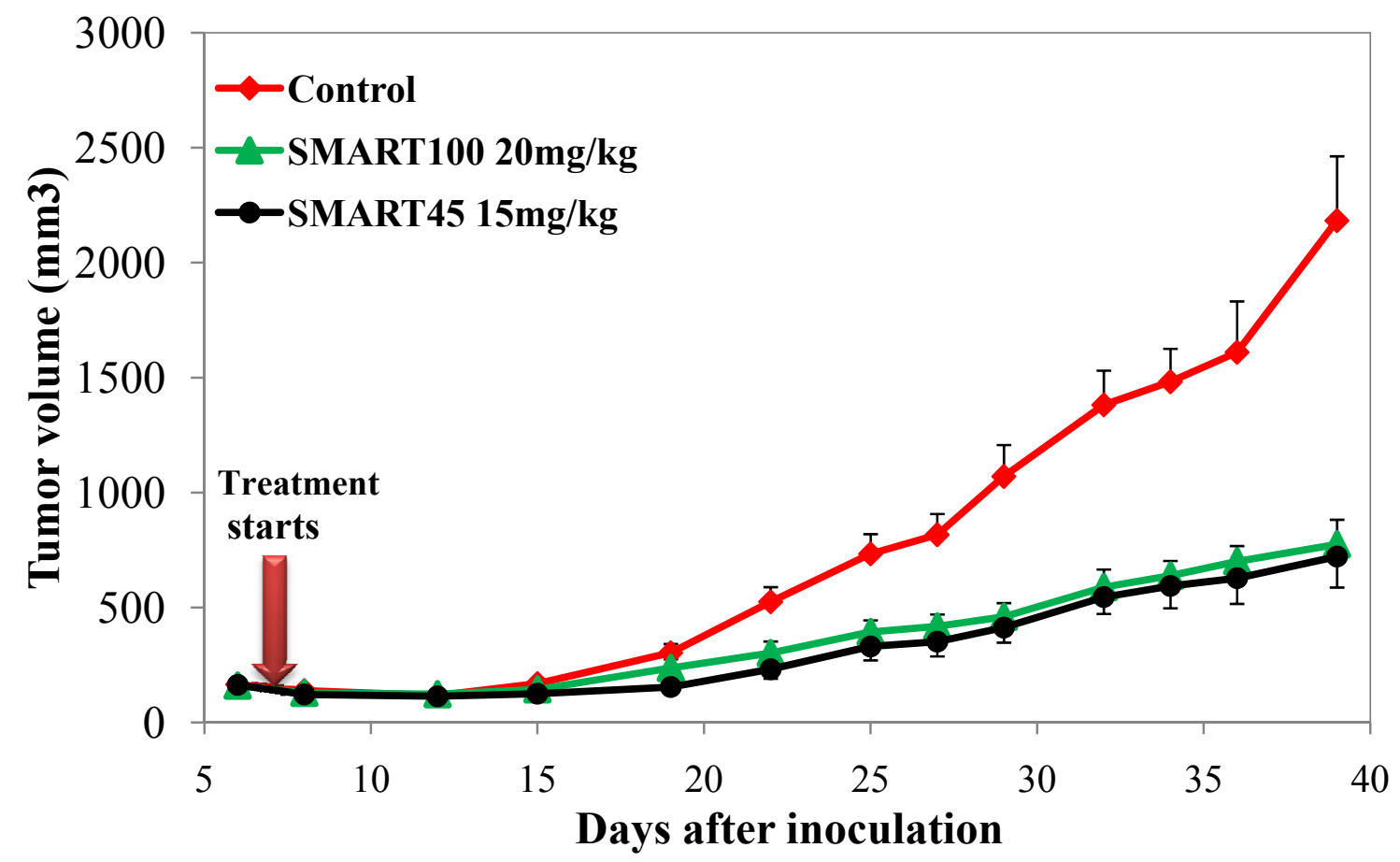

Figure 4-13. In vivo efficacy of SMARTs on nude mice bearing A375 melanoma xenograft tumor ( $\mathrm{n}=10$ per group).

A375 cells $\left(2.5 \times 10^{6}\right)$ were injected s.c. on the right flank. The compounds were given by i.p. daily when tumor size was $150 \mathrm{~mm}^{3}$. 
Table 4-9. Summary of mice number, mice body weight change, and tumor size change during in vivo efficacy experiment.

\begin{tabular}{|c|c|c|c|c|c|c|c|c|}
\hline \multirow{2}{*}{ Groups } & \multicolumn{2}{|c|}{$\begin{array}{c}\text { Number of } \\
\text { animals }\end{array}$} & \multicolumn{2}{|c|}{$\begin{array}{c}\text { Body weight (g) } \\
\text { Mean } \pm \text { SEM }\end{array}$} & \multicolumn{2}{|c|}{$\begin{array}{c}\text { Tumor size }\left(\mathrm{mm}^{3}\right) \\
\text { Mean } \pm \text { SEM }\end{array}$} & \multirow[t]{2}{*}{$\mathrm{TGI}^{\mathrm{a}}$} & \multirow[t]{2}{*}{$T / C^{b}$} \\
\hline & Start & End & Start & End & Start & End & & \\
\hline $\begin{array}{l}\text { Vehicle } \\
\text { control }\end{array}$ & 10 & 9 & $19 \pm 2$ & $22 \pm 3$ & $165 \pm 11$ & $2183 \pm 279$ & N/A & N/A \\
\hline $\begin{array}{l}\text { SMART-100, } \\
20 \mathrm{mg} / \mathrm{kg}\end{array}$ & 10 & 9 & $19 \pm 2$ & $21 \pm 2$ & $160 \pm 15$ & $775 \pm 107$ & $70 \%$ & $36 \%$ \\
\hline $\begin{array}{c}\text { SMART-45, } \\
15 \mathrm{mg} / \mathrm{kg}\end{array}$ & 10 & 8 & $19 \pm 2$ & $21 \pm 3$ & $164 \pm 12$ & $722 \pm 135$ & $72 \%$ & $33 \%$ \\
\hline
\end{tabular}

${ }^{a}$ TGI (tumor growth inhibition) were measured on the final day for drug-treated compared with vehicle-treated mice and calculated as $100 \times\{1-[($ Treated, final Treated,day1)/(Control, final - Control, day1)]\}.

${ }^{\mathrm{b}} \mathrm{T} / \mathrm{C}$ were generated from the mean-treated tumor volume on the final day divided by the mean control tumor volume on the final day times 100 . 


\section{CHAPTER 5. NOVEL IMIDAZOLES TARGET TUBULIN POLYMERIZATION AND OVERCOME MULTI-DRUG RESISTANCE IN METASTATIC MELANOMA}

\subsection{Introduction}

I have previously dicussed the discovery of substituted methoxylbenzoyl-arylthiazole (SMART) compounds that showed good anti-melanoma activity both in vitro and in vivo (100). But poor solubility and fast metabolism were two major problems associated with these compounds. After a thorough structure-activity relationship (SAR) study and extensive lead compound modification, we discovered a new class of compounds: 2-aryl-4-benzoyl-imidazoles (ABIs). The general structure of this class of compounds is shown in Figure 5-1. Mr. Jianjun Chen used an imidazole ring to replace a thiazole ring for the following two reasons: (a) To increase water solubility. The imidazole ring has a lower LogP value and a higher $\mathrm{pKa}$ value compared with the thiazole ring. Thus, imidazole compounds are more hydrophilic and more basic. It is also easier to make them into salt, thereby further increasing water solubility. (b) To increase metabolic stability. The thiazole ring is liable to oxidation, so it can make the whole molecule unstable to cytochrome enzymes (133).

To our great satisfaction, I have identified experimentally two advantages of ABIs over SMART compounds: (a) slightly increased in vitro activity. The $\mathrm{IC}_{50}$ s of some ABIs were in the low nanomolar range on several melanoma cell lines. (b) I have discovered that the trimethoxyphenyl substituent can be replaced by a metabolically more stable pfluorophenyl ring and retain good anticancer activity. In this chapter, I will summarize more findinga about ABIs such as their effect on MDR melanoma cells, effect on melanoma colony formation, and in vivo anti-melanoma efficacy. I also will explain how we identify the cellular target of ABIs.

\subsection{Materials and Methods}

\subsubsection{Compounds and Reagents}

Compound ABI-270 \{IUPAC name: (4-Fluoro-phenyl)-[2-(4-methoxy-phenyl)1H-imidazol-4-yl]-methanone , compound ABI-274 [IUPAC name: (2-p-Tolyl-1Himidazol-4-yl)-(3,4,5-trimethoxy-phenyl)-methanone], and compound ABI-288 \{IUPAC name: [2-(4-Chloro-phenyl)-1H-imidazol-4-yl]-(4-fluoro-phenyl)-methanone 3 were synthesized by Mr. Jianjun Chen in our lab. Bortezomib was obtained from Millennium Pharmaceuticals, Inc. (Kansas City, MO) through a material transfer agreement. Compound ABT-751 was synthesized by Dr. Yan Lu. Paclitaxel, vinblastine, colchicine, dimethyl sulphoxide (DMSO), and Tween 80 were obtained from Sigma-Aldrich (St. Louis, MO). Captex 200 was obtained from ABITEC Corporation (Paris, IL). CellTiter 96® AQueous One Solution Reagent was obtained from Promega Corporation (Madison, 


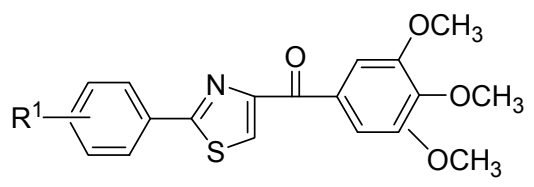

Substituted methoxylbenzoyl-aryl-thiazole (SMART)

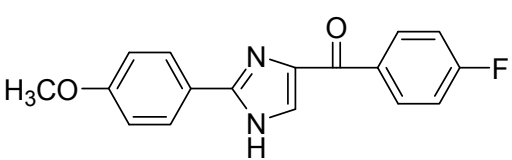

$\mathrm{ABI}-\mathbf{2 7 0}$

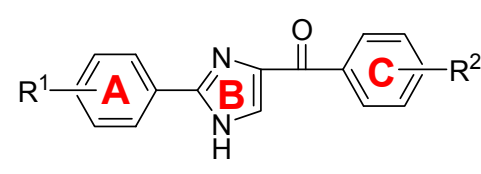

2-aryl-4-benzoyl-imidazoles (ABI)<smiles>COc1cc(C(=O)c2c[nH]c(-c3ccc(C)cc3)n2)cc(OC)c1OC</smiles>

$\mathrm{ABI}-274$

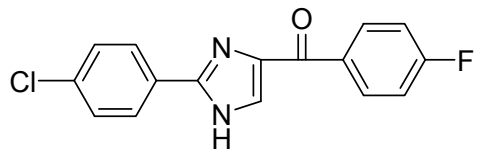

$\mathrm{ABI}-\mathbf{2 8 8}$

Figure 5-1. Structures of SMART, ABI, and three representative ABI compounds: ABI270,274 , and 288. 
WI). DTIC for injection, USP (a formulation for human use), was obtained from APP Pharmaceuticals, LLC (Schaumburg, IL).

\subsubsection{Cell Culture}

The A375 and B16-F1 melanoma cancer cells were obtained from American Type Culture Collection. WM-164 cells were derived from patient metastatic melanoma tumors and were a gift from Dr. Meenhar Herlyn (Wistar Institute, Philadelphia, PA). The P-gp overexpressing multi-drug resistant cell line MDA-MB-435/LCC6MDRI and matching sensitive parent cell line were kindly provided by Dr. Robert Clarke (Georgetown University, Washington, DC). Cells were grown as monolayers by using standard cell culture conditions. All cells were cultured in DMEM (Mediatech, Inc., Manassas, VA), supplemented with 10\% FBS (Atlanta Biologicals, Lawrenceville, GA), $1 \%$ antibiotic/antimycotic mixture (Sigma-Aldrich, St. Louis, MO), and bovine insulin (5 $\mu \mathrm{g} / \mathrm{mL}$ ) (Sigma-Aldrich, St. Louis, MO).

\subsubsection{In Vitro Antiproliferative Activity Assay}

The CellTiter 96® AQueous One Solution Cell Proliferation Assay was used to determine the activity of each compound (please see reference Chapter 3 Section 3.2.5 Biological Assay and Data Processing, for details).

\subsubsection{Colony Formation Assay in Soft Agar}

B16-F1 melanoma cells were plated at a colony-forming density (2000 cells per well on six-well plates) on top of $0.8 \%$ base agar. Cells were grown in $0.4 \%$ agar together with DMEM medium supplemented with fetal bovine serum and an antibioticantimycotic solution at $37^{\circ} \mathrm{C}$ in an atmosphere of $95 \%$ air and $5 \% \mathrm{CO}_{2}(93)$. Cells were treated with compounds ABI-270, -274, and -288 at different concentrations $(20,100$, and $500 \mathrm{nM}$ ). Compounds were added to the media from 1-mM DMSO stock solutions, and a corresponding dilution of DMSO was used as control. Cells were grown for 14 days. Plates were photographed, and the number of colonies was measured by Artek 880 Automated Colony Counter (Artek Systems Corporation, Farmingdale, NY).

\subsubsection{Animals}

Female C57BL/6NHsd mice, age 7-8 weeks, were obtained from Harlan Laboratories, Inc. (Indianapolis, IN). Male double homozygous SCID hairless outbred (SHO) mice, age 6-8 weeks, were obtained from Charles River Laboratories International, Inc. (Wilmington, MA). The laboratory housing the animals met all Association for Assessment and Accreditation and Laboratory Animal Care specifications. All of the 
procedures were conducted in accordance with the guidelines of our Institutional Animal Care and Use Committee.

\subsubsection{In Vivo Antitumor Study}

For C57BL/6 mice, logarithmic growth phase B16-F1 $\left(5 \times 10^{5}\right)$ cells were injected subcutaneously (s.c.) in the right dorsal flank. For SHO mice, logarithmic growth phase A375 $\left(5 \times 10^{7} / \mathrm{mL}\right)$ cells were prepared in phenyl red-free, FBS-free DMEM media and mixed at 1:1 ratio with Matrigel (BD Biosciences, San Jose, CA). Tumors were established by injecting $100 \mu \mathrm{L}$ of this mixture subcutaneously in the right dorsal flank of each mouse $\left(2.5 \times 10^{6}\right.$ cells per mouse). When the tumor reached a mean size of $100 \sim 150$

$\mathrm{mm}^{3}$, mice were randomized into 4 groups for C57BL/6 mice and 3 groups for SHO mice and treatments were started. The formulation of Tween80/Captex200 (80/20) was used to prepare the compound solution. ABI-288 at 15 and $45 \mathrm{mg} / \mathrm{kg}$ was used for C57BL $/ 6$ mice with intraperitoneal (i.p.) injection once daily for 13 days. ABI-288 at $25 \mathrm{mg} / \mathrm{kg}$ was used for SHO mice with i.p. injection once daily for 31 days. Injection volume was $30 \mu \mathrm{L}$. The control group was given only a vehicle solution injection at the same frequency. DTIC was reconstituted with sterile water according to drug usage instructions, and $100 \mu \mathrm{L}$ of this solution was given i.p. to each mouse.

Tumor volume was measured three times a week with a Traceable ${ }^{\circledR}$ electronic digital caliper (Fisher Scientific, Inc.) and calculated by using the formula $a \times b^{2} \times 0.5$, where $a$ and $b$ represented the larger and smaller diameters, respectively (94). Tumor volume was expressed as cubic millimeters. Data were expressed as mean \pm SD for each group and plotted as a function of time. Tumor growth inhibition (TGI) at the conclusion of the experiments was calculated from the formula $100-100 *[(\mathrm{~T}-\mathrm{T} 0) /(\mathrm{C}-\mathrm{C} 0)]$ where $T$ represented mean tumor volume of a treated group on a specific day, T0 represented mean tumor volume of the same group on the first day of treatment, $\mathrm{C}$ represented mean tumor volume of a control on a specific day, and $\mathrm{C} 0$ represented mean tumor volume of the same group on the first day of treatment $(95,134)$. Animal general activity and average body weight of each group were monitored during the entire experiment period to assess compound acute toxicity. The statistical significance of observed differences between growth curves was evaluated by Student's $t$ test. At the end of treatment, all mice were euthanized by $\mathrm{CO}_{2}$, and tumors were harvested for further study.

\subsubsection{Cell Cycle Analysis}

Flow cytometry analysis was performed as described elsewhere to study cell cycle phase distribution (95). A375 cells were cultured in $10 \mathrm{~cm}$ tissue culture dishes until the confluence was about $80 \%$, and then cells were treated with $0,10,50,200$, and $1000 \mathrm{nM}$ of colchicine, ABI-270, -278 , and -288 for $24 \mathrm{~h}$ in growth media. Cellular DNA was stained with PBS containing $50 \mu \mathrm{g} / \mathrm{mL}$ propidium iodide and $100 \mu \mathrm{g} / \mathrm{mL}$ RNase A. The cell cycle was determined using a BD LSR-II cytometer (BD Biosciences, San Jose, 
CA) with 10,000 cells scored. Data were analyzed and graphs were prepared using the Modfit 2.0 program (Verity Software House, Topsham, ME).

\subsubsection{In Vitro Tubulin Polymerization Assay}

Bovine brain tubulin (0.4 mg) (Cytoskeleton, Denver, CO) was mixed with 10 $\mu \mathrm{M}$ of tested compounds and incubated in $120 \mu \mathrm{L}$ of general tubulin buffer $(80 \mathrm{mM}$ PIPES, $2.0 \mathrm{mM} \mathrm{MgCl}_{2}, 0.5 \mathrm{mM}$ EGTA, pH 6.9, and $1 \mathrm{mM} \mathrm{GTP).} \mathrm{The} \mathrm{absorbance} \mathrm{of}$ wavelength at $340 \mathrm{~nm}$ was monitored every $60 \mathrm{~s}$ for $15 \mathrm{~min}$ by the SYNERGY 4 Microplate Reader (Bio-Tek Instruments, Winooski, VT). The spectrophotometer was set at $37^{\circ} \mathrm{C}$ for tubulin polymerization.

\subsubsection{Molecular Modeling}

Dr. Li selected the crystal structure of N-deacetyl-N-(2-mercaptoacetyl)colchicine (DAMA-colchicine) in tubulin complex (PDB code: 1SA0) which has been most widely used for modeling approaches (135-137). He used Schrodinger Molecular Modeling Suite 2009 (Schrodinger LLC, New York, NY) for the docking practice. ABIs were built and prepared using the Ligprep module, and they were docked into the colchicine binding site using the Glide module in Schrodinger Suite. The best docking complexes were subject to restricted molecular dynamics to release any strains by using Macromodel module with OPLS-2005 force field. The ligand and its surrounding, residues within $15 \AA$ Á were allowed to move freely, while residues outside the 15 -Á radius were kept rigid.

\subsubsection{Tubulin Colchicine Site Binding Assay}

Biotin-labeled tubulin $(0.5 \mu \mathrm{g} /$ well) (Cytoskeleton, Inc., Denver, CO) was mixed with $[3 \mathrm{H}]$ colchicine $(0.08 \mu \mathrm{M})$ (PerkinElmer LLC., Shelton, CT) and various concentrations of tested compounds in 96-well plates. The mixture was incubated at $37^{\circ} \mathrm{C}$ in binding buffer [80 mM PIPES (pH 6.8), $1 \mathrm{mM}$ EGTA, $10 \%$ glycerol, $1 \mathrm{mM} \mathrm{MgCl}_{2}$, and $1 \mathrm{mM} \mathrm{GTP}$ ] for 45 min with gentle shaking. At the end of incubation, Streptavidinlabeled SPA beads ( $80 \mu \mathrm{g} /$ well) (GE-healthcare Bio-Sciences Corp., Piscataway, NJ) were added. Signals were read using a TopCount NXT scintillation counter (PerkinElmer LLC, Shelton, CT). $\mathrm{IC}_{50}$ values (concentrations that inhibited $50 \%$ of tritiated colchicine binding) were determined by nonlinear regression analysis with GraphPad Prism (GraphPad Software, San Diego,CA).

\subsubsection{Microtubule Imaging Using Immunofluorescence Microscope}

Cellomics Cytoskeleton rearrangement kit (Thermo Scientific, Rockford, IL) was used to get a visually appreciable proof of ABIs interacting with tubulin inside the cells. 
WM-164 melanoma cells were treated with each compound for $18 \mathrm{~h}$ in duplicate using a collagen-coated 96-well plate (Becton Dickinson Labware, Bedford, MA). Then cells were fixed with 4\% paraformaldehyde (Thermo Scientific, Rockford, IL) and permeabilized using permeabilization buffer supply from the kit. Primary antibody for tubulin and fluorescence-labeled secondary antibody were subsequently added to the cells. Cell nuclei were stained by DAPI. Whole Cell Stain Green was also applied to all cells. All images were acquired with an Olympus IX71 inverted fluorescence microscope (Olympus Corp., Tokyo, Japan) with overlays from separate images of tubulin (red), nuclei (blue), and whole cells (green). For comparison, I also included paclitaxel, colchicine and ABT-751, along with ABIs.

\subsection{Results and Discussion}

\subsubsection{ABIs Have Great Potency on Melanoma Cell Lines}

I tested ABI antiproliferative activity on three melanoma cell lines: A375, B16F1, and WM-164. A375 and WM-164 are malignant human melanoma cell lines. B16-F1 is a pigment-producing mouse melanoma cell line. In order to thoroughly study the SAR for ABIs and look for the most efficacious compounds in this set, Mr. Jianjun Chen extensively modified the structure and synthesized 55 imidazole analogs. In this compound pool, I identified 29 active compounds that have $\mathrm{IC}_{50}$ values less than $10 \mu \mathrm{M}$ on the three selected melanoma cell lines. (The synthesis and SAR of ABIs will be published in another chemistry-oriented article). Twelve very active compounds had $\mathrm{IC}_{50}$ values less than $100 \mathrm{nM}$. The most active one had $\mathrm{IC}_{50} \mathrm{~s}$ less than $10 \mathrm{nM}$ on $\mathrm{A} 375$ and WM-164 cells. The structure of three representative compounds is shown in Figure 5-1. I compared our ABIs' activity with other anticancer drugs such as paclitaxel, vinblastine, colchicine, bortezomib, and ABT-751 (Table 5-1). Paclitaxel and vinblastine are clinically used anticancer drugs known to target cell tubulin $(138,139)$. Although colchicine is not an FDA-approved drug for cancer treatment, its prodrug, ZD6126, is in clinical trial for solid tumors (140). Bortezomib is the first therapeutic proteasome inhibitor and was approved in 2003 by the FDA for use in multiple myeloma (141). ABT751 is known to target the tubulin colchicine binding site. It is a promising drug candidate in clinical trial for children with relapsed or refractory neuroblastoma(142).

From our results, the three representative ABIs showed great potency on all three tested melanoma cell lines with $\mathrm{IC}_{50} \mathrm{~s}$ all less than $80 \mathrm{nM}$. The best compound, ABI-274, was even more active than paclitaxel and colchicine on A375 and WM-164 cells. Its potency was very close to that of bortezomib. Compared with ABT-751, ABI-274 was about 60 times more potent (calculated by $\mathrm{IC}_{50}$ average of three melanoma cell lines). The great in vitro potency of the ABIs suggests that they are very good candidates for melanoma treatment.

Besides their great in vitro potency, another important improvement is that ABI270 and ABI-288 do not have a trimethoxy group in their structure but retain good 
Table 5-1. In vitro cytotoxic activity of representative ABI compounds and comparison with other anticancer drugs.

\begin{tabular}{cccc}
\hline \multirow{2}{*}{$\begin{array}{c}\text { Compound } \\
\text { name }\end{array}$} & $\mathrm{A} 375$ & $\mathrm{~B} 16-\mathrm{F} 1$ & $\mathrm{WM}-164$ \\
\cline { 2 - 4 } ABI-270 & $31 \pm 5$ & $63 \pm 7$ & $28 \pm 3$ \\
ABI-274 & $9 \pm 2$ & $46 \pm 5$ & $8 \pm 2$ \\
ABI-288 & $52 \pm 4$ & $73 \pm 6$ & $74 \pm 9$ \\
Paclitaxel & $12 \pm 3$ & $17 \pm 2$ & $18 \pm 3$ \\
Vinblastine & $1 \pm 0$ & $5 \pm 1$ & $1 \pm 0$ \\
Colchicine & $20 \pm 3$ & $29 \pm 5$ & $10 \pm 2$ \\
Bortezomib & $8 \pm 1$ & $24 \pm 2$ & $8 \pm 1$ \\
ABT-751 & $1111 \pm 108$ & $2127 \pm 351$ & $661 \pm 56$ \\
\hline
\end{tabular}


activity. The lack of a trimethoxy group differentiated them chemically from previous SMART compounds and colchicine, thereby overcoming the metabolic instability drawback of the trimethoxy group.

\subsubsection{SAR Studies}

\subsubsection{SAR of Substitutions on the C Ring}

Please see Figure 5-1 for the designation of A, B, and $\mathrm{C}$ ring in $\mathrm{ABI}$ compounds. A variety of compounds (5aa-5ai) with an un-substituted A-ring and different $\mathrm{C}$ ring substituents generally showed moderate activity (Table 5-2), with $\mathrm{IC}_{50}$ values in the micromolar range (unless specified, the $\mathrm{IC}_{50}$ value for a specific compound discussed in the text is referred to as an average of all three cell lines). The most potent compound of this series was 5 aa with an average $\mathrm{IC}_{50}$ value of $160 \mathrm{nM}$. The removal of one of the methoxy groups from the 3, 4, 5-trimethoxy on the $\mathrm{C}$ ring (5ad, 5ae) led to a significant loss of activity $\left(\mathrm{IC}_{50}>10 \mu \mathrm{M}\right.$ for $5 \mathrm{ae}$ and an $\mathrm{IC}_{50}$ of $3 \mu \mathrm{M}$ for $\left.5 \mathrm{ad}\right)$. This finding is consistent with results from SMART compounds in which the 3, 4, 5-trimethoxy substituted compound was most potent. It should be noted that the compound with 4fluoro on the $\mathrm{C}$ ring (5af) also showed relatively good activity $\left(\mathrm{IC}_{50}=1 \mu \mathrm{M}\right)$, a finding that has an important implication, because replacing the trimethoxy moiety with a 4-fluoro group may provide good activity and improved metabolic stability. Interestingly, the position of the fluorine on the $\mathrm{C}$ ring was critical for activity because a shift from 4fluoro to 3-fluoro resulted in a total loss of activity $\left(\mathrm{IC}_{50}>10 \mu \mathrm{M}\right.$ for $5 \mathrm{ag}$ compared with 1 $\mu \mathrm{M}$ for $5 \mathrm{af}$ ). This result suggested that a potential hydrogen bond donor is present close to the 4-position of this ring. As shown in the molecular modeling studies below, this hydrogen bond donor is likely to be the thiol group in Cys-241 in loop 7 of the $\beta$-subunit in $\alpha / \beta$-tubulin dimer.

Other substituents such as methoxy and methyl at the 3 or 4 position on the $\mathrm{C}$ ring (5ab, 5ac, 5ah, 5ai) were also evaluated, but none showed good activity $\left(\mathrm{IC}_{50}>10 \mu \mathrm{M}\right)$. As clearly indicated in Table 5-2, the positions of the $\mathrm{A}$ and $\mathrm{C}$ rings were critical. A simple shift of the $\mathrm{C}$ ring moiety from position 4 to position 1 in the imidazole ring ( $\mathrm{B}$ ring resulted in total loss of activity ( $\mathrm{IC}_{50}>10 \mu \mathrm{M}$ for 5aba, 5aaa, 3a, 3x, 3j). This result is consistent with recent reports in which the position of the aryl group was found to be important for antiproliferative activity $(143,144)$. Bellina and co-workers reported potent antitumor activity for a series of 1, 5- and 1, 2-diaryl- $1 H$-imidazole analogs. While the 1 , 5-diaryl-imidazole analogs have nanomolar activity, a simple shift of the diaryl substitution from the 1,5-position to 1,2-position resulted in significantly lower activity down to the micromolar range (143). Similarly, Wang and co-workers reported that 4, 5disubstituted and 1, 5-disubstituted imidazoles are much more active than the corresponding 1, 2-disubstituted imidazoles (144). 
Table 5-2. In vitro cytotoxic activity of $\mathrm{ABI}$ compounds with $\mathrm{B}$ and $\mathrm{C}$ ring modification.

\begin{tabular}{|c|c|c|c|c|c|}
\hline \multirow{2}{*}{ Structure } & \multirow{2}{*}{ ID } & \multirow{2}{*}{$\mathrm{R}$} & \multicolumn{3}{|c|}{$\mathrm{IC}_{50} \pm \operatorname{SEM}(\mu \mathrm{M})(\mathrm{n}=3)$} \\
\hline & & & A375 & B16-F1 & WM-164 \\
\hline \multirow{15}{*}{$0=$} & & & $200 \pm 80$ & $100 \pm 12$ & $100 \pm 25$ \\
\hline & $5 a a$ & $3,4,5-(\mathrm{OMe})_{3}$ & $\mathrm{nM}$ & $\mathrm{nM}$ & $\mathrm{nM}$ \\
\hline & $5 \mathbf{a b}$ & 4-OMe & $>10$ & $>10$ & $>10$ \\
\hline & 5 ac & 3- OMe & $>10$ & $>10$ & $>10$ \\
\hline & 5 ad & $3,5-(\mathrm{OMe})_{2}$ & $3 \pm 1$ & $5 \pm 1$ & $2 \pm 0$ \\
\hline & 5 ae & $3,4-(\mathrm{OMe})_{2}$ & $>10$ & $>10$ & $>10$ \\
\hline & 5 af & 4-F & $1 \pm 0$ & $1 \pm 0$ & $1 \pm 0$ \\
\hline & $5 \mathrm{ag}$ & $3-\mathrm{F}$ & $>10$ & $>10$ & $>10$ \\
\hline & $5 \mathbf{a h}$ & 4-Me & $>10$ & $>10$ & $>10$ \\
\hline & $5 \mathbf{a i}$ & 3-Me & $>10$ & $>10$ & $>10$ \\
\hline & 5 aba & 4-OMe & $>10$ & $>10$ & $>10$ \\
\hline & 5aaa & $3,4,5-(\mathrm{OMe})_{3}$ & $>10$ & $>10$ & $>10$ \\
\hline & $3 \mathbf{a}$ & $\mathrm{H}$ & $>10$ & $>10$ & $>10$ \\
\hline & $3 x$ & $4-\mathrm{NO}_{2}$ & $>10$ & $>10$ & $>10$ \\
\hline & $3 \mathbf{j}$ & 4-OBn & $>10$ & $>10$ & $>10$ \\
\hline
\end{tabular}




\subsubsection{SAR of Substitutions on the A Ring}

Because compounds with 3, 4, 5-trimethoxy and 4-fluoro substitutions on the C ring showed good activity, a series of compounds was synthesized with fixed substitutions on the $\mathrm{C}$ ring (4-fluoro or 3, 4, 5-trimethoxy) and different substitutions on the A ring (Table 5-3). These compounds demonstrated excellent antiproliferative activity with $\mathrm{IC}_{50}$ values as low as $8 \mathrm{nM}$ on WM-164 cell line (5da). In general, compounds incorporating a single substituent on the para-position of the A ring were more potent as can be seen from the activities of $5 \mathrm{ca}, 5 \mathrm{cb}, 5 \mathrm{da}, 5 \mathrm{db}, 5 \mathrm{fa}, 5 \mathrm{fb}, 5 \mathrm{ga}$, and $5 \mathrm{gb}\left(\mathrm{IC}_{50}=8 \sim 110 \mathrm{nM}\right) .5 \mathrm{db}-\mathrm{HCl}$ salt $\left(\mathrm{IC}_{50}=172 \mathrm{nM}\right)$ showed slightly diminished activity compared with the corresponding free base $5 \mathrm{db}\left(\mathrm{IC}_{50}=109 \mathrm{nM}\right)$. Compound $5 \mathrm{fb}\left(\mathrm{IC}_{50}=64\right.$ $\mathrm{nM}$ ), with a single halogen substituent in the para-position of the $\mathrm{A}$ and $\mathrm{C}$ rings, demonstrated potent and was devoid of a methoxy moiety. Compounds with 3, 4, 5 trimethoxy substituents on the A ring lost activity completely $\left(\mathrm{IC}_{50}>10 \mu \mathrm{M}\right.$ for $\left.5 \mathrm{ea}, 5 \mathrm{eb}\right)$, suggesting very different binding environments near the $\mathrm{A}$ ring and $\mathrm{C}$ ring. Removal of

the 5-methoxy substituent from the A-ring improved activity significantly $\left(\mathrm{IC}_{50}=330 \mathrm{nM}\right.$ and $>10 \mu \mathrm{M}$ for 5ha, 5ea respectively). Demethylation of the 3, 4, 5-trimethoxy decreased activity sharply from $43 \mathrm{nM}(5 \mathrm{fa})$ to $4 \mu \mathrm{M}$ (6fa). Similar results were observed for $6 \mathrm{ea}, 5 \mathrm{ka}, 5 \mathrm{~kb}$, and $6 \mathrm{ha}$ due to the demethylation of subsituents on either the A or C ring. Electron-donating groups (4-methoxy, 4-dimethylamino, 4-methyl) and electronwithdrawing groups (4-chloro, 2-trifluoromethyl) on the A ring did not show substantial differences in activity. The introduction of a trifluoromethyl group at the ortho position of the A ring caused complete loss of activity $\left(\mathrm{IC}_{50}>10 \mu \mathrm{M}\right.$ for $\left.5 \mathrm{ia}, 5 \mathrm{ib}\right)$. The presence of a benzoloxy group at the para position of $A$ ring $\left(\mathrm{IC}_{50}=75 \mathrm{nM}\right.$ for $5 \mathrm{jb}$ ) resulted in a 440 fold increase in activity when compared with the para-hydroxy compound $5 \mathrm{~kb}\left(\mathrm{IC}_{50}=33\right.$ $\mu \mathrm{M})$. It is worthwhile to note that compound $5 \mathrm{jb}$, with the 4-fluoro in the $\mathrm{C}$ ring, has better activity than does its counterpart $5 \mathrm{ja}$, which has a 3, 4, 5-trimethoxy group in the $\mathrm{C}$ ring $\left(\mathrm{IC}_{50}\right.$ is $75 \mathrm{nM}$ for $5 \mathrm{jb}$, and $7 \mu \mathrm{M}$ for $\left.5 \mathrm{ja}\right)$.

\subsubsection{SAR of Additional Substitutions on the B Ring}

To our surprise, some of the compounds with a phenylsulfonyl protection group attached to the nitrogen of the imidazole ring (4cb, 4db, 4fb, 4ga, 4gb, 4ha, 4jb) were also very active with $\mathrm{IC}_{50}$ in the nanomolar range (Table 5-4). Generally the activities of these compounds are comparable to their corresponding unprotected counterparts as exemplified by comparing the activities of $4 \mathrm{cb}(43 \mathrm{nM}), 4 \mathrm{db}(111 \mathrm{nM}), 4 \mathrm{fb}(72 \mathrm{nM}), 4 \mathrm{ga}$ (285 $\mathrm{nM}), 4 \mathrm{gb}(87 \mathrm{nM})$, 4ha $(268 \mathrm{nM})$, and $4 \mathrm{jb}(61 \mathrm{nM})$ with their corresponding unprotected counterparts $5 \mathrm{cb}(36 \mathrm{nM}), 5 \mathrm{db}(109 \mathrm{nM}), 5 \mathrm{fb}(64 \mathrm{nM}), 5 \mathrm{ga}(131 \mathrm{nM}), 5 \mathrm{gb}$ (72 nM), 5ha (330 nM), and 5jb (75 nM). Other compounds (4ab-4ag, 4ea, 4eb, 4hb, 4ia, and 4ib, $1-50 \mu \mathrm{M})$ were generally much less active, also in line with their counterparts (5ab-5ag, 5ea, 5eb, 5hb, 5ia, and 5ib, 1-50 $\mu \mathrm{M}$ ). 
Table 5-3. In vitro cytotoxic activity of ABI compounds with A ring modification.

\begin{tabular}{|c|c|c|c|c|c|c|}
\hline \multirow{2}{*}{ Structure } & \multirow{2}{*}{ ID } & \multirow{2}{*}{$\mathrm{R}^{1}$} & \multirow{2}{*}{$\mathrm{R}^{2}$} & \multicolumn{3}{|c|}{$\mathrm{IC}_{50} \pm \operatorname{SEM}(\mathrm{nM})(\mathrm{n}=3)$} \\
\hline & & & & A375 & B16-F1 & WM-164 \\
\hline \multirow{24}{*}{$\underbrace{O=}_{\substack{\mathrm{R}^{2}\\
}=1}$} & $5 \mathbf{b a}$ & $4-F$ & $3,4,5-(\mathrm{OMe})_{3}$ & $205 \pm 19$ & $320 \pm 41$ & $73 \pm 8$ \\
\hline & $5 \mathrm{ca}$ & 4-OMe & $3,4,5-(\mathrm{OMe})_{3}$ & $30 \pm 5$ & $108 \pm 12$ & $31 \pm 4$ \\
\hline & $5 \mathrm{cb}$ & 4-OMe & $4-\mathrm{F}$ & $31 \pm 5$ & $63 \pm 7$ & $28 \pm 3$ \\
\hline & $5 \mathrm{da}$ & 4-Me & $3,4,5-(\mathrm{OMe})_{3}$ & $9 \pm 2$ & $46 \pm 5$ & $8 \pm 2$ \\
\hline & $5 \mathrm{db}$ & 4-Me & $4-\mathrm{F}$ & $142 \pm 13$ & $222 \pm 10$ & $156 \pm 19$ \\
\hline & \multicolumn{3}{|c|}{ 5db-HCl } & $108 \pm 11$ & $297 \pm 23$ & $112 \pm 9$ \\
\hline & 5ea & $3,4,5-(\mathrm{OMe})_{3}$ & $3,4,5-(\mathrm{OMe})_{3}$ & 4800 & $>10000$ & $>10000$ \\
\hline & $5 \mathrm{eb}$ & $3,4,5-(\mathrm{OMe})_{3}$ & $4-\mathrm{F}$ & $>10000$ & $>10000$ & $>10000$ \\
\hline & $5 f a$ & 4-Cl & $3,4,5-(\mathrm{OMe})_{3}$ & $43 \pm 5$ & $168 \pm 14$ & $26 \pm 3$ \\
\hline & $5 \mathrm{fb}$ & $4-\mathrm{Cl}$ & $4-\mathrm{F}$ & $52 \pm 4$ & $73 \pm 6$ & $74 \pm 9$ \\
\hline & $6 f a$ & $4-\mathrm{Cl}$ & $3,4,5-(\mathrm{OH})_{3}$ & 3900 & 1810 & 2100 \\
\hline & 5ga & $4-\mathrm{N}(\mathrm{Me})_{2}$ & $3,4,5-(\mathrm{OMe})_{3}$ & $82 \pm 9$ & $361 \pm 29$ & $80 \pm 11$ \\
\hline & $5 \mathrm{gb}$ & $4-\mathrm{N}(\mathrm{Me})_{2}$ & $4-\mathrm{F}$ & $56 \pm 7$ & $129 \pm 11$ & $62 \pm 8$ \\
\hline & 5 ha & $3,4-(\mathrm{OMe})_{2}$ & $3,4,5-(\mathrm{OMe})_{3}$ & $113 \pm 14$ & $1400 \pm 200$ & $191 \pm 18$ \\
\hline & $5 \mathrm{hb}$ & $3,4-(\mathrm{OMe})_{2}$ & $4-\mathrm{F}$ & 10000 & 4210 & 1400 \\
\hline & 5 ia & $2-\mathrm{CF}_{3}$ & $3,4,5-(\mathrm{OMe})_{3}$ & $>10000$ & $>10000$ & $>10000$ \\
\hline & $5 \mathrm{ib}$ & $2-\mathrm{CF}_{3}$ & $4-\mathrm{F}$ & $>10000$ & $>10000$ & $>10000$ \\
\hline & 6ea & $3,4,5-(\mathrm{OH})_{3}$ & $3,4,5-(\mathrm{OH})_{3}$ & $>10000$ & $>10000$ & $>10000$ \\
\hline & $5 \mathbf{j a}$ & 4-OBn & $3,4,5-(\mathrm{OMe})_{3}$ & 5200 & 10000 & 5500 \\
\hline & $5 \mathbf{j b}$ & 4-OBn & 4-F & $93 \pm 8$ & $117 \pm 16$ & $90 \pm 12$ \\
\hline & $5 \mathrm{ka}$ & $4-\mathrm{OH}$ & $3,4,5-(\mathrm{OMe})_{3}$ & 1600 & 2400 & 1800 \\
\hline & $5 \mathbf{k b}$ & 4-OH & $4-\mathrm{F}$ & 10000 & $>10000$ & $>10000$ \\
\hline & 6ha & $3,4-(\mathrm{OH})_{2}$ & $3,4,5-(\mathrm{OH})_{3}$ & $>10000$ & $>10000$ & $>10000$ \\
\hline & \multicolumn{3}{|c|}{ Colchicine } & $20 \pm 3$ & $29 \pm 5$ & $10 \pm 2$ \\
\hline
\end{tabular}


Table 5-4. In vitro cytotoxic activity of ABI compounds with protection on B ring.

\begin{tabular}{|c|c|c|c|c|c|c|}
\hline \multirow{2}{*}{ Structure } & \multirow{2}{*}{ ID } & \multirow{2}{*}{$\mathrm{R}^{1}$} & \multirow{2}{*}{$\mathrm{R}^{2}$} & \multicolumn{3}{|c|}{$\mathrm{IC}_{50} \pm \operatorname{SEM}(\mathrm{nM})(\mathrm{n}=3)$} \\
\hline & & & & A375 & B16-F1 & WM-164 \\
\hline & $4 a b$ & $\mathrm{H}$ & 4-OMe & $>10000$ & $>10000$ & $>10000$ \\
\hline & $4 a c$ & $\mathrm{H}$ & 3-OMe & $>10000$ & $>10000$ & $>10000$ \\
\hline & 4ah & $\mathrm{H}$ & 4-Me & $>10000$ & $>10000$ & $>10000$ \\
\hline & 4af & $\mathrm{H}$ & $4-\mathrm{F}$ & $630 \pm 72$ & $946 \pm 86$ & $596 \pm 61$ \\
\hline & 4ag & $\mathrm{H}$ & $3-\mathrm{F}$ & $>10000$ & $>10000$ & $>10000$ \\
\hline & $4 \mathrm{cb}$ & 4-OMe & $4-\mathrm{F}$ & $36 \pm 5$ & $71 \pm 8$ & $43 \pm 6$ \\
\hline & $4 d b$ & 4-Me & $4-\mathrm{F}$ & $113 \pm 14$ & $287 \pm 31$ & $107 \pm 14$ \\
\hline & 4ea & $3,4,5-(\mathrm{OMe})_{3}$ & $3,4,5-(\mathrm{OMe})_{3}$ & $>10000$ & $>10000$ & $>10000$ \\
\hline & $4 \mathrm{eb}$ & $3,4,5-(\mathrm{OMe})_{3}$ & $4-\mathrm{F}$ & 3840 & $>10000$ & $>10000$ \\
\hline & $4 f b$ & 4-Cl & 4-F & $88 \pm 9$ & $107 \pm 12$ & $70 \pm 6$ \\
\hline & 4ga & $4-\mathrm{N}(\mathrm{Me})_{2}$ & $3,4,5-(\mathrm{OMe})_{3}$ & $162 \pm 13$ & $1200 \pm 90$ & $308 \pm 32$ \\
\hline & 4gb & $4-\mathrm{N}(\mathrm{Me})_{2}$ & $4-\mathrm{F}$ & $55 \pm 7$ & $242 \pm 26$ & $56 \pm 4$ \\
\hline & 4ha & $3,4-(\mathrm{OMe})_{2}$ & $3,4,5-(\mathrm{OMe})_{3}$ & $192 \pm 15$ & $970 \pm 68$ & $139 \pm 15$ \\
\hline & $4 \mathrm{hb}$ & $3,4-(\mathrm{OMe})_{2}$ & $4-F$ & $960 \pm 59$ & $2000 \pm 400$ & $1400 \pm 30$ \\
\hline & $4 \mathbf{i a}$ & $2-\mathrm{CF}_{3}$ & $3,4,5-(\mathrm{OMe})_{3}$ & $>10000$ & $>10000$ & $>10000$ \\
\hline & $4 i b$ & $2-\mathrm{CF}_{3}$ & $4-\mathrm{F}$ & $>10000$ & $>10000$ & $>10000$ \\
\hline & $4 \mathrm{jb}$ & $4-\mathrm{OBn}$ & $4-\mathrm{F}$ & $64 \pm 7$ & $110 \pm 15$ & $48 \pm 5$ \\
\hline
\end{tabular}




\subsubsection{ABIs Overcome Multi-Drug Resistance on Drug Resistant Melanoma Cells}

Drug resistance is the major cause of cancer chemotherapy failure. One major contributor to multi-drug resistance is overexpression of P-glycoprotein (P-gp). This protein is a clinically important transporter protein belonging to the ATP-binding cassette family of cell membrane transporters. It can pump substrates including anticancer drugs out of tumor cells through an ATP-dependent mechanism (145). To test whether our ABIs could overcome P-gp-mediated multi-drug resistance, Dr. Li obtained one P-gp overexpressing human melanoma cell line, MDA-MB-435/LCCMDR1, and the matching sensitive parent cell line from Dr. Robert Clarke at Georgetown University. I then tested three representative compounds ABI-270, -274 , and -288 on these two cell lines together with colchicine, paclitaxel, and vinblastine. The dose response curves are shown in Figure 5-2. The $\mathrm{IC}_{50}$ values and calculated resistance index are shown in Table 5-5. Big distance between two curves for paclitaxel, vinblastine, and colchicine means they were substrates for P-gp. The two curves of each ABI compound overlapped means they were not substrates for P-gp and overcame multi-drug resistance.

The results clearly showed that cell line MDA-MB-435/LCCMDR1 was very resistant to colchicine, paclitaxel, and vinblastine. But our ABIs showed equal potency to the drug resistant cell line and the sensitive parent cell line. This result strongly suggests that ABIs are not substrate for P-gp. Thus, they overcame the multi-drug resistance found in MDA-MB-435/LCCMDR1 cells. Note that ABI-274 has a resistance index of 3, meaning that MDA-MB-435/LCCMDR1 cells have some resistance to this compound. I believe that the reason may be that ABI-274 has a trimethoxy-phenyl group, which makes it partially chemically similar to colchicine.

\subsubsection{ABIs Inhibit Melanoma Colony Formation in Soft Agar}

Colony formation assay has been generally used to measure the sensitivity of tumors to anticancer drugs. Results reflect the therapeutic efficacy of a drug (146). To further measure ABIs' anti-melanoma efficacy, I tested ABI-270, -274, and -288 on melanoma colony formation in soft agar. Three different concentrations - 20, 100, 500 $\mathrm{nM}$ - were tested. Representative photos are shown in Figure 5-3. Quantified results are shown in Figure 5-4. After 14 days of incubation, about 130 detectable colonies (diameter larger than $100 \mu \mathrm{m}$ ) were formed in controls (no treatment). Compounds ABI270 and -274 effectively inhibited B16-F1 melanoma colony formation even at the lowest tested concentration, $20 \mathrm{nM}$ ( $\mathrm{p}<0.05$ compared with control). ABI-288 showed effective inhibition at $100 \mathrm{nM}$. All three tested compounds showed complete inhibition of colony formation at $0.5 \mu \mathrm{M}$, further proving ABIs' anti-melanoma efficacy.

\subsubsection{ABIs Inhibited Melanoma Tumor Growth In Vivo}

Encouraged by ABIs' good activity against melanoma cells and inhibition of melanoma colony formation in soft agar, in vivo anti-melanoma activity of ABIs was 

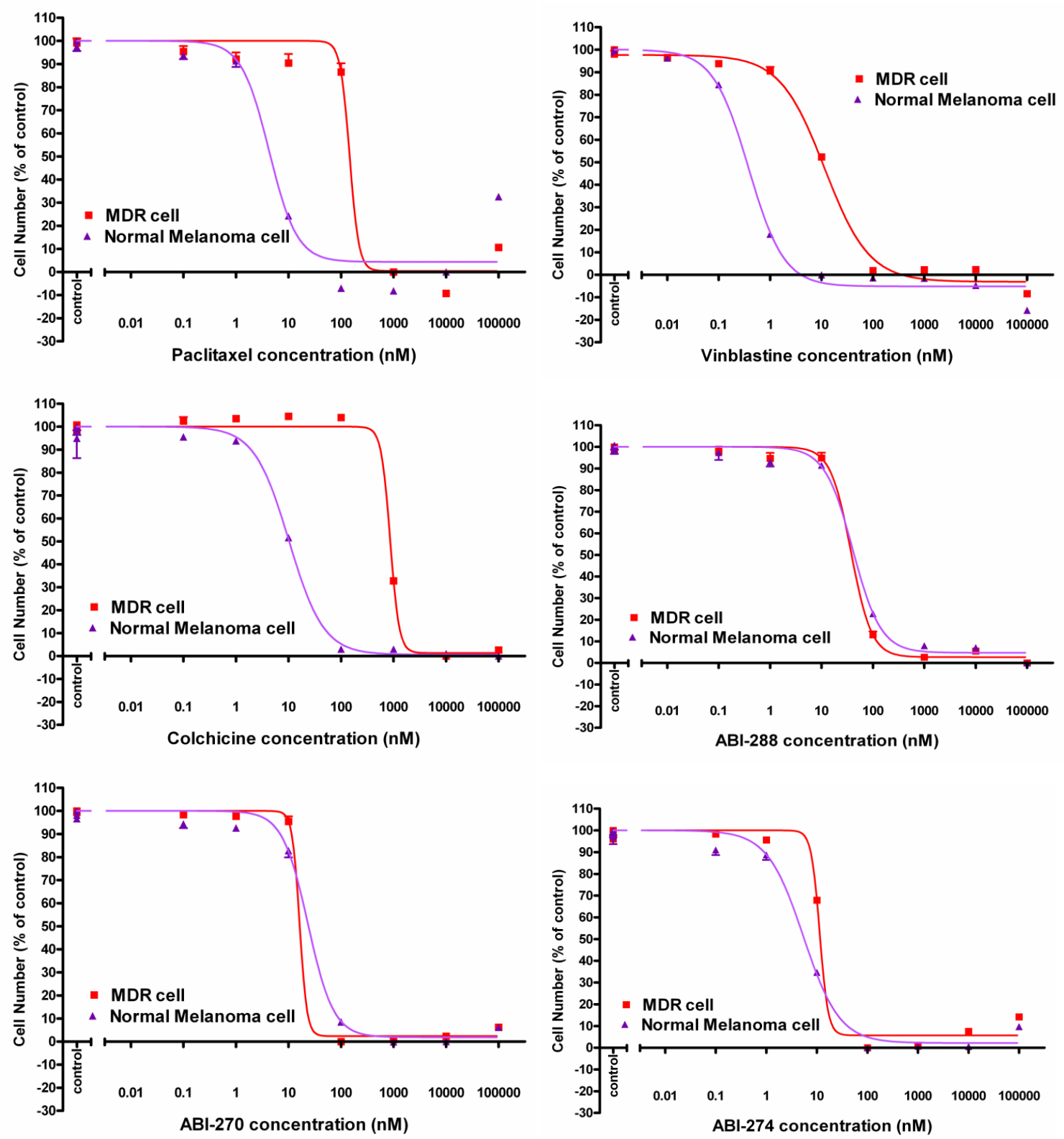

Figure 5-2. Dose-response curves showed ABI compounds overcome multi-drug resistance for melanoma compared with other anticancer drugs. 
Table 5-5. In vitro cytotoxic activity comparison of ABI compounds with other anticancer drugs on multi-drug resistant melanoma cell line.

\begin{tabular}{cccc}
\hline \multirow{2}{*}{$\begin{array}{c}\text { Compound } \\
\text { name }\end{array}$} & \multicolumn{2}{c}{$\mathrm{IC}_{50} \pm \mathrm{SEM}(\mathrm{nM})(\mathrm{n}=3)$} & $\begin{array}{c}\text { Resistance index } \\
(\mathrm{R})^{*}\end{array}$ \\
\cline { 2 - 3 } & MDA-MB-435 & $\begin{array}{c}\text { MDA-MB-435 } \\
\text { /LCC6MDR1 }\end{array}$ & 66 \\
\hline Colchicine & $10 \pm 1$ & $658 \pm 50$ & 69 \\
Paclitaxel & $4 \pm 1$ & $277 \pm 41$ & 28 \\
Vinblastine & $400 \pm 100 \mathrm{pM}$ & $11 \pm 1$ & 3 \\
ABI-274 & $5 \pm 1$ & $15 \pm 2$ & 1 \\
ABI-270 & $24 \pm 2$ & $30 \pm 4$ & 1 \\
ABI-288 & $41 \pm 2$ & $38 \pm 2$ & 66 \\
\hline
\end{tabular}

* Resistance indexes were calculated by dividing $\mathrm{IC}_{50}$ values on multi-drug resistant cell line MDA-MB-435/LCC6MDR1 by $\mathrm{IC}_{50}$ values on the matching sensitive parent cell line MDA-MB-435. 


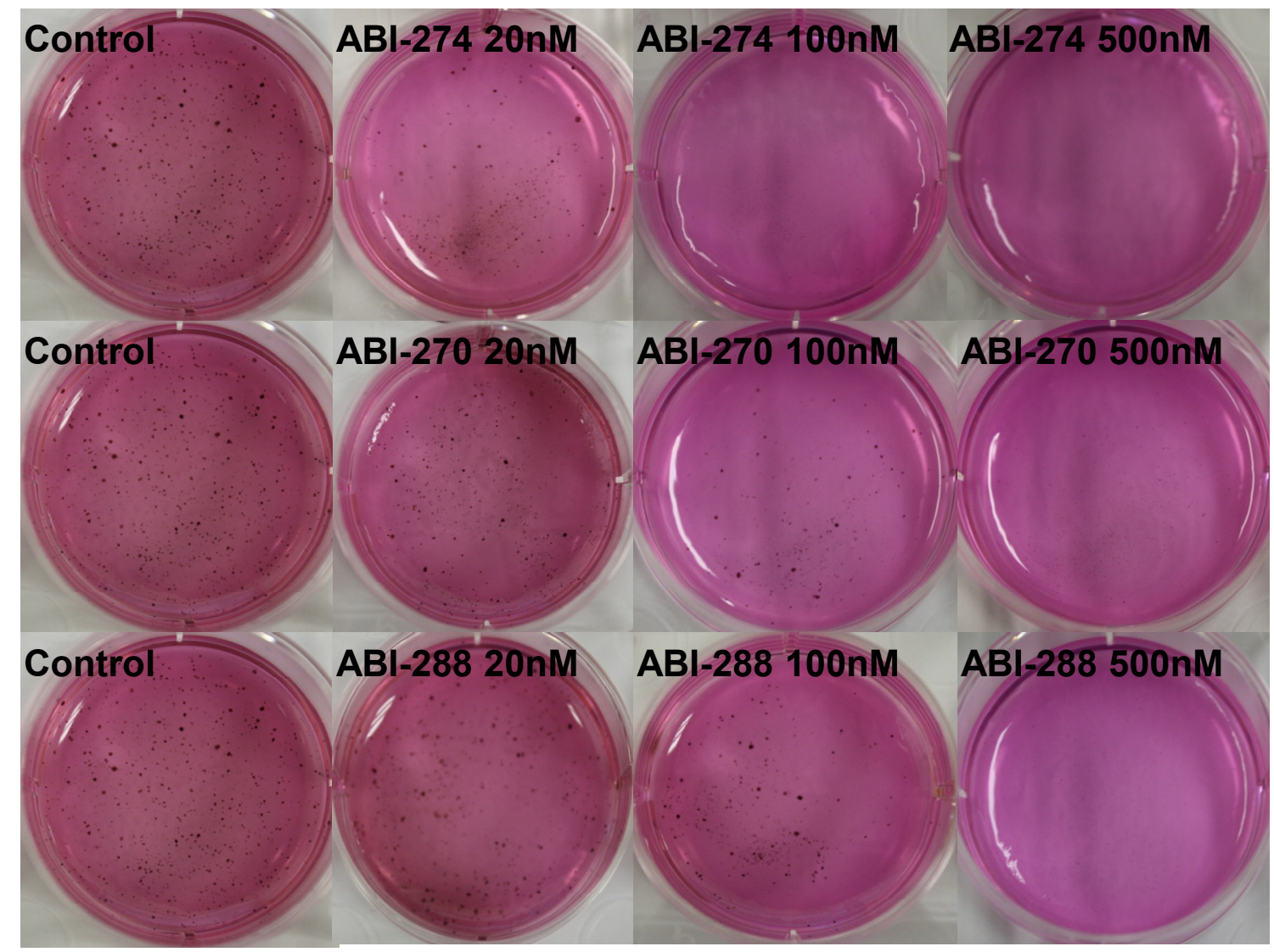

Figure 5-3. B16-F1 melanoma colony formation assay in soft agar for ABI compounds. Compounds ABI-270 and 274 effectively inhibited B16-F1 melanoma colony formation even at the lowest tested concentration $20 \mathrm{nM}$ ( $\mathrm{p}<0.05$ compared with control). ABI-288 showed effective inhibition at $100 \mathrm{nM}$. All three tested compounds showed complete inhibition for colony formation at $0.5 \mu \mathrm{M}$. 

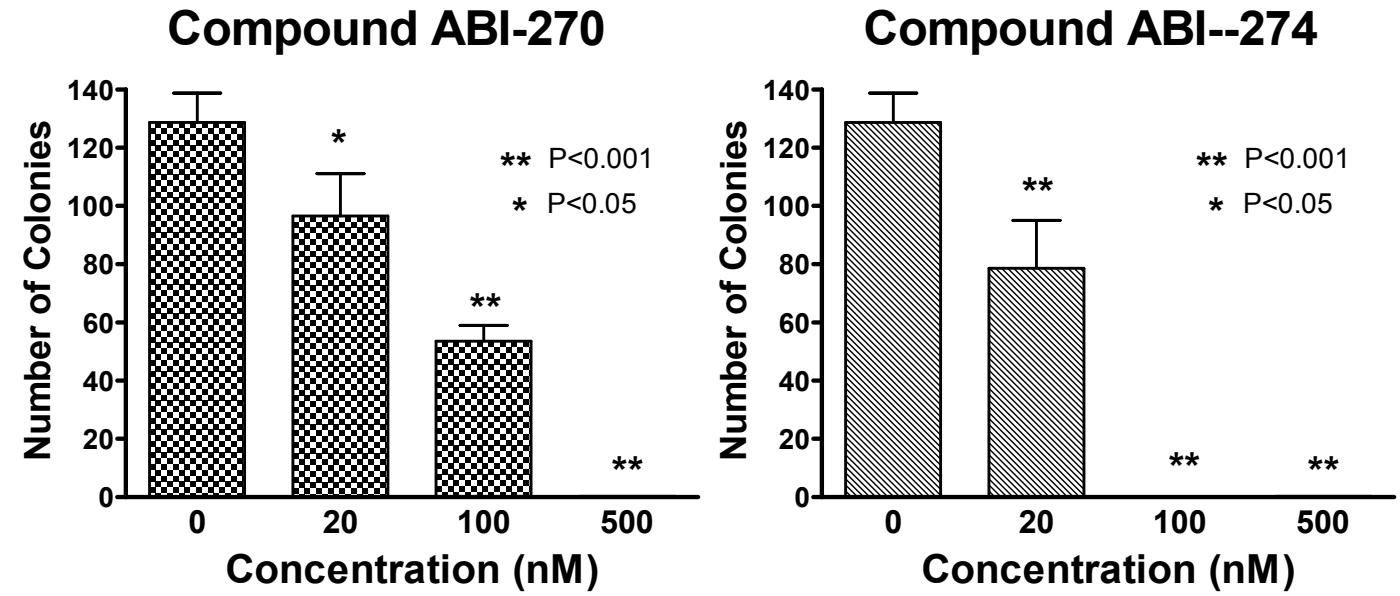

Compound $\mathrm{ABI}-288$

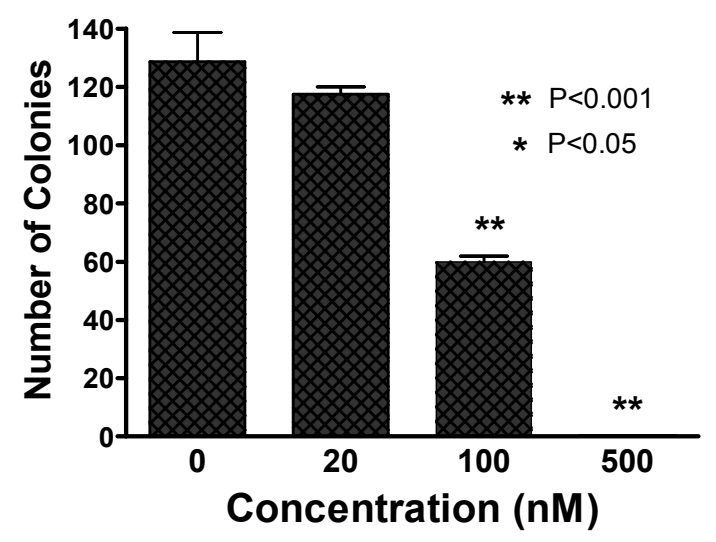

Figure 5-4. Quantified representation of colony formation assay using colony counter. ${ }^{*} p<0.05$ compared with control. ${ }^{* *} p<0.001$ compared with control. 
tested. Compound ABI-288 was chosen because it does not have a trimethoxy group and its solubility is better than that of the others. Based on our previous study experience, two dose levels, 15 and $45 \mathrm{mg} / \mathrm{kg}$, were chosen. DTIC at $60 \mathrm{mg} / \mathrm{kg}$ was used as a positive control according to the literature (109). B16-F1 melanoma allograft model on C57BL/6 mice was first chosen for study. After 13 days of treatment (Figure 5-5), ABI-288 inhibited melanoma tumor growth (TGI value) by $32 \%$ at $15 \mathrm{mg} / \mathrm{kg}$ and $82 \%$ at $45 \mathrm{mg} / \mathrm{kg}$. Student's $t$ test p value of ABI-288 at $45 \mathrm{mg} / \mathrm{kg}$ compared with control was less than 0.001 , indicating a significant difference. The $t$ test $\mathrm{p}$ value of ABI-288 at $15 \mathrm{mg} / \mathrm{kg}$ compared with control was 0.08 , suggesting that this dose was not effective. Comparing ABI-288 at $45 \mathrm{mg} / \mathrm{kg}$ with DTIC at $60 \mathrm{mg} / \mathrm{kg}$, which had a TGI of $51 \%$, the $t$ test p value was about 0.001 , suggesting that ABI-288 had substantially better activity than did DTIC. I also observed a continuous average body weight loss in the ABI-288 $45 \mathrm{mg} / \mathrm{kg}$ treatment group. For the control and ABI-288 $15 \mathrm{mg} / \mathrm{kg}$ treatment groups, average body weight increased slightly throughout the experiment period.

To further confirm ABIs' in vivo activity, A375 human melanoma xenograft model on SHO mice was used, and ABI-288 at $25 \mathrm{mg} / \mathrm{kg}$ was tested. DTIC at a dose of $60 \mathrm{mg} / \mathrm{kg}$ was used as a positive control again. After 31 days of treatment (Figure 5-6), ABI-288 inhibited melanoma tumor growth (TGI value) by $69 \%$, whereas DTIC inhibited growth by $52 \%$. The $t$ test p value of ABI-288 treatment versus control was less than 0.001 , suggesting that ABI-288 significantly inhibited melanoma tumor growth at 25 $\mathrm{mg} / \mathrm{kg}$. The $t$ test $\mathrm{p}$ value of ABI-288 treatment versus DTIC was less than 0.05 , suggesting again that ABI-288 had better activity than did DTIC. Average body weight of all groups increased slightly throughout the experiment period. Physical activities for the mice also looked normal, suggesting that $25 \mathrm{mg} / \mathrm{kg}$ was a well tolerated dose for SHO mice.

\subsubsection{ABIs Target Cell Tubulin at the Colchicine Binding Site}

The cellular target of ABIs was determined by the following five experiments.

\subsubsection{Cell Cycle Analysis}

After identifying the great anti-melanoma potency of ABIs and their capability for overcoming multi-drug resistance, our next goal was to determine the cellular target of this class of compounds. Based on their high potency and behavior similarity with other tubulin binding agents on melanoma cells, I hypothesized that these compounds may also target tubulin. If our hypothesis were right, ABIs should arrest cells in the G2/M phase, so I did a cell cycle analysis. I tested ABI-270, -274, and -288 together with colchicine as a positive control on A375 cells. Four different concentrations - 10, 50, 200, and $1000 \mathrm{nM}$ - of each compound were chosen to show the dose effect (Figure 5-7 and Figure 5-8). For controls (no treatment) without interference, about $16 \%$ of A375 cells were distributed in the $\mathrm{G} 2 / \mathrm{M}$ phase. For the colchicine treatment group, as concentration increased from $10 \mathrm{nM}$ to $50 \mathrm{nM}$, the percentage of cells distributed in the $\mathrm{G} 2 / \mathrm{M}$ phase 


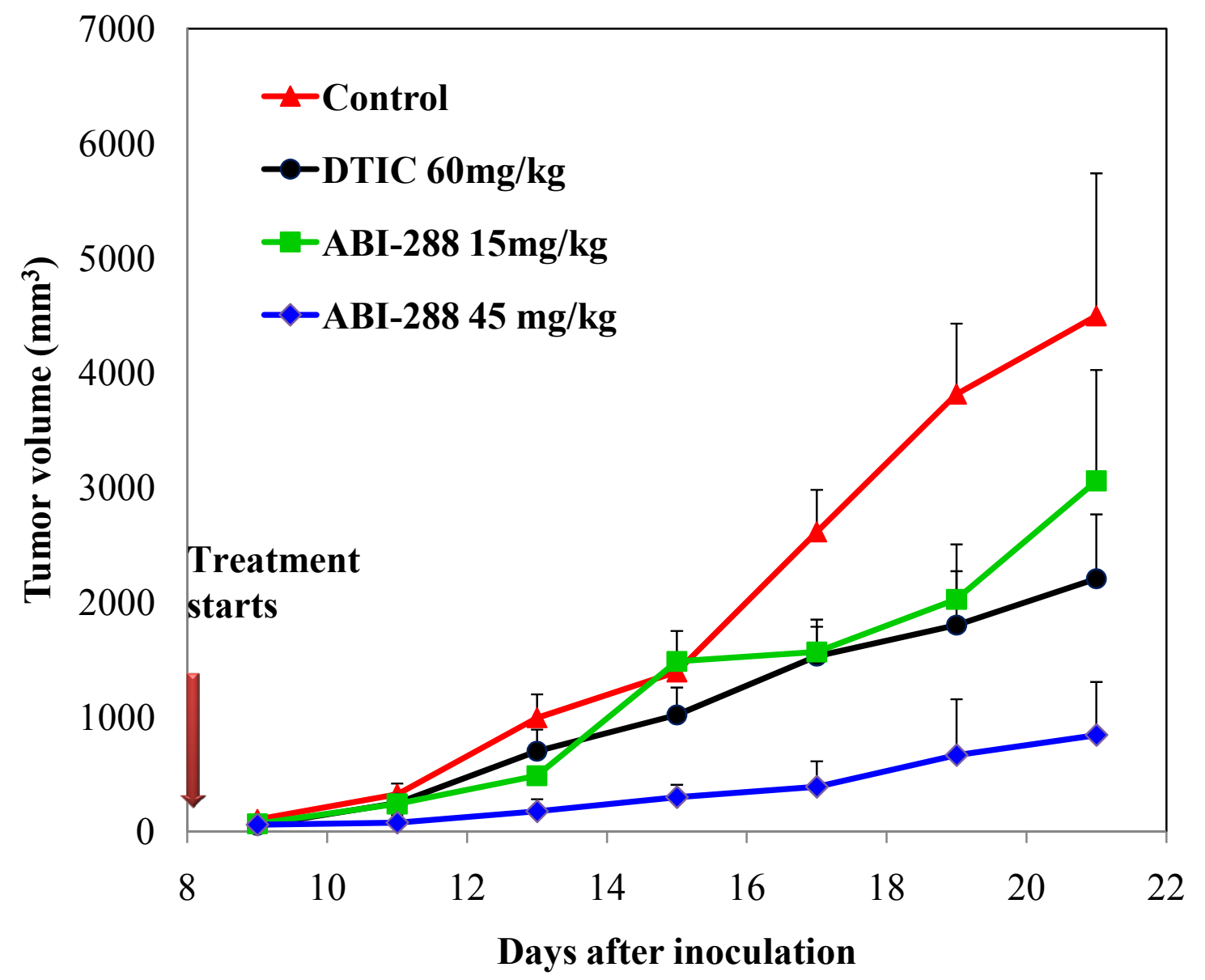

Figure 5-5. In vivo studies on C57BL/6 mice bearing B16-F1 melanoma allograft for ABI-288.

ABI-288 inhibited melanoma tumor growth in a dose-dependent manner. Each mouse received $0.5 \times 10^{6}$ cells by s.c. injection into the flank. $30 \mu \mathrm{L}$ i.p. daily treatments were started when tumor size reached $\sim 100 \mathrm{~mm}^{3}$. Control, vehicle solution only; points, means; bars, SD. $\mathrm{n}=5$ per group. 


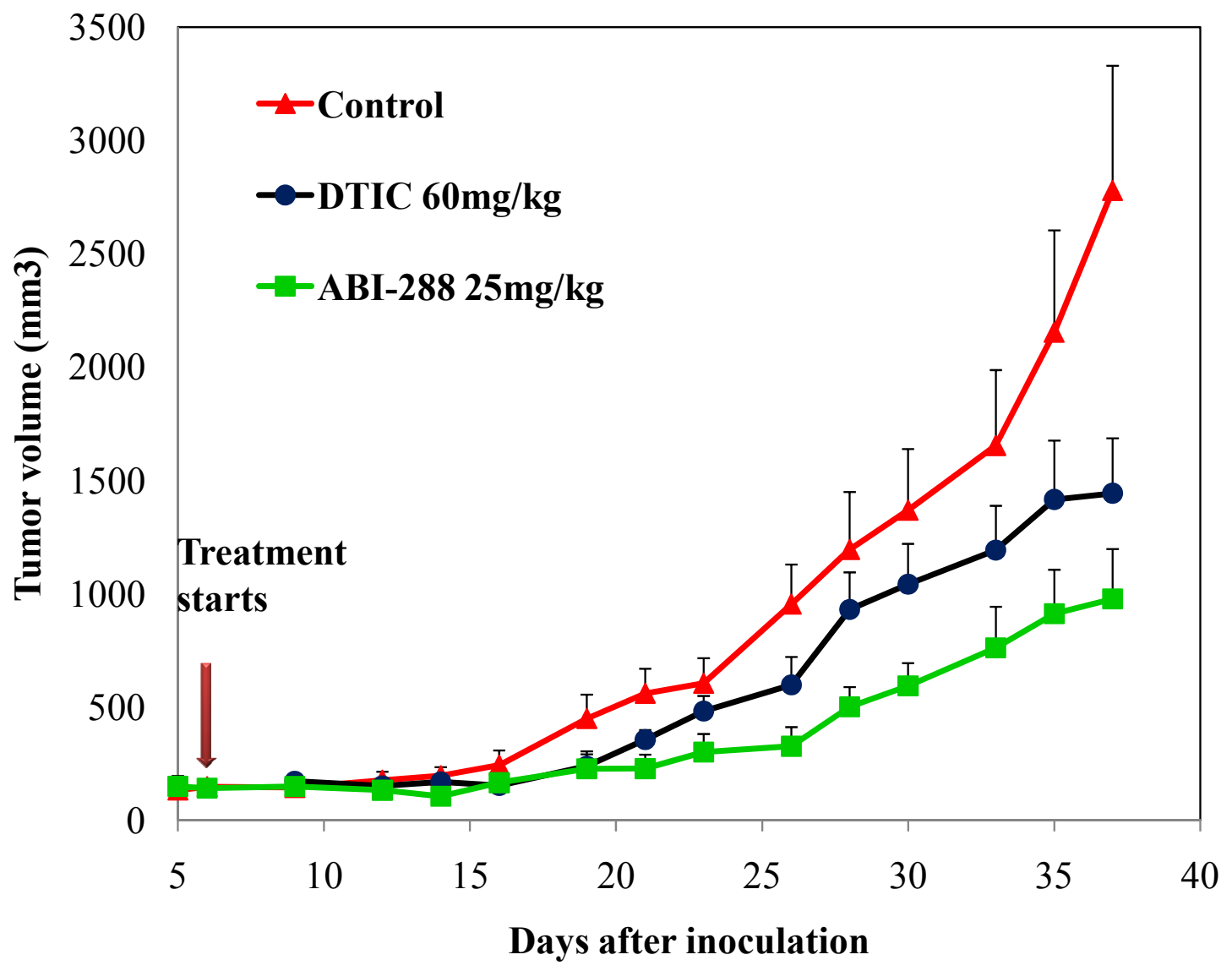

Figure 5-6. In vivo studies on $\mathrm{SHO}$ nude mice bearing A375 human melanoma xenograft for ABI-288.

ABI-288 inhibited melanoma tumor growth in a dose-dependent manner. Each mouse received $2.5 \times 10^{6}$ cells by s.c. injection into the flank. $30 \mu \mathrm{L}$ i.p. daily treatments were started when tumor size reached $\sim 150 \mathrm{~mm}^{3}$. Control, vehicle solution only; points, means; bars, SD. $\mathrm{n}=5$ per group. 

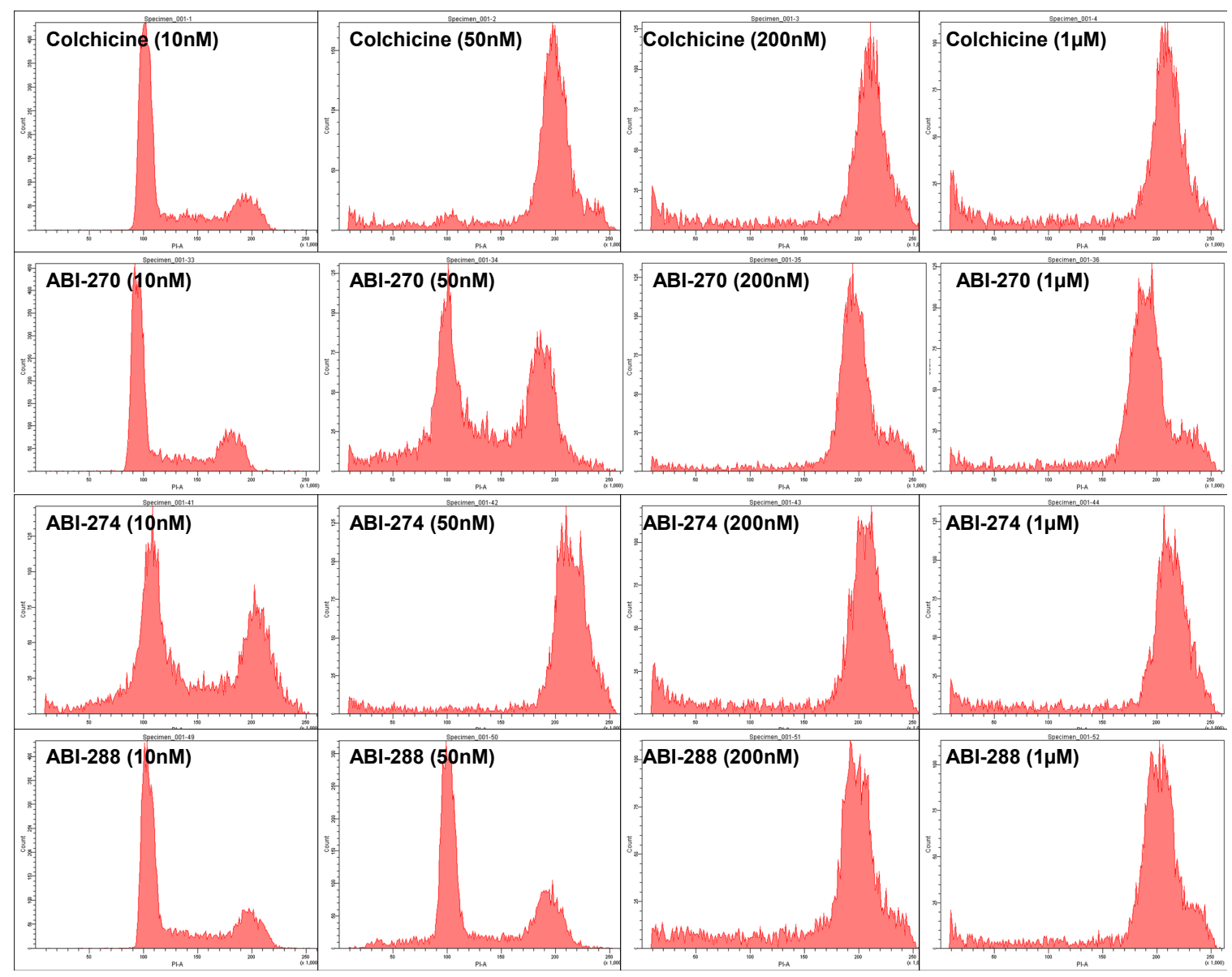

Figure 5-7. Cell cycle analysis of A375 cells after incubation with ABI compounds and colchicine.

Colchicine arrested most cells into G2/M phase starting from $50 \mathrm{nM}$. ABI-270, 274, and 288 also arrested most cells into G2/M starting from 200, 50, and $200 \mathrm{nM}$ respectively. 


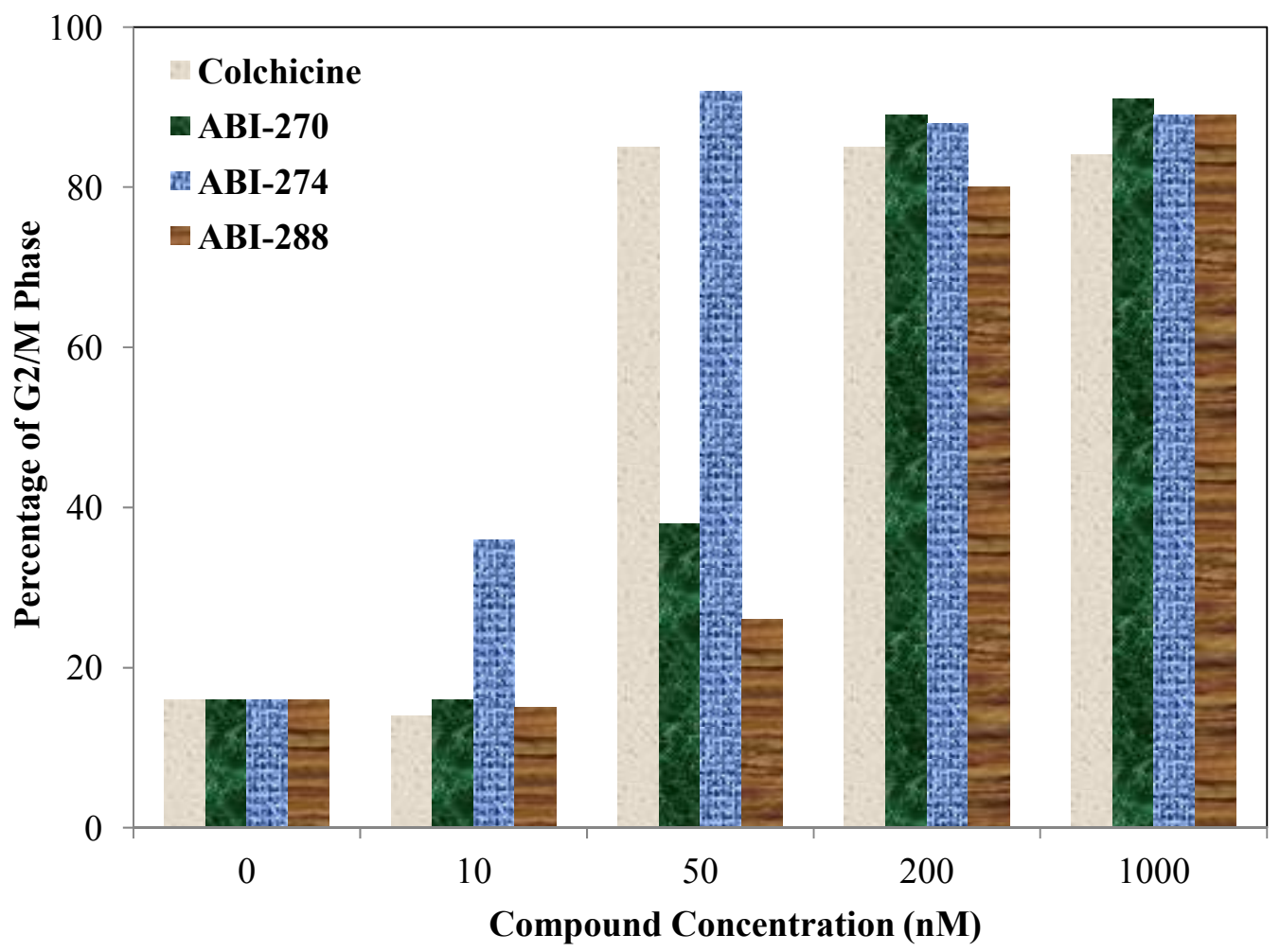

Figure 5-8. Quantified graphic depiction of cell cycle analysis.

ABI-274 showed greater potency than colchicine in arresting cells into G2/M phase. Although ABI-270 and 288 showed lower potency, they also arrested most cells into $\mathrm{G} 2 / \mathrm{M}$ phase at higher concentration. 
increased from $14 \%$ to $85 \%$. ABIs had similar results for $\mathrm{A} 375$ cells, in arresting them in the $\mathrm{G} 2 / \mathrm{M}$ phase in a dose-dependent manner. The potency of the different concentrations in arresting cells in the G2/M phase positively correlated with in vitro activity.

\subsubsection{In Vitro Tubulin Polymerization Assay}

The findings of the cell cycle analysis strongly supported our hypothesis and guided us to test ABIs' direct effect on tubulin polymerization. I conducted an in vitro tubulin polymerization assay by using purified bovine brain tubulin monomer (Figure 5-9). Three tested compounds - ABI-270, -274, and -288 - all showed a strong inhibitory effect on tubulin polymerization. At a concentration of $10 \mu \mathrm{M}$ and 15 min of incubation, the compounds inhibited tubulin polymerization to $17 \%, 3 \%$, and $22 \%$, respectively, compared with control (5\% DMSO was used in controls as a promoter for tubulin polymerization and to compensate DMSO usage in compound testing). This microtubule destabilization effect was similar to that of colchicine and vinblastine but was opposite to that of paclitaxel. The results not only confirmed that ABIs can directly interact with tubulin but also suggested that they may share the same binding site with colchicine or vinblastine. Our next goal was to determine the exact binding site for our compounds.

\subsubsection{Molecular Modeling}

Based on the structure similarity of ABI compounds with colchicine and using molecular modeling techniques, Dr. Li first tested whether our compounds could bind to the colchicine binding site on tubulin $(147,148)$. All three compounds showed excellent docking scores and their binding poses overlaps with the native ligand very well. As an example, the binding mode of ABI-288 (stick model) is shown in Figure 5-10. For comparison, he also displayed the native ligand DAMA-colchicine as a wire model in the $\alpha / \beta$-tubulin heterodimer. The overall structure of ABI-288 and DAMA-colchicine overlapped very well. The $p$-Fluoro phenyl moiety overlaps with the trimethoxylpheny moiety which is interacting with the T7 loop in the $\beta$-subunit. Similarly, the $p$-Chloro phenyl moiety occupies the other side of the pocket where the seven-member ring of the DAMA-colchicine is, with the chlorine atom occupying the pocket where the methoxy moiety interacts. This exciting finding encouraged us to search further for more solid experimental proof to confirm the binding.

\subsubsection{Tubulin Colchicine Site Binding Assay}

After comparison, rapid [3H]colchicine competition-binding scintillation proximity assay (SPA) was chosen to confirm the binding site and to measure binding affinity (131). This method uses SPA technology and requires a close association between a solid-phase scintillant (the beads) and the radioligand for a signal to be emitted and subsequently detected. Biotin tubulin was the reagent that brings the radioligand and the scintillant into close association. When using tritiated colchicine as radioligand, the 


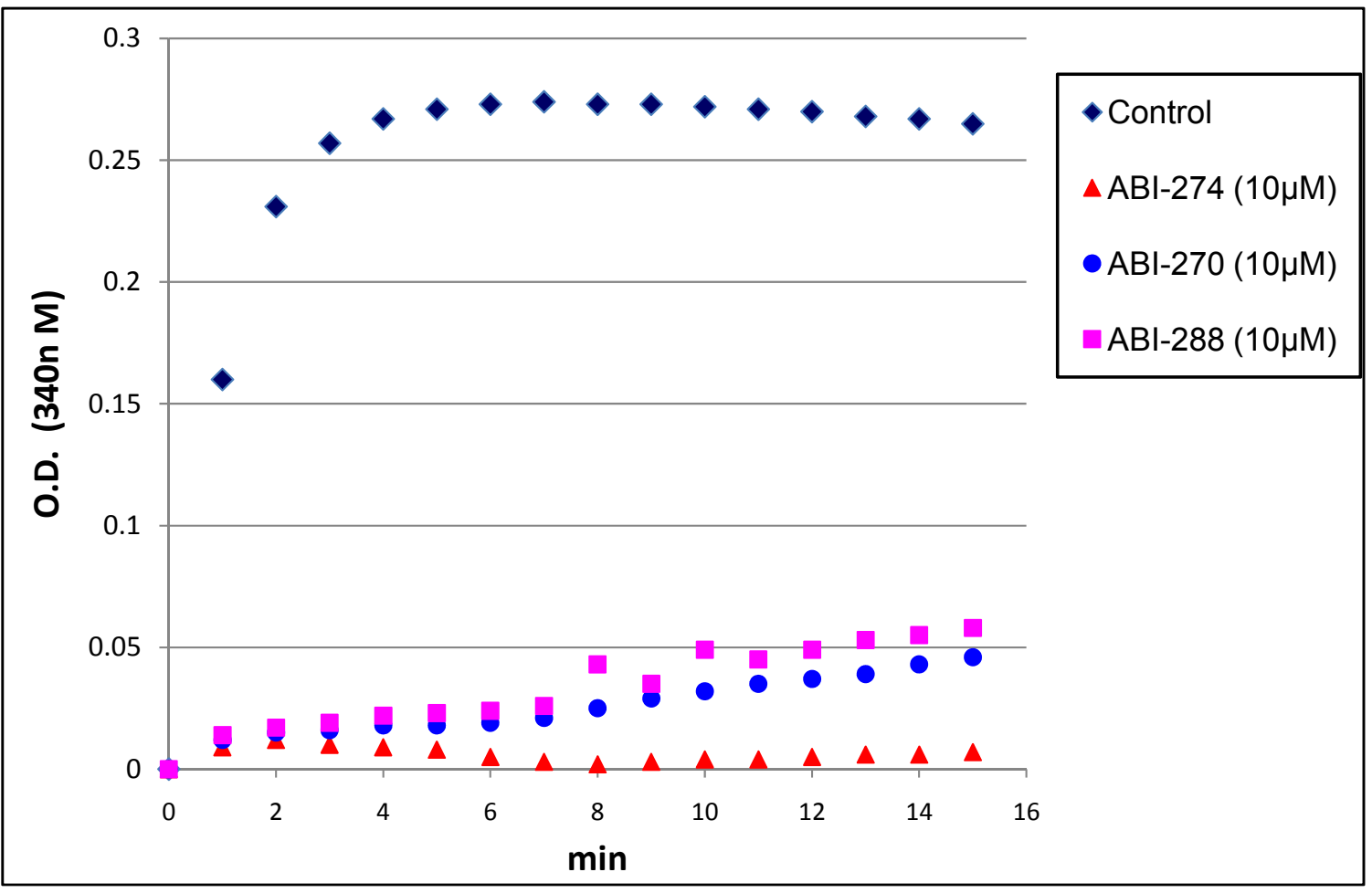

Figure 5-9. Three representative ABI compounds inhibited in vitro tubulin polymerization at a concentration of $10 \mu \mathrm{M}$.

After $15 \mathrm{~min}$ incubation, ABI-274, 288, and 270 inhibited tubulin polymerization to $3 \%$, $17 \%$, and $22 \%$ respectively compared with control (5\% DMSO in general tubulin buffer). This experiment was done by Mr. Chien-Ming Li, Division of Pharmaceutics, College of Pharmacy, the Ohio State University. 


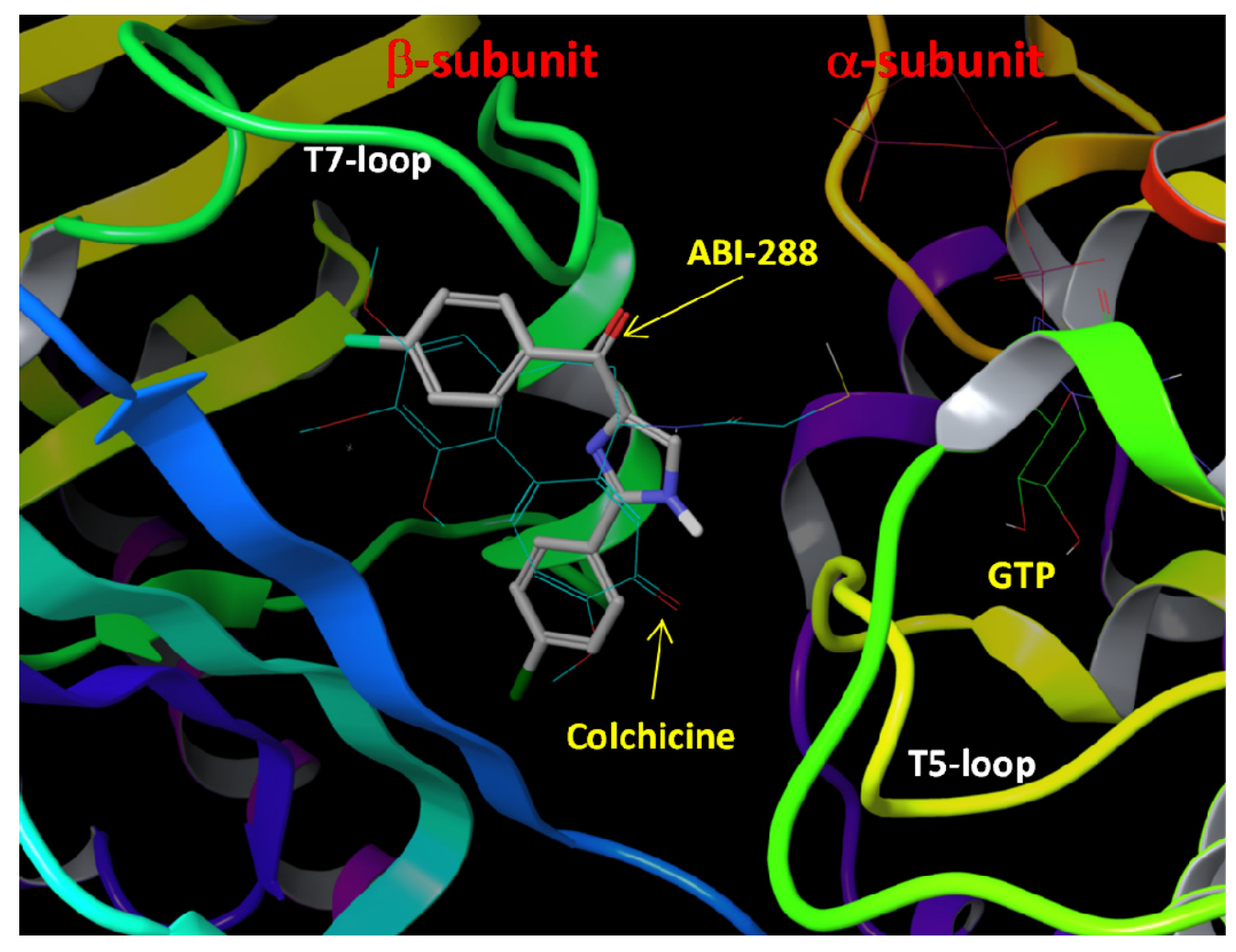

Figure 5-10. Docking of ABI-288 into tubulin crystal structure at the colchicine binding pocket.

Only the related secondary structures in tubulin are shown; ABT-751 is shown as a wire model. The overall structure of ABI-288 and ABT-751 overlapped very well in the binding pocket. The multiple interactions between ABI-288 and tubulin dimer suggested that this compound may have high binding affinity to this pocket. This experiment was done by Dr. Wei Li. 
amplitude of signal was proportional to the number of colchicine binding sites that were occupied by this radioligand. When adding a competitor of radio-labeled colchicine, e.g., cold (unlabeled) colchicine, to the mixture, the signal amplitude decreased proportionately with the concentration of the competitor. This method is very specific for tubulin colchicine site binding competitors.

The binding graph (Figure 5-11) clearly shows that ABIs can competitively bind to the tubulin colchicine binding site. As the concentration of the three tested compounds increased from $0.03 \mu \mathrm{M}$ to $100 \mu \mathrm{M}$, increased tritiated colchicine was competitively stripped away from tubulin and emitted lower SPA counts. The negative control, paclitaxel, gave only a flat line, because theoretically it should not bind to the colchicine binding site on tubulin. Second, ABIs have relatively high binding affinity to the tubulin colchicine binding site (Table 5-6). GraphPad Prism calculated $\mathrm{IC}_{50}$ values for binding showed that ABI-274 has the highest binding affinity among the three tested compounds. Its binding affinity was even higher than that of colchicine. The other two compounds, ABI-270 and ABI-288, showed a little lower affinity to this site than did the natural ligand colchicine, but they were still very close. Third, the binding affinity was positively correlated to in vitro anti-melanoma activity; the higher the binding affinity, the higher the anti-melanoma activity.

According to the Cheng-Prusoff equation, $\mathrm{Ki}=\mathrm{IC}_{50} /(1+\mathrm{D} / \mathrm{Kd})$, where $\mathrm{Ki}$ is the inhibition constant, $\mathrm{D}$ is the concentration of the radioligand, the $\mathrm{Kd}$ is the dissociation constant of the radioligand to tubulin (149). In our experiment, the D value was $0.08 \mu \mathrm{M}$, and the $\mathrm{Kd}$ value was around $1 \mu \mathrm{M}$ according to the literature (131). So the $\mathrm{IC}_{50}$ was very close to the Ki value. Our result was comparable with the Ki value reported in the literature for colchicine binding to tubulin.

\subsubsection{Microtubule Imaging Using Immunofluorescence Microscope}

To obtain visual proof of ABIs interacting with tubulin inside the cells, I examined microtubule arrangement in human melanoma WM-164 cells upon treatment with different compounds (Figure 5-12). The microtubule images clearly showed that all five tested compounds resulted in cytoskeleton rearrangement. There was a significant difference between paclitaxel and the other four compounds (colchicine, ABT-751, ABI270 , and 274). Treatment with paclitaxel resulted in a condensation of microtubules orderly lying around the nuclei compared with controls, consistent with its mechanisms of action for stabilizing microtubules. On the contrary, treatment with colchicine, ABT751, ABI-270, or 274 had similar effects on microtubules and resulted in some degree of microtubule fragmentation, consistent with their common mechanism of action for destabilizing microtubules. These results also confirmed that ABIs shared the same cellular target with colchicine and induced the same cellular effect. 


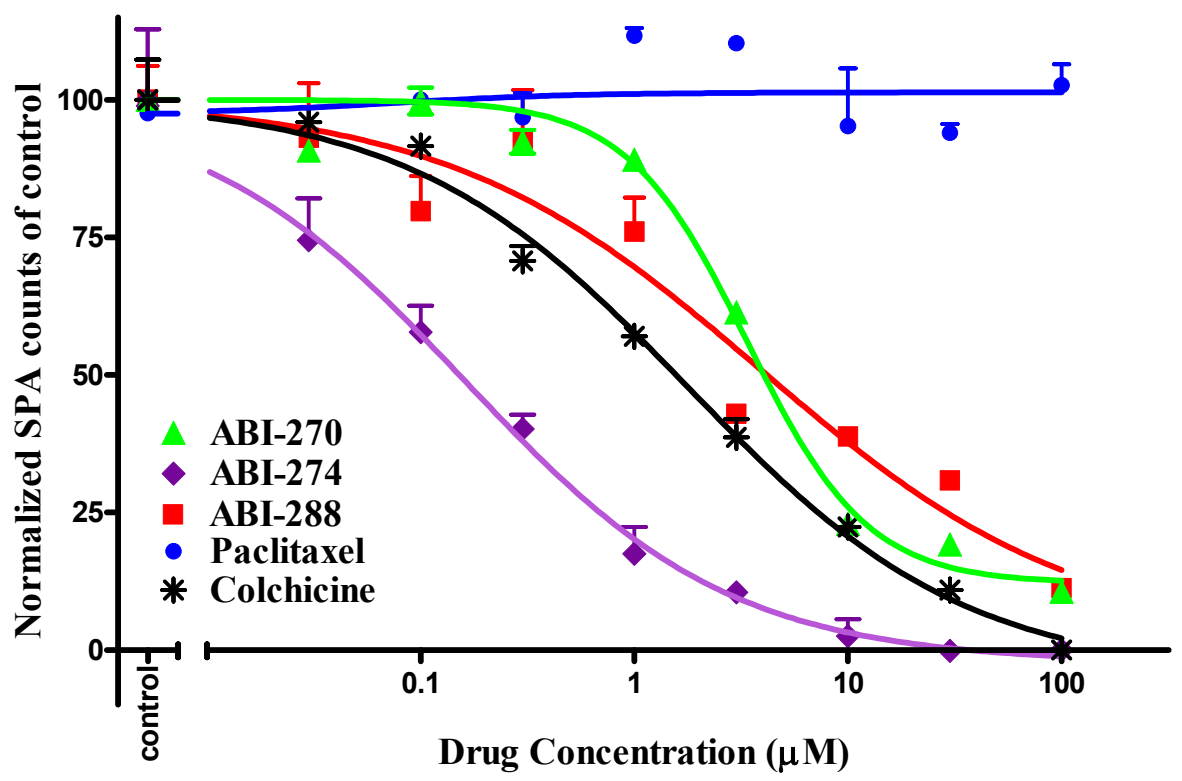

Figure 5-11. $[3 \mathrm{H}]$ colchicine competition-binding assay confirmed ABI compounds competitively bound to tubulin at the colchicine binding site.

As concentration of these compounds increased from $0.03 \mu \mathrm{M}$ to $100 \mu \mathrm{M}$, more and more tritiated colchicine were competitively stripped away from tubulin. Thus gave out lower and lower SPA counts. The negative control paclitaxel just gave out a flat line because it didn't bind to the colchicine binding site on tubulin. Each data point represents an average of three replicates.

Table 5-6. Comparison of tubulin binding affinity and in vitro cytotoxic activity between ABI compounds and known tubulin interacting drugs.

\begin{tabular}{ccc}
\hline \multirow{2}{*}{ Compound name } & \multicolumn{2}{c}{$\mathrm{IC}_{50} \pm \operatorname{SEM}(\mathrm{n}=3)$} \\
\cline { 2 - 3 } & For tubulin binding $(\mu \mathrm{M})$ & On $\mathrm{A} 375(\mathrm{nM})$ \\
\hline ABI-270 & $3 \pm 2$ & $31 \pm 5$ \\
ABI-274 & $200 \pm 100 \mathrm{nM}$ & $9 \pm 2$ \\
ABI-288 & $4 \pm 2$ & $52 \pm 4$ \\
Colchicine & $2 \pm 1$ & $20 \pm 3$ \\
Paclitaxel & N/A & $12 \pm 3$ \\
\hline
\end{tabular}

N/A: not available 

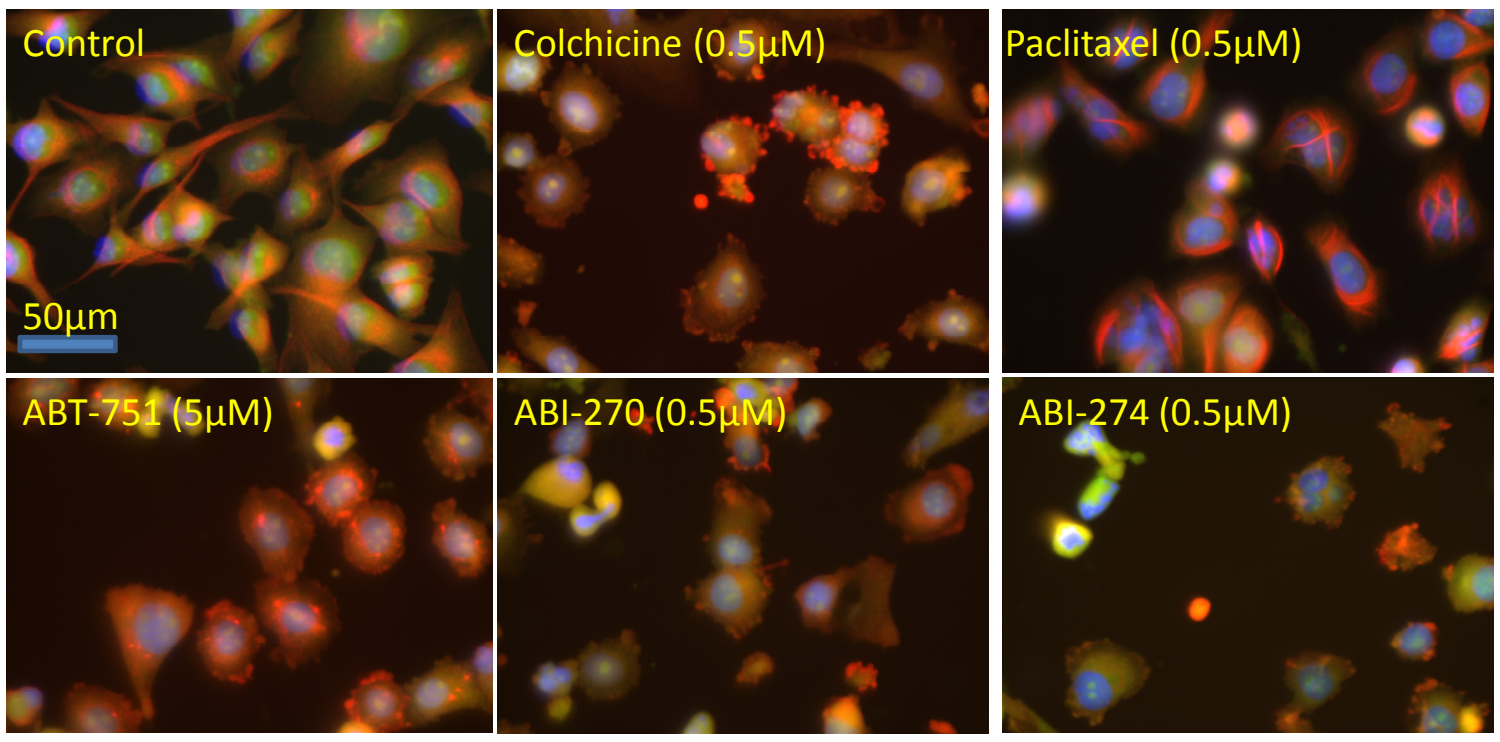

Figure 5-12. Microscopic images of immunofluorescence labeled microtubules inside melanoma cells after compounds treatment.

Treatment with paclitaxel resulted in condensation of microtubules nicely laying around the nuclei compared with control, consistent with its mechanisms of action for stabilizing microtubules. On the contrary, treatment with colchicine, ABT-751, ABI-270 or -274 all had similar effect on microtubules, and resulted in some degree of microtubule fragmentations, consistent with their common mechanism of action for destabilizing microtubules. 


\subsection{Conclusions}

Our team synthesized a set of novel 2-aryl-4-benzoyl-imidazole (ABI) derivatives that showed potent activity in a number of melanoma cell lines, as well as in a xenograft model. The compounds inhibited tubulin polymerization by binding to the colchicine binding site. Structure-activity-relationships were investigated by introducing different substituents into the $\mathrm{A}$ and $\mathrm{C}$ rings. Compared with the earlier SMART compounds, ABI analogs showed two improvements. Firstly, ABI compounds showed much better resistance index (1) compared with colchicine (66), paclitaxel (69), and vinblastine (28). The fact that ABIs can overcome multi-drug resistance suggests a promising future development of more drug-like agents. Secondly, our in vivo study showed that compound ABI-288 at a dose of $25 \mathrm{mg} / \mathrm{kg}$ gave better tumor suppression than that of DTIC at a dose of $60 \mathrm{mg} / \mathrm{kg}$. 


\section{LIST OF REFERENCES}

1. Li G, Satyamoorthy K, Herlyn M. Dynamics of cell interactions and communications during melanoma development. Crit Rev Oral Biol Med 2002;13:62-70.

2. Halaban R. The regulation of normal melanocyte proliferation. Pigment Cell Res 2000;13:4-14.

3. Miller AJ, Mihm MC. Melanoma. N Engl J Med 2006;355:51-65.

4. Gray-Schopfer V, Wellbrock C, Marais R. Melanoma biology and new targeted therapy. Nature 2007;445:851-7.

5. Jemal A, Thomas A, Murray T, et al. Cancer statistics, 2002. Cancer J Clin 2002;52:23-47.

6. Vries E, Bray FI, Coebergh JW, et al. Changing epidemiology of malignant cutaneous melanoma in Europe 1953-1997: rising trends in incidence and mortality but recent stabilizations in western Europe and decreases in Scandinavia. Int J Cancer 2003;107:119-26.

7. Ries L, Melbert D, Krapcho M, et al. SEER cancer statistics review, 1975-2005. Bethesda: National Cancer Institute; 2008.

8. Lucas R. Global burden of disease of solar ultraviolet radiation, environmental burden of disease series. News release. Report No. 13. World Health Organization; 2006.

9. Williams DA, Lemke TL, editors. Foye's principles of medicinal chemistry. $6^{\text {th }}$ ed. Philadelphia: Lippincott Williams \& Wilkins; 2007.

10. Falkson CI, Ibrahim J, Kirkwood JM, et al. Phase III trial of dacarbazine versus dacarbazine with interferon alpha- $2 \mathrm{~b}$ versus dacarbazine with tamoxifen versus dacarbazine with interferon alpha- $2 \mathrm{~b}$ and tamoxifen in patients with metastatic malignant melanoma: an eastern cooperative oncology group study. J Clin Oncol 1998;16:1743-51.

11. Chapman PB, Einhorn LH, Meyers ML, et al. Phase III multicenter randomized trial of the dartmouth regimen versus dacarbazine in patients with metastatic melanoma. J Clin Oncol 1999;17:2745-51.

12. Eggermont AM, Kirkwood JM. Re-evaluating the role of dacarbazine in metastatic melanoma: what have we learned in 30 years? Eur J Cancer 2004;40:1825-36. 
13. Smith FO, Downey SG, Klapper JA, et al. Treatment of metastatic melanoma using interleukin-2 alone or in conjunction with vaccines. Clin Cancer Res 2008;14:5610-8.

14. Riker AI, Radfar S, Liu S, et al. Immunotherapy of melanoma: a critical review of current concepts and future strategies. Expert Opin Biol Ther 2007;7:345-58.

15. Quan W, Brick W, Vinogradov M, et al. Repeated cycles with 72-hour continuous infusion interleukin-2 in kidney cancer and melanoma. Cancer Biother Radiopharm 2004;19:350-4.

16. Kiessling R, Wasserman $\mathrm{K}$, Horiguchi $\mathrm{S}$, et al. Tumor-induced immune dysfunction. Cancer Immunol Immunother 1999;48:353-62.

17. Jacinto FV, Esteller M. MGMT hypermethylation: a prognostic foe, a predictive friend. DNA Repa (Amst) 2007;6:1155-60.

18. Newlands ES, Blackledge GR, Slack JA, et al. Phase I trial of temozolomide (CCRG 81045: M\&B 39831: NSC 362856). Br J Cancer 1992;65:287-91.

19. Middleton MR, Grob JJ, Aaronson N, et al. Randomized phase III study of temozolomide versus dacarbazine in the treatment of patients with advanced metastatic malignant melanoma. J Clin Oncol 2000;18:158-66.

20. Strumberg D, Richly H, Hilger RA, et al. Phase I clinical and pharmacokinetic study of the novel raf kinase and vascular endothelial growth factor receptor inhibitor BAY 43-9006 in patients with advanced refractory solid tumors. J Clin Oncol 2005;23:965-72.

21. Ahmad T, Pyle L, James M, et al. BAY 43-9006 in patients with advanced melanoma: the royal marsden experience. J Clin Oncol 2004;22:7506.

22. Eisen T, Ahmad T, Flaherty KT, et al. Sorafenib in advanced melanoma: a phase II randomised discontinuation trial analysis. Br J Cancer 2006;95:581-6.

23. Flaherty KT, Brose BM, Schucter L, et al. Phase I/II trial of BAY 43-9006, carboplatin (C) and paclitaxel (P) demonstrates preliminary antitumor activity in the expansion cohort of patients with metastatic melanoma. J Clin Oncol 2004;22:7507.

24. Herlyn M. Recent development in melanoma research (review on literature). Soci Mela Res News 2007;4:3-5.

25. Atkins MB. The treatment of metastatic melanoma with chemotherapy and biologics. Curr Opin Oncol 1997;9:205-13. 
26. Evans LM, Casper ES, Rosenbluth R. Phase II trial of carboplatin in advanced malignant melanoma. Cancer Treat Rep 1987;71:171-2.

27. Gogas HJ, Kirkwood JM, Sondak VK. Chemotherapy for metastatic melanoma: time for a change? Cancer 2007;109:455-64.

28. Boaziz C, Breau JL, Morere JF, et al. Brain metastases of malignant melanomas. Bull Cancer 1991;78:347-53.

29. Quagliana JM, Stephens RL, Baker LH, et al. Vindesine in patients with metastatic malignant melanoma: a southwest oncology group study. J Clin Oncol 1984;2:316-9.

30. Bedikian AY, Weiss GR, Legha SS, et al. Phase II trial of docetaxel in patients with advanced cutaneous malignant melanoma previously untreated with chemotherapy. J Clin Oncol 1995;13:2895-9.

31. Avril MF, Bonneterre J, Delaunay M, et al. Combination chemotherapy of dacarbazine and fotemustine in disseminated malignant melanoma, experience of the french study group. Cancer Chemother Pharmacol 1990;27:81-4.

32. Fletcher WS, Green S, Fletcher JR, et al. Evaluation of cisplatin and DTIC combination chemotherapy in disseminated melanoma: a southwest oncology group study. Am J Clin Oncol 1988;11:589-93.

33. Vorobiof DA, Sarli R, Falkson G. Combination chemotherapy with dacarbazine and vindesine in the treatment of metastatic malignant melanoma. Cancer Treat Rep 1986;70:927-8.

34. Legha SS, Ring S, Papadopoulos N, et al. A prospective evaluation of a tripledrug regimen containing cisplatin, vinblastine, and dacarbazine (CVD) for metastatic melanoma. Cancer 1989;64:2024-9.

35. Prete SA, Maurer LH, O'Donnell J, et al. Combination chemotherapy with cisplatin, carmustine, dacarbazine, and tamoxifen in metastatic melanoma. Cancer Treat Rep 1984;68:1403-5.

36. Flaherty LE, Liu PY, Unger J, et al. Comparison of patient characteristics and outcome between a single-institution phase II trial and a cooperative-group phase II trial with identical eligibility in metastatic melanoma. Am J Clin Oncol 1997;20:600-4.

37. Liu YJ. IPC: professional type 1 interferon-producing cells and plasmacytoid dendritic cell precursors. Annu Rev Immunol 2005;23:275-306. 
38. Moschos SJ, Edington HD, Land SR, et al. Neoadjuvant treatment of regional stage IIIB melanoma with high-dose interferon alfa- $2 \mathrm{~b}$ induces objective tumor regression in association with modulation of tumor infiltrating host cellular immune responses. J Clin Oncol 2006;24:3164-71.

39. Kirkwood JM, Strawderman MH, Ernstoff MS, et al. Interferon alfa-2b adjuvant therapy of high-risk resected cutaneous melanoma: the eastern cooperative oncology group trial EST 1684. J Clin Oncol 1996;14:7-17.

40. Kirkwood JM, Ibrahim J, Lawson DH, et al. High-dose interferon alfa-2b does not diminish antibody response to GM2 vaccination in patients with resected melanoma: results of the multicenter eastern cooperative oncology group phase II trial E2696. J Clin Oncol 2001;19:1430-6.

41. Gupta A, Kirkwood JM. Melanoma. Kaufman, HL, Wadler S, Antman K, editors. Molecular targeting in oncology. Totowa: Humana Press; 2008.

42. Sangfelt O, Erickson S, Castro J, et al. Induction of apoptosis and inhibition of cell growth are independent responses to interferon-alpha in hematopoietic cell lines. Cell Growth Differ 1997;8:343-52.

43. Kirkwood JM, Bender C, Agarwala S, et al. Mechanisms and management of toxicities associated with high-dose interferon alfa-2b therapy. J Clin Oncol 2002;20:3703-18.

44. Weber J. Review: anti-CTLA-4 antibody ipilimumab: case studies of clinical response and immune-related adverse events. Oncologist 2007;12:864-72.

45. Reuben JM, Lee BN, Li C, et al. Biologic and immunomodulatory events after CTLA-4 blockade with ticilimumab in patients with advanced malignant melanoma. Cancer 2006;106:2437-44.

46. Johnston RL, Lutzky J, Chodhry A, et al. Cytotoxic T-lymphocyte-associated antigen 4 antibody-induced colitis and its management with infliximab. Dig Dis Sci 2009;54:2538-40.

47. Mitjans F, Meyer T, Fittschen C, et al. In vivo therapy of malignant melanoma by means of antagonists of alphav integrins. Int J Cancer 2000;87:716-23.

48. Hersey P, Sosman J, O'Day S, et al. A phase II, randomized, open-label study evaluating the antitumor activity of MEDI-522, a humanized monoclonal antibody directed against the human metastatic melanoma (MM). Proc Am Soc Clin Oncol 2005;23:711. 
49. Hersey P, Sosman J, O'Day S, et al. A randomized phase 2 study of etaracizumab, a monoclonal antibody against integrin alpha(v)beta(3), +/- dacarbazine in patients with stage IV metastatic melanoma. Cancer 2010;116:1526-34.

50. Ward S, Casey D, Labarthe MC, et al. Immunotherapeutic potential of whole tumour cells. Cancer Immunol Immunother 2002;51:351-7.

51. Hsueh EC, Morton DL. Antigen-based immunotherapy of melanoma: Canvaxin therapeutic polyvalent cancer vaccine. Semin Cancer Biol 2003;13:401-7.

52. Market Wire. CancerVax announces results of phase 3 clinical trials of Canvaxin(TM) in patients with stage III and stage IV melanoma. http://ajaxworld.com/node/198642. Accessed February 12, 2010.

53. Sondak VK, Liu PY, Tuthill RJ, et al. Adjuvant immunotherapy of resected, intermediate-thickness, node-negative melanoma with an allogeneic tumor vaccine: overall results of a randomized trial of the Southwest Oncology Group. J Clin Oncol 2002;20:2058-66.

54. News Feature. Rethinking therapeutic cancer vaccines. Nat Rev Drug Discov 2009;8:685-6.

55. Berd D, Sato T, Maguire HC, et al. Immunopharmacologic analysis of an autologous, hapten-modified human melanoma vaccine. J Clin Oncol 2004;22:403-15.

56. Avax Technologies. Harnessing the patient's immune system for the treatment of cancer. http://www.avax-tech.com/about/pdfs/avaxfactsheet.pdf. Accessed February 13, 2010.

57. Riker AI, Panelli MC, Kammula US, et al. Development and characterization of melanoma cell lines established by fine-needle aspiration biopsy: advances in the monitoring of patients with metastatic melanoma. Cancer Detect Prev 1999;23:387-96.

58. Rosenberg SA, Yang JC, Schwartzentruber DJ, et al. Immunologic and therapeutic evaluation of a synthetic peptide vaccine for the treatment of patients with metastatic melanoma. Nat Med 1998;4:321-7.

59. Pipeline Review. Pipeline insight: therapeutic cancer vaccines - prospect of first approval set to reinvigorate interest from major companies. http://www.the-infoshop.com/report/dc105049-cancer-vaccine.html. Accessed February 13, 2010. 
60. Kruit W, Suciu S, Dreno B, et al. Immunization with recombinant MAGE-A3 protein combined with adjuvant systems AS15 or AS02B in patients with unresectable and progressive metastatic cutaneous melanoma: a randomized openlabel phase II study of the EORTC melanoma group. J Clin Oncol 2008;26:9065.

61. Rosenberg SA, Yang JC, Restifo NP. Cancer immunotherapy: moving beyond current vaccines. Nat Med 2004;10:909-15.

62. Slingluff CL, Petroni GR, Yamshchikov GV, et al. Clinical and immunologic results of a randomized phase II trial of vaccination using four melanoma peptides either administered in granulocyte-macrophage colony-stimulating factor in adjuvant or pulsed on dendritic cells. J Clin Oncol 2003;21:4016-26.

63. Albert ML, Sauter B, Bhardwaj N. Dendritic cells acquire antigen from apoptotic cells and induce class I-restricted CTLs. Nature 1998;392:86-9.

64. Ridgway D. The first 1000 dendritic cell vaccinees. Cancer Invest 2003;21:87386.

65. Nestle FO, Alijagic S, Gilliet M, et al. Vaccination of melanoma patients with peptide- or tumor lysate-pulsed dendritic cells. Nat Med 1998;4:328-32.

66. Schadendorf NF, Broecker EB, Enk A, et al. Dacarbacine (DTIC) versus vaccination with autologous peptide-pulsed dendritic cells (DC) as first-line treatment of patients with metastatic melanoma: results of a prospectiverandomized phase III study. J Clin Oncol 2004;22:7508.

67. Rosenberg SA, Dudley ME. Cancer regression in patients with metastatic melanoma after the transfer of autologous antitumor lymphocytes. Proc Natl Acad Sci U S A 2004;101 Suppl 2:14639-45.

68. Sotomayor MG, Yu H, Antonia S, et al. Advances in gene therapy for malignant melanoma. Cancer Contr 2002;9:39-48.

69. Piechocki MP, Pilon SA, Wei WZ. Complementary antitumor immunity induced by plasmid DNA encoding secreted and cytoplasmic human ErbB-2. J Immunol 2001;167:3367-74.

70. Ulmer JB, Donnelly JJ, Parker SE, et al. Heterologous protection against influenza by injection of DNA encoding a viral protein. Science 1993;259:1745-9.

71. Trisciuoglio D, Desideri M, Ciuffreda L, et al. Bcl-2 overexpression in melanoma cells increases tumor progression-associated properties and in vivo tumor growth. J Cell Physiol 2005;205:414-21. 
72. Jansen B, Wacheck V, Heere-Ress E, et al. Chemosensitisation of malignant melanoma by BCL2 antisense therapy. Lancet 2000;356:1728-33.

73. Millward MJ, Conry RM, Gore ME, et al. Randomized multinational phase 3 trial of dacarbazine (DTIC) with or without Bcl-2 antisense (oblimersen sodium) in patients (pts) with advanced malignant melanoma (MM): analysis of long-term survival. J Clin Oncol 2004;22:7505.

74. Medical News Today. FDA advisory committee reviews Genasense(reg) for use in advanced melanoma. http://www.medicalnewstoday.com/articles/7929.php. Accessed February 14, 2010.

75. Vical Inc. Vical completes enrollment in Allovectin-7(r) phase 3 trial for metastatic melanoma. http://ir.vical.com/releasedetail.cfm?ReleaseID=441001. Accessed February 14, 2010.

76. Richards $\mathrm{AB}$, Gonzalez R, Atkins $\mathrm{MB}$, et al. High-dose Allovectin-7 in patients with advanced metastatic melanoma: final phase 2 data and design of phase 3 registration trial. J Clin Oncol 2005;23:7543.

77. Senzer NN, Kaufman H, Amatruda T, et al. Updated results of a phase II clinical trial with a second generation, enhanced potency, immune-enhanced, oncolytic herpesvirus in unresectable metastatic melanoma. http://www.biovex.com/downloads/ASCO_melanoma_poster_2009.pdf. Accessed February 14, 2010.

78. BioVex Inc. Biovex commences OncoVEX (GM-CSF) phase 3 trial in metastatic melanoma. http://www.biovex.com/04_14_09.htm. Accessed February 14, 2010.

79. Dothager RS, Putt KS, Allen BJ, et al. Synthesis and identification of small molecules that potently induce apoptosis in melanoma cells through G1 cell cycle arrest. J Am Chem Soc 2005;127:8686-96.

80. Fernandez Y, Verhaegen M, Miller TP, et al. Differential regulation of noxa in normal melanocytes and melanoma cells by proteasome inhibition: therapeutic implications. Cancer Res 2005;65:6294-304.

81. Smalley KS, Herlyn M. Targeting intracellular signaling pathways as a novel strategy in melanoma therapeutics. Ann N Y Acad Sci 2005;1059:16-25.

82. Gududuru V, Hurh E, Dalton JT, et al. Discovery of 2-arylthiazolidine-4carboxylic acid amides as a new class of cytotoxic agents for prostate cancer. J Med Chem 2005;48:2584-8.

83. Li W, Lu Y, Wang Z, et al. Synthesis and antiproliferative activity of thiazolidine analogs for melanoma. Bioorg Med Chem Lett 2007;17:4113-7. 
84. Chen J, Wang Z, Lu Y, et al. Synthesis and antiproliferative activity of imidazole and imidazoline analogs for melanoma. Bioorg Med Chem Lett 2008;18:3183-7.

85. Li W, Wang Z, Gududuru V, et al. Structure-activity relationship studies of arylthiazolidine amides as selective cytotoxic agents for melanoma. Anticancer Res 2007;27:883-8.

86. Gududuru V, Zeng K, Tsukahara R, et al. Identification of Darmstoff analogs as selective agonists and antagonists of lysophosphatidic acid receptors. Bioorg Med Chem Lett 2006;16:451-6.

87. Gududuru V, Hurh E, Sullivan J, et al. SAR studies of 2-arylthiazolidine-4carboxylic acid amides: a novel class of cytotoxic agents for prostate cancer. Bioorg Med Chem Lett 2005;15:4010-3.

88. Bankston D, Dumas J, Natero R, et al. A scaleable synthesis of BAY 43-9006: a potent raf kinase inhibitor for the treatment of cancer. Org Proc Res Dev 2002;6:777-81.

89. Slominski A, Ermak G, Wortsman J. Modification of melanogenesis in cultured human melanoma cells. In Vitro Cell Dev Biol Anim 1999;35:564-5.

90. Rubinstein LV, Shoemaker RH, Paull KD, et al. Comparison of in vitro anticancer-drug-screening data generated with a tetrazolium assay versus a protein assay against a diverse panel of human tumor cell lines. J Natl Cancer Inst 1990;82:1113-8.

91. Slominski A, Pisarchik A, Zbytek B, et al. Functional activity of serotoninergic and melatoninergic systems expressed in the skin. J Cell Physiol 2003;196:144-53.

92. Ormerod MG, editor. Flow cytometry, a practical approach. $3^{\text {rd }}$ ed. Oxford: Oxford University Press; 2000.

93. Zmijewski MA, Li W, Zjawiony JK, et al. Photo-conversion of two epimers (20R and 20S) of pregna-5,7-diene-3beta, 17alpha, 20-triol and their bioactivity in melanoma cells. Steroids 2009;74:218-28.

94. Guo W, Wu S, Liu J, et al. Identification of a small molecule with synthetic lethality for K-ras and protein kinase C iota. Cancer Res 2008;68:7403-8.

95. Chen J, Smith M, Kolinsky K, et al. Antitumor activity of HER1/EGFR tyrosine kinase inhibitor erlotinib, alone and in combination with CPT-11 (irinotecan) in human colorectal cancer xenograft models. Cancer Chemother Pharmacol 2007;59:651-9. 
96. Daidone G, Maggio B, Raffa D, et al. Synthesis and in vitro antileukemic activity of new 4-triazenopyrazole derivatives. Farmaco 2004;59:413-7.

97. Wilhelm S, Carter C, Lynch M, et al. Discovery and development of Sorafenib: a multikinase inhibitor for treating cancer. Nat Rev Drug Discov 2006;5:835-44.

98. McFadyen MC, Melvin WT, Murray GI. Cytochrome P450 enzymes: novel options for cancer therapeutics. Mol Cancer Ther 2004;3:363-71.

99. Rooseboom M, Commandeur JN, Vermeulen N. Enzyme-catalyzed activation of anticancer prodrugs. Pharmacol Rev 2004;56:53-102.

100. Lu Y, Li CM, Wang Z, et al. Discovery of 4-substituted methoxybenzoyl-arylthiazole as novel anticancer agents: synthesis, biological evaluation, and structure-activity relationships. J Med Chem 2009;52:1701-11.

101. Brugarolas A, Gosalvez M. Treatment of cancer by an inducer of reverse transformation. Lancet 1980;1:68-70.

102. Liu J, Qu S, Li B, et al. Synthesis and antitumor activity of timonacic derivatives. Yiyao Gongye 1988;19:203-5.

103. Williams DA, Lemke TL, editors. Foye's principles of medicinal chemistry. $5^{\text {th }}$ ed. Philadelphia: Lippincott Williams \& Wilkins; 2002.

104. Meyskens FL, Moon TE, Dana B, et al. Quantitation of drug sensitivity by human metastatic melanoma colony-forming units. Br J Cancer 1981;44:787-97.

105. Von Hoff DD, Casper J, Bradley E, et al. Association between human tumor colony-forming assay results and response of an individual patient's tumor to chemotherapy. Am J Med 1981;70:1027-41.

106. Zmijewski MA, Li W, Zjawiony JK, et al. Synthesis and photo-conversion of androsta- and pregna-5,7-dienes to vitamin D3-like derivatives. Photochem Photobiol Sci 2008;7:1570-6.

107. Zbytek B, Janjetovic Z, Tuckey RC, et al. 20-Hydroxyvitamin D3, a product of vitamin D3 hydroxylation by cytochrome P450scc, stimulates keratinocyte differentiation. J Invest Dermatol 2008;128:2271-80.

108. Constantinides PP, Marcello J, Chiossone DC, et al. Enhanced intestinal absorption of an RGD peptide from water-in-oil microemulsions of different composition and particle size. J Contr Rele 1995;34:109-16.

109. Povlsen CO, Jacobsen GK. Chemotherapy of a human malignant melanoma transplanted in the nude mouse. Cancer Res 1975;35:2790-6. 
110. Lu Y, Li CM, Wang Z, et al. Discovery of 4-substituted methoxybenzoyl-arylthiazole as novel anticancer agents: synthesis, biological evaluation, and structure-activity relationships. J Med Chem 2009;52:1701-11.

111. Zhou D, Alelyunas Y, Liu R. Scores of extended connectivity fingerprint as descriptors in QSPR study of melting point and aqueous solubility. J Chem Inf Model 2008;48:981-7.

112. Yoon S, Smellie A, Hartsough D, Filikov A. Surrogate docking: structure-based virtual screening at high throughput speed. J Comput Aided Mol Des 2005; 19:483-97.

113. Hert J, Willett P, Wilton DJ, et al. Comparison of topological descriptors for similarity-based virtual screening using multiple bioactive reference structures. Org Biomol Chem 2004;2:3256-66.

114. Shellman YG, Ribble D, Miller L, et al. Lovastatin-induced apoptosis in human melanoma cell lines. Melanoma Res 2005;15:83-9.

115. Li W, Lu Y, Wang Z, et al. Synthesis and antiproliferative activity of thiazolidine analogs for melanoma. Bioorg Med Chem Lett 2007;17:4113-7.

116. Longui CA, Santos MC, Formiga CB, et al. Antiproliferative and apoptotic potencies of glucocorticoids: nonconcordance with their antiinflammatory and immunosuppressive properties. Arq Bras Endocrinol Metabol 2005;49:378-83.

117. Hadfield JA, Ducki S, Hirst N, et al. Tubulin and microtubules as targets for anticancer drugs. Prog Cell Cycle Res 2003;5:309-25.

118. Honore S, Pasquier E, Braguer D. Understanding microtubule dynamics for improved cancer therapy. Cell Mol Life Sci 2005;62:3039-56.

119. Jordan A, Hadfield JA, Lawrence NJ, et al. Tubulin as a target for anticancer drugs: agents which interact with the mitotic spindle. Med Res Rev 1998;18:25996.

120. Jordan MA, Kamath K. How do microtubule-targeted drugs work? Curr Cancer Drug Targets 2007;7:730-42.

121. Uppal SO, Li Y, Wendt E, et al. Pattern analysis of microtubule-polymerizing and depolymerizing agent combinations as cancer chemotherapies. Int J Oncol 2007;31:1281-91.

122. Photiou A, Shah $\mathrm{P}$, Leong LK, et al. In vitro synergy of paclitaxel (Taxol) and vinorelbine (navelbine) against human melanoma cell lines. Eur J Cancer 1997;33:463-70. 
123. Germann UA. P-glycoprotein--a mediator of multi-drug resistance in tumour cells. Eur J Cancer 1996;32A:927-44.

124. Dumontet C, Sikic BI. Mechanisms of action of and resistance to antitubulin agents: microtubule dynamics, drug transport, and cell death. J Clin Oncol 1999; 17:1061-70.

125. Mohan R, Banerjee M, Ray A, et al. Antimitotic sulfonamides inhibit microtubule assembly dynamics and cancer cell proliferation. Biochemistry 2006;45:5440-9.

126. Chaplin DJ, Horsman MR, Siemann DW. Current development status of smallmolecule vascular disrupting agents. Curr Opin Investig Drugs 2006;7:522-8.

127. Sampath D, Discafani CM, Loganzo F, et al. MAC-321, a novel taxane with greater efficacy than paclitaxel and docetaxel in vitro and in vivo. Mol Cancer Ther 2003;2:873-84.

128. Liberatore AM, Coulomb H, Pons D, et al. IRC-083927 is a new tubulin binder that inhibits growth of human tumor cells resistant to standard tubulin-binding agents. Mol Cancer Ther 2008;7:2426-34.

129. Li W, Shao Y, Hu L, et al. BM6, a new semi-synthetic vinca alkaloid, exhibits its potent in vivo anti-tumor activities via its high binding affinity for tubulin and improved pharmacokinetic profiles. Cancer Biol Ther 2007;6:787-94.

130. Liou JP, Hsu KS, Kuo CC, et al. A novel oral indoline-sulfonamide agent, N-[1(4-methoxybenzenesulfonyl)-2,3-dihydro-1H-indol-7-yl]-isonicotinamide (J30), exhibits potent activity against human cancer cells in vitro and in vivo through the disruption of microtubule. J Pharmacol Exp Ther 2007;323:398-405.

131. Tahir SK, Kovar P, Rosenberg SH. Rapid colchicine competition-binding scintillation proximity assay using biotin-labeled tubulin. Biotechniques 2000;29:156-60.

132. Gursoy RN, Benita S. Self-emulsifying drug delivery systems (SEDDS) for improved oral delivery of lipophilic drugs. Biomed Pharmacother 2004;58:173-82.

133. Amir E, Rozen S. Easy access to the family of thiazole N-oxides using HOFx $\mathrm{CH}_{3} \mathrm{CN}$. Chem Commun (Camb) 2006:2262-4.

134. Gourdeau H, Leblond L, Hamelin B, et al. Species differences in troxacitabine pharmacokinetics and pharmacodynamics: implications for clinical development. Clin Cancer Res 2004;10:7692-702.

135. Bhattacharyya B, Panda D, Gupta S, et al. Anti-mitotic activity of colchicine and the structural basis for its interaction with tubulin. Medi Res Rev 2008;28:155-83. 
136. Romagnoli R, Baraldi PG, Sarkar T, et al. Synthesis and biological evaluation of 1-methyl-2-(3',4',5'-trimethoxybenzoyl)-3-aminoindoles as a new class of antimitotic agents and tubulin inhibitors. J Med Chem 2008;51:1464-8.

137. Romagnoli R, Baraldi PG, Carrion MD, et al. Synthesis and biological evaluation of 2- and 3-aminobenzo[b]thiophene derivatives as antimitotic agents and inhibitors of tubulin polymerization. J Med Chem 2007;50:2273-7.

138. Lavelle F, Gueritte-Voegelein F, Guenard D. Taxotere: from yew's needles to clinical practice. Bull Cancer 1993;80:326-38.

139. Nelson RL. The comparative clinical pharmacology and pharmacokinetics of vindesine, vincristine, and vinblastine in human patients with cancer. Med Pediatr Oncol 1982;10:115-27.

140. Beerepoot LV, Radema SA, Witteveen EO, et al. Phase I clinical evaluation of weekly administration of the novel vascular-targeting agent, ZD6126, in patients with solid tumors. J Clin Oncol 2006;24:1491-8.

141. Bross PF, Kane R, Farrell AT, et al. Approval summary for bortezomib for injection in the treatment of multiple myeloma. Clin Cancer Res 2004;10:3954-64.

142. Galmarini CM. ABT-751 (Abbott). Curr Opin Investig Drugs 2005;6:623-30.

143. Bellina F, Cauteruccio S, Monti S, et al. Novel imidazole-based combretastatin A-4 analogues: evaluation of their in vitro antitumor activity and molecular modeling study of their binding to the colchicine site of tubulin. Bioorg Med Chem Lett 2006;16:5757-62.

144. Wang L, Woods KW, Li Q, et al. Potent, orally active heterocycle-based combretastatin a-4 analogues: synthesis, structure-activity relationship, pharmacokinetics, and in vivo antitumor activity evaluation. J Med Chem 2002;45:1697-711.

145. Gottesman MM, Fojo T, Bates SE. Multi-drug resistance in cancer: role of ATPdependent transporters. Nat Rev Cancer 2002;2:48-58.

146. Salmon SE. Human tumor clonogenic assays: growth conditions and applications. Cancer Genet Cytogenet 1986;19:21-8.

147. Gigant B, Wang C, Ravelli RB, et al. Structural basis for the regulation of tubulin by vinblastine. Nature 2005;435:519-22.

148. Ravelli RB, Gigant B, Curmi PA, et al. Insight into tubulin regulation from a complex with colchicine and a stathmin-like domain. Nature 2004;428:198-202. 
149. Cheng Y, Prusoff WH. Relationship between the inhibition constant (K1) and the concentration of inhibitor which causes 50 per cent inhibition (I50) of an enzymatic reaction. Biochem Pharmacol 1973;22:3099-108. 


\section{APPENDIX \\ Tubulin Ligand (Colchicine) Competition Assay Protocol}

\section{The basic approach to the assay is as follows}

1. Set-up scintillation plate reader and warm plate in reader to $37^{\circ} \mathrm{C}$.

2. Prepare compounds to $20 \mathrm{x}$ strength $(1 \mathrm{mM})$ in G-PEM buffer plus $10 \%$ DMSO.

3. Prepare tritiated colchicine. Assay life $24 \mathrm{~h}$.

4. $\quad$ Prepare biotin tubulin on ice. Assay life $4 \mathrm{~h}$.

5. Prepare streptavidin beads. Assay life $24 \mathrm{~h}$.

6. Prepare bead/tubulin mixture. Assay life $24 \mathrm{~h}$.

7. Pipette $10 \mu \mathrm{L}$ of compound into the wells.

8. $\quad$ Pipette $10 \mu \mathrm{L}$ of tritiated colchicine into the wells.

9. Pipette $180 \mu \mathrm{L}$ of bead/tubulin into the wells.

10. Incubate for $45 \mathrm{~min}$ at $37^{\circ} \mathrm{C}$.

11. Read plate.

12. Extract the raw data and calculate percent inhibition.

\section{Preparation of compounds}

This step is performed before the tubulin is rehydrated because if there is an unforeseen error or precipitation occurs, then the experiment can be aborted before rehydrating tubulin. Prepare compound stocks at 20x final concentration (usually $1 \mathrm{mM}$ ) in $50 \%$ DMSO, 50\% Ethanol or G-PEM plus one of these solvents from 5 50\% at room temperature. The final organic solvent concentration should not exceed $2.5 \%(\mathrm{v} / \mathrm{v})$. Solubilizing agents such as SDS or Triton X100 can be used up to $0.1 \%$ and $1.0 \%$ respectively. The concentration of compounds may vary depending on the nature of the screen, $50 \mu \mathrm{M}$ final concentration is recommended. The necessary controls are zero compound concentration, $1 \mathrm{mM}$ colchicine for competition, and $1 \mathrm{mM}$ vinblastine for noncompetitor.

\section{Preparation of buffers}

1. Reconstitute General Tubulin Buffer (BST01-010) with $100 \mathrm{~mL}$ of Milli-Q water and place at $4^{\circ} \mathrm{C}$ for storage.

2. Resuspend GTP vial with $100 \mu \mathrm{L}$ of water, aliquot into $10 \times 10 \mu \mathrm{L}$ and freeze at $70^{\circ} \mathrm{C}$ for storage. 


\section{Preparation of tubulin protein, in 50 assay aliquots}

1. Prepare $1 \mathrm{~mL}$ of ice cold G-PEM buffer: On ice, mix $1 \mathrm{~mL}$ of General Tubulin Buffer with $10 \mu \mathrm{L}$ of GTP stock.

2. Resuspend $500 \mu \mathrm{g}$ of T333 in $60 \mu \mathrm{L}$ of ice cold G-PEM and place back on ice.

3. Microfuge for $5 \mathrm{~min}$ at $14,000 \mathrm{~g}$ at $4{ }^{\circ} \mathrm{C}$.

4. Carefully pipette $55 \mu \mathrm{L}$ of supernatant (avoiding the location where a very small pellet might be located) into a clean tube on ice.

5. Pipette into $10 \times 5 \mu \mathrm{L}$ aliquots on ice, and drop freeze all of them into liquid nitrogen.

6. Store at $-70^{\circ} \mathrm{C}$, where they are stable for 6 months.

\section{Preparation of beads, enough for 50 assays}

This step should be performed just prior to the assays because the change of buffer decreases the shelf life of the beads to $24 \mathrm{~h}$.

1. Pipette $4.4 \mathrm{mg}$ of Streptavidin-SPA beads into a $15 \mathrm{~mL}$ Falcon tube, centrifuge $3000 \mathrm{rpm}$ for $10 \mathrm{~min}$ to pellet the beads.

2. Carefully pipette off the sup, leaving $100 \mu \mathrm{L}$ liquid volume above the beads.

3. Resuspend in $5 \mathrm{~mL}$ of General Tubulin Buffer and centrifuge again.

4. Carefully pipette off the sup, leaving $100 \mu \mathrm{L}$ liquid volume above the beads.

5. Resuspend the beads thoroughly in $9.5 \mathrm{~mL}$ of General Tubulin Buffer.

\section{Preparation of tubulin-beads, enough for 50 assays}

These tubulin loaded beads should be made just prior to assay because they will have a life time of $24 \mathrm{~h}$ at $4^{\circ} \mathrm{C}$.

1. Prepare $1 \mathrm{~mL}$ of ice cold G-PEM buffer: On ice, mix $1 \mathrm{~mL}$ of General Tubulin Buffer with $10 \mu \mathrm{L}$ of GTP stock.

2. Rapidly defrost one vial of biotin tubulin by warming in Milli-Q water for 30 secs, then place on ice.

3. Dilute the vial of biotin tubulin with $500 \mu \mathrm{L}$ of G-PEM buffer.

4. Pipette the entire volume of biotin tubulin into the $9.5 \mathrm{~mL}$ of beads, mix thoroughly incubate on a slow $10 \mathrm{rpm}$ rotator at $4^{\circ} \mathrm{C}$ for $30 \mathrm{~min}$ ), then use immediately, or up to $24 \mathrm{~h}$ thereafter if necessary (activity may drop by $40 \%$ during this time). The tubulin-beads must be kept rotating if aliquots are going to be used continuously over the $24 \mathrm{~h}$ period. 


\section{Preparation of tritium colchicine, enough for 50 assays}

Dilute $50 \mu \mathrm{L}$ of $[3 \mathrm{H}]$ colchicine (specific activity $20 \sim 80 \mathrm{Ci} / \mathrm{mmole}, 1.0 \mu \mathrm{Ci} / \mu \mathrm{L}$, original stock concentration 5 10 $\mu \mathrm{M}$ ) into 500ul of General Tubulin Buffer and mix well. This makes a sub-stock of $0.5 \sim 1.0 \mu \mathrm{M}$ and a final concentration in the reaction of $25 \sim 50 \mathrm{nM}$. If the signal is too low this component can be increased upto fourfold this level, this is particularly useful for non-HTS applications where more signal is required.

\section{Material and equipment required}

Material

Tritiated Colchicine

Streptavidin SPA beads

96-well plates Low PB Corning Costar Inc.

\section{Equipment}

Scintillation Counter Packard Instrument Inc. Tel: 1-800-762-4000 Topcount Microtplate Reader

Scintillation counter plate reader set-up

Measurement type: Top

Sensitivity: Highest.

Read time per well: 10 seconds per read.

Shaking: 5 s medium, orbital.

Plate template: Griener GRE96fb (flat, black). 


\section{Soft Agar Colony Assay Protocol}

\section{Preparation of $1.6 \%$ agar}

1. Pre-warm $50 \mathrm{~mL}$ autoclave-safe glass bottles in a $40^{\circ} \mathrm{C}$ water bath.

2. Heat $1800 \mathrm{~mL}$ of deionized $\mathrm{H}_{2} \mathrm{O}$ in a $4 \mathrm{~L}$ flask with $2 \mathrm{~L}$ calibration to $80 \sim 90^{\circ} \mathrm{C}$ on a hot plate. Use a glass petri cap to prevent evaporation.

3. Add $25 \mathrm{~g}$ of dry Agar to the flask containing the $80 \sim 90^{\circ} \mathrm{C}$ water. Stir with a glass rod until lumps disappear.

4. While the flask is still being heated, adjust the volume to $2000 \mathrm{~mL}$ with H2O. Stir until thoroughly mixed.

5. Add $40 \mathrm{~mL}$ of the $1.6 \%$ agar solution to each of the $100 \mathrm{~mL}$ bottles previously held at $40^{\circ} \mathrm{C}$. Cap bottles loosely.

6. Autoclave for 15 min on slow exhaust. Allow bottles to cool for a few minutes then tighten caps. Store at room temperature or at $4^{\circ} \mathrm{C}$.

\section{Preparation of the $0.5 \%$ agar growth medium dishes}

The directions provided below are for $100 \mathrm{~mL}$ of $0.5 \%$ Agar Growth Medium - enough to prepare about 14 60 $\mathrm{mm}$ dishes. Volumes may be adjusted for larger sized dishes as needed.

1. Prepare the Growth Medium at the desired concentration of FBS, and warm in a $44^{\circ} \mathrm{C}$ water bath.

2. Melt $40 \mathrm{~mL}$ of $1.6 \%$ agar in a microwave oven until just molten, swirling intermittently. Remove the bottle as soon as the agar solution becomes clear.

3. Cool the $1.6 \%$ agar at room temperature for 2 to $3 \mathrm{~min}$.

4. Place the bottle containing the agar in the $37^{\circ} \mathrm{C}$ water bath.

5. When both the Growth Medium and the $1.25 \%$ agar have reached $37^{\circ} \mathrm{C}$, mix the $1.6 \%$ agar with the Growth Medium to make a 0.5\% Agar Growth Medium solution.

6. Pipette $6 \mathrm{~mL}$ of the $0.5 \%$ Agar Growth Medium mixture into each sterile $60 \mathrm{~mm}$ petri dish. Avoid forming bubbles and spread the mixture evenly by slowly rotating the dish. Note: Reagents such as TPA) or EGF should be added to the $0.5 \%$ Agar Growth Medium just before pipetting it into the petri dishes.

Typically, a final concentration of $10 \mathrm{ng} / \mathrm{mL}$ TPA or EGF is used. Control plates to which the solvent (DMSO or water) for the TPA or EGF is added should also be prepared.

7. Allow the $0.5 \%$ Agar Growth Medium layer to harden for 30 to $40 \mathrm{~min}$ at room temperature in a sterile laminar flow hood.

8. Store the dishes overnight in a $37^{\circ} \mathrm{C}$ incubator or seal in a bag and store at $4^{\circ} \mathrm{C}$. The dishes can be stored this way for several days. Note: Refrigerated dishes should be warmed to $37^{\circ} \mathrm{C}$ before use. 


\section{Preparation of cell suspension and performing the over-agar assay}

1. Two or three days before the experiment, plate the cells in a screw-cap T-25 or T75 flask in a final volume of 10 to $15 \mathrm{~mL}$ of Cell Growth Medium.

2. Grow the cells in a $37^{\circ} \mathrm{C}, 5 \% \mathrm{CO}_{2}$ humidified incubator until the cells are 80 to $90 \%$ confluent.

3. Wash the monolayer of cells in T-25 flask or T-75 flask twice with $1 \sim 2 \mathrm{~mL}$ of $\mathrm{Ca}^{2+}, \mathrm{Mg}^{2+}$ free PBS, aspirating the PBS after each wash.

4. Add $1 \mathrm{~mL}$ of $0.03 \%$ Trypsin.

5. Incubate in a $37^{\circ} \mathrm{C}, 5 \% \mathrm{CO}_{2}$ humidified incubator for 3 to $6 \mathrm{~min}$ or until $90 \%$ of the cells are detached.

6. Add $1 \mathrm{~mL}$ of DMEM containing 10\% heat-inactivated FBS (DMEM-FBS).

7. Gently resuspend cells with a sterile pipette three or four times to make a singlecell suspension. Note: Observe a sample of the suspension with a microscope to confirm that it consists of single cells rather than clumps.

8. Determine the concentration of cells and adjust the suspension to $1 \times 10^{3}$ cells $/ \mathrm{mL}$ in DMEM-FBS. Note: If the cells are to be plated on the TPA- or EGF-containing $0.5 \%$ Agar Growth Medium, then TPA or EGF should be added to the cell suspension just before transferring them onto the plates. The final concentration of TPA or EGF should be $10 \mathrm{ng} / \mathrm{mL}$.

9. Transfer $3 \mathrm{~mL}$ of the cell suspension (containing a total of $3 \times 10^{3}$ cells) to the $0.5 \%$ Agar Growth Medium plate and incubate at $37^{\circ} \mathrm{C}, 5 \% \mathrm{CO}_{2}$.

10. Culture the cells on the $0.5 \%$ Agar Growth Medium plate for 14 to 21 days, during which time colonies of transformed cells will form in the TPA- or EGFtreated dishes.

11. Count the number of cells in each colony and the number of colonies using a microscope. Note: Each colony should contain more than eight cells. 


\section{Cell Cycle Analysis Protocol}

1. Human melanoma cells A375 were cultured into $10 \mathrm{~cm}$ tissue culture dishes till $80 \%$ confluence.

2. Serum starved for $24 \mathrm{~h}$. Media was then replaced with media containing testing compounds at different concentration or vector only in triplicates.

3. Cells were incubated with compounds for $6 \mathrm{~h}$, trypsinized, and harvested by centrifugation.

4. Cells were then washed with $1 \times$ PBS 3 times and resuspended in ice-cold $70 \%$ ethanol at $4^{\circ} \mathrm{C}$ overnight.

5. Ethanol was removed by centrifugation and washing with $1 \times \mathrm{PBS} 3$ times.

6. Finally, cells were pelleted out of PBS and resuspended in $1 \mathrm{~mL}$ PBS buffer containing $100 \mu \mathrm{g} / \mathrm{mL}$ RNase A. cells were incubated at $37^{\circ} \mathrm{C}$ for $1 \mathrm{~h}$.

7. After centrifuging cells and aspirating supernatant, resuspend cells in $500 \mu \mathrm{L}$ of propidium iodide $(50 \mu \mathrm{g} / \mathrm{mL})$ in PBS.

8. Cell cycle was determined using a BD LSR-II (BD Biosciences, San Jose, CA USA) with a 10,000 cells scored. Data were analyzed and graphs were prepared using the Modfit 2.0 program (Verity Software House, Topsham, ME). 


\section{Tubulin Imaging Protocol}

\section{Solution preparation (per 96-well plate)}

1. 1X Wash Buffer: Add $30 \mathrm{~mL} 10 \mathrm{X}$ Wash Buffer to $270 \mathrm{~mL}$ ultrapure water. Store buffer at $4{ }^{\circ} \mathrm{C}$ for up to 7 days.

2. Fixation Solution: Add $5 \mathrm{~mL}$ of $16 \%$ formaldehyde solution to $15 \mathrm{~mL}$ of $1 \mathrm{X}$ Wash Buffer and heat to $37^{\circ} \mathrm{C}$ in a water bath just before use. Pre-warming the Fixation Solution is critical for maintaining cell integrity. Prepare solution just before each assay.

3. 1X Permeabilization Buffer: Add $2 \mathrm{~mL}$ of $10 \mathrm{X}$ Permeabilization Buffer to $18 \mathrm{~mL}$ of the 1X Wash Buffer. Store this buffer at $4^{\circ} \mathrm{C}$ for up to 7 days.

4. 1X Blocking Buffer: Add $10 \mathrm{~mL}$ of the $10 \mathrm{X}$ Blocking Buffer to $90 \mathrm{~mL}$ of $1 \mathrm{X}$ Wash Buffer. Store buffer at $4^{\circ} \mathrm{C}$ for up to 7 days.

5. Primary Probe Solution: Reconstitute DY554-phalloidin (F-actin kits) by adding $110 \mu \mathrm{L}$ for 10 units $(550 \mu \mathrm{L}$ for 50 units $)$ of pure methanol and gently mixing. Use the DY554-phalloidin immediately and promptly store any unused portion at $-20^{\circ} \mathrm{C}$.

6. F-actin and tubulin kits $(8402401,8402402)$ : Add $100 \mu \mathrm{L}$ of DY554-phalloidin and $6 \mu \mathrm{L}$ of Tubulin Primary Antibody to $5.9 \mathrm{~mL}$ of $1 \mathrm{X}$ Blocking Buffer. Prepare solution just before each assay. F-actin kits (8402501, 8402502): Add $6 \mu \mathrm{L}$ of DAPI, $100 \mu \mathrm{L}$ of DY554-phalloidin to $5.9 \mathrm{~mL}$ of the $1 \mathrm{X}$ Blocking Buffer. Prepare solution just before each assay.

7. For the tubulin kits $(8402601,8402602)$ : Add $6 \mu \mathrm{L}$ of Tubulin Primary Antibody to $6 \mathrm{~mL}$ of the 1X Blocking Buffer. Prepare solution just before each assay.

8. Staining Solution (Secondary Antibody): F-actin and tubulin kits (8402401, 8402402): Add $6 \mu \mathrm{L}$ of DAPI, $6 \mu \mathrm{L}$ of the DyLight 649 Goat Anti-Mouse to $6 \mathrm{~mL}$ of $1 X$ Blocking Buffer. Prepare solution just before each assay.

9. For the tubulin kits $(8402601,8402602)$ : Add $6 \mu \mathrm{L}$ of DAPI and $6 \mu \mathrm{L}$ of the DyLight 549 Goat Anti-Rabbit to $6 \mathrm{~mL}$ of $1 \mathrm{X}$ Blocking Buffer. Prepare solution just before each assay.

10. Whole Cell Stain Solution: Just before use, reconstitute the Whole Cell Stain Green by adding $50 \mu \mathrm{L}$ of DMSO to the tube and mixing. Add $12 \mu \mathrm{L}$ of the stain in DMSO to $12 \mathrm{~mL}$ of $1 \mathrm{X}$ Wash Buffer. Prepare solution just before each assay. Use the Whole Cell Stain Solution immediately and discard the unused solution. Whole Cell Stain Green in DMSO may be stored at $-20^{\circ} \mathrm{C}$ for $<1$ week.

\section{Protocol for the cytoskeletal rearrangement kit}

1. Dilute the cytochalasin D stock solution (e.g., $3 \mathrm{mM}$ in DMSO) to $30 \mu \mathrm{M}$, or other test compounds to appropriate concentration, in culture medium. Add $50 \mu \mathrm{L}$ of culture medium to the control wells. Add $50 \mu \mathrm{L}$ of cytochalasin D in culture medium to each treatment well $(150 \mu \mathrm{L}$ final $)$. Incubate for $3 \mathrm{~h}$ at $37^{\circ} \mathrm{C}$ in $5 \%$ $\mathrm{CO}_{2}$. 
2. Aspirate culture medium and add $100 \mu \mathrm{L} /$ well of warmed Fixation Solution. Incubate plate in a fume hood at room temperature for $15 \mathrm{~min}$. Using warm $\left(37^{\circ} \mathrm{C}\right)$ Fixation Solution is critical for maintaining cell integrity.

3. Aspirate Fixation Solution and wash plate twice with $100 \mu \mathrm{L} /$ well of $1 \mathrm{X}$ Wash Buffer.

4. Aspirate Wash Buffer, add $100 \mu \mathrm{L} /$ well of $1 \mathrm{X}$ Permeabilization Buffer and incubate for $15 \mathrm{~min}$ at room temperature.

5. Aspirate Permeabilization Buffer. Add $100 \mu \mathrm{L} /$ well of 1X Blocking Buffer. Incubate at room temperature for $15 \mathrm{~min}$.

6. Aspirate Blocking Buffer and add $50 \mu \mathrm{L} /$ well primary probe solutions. Incubate for $1 \mathrm{~h}$ at room temperature. Note: For whole cell stain and F-actin kits, skip step 7 and 8.

7. Aspirate Primary Probe Solution and then wash twice with $100 \mu \mathrm{L} /$ well of $1 \mathrm{X}$ Blocking Buffer.

8. Aspirate Blocking Buffer and add $50 \mu \mathrm{L} /$ well of Staining Solution (secondary antibody). Incubate for $30 \mathrm{~min}$ protected from light at room temperature.

9. Aspirate solution and then wash plate three times with $100 \mu \mathrm{L} /$ well of $1 \mathrm{X}$ Wash Buffer.

10. Add $100 \mu \mathrm{L} /$ well of Whole Cell Stain Solution. Incubate for 15 min protected from light at room temperature.

11. Aspirate Whole Cell Stain Solution and wash plate three times with $100 \mu \mathrm{L} /$ well of 1X Wash Buffer.

12. Aspirate Wash Buffer and replace with $150 \mu \mathrm{L} /$ well of $1 \mathrm{X}$ Wash Buffer.

13. Seal plate and evaluate on the ArrayScan HCS Reader. Store plates at $4^{\circ} \mathrm{C}$. 


\section{VITA}

Zhao Wang was born in Shandong Province, China, in 1975. He joined the Shandong University in 1995 and received a Bachelor of Medicine degree in 2000. He joined the joint Master degree program offered by the Peking Union Medical College (PUMC) and the Chinese National Institute for the Control of Pharmaceutical and Biological Products (NICPBP) in 2000. He received his Master of Pharmaceutical Sciences degree in 2003. After graduation, he worked in NICPBP for two years as former employee with a title of Lab Scientist. In the fall of 2005, he joined the graduate program in the Department of Pharmaceutical Sciences, the University of Tennessee Health Science Center, Memphis, from where he will receive his Ph.D. under the supervision of Prof. Wei Li. 